\title{
Controller Design of Multivariable LTi Unknown Systems
}

by

William Szu-Wei Wang

A thesis submitted in conformity with the requirements for the degree of Master of Applied Science Graduate Department of Electrical and Computer Engineering University of Toronto

Copyright (C) 2011 by William Szu-Wei Wang 


\begin{abstract}
Controller Design of Multivariable LTI Unknown Systems

William Szu-Wei Wang

Master of Applied Science

Graduate Department of Electrical and Computer Engineering

University of Toronto

2011
\end{abstract}

This thesis deals with the design of multivariable controllers for stable linear timeinvariant multi-input multi-output systems, with an unknown mathematical model, subject to constant reference/disturbance signals and actuator saturation constraints. A new controller parameter optimization approach, which can be carried out experimentally with no knowledge of the plant model nor of the order of the system, is proposed. The approach has the advantage that controllers can be optimized by perturbing only the initial conditions of the servocompensator, and that the order of the resulting controller obtained can be specified by the designer. Implementation of the proposed controller design approach is described, and an experimental application study of the proposed method applied to a multivariable system with industrial sensor/actuator components is presented to illustrate the feasibility of the design method in an industrial environment. 


\section{Acknowledgements}

It is my extreme honour and pleasure to have Professor E.J. Davison as my thesis supervisor. My gratitude for his continuous patience, guidance, and insights on "real world" problems is everlasting.

I would also like to extend my appreciation to Bartek Roszak and Simon Lam for their

helping hand in the setup of the MARTS system. Thanks also go to all the members of the Systems Control Group for making my graduate years here an exciting, rewarding, and unforgettable experience that I will forever treasure. 


\section{Table of Contents}

1 Introduction $\quad 1$

1.1 Ziegler-Nichols (for Single-Input Single-Output Systems) . . . . . . . . . 2

1.1.1 Description of Ziegler-Nichols . . . . . . . . . . . . . 2

1.1.2 Extension to Multi-Input and Multi-Output Systems . . . . . . 5

1.2 Extremum Seeking (for Single-Input Single-Output Systems) . . . . . . . 6

1.2.1 Description of Extremum Seeking . . . . . . . . . . . 6

1.2.2 Application of Extremum Seeking for Controller Tuning . . . . . . 7

1.3 Iterative Feedback Tuning (for Multivariable Controllers) . . . . . . . . . 9

1.4 Thesis Outline . . . . . . . . . . . . . . . . . . . . . . 11

2 An Online Optimization Approach to the Robust Servomechanism $\begin{array}{ll}\text { Problem } & 12\end{array}$

2.1 Problem Statement . . . . . . . . . . . . . . . . . . . . 12

2.2 Controller Structure . . . . . . . . . . . . . . . . . . . 14

2.2.1 Motivation ....................... 14

2.2.2 Experimental Calculation of Steady-State Tracking and Disturbance Gain Matrices . . . . . . . . . . . . . . . . . 17

2.3 Tuning Regulator . . . . . . . . . . . . . . . . . . . . . . . 19

2.4 Controller Parameter Selection . . . . . . . . . . . . . . . . . . 23

2.4.1 Optimization Problem A . . . . . . . . . . . . . . 24

2.4.2 Optimization Problem B . . . . . . . . . . . . . . 27

2.5 Proposed Controller Tuning Approach . . . . . . . . . . . . . . . . 30

2.5.1 Tuning for Tracking Performance . . . . . . . . . . . . . . 30

2.5.2 Tuning for Disturbance Rejection Performance . . . . . . . . . . . 30

2.5.3 Using a Non-Noise Sensitive Performance Index . . . . . . . . . . 32

2.6 Controller Comparison . . . . . . . . . . . . . . . . . 33

2.7 Experimental Implementation of Proposed Controller Design . . . . . . . 34 
2.7.1 Performance Index Modification . . . . . . . . . . . . . . 35

2.7 .2 Controller Implementation . . . . . . . . . . . . . . . 36

2.8 Optimization Procedure . . . . . . . . . . . . . . . . . . . . . 37

3 Optimization Algorithm and Simulation Procedure 39

3.1 The Chosen Algorithm . . . . . . . . . . . . . . . . . . . . . 39

3.2 Illustration Example . . . . . . . . . . . . . . . . . . . 43

3.3 Description of the Simulation Procedure . . . . . . . . . . . 46

4 MARTS Simulation Example (Minimum Phase System) 47

4.1 Description of MARTS . . . . . . . . . . . . . . . . . . . 47

4.2 Control Design 1 for MARTS . . . . . . . . . . . . . . . 50

4.3 Control Design 2 for MARTS . . . . . . . . . . . . . 57

4.4 Control Design 1 for MARTS Repeated under the Influence of Noise . . . 64

4.5 Control Design 2 for MARTS Repeated under the Influence of Noise . . . 69

4.6 Control Design 3 for MARTS . . . . . . . . . . . . . . . 76

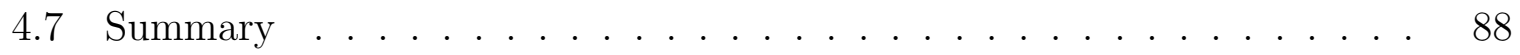

5 Williams and Otto Chemical Plant Example (Minimum Phase System) 89

5.1 Description of the Williams and Otto Chemical Plant . . . . . . . . 89

5.2 Control Design for Williams and Otto Chemical Plant . . . . . . . . . . 95

5.3 Summary . . . . . . . . . . . . . . . . . . . 106

6 Turbo-Generator Example (Nonminimum Phase System) 107

6.1 Description of the Turbo-Generator . . . . . . . . . . . . . . . 107

6.2 Control Design 1 for Turbo-Generator . . . . . . . . . . . . . . . . 110

6.3 Control Design 2 for Turbo-Generator . . . . . . . . . . . . . . . 118

6.4 Summary . . . . . . . . . . . . . . . . . . . . 126

7 Comparison Example $\quad 127$

7.1 Description of the Original Problem . . . . . . . . . . . . . . 127

7.2 Modifications to the Original Problem . . . . . . . . . . . . . 129

7.3 Control Design using Extremum Seeking . . . . . . . . . . . . . 130

7.4 Control Design using Proposed Approach . . . . . . . . . . . . . . 136

7.5 Summary . . . . . . . . . . . . . . . . . . . . . 140 
8 MARTS Experiment $\quad 141$

8.1 Description of MARTS . . . . . . . . . . . . . . . 141

8.2 Developing Linear Control Valve Characteristics . . . . . . . . . . . . . . 144

8.3 Unexpected Observation . . . . . . . . . . . . . . . . . . 147

8.4 Measuring the Steady-State Tracking Matrix . . . . . . . . . . . . . . . . 149

8.5 Control Design for MARTS . . . . . . . . . . . . . . . . . 152

9 Conclusion $\quad 161$

9.1 Summary of Results . . . . . . . . . . . . . . . . . 161

9.2 Future Work . . . . . . . . . . . . . . . . . 162

$\begin{array}{ll}\text { References } & 163\end{array}$

$\begin{array}{ll}\text { A Mathematical Derivations } & 168\end{array}$

A.1 Equivalent Steady-State Control Input Expressions ． . . . . . . . . . . . 168

A.2 Initial Conditions for Optimization Problem B . . . . . . . . . . . . . . . 169

B Model of Williams and Otto Chemical Plant 170

C Detailed Optimization Outputs $\quad 181$

C.1 Control Design for Chemical Plant . . . . . . . . . . . . . . . . 181

C.2 Control Design for Turbo-Generator . . . . . . . . . . . . . . . . . 186

C.3 Control Design for Time-Delayed System . . . . . . . . . . . . . . . . 200

C.4 Control Design for Experimental MARTS . . . . . . . . . . . . . . . 207

$\begin{array}{ll}\text { D Program Listings } & 209\end{array}$

D.1 Valve Linearization . . . . . . . . . . . . . . . . . . . . . . 209

D.2 Proportional Controller Design _ . . . . . . . . . . . . . . 211

D.3 Extremum Seeking . . . . . . . . . . . . . . . . . . . . . . . . . 212

D.4 Optimization Problem A and B . . . . . . . . . . . . . . 216

D.5 MARTS Experiment . . . . . . . . . . . . . 226 


\section{List of Tables}

1.1 PID parameters obtained from Ziegler-Nichols step-response method. . . 3

1.2 PID parameters obtained from Ziegler-Nichols ultimate-sensitivity method. 4

5.1 Summary of controller parameter optimization for the Williams and Otto chemical plant with $\theta=0$ and $\mu=10^{-6}$. . . . . . . . . . . . . 96

5.2 Summary of controller parameter optimization for the Williams and Otto chemical plant with $\theta=20$ and $\mu=10^{-6} \ldots$. . . . . . . . . . . . 96

6.1 Summary of controller parameter optimization $A$ for the Turbo-Generator with $\theta=0$ and $\mu=10^{-4} \ldots \ldots \ldots$. . . . . . . . . . . 111

6.2 Summary of controller parameter optimization $A$ for the Turbo-Generator with $\theta=10$ and $\mu=10^{-4} \ldots \ldots \ldots \ldots$. . . . . . . . . . . 111

6.3 Summary of controller parameter optimization $B$ for the Turbo-Generator with $\theta=0$ and $\mu=10^{-4} \ldots \ldots \ldots \ldots$. . . . . . . . . . . 118

6.4 Summary of controller parameter optimization $B$ for the Turbo-Generator with $\theta=10$ and $\mu=10^{-4} \ldots \ldots \ldots$. . . . . . . . . . . 119

7.1 Summary of controller parameter optimization for the SISO time-delayed system with the cost function spanning from $t_{0}=0 s$ to $T \approx \infty . .$. .

7.2 Summary of controller parameter optimization for the SISO time-delayed system with the cost function spanning from $t_{0}=10 \mathrm{~s}$ to $T \approx \infty \ldots .$.

7.3 Summary of controller parameter optimization for the SISO time-delayed system with the cost function spanning from $t_{0}=18 s$ to $T \approx \infty$. . . . 138

8.1 Summary of controller parameter optimization for the experimental MARTS system with $\theta=0$ and $\mu=1$. 


\section{List of Figures}

1.1 Determination of $R$ and $L$ parameters for the Ziegler-Nichols step re-

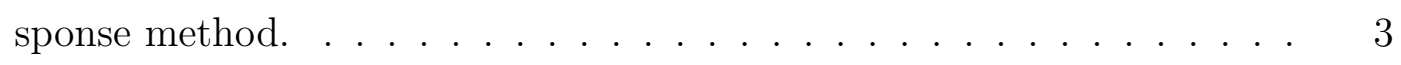

1.2 Multiparameter extremum seeking with $i=1,2, \ldots, m \ldots \ldots \quad 8$

1.3 Overall extremum seeking controller tuning scheme. . . . . . . . . . . 9

2.1 A selection of $\eta_{0}$ values for calculating the average cost. . . . . . . . 35

2.2 The time sequence for the two-term controller. . . . . . . . . . . . . 37

3.1 Possible transformations of the simplex: (a) reflection, (b) expansion, (c) contraction, and $(\mathrm{d})$ shrinkage. . . . . . . . . . . . . . 41

3.2 The sequence of triangles $\left\{T_{k}\right\}$ converging to the optimal point for the Nelder-Mead algorithm. . . . . . . . . . . . . . . . . 41

3.3 Block diagram of the feedback control system. . . . . . . . . . . . . 43

3.4 Root locus of the feedback control system. . . . . . . . . . . . . . . 44

4.1 Schematic of MARTS setup. . . . . . . . . . . . . . . . . 47

4.2 MARTS example, open-loop response. . . . . . . . . . . . . . . . . 49

4.3-1 Control design 1 for the MARTS example, closed-loop response with tuning regulator for the input of $y_{\text {ref }}=\left[\begin{array}{ll}0.2 & 0\end{array}\right]^{\top} \ldots \ldots \ldots \ldots \ldots 1$

4.3-2 Control design 1 for the MARTS example, closed-loop response with tuning regulator for the input of $y_{\text {ref }}=\left[\begin{array}{ll}0 & 0.2\end{array}\right]^{\top} \ldots \ldots \ldots \ldots . \ldots 52$

4.3-3 Control design 1 for the MARTS example, closed-loop response with two-term controller for the input of $y_{r e f}=\left[\begin{array}{ll}0.2 & 0\end{array}\right]^{\top} \ldots \ldots \ldots \ldots$. .

4.3-4 Control design 1 for the MARTS example, closed-loop response with two-term controller for the input of $y_{\text {ref }}=\left[\begin{array}{ll}0 & 0.2\end{array}\right]^{\top} \ldots \ldots \ldots \ldots . . .54$

4.4-1 Control design 2 for the MARTS example, closed-loop response with tuning regulator for the input of $\eta_{0}=\left[\begin{array}{ll}0.2 & 0\end{array}\right]^{\top} \ldots \ldots \ldots \ldots \ldots$ 
4.4-2 Control design 2 for the MARTS example, closed-loop response with tuning regulator for the input of $\eta_{0}=\left[\begin{array}{ll}0 & 0.2\end{array}\right]^{\top} \ldots \ldots \ldots \ldots \ldots$. . . . . 59

4.4-3 Control design 2 for the MARTS example, closed-loop response with two-term controller for the input of $\eta_{0}=\left[\begin{array}{ll}0.2 & 0\end{array}\right]^{\top} \ldots \ldots \ldots \ldots$

4.4-4 Control design 2 for the MARTS example, closed-loop response with two-term controller for the input of $\eta_{0}=\left[\begin{array}{ll}0 & 0.2\end{array}\right]^{\top} \ldots \ldots \ldots \ldots \ldots$. . . . 61

4.5-1 Control design 1 for the MARTS example with noise, closed-loop response with two-term controller for the input of $y_{r e f}=\left[\begin{array}{ll}0.2 & 0\end{array}\right]^{\top} \ldots$.

4.5-2 Control design 1 for the MARTS example with noise, closed-loop response with two-term controller for the input of $y_{\text {ref }}=\left[\begin{array}{ll}0 & 0.2\end{array}\right]^{\top} \ldots \ldots 66$

4.6-1 Control design 2 for the MARTS example with noise, closed-loop response with tuning regulator for the input of $\eta_{0}=\left[\begin{array}{ll}100 & 0\end{array}\right]^{\top} \ldots \ldots .70$

4.6-2 Control design 2 for the MARTS example with noise, closed-loop response with tuning regulator for the input of $\eta_{0}=\left[\begin{array}{ll}0 & 100\end{array}\right]^{\top} \ldots \ldots . .71$

4.6-3 Control design 2 for the MARTS example with noise, closed-loop response with two-term controller for the input of $\eta_{0}=\left[\begin{array}{ll}100 & 0\end{array}\right]^{\top} \ldots \ldots .72$

4.6-4 Control design 2 for the MARTS example with noise, closed-loop response with two-term controller for the input of $\eta_{0}=\left[\begin{array}{ll}0 & 100\end{array}\right]^{\top} \ldots \ldots .73$

4.7-1 Control design 3 for the MARTS example, closed-loop response with tuning regulator for the input of $y_{0}=\left[\begin{array}{ll}-0.1 & 0\end{array}\right]^{\top} \ldots \ldots \ldots \ldots$. . . . 77

4.7-2 Control design 3 for the MARTS example, closed-loop response with tuning regulator for the input of $y_{0}=\left[\begin{array}{ll}0 & -0.15\end{array}\right]^{\top} \ldots \ldots \ldots \ldots 78$

4.7-3 Control design 3 for the MARTS example, closed-loop response with two-term controller for the input of $y_{0}=\left[\begin{array}{ll}-0.1 & 0\end{array}\right]^{\top} \ldots \ldots \ldots \ldots . .79$

4.7-4 Control design 3 for the MARTS example, closed-loop response with two-term controller for the input of $y_{0}=\left[\begin{array}{ll}0 & -0.15\end{array}\right]^{\top} \ldots \ldots \ldots . \quad 80$

4.7-5 Control design 3 for the MARTS example, closed-loop response with two-term controller for the input of $y_{0}=\left[\begin{array}{ll}-0.1 & -0.15\end{array}\right]^{\top} \ldots \ldots \ldots .81$

4.7-6 Control design 3 for the MARTS example, closed-loop response with two-term controller for the input of $y_{r e f}=\left[\begin{array}{ll}0.1 & 0\end{array}\right]^{\top} \ldots \ldots \ldots \ldots$. . .

4.7-7 Control design 3 for the MARTS example, closed-loop response with two-term controller for the input of $y_{\text {ref }}=\left[\begin{array}{ll}0 & 0.15\end{array}\right]^{\top} \ldots \ldots \ldots . . . \quad 83$

4.7-8 Control design 3 for the MARTS example, closed-loop response with two-term controller for the input of $y_{r e f}=\left[\begin{array}{ll}0.1 & 0.15\end{array}\right]^{\top} \ldots \ldots \ldots .$. 
5.1-1 Williams and Otto chemical plant example, open-loop response. . . . . 92

5.1-2 Williams and Otto chemical plant example, open-loop response. . . . . 93

5.1-3 Williams and Otto chemical plant example, open-loop response. . . . . 94

5.1-4 Williams and Otto chemical plant example, open-loop response. . . . . 95

5.2-1 Williams and Otto chemical plant example, closed-loop response with tuning regulator for the input of $\omega=\left[\begin{array}{lll}1 & 0 & 0\end{array}\right]^{\top} \ldots \ldots$. . . . . . . . . . . 97

5.2-2 Williams and Otto chemical plant example, closed-loop response with tuning regulator for the input of $\omega=\left[\begin{array}{lll}0 & 1 & 0\end{array}\right]^{\top} \ldots \ldots \ldots$. . . . . . . . . 98

5.2-3 Williams and Otto chemical plant example, closed-loop response with tuning regulator for the input of $\omega=\left[\begin{array}{lll}0 & 0 & 1\end{array}\right]^{\top} \ldots \ldots$. . . . . . . . . . . 99

5.2-4 Williams and Otto chemical plant example, closed-loop response with two-term controller and $\theta=0$ for the input of $\omega=\left[\begin{array}{lll}1 & 0 & 0\end{array}\right]^{\top}$. . . . . 100

5.2-5 Williams and Otto chemical plant example, closed-loop response with two-term controller and $\theta=20$ for the input of $\omega=\left[\begin{array}{lll}1 & 0 & 0\end{array}\right]^{\top}$. . . . 101

5.2-6 Williams and Otto chemical plant example, closed-loop response with two-term controller and $\theta=0$ for the input of $\omega=\left[\begin{array}{lll}0 & 1 & 0\end{array}\right]^{\top}$. . . . . 102

5.2-7 Williams and Otto chemical plant example, closed-loop response with two-term controller and $\theta=20$ for the input of $\omega=\left[\begin{array}{lll}0 & 1 & 0\end{array}\right]^{\top}$. . . . 103

5.2-8 Williams and Otto chemical plant example, closed-loop response with two-term controller and $\theta=0$ for the input of $\omega=\left[\begin{array}{lll}0 & 0 & 1\end{array}\right]^{\top}$. . . . . 104

5.2-9 Williams and Otto chemical plant example, closed-loop response with two-term controller and $\theta=20$ for the input of $\omega=\left[\begin{array}{lll}0 & 0 & 1\end{array}\right]^{\top}$. . . . 105

6.1 Turbo-generator example, open-loop response. . . . . . . . . . . . . 109

6.2-1 Control design 1 for the Turbo-generator example, closed-loop response with tuning regulator for the input of $y_{r e f}=\left[\begin{array}{ll}1 & 0\end{array}\right]^{\top}$. . . . . . . . . . 112

6.2-2 Control design 1 for the Turbo-generator example, closed-loop response with tuning regulator for the input of $y_{r e f}=\left[\begin{array}{ll}0 & 1\end{array}\right]^{\top} \ldots \ldots$. . . . . . . 113

6.2-3 Control design 1 for the Turbo-generator example, closed-loop response with two-term controller and $\theta=0$ for the input of $y_{\text {ref }}=\left[\begin{array}{ll}1 & 0\end{array}\right]^{\top} \ldots$. . 114

6.2-4 Control design 1 for the Turbo-generator example, closed-loop response with two-term controller and $\theta=10$ for the input of $y_{\text {ref }}=\left[\begin{array}{ll}1 & 0\end{array}\right]^{\top}$. . . 115

6.2-5 Control design 1 for the Turbo-generator example, closed-loop response with two-term controller and $\theta=0$ for the input of $y_{\text {ref }}=\left[\begin{array}{ll}0 & 1\end{array}\right]^{\top} \ldots \ldots$ 
6.2-6 Control design 1 for the Turbo-generator example, closed-loop response with two-term controller and $\theta=10$ for the input of $y_{\text {ref }}=\left[\begin{array}{ll}0 & 1\end{array}\right]^{\top} \ldots 117$

6.3-1 Control design 2 for the Turbo-generator example, closed-loop response with tuning regulator for the input of $\eta_{0}=\left[\begin{array}{ll}1 & 0\end{array}\right]^{\top} \ldots \ldots \ldots \ldots$

6.3-2 Control design 2 for the Turbo-generator example, closed-loop response with tuning regulator for the input of $\eta_{0}=\left[\begin{array}{ll}0 & 1\end{array}\right]^{\top} \ldots \ldots \ldots \ldots . . .2121$

6.3-3 Control design 2 for the Turbo-generator example, closed-loop response with two-term controller and $\theta=0$ for the input of $\eta_{0}=\left[\begin{array}{ll}1 & 0\end{array}\right]^{\top} \ldots \ldots 122$

6.3-4 Control design 2 for the Turbo-generator example, closed-loop response with two-term controller and $\theta=10$ for the input of $\eta_{0}=\left[\begin{array}{ll}1 & 0\end{array}\right]^{\top} \ldots \ldots 123$

6.3-5 Control design 2 for the Turbo-generator example, closed-loop response with two-term controller and $\theta=0$ for the input of $\eta_{0}=\left[\begin{array}{ll}0 & 1\end{array}\right]^{\top} \ldots \ldots 124$

6.3-6 Control design 2 for the Turbo-generator example, closed-loop response with two-term controller and $\theta=10$ for the input of $\eta_{0}=\left[\begin{array}{ll}0 & 1\end{array}\right]^{\top} \ldots \ldots 125$

7.1 Comparison example, open-loop response. . . . . . . . . . . . . . 128

7.2 Comparison example, closed-loop response with PI Controller for the input of $y_{r e f}=1 \ldots \ldots \ldots \ldots \ldots$

7.3-1 Comparison example, evolution of PI parameters. . . . . . . . . . . . . 132

7.3-2 Comparison example, evolution of PI parameters. . . . . . . . . . . . 133

7.3-3 Comparison example, evolution of PI parameters. . . . . . . . . . . . . 134

7.3-4 Comparison example, evolution of PI parameters. . . . . . . . . . . . 135

7.4 Comparison example, closed-loop response with PI Controller for the input of $y_{r e f}=1 \ldots \ldots \ldots \ldots \ldots \ldots$

8.1 Schematic of MARTS setup. . . . . . . . . . . . . . . . . . . . . 142

$8.2 \quad$ MARTS experimental setup. . . . . . . . . . . . . . . . . . . . . . 143

8.3-1 Nonlinear valve characteristics obtained experimentally. . . . . . . . . . 145

8.3-2 Linearized valve characteristics obtained experimentally. . . . . . . . . 146

8.4 A 12 hour record of water level measurements with zero input flow applied to the two vessels. . . . . . . . . . . . . . . . . . 148

8.5-1 MARTS experiment, open-loop response. . . . . . . . . . . . . . 150

8.5-2 MARTS experiment, open-loop response. . . . . . . . . . . . . . . 151

8.6-1 MARTS experiment, closed-loop response with two-term controller for the input of $y_{0}=\left[\begin{array}{ll}-0.1 & 0\end{array}\right]^{\top} \ldots \ldots \ldots \ldots \ldots \ldots \ldots$ 
8.6-2 MARTS experiment, closed-loop response with two-term controller for the input of $y_{0}=\left[\begin{array}{ll}0 & -0.15\end{array}\right]^{\top} \ldots \ldots \ldots \ldots \ldots \ldots \ldots$

8.6-3 MARTS experiment, closed-loop response with two-term controller for the input of $y_{0}=\left[\begin{array}{ll}-0.1 & -0.15\end{array}\right]^{\top} \ldots \ldots \ldots \ldots \ldots \ldots$

8.6-4 MARTS experiment, closed-loop response with two-term controller for the input of $y_{r e f}=\left[\begin{array}{ll}0.1 & 0\end{array}\right]^{\top} \ldots \ldots \ldots \ldots \ldots \ldots$. . . . . . . . . 157

8.6-5 MARTS experiment, closed-loop response with two-term controller for the input of $y_{\text {ref }}=\left[\begin{array}{ll}0 & 0.15\end{array}\right]^{\top} \ldots \ldots \ldots \ldots \ldots \ldots$. . . . . . . . 158

8.6-6 MARTS experiment, closed-loop response with two-term controller for the input of $\omega(1)=$ on, and $\omega(2)=$ off . . . . . . . . . . . . . 159

8.6-7 MARTS experiment, closed-loop response with two-term controller for the input of $\omega(1)=$ off, and $\omega(2)=$ on . . . . . . . . . 160

D.1 Simulink model for extremum seeking controller tuning. . . . . . . . 215

D.2-7 Simulink model for optimization problems A and B. . . . . . . . . 225

D.3-1 Simulink model for MARTS experiment. . . . . . . . . . . . . 232

D.3-2 "Controller" subsystem block. . . . . . . . . . . . . . . . . . 233

D.3-3 "Valve Linearization" subsystem block. . . . . . . . . . . . . . . . . 234

D.3-4 "Spike Filter" subsystem block. . . . . . . . . . . . . . . . . . 235

D.3-5 "Saturation check" subsystem block. . . . . . . . . . . . . . . 235

D.3-6 "Unit Step Response Test" subsystem block. . . . . . . . . . . . . . 235 


\section{Chapter 1}

\section{Introduction}

Many modern control techniques such as Model Predictive Control (MPC), Linear Quadratic Gaussian (LQG) control, and H-infinity control are model-based control methods. If the dynamic model of the process is initially unknown, one can obtain the model by way of system identification. System identification deals with the problem of obtaining a "good" mathematical model of a system, based solely on using the input-output data of a system. The system identification procedure can be broken down into four main steps [1]:

1. Identification tests. This stage of the procedure involves the design of input test signals that would make the measured data informative about system properties.

2. Model order/structure selection. The types of model that are useful for the application at hand depends on a number of factors such as the desired level of model accuracy, the operating conditions of the process, the type of noise in the environment, and any available a priori knowledge about the process structure and dominant time constant.

3. Parameter estimation. Once the experimental data is obtained and model structure is chosen, the parameters of the model can be estimated by using a variety of methods via optimization.

4. Model validation. At this stage, the model property is first compared against the process a priori knowledge. Other methods such as residual analysis and simulations can then be used to check if the model fits well with the test data. 
The above procedure is an iterative process as it is possible for a model to fail the validation stage which may require re-identification. In general, system identification is a time consuming and expensive process, especially in the process industry where processes are typically large scale systems with complex dynamics, e.g., an industrial chemical plant may have a huge number of interacting subsystems. The following quotes from reference [1] suggest some of the downsides of system identification:

"..., after a long step test, an equally long time is needed to perform identification and hence it is often defined as a separate project phase. This is caused by two things. First, many disturbances occur during the long step test. Data slicing is used to cut the bad portion out, which is a tedious process. Secondly, there is no systematic approach to model order selection (or, settling time determination) and model validation/selection. Hence much time is spent using a trial-and-error approach."

"Commonly used methods of model validation are simulation using estimation data or fresh data, whiteness tests residuals and the tests on the independence between the residuals and past MV's ${ }^{1}$. These methods only tell how well the model agrees with the test data. They can neither quantify the model quality with respect to the purpose of closed-loop control, nor can they give good advice for re-identification. The trial-and-error approach in this step makes industrial identification a very expensive practice."

The difficulties in obtaining a process model has motivated the interest and effort made towards developing controller design methods which are non model-based. The Ziegler-Nichols, Extremum Seeking, and Iterative Feedback Tuning are methods that fall under the non model-based controller design category and are reviewed next.

\subsection{Ziegler-Nichols (for Single-Input Single-Output Systems)}

\subsubsection{Description of Ziegler-Nichols}

Ziegler-Nichols (ZN) [2] is a popular heuristic method for tuning the parameters of a Proportional-Integral-Derivative (PID) controller of the form

$$
u(t)=K\left(e(t)+\frac{1}{T_{i}} \int_{0}^{t} e(s) d s+T_{d} \frac{d e(t)}{d t}\right) .
$$

\footnotetext{
${ }^{1} \mathrm{MV}$ stands for Manipulated Variable (or process input)
} 
The method does not require knowledge of the plant's mathematical model and the controller gains can be computed based on a few simple experiments. There are two versions of the ZN tuning rules: the step-response method and the ultimate-sensitivity method [3].

\section{The Step Response Method}

In this method the unit step response of the open-loop system is performed. The tangent to the step response that has the steepest slope of slope $R$ is drawn. The intersection of the tangent with the horizontal coordinate axis gives the apparent deadtime $L$ of the system. See Figure 1.1. The PID controller parameters are then given by Table 1.1 .

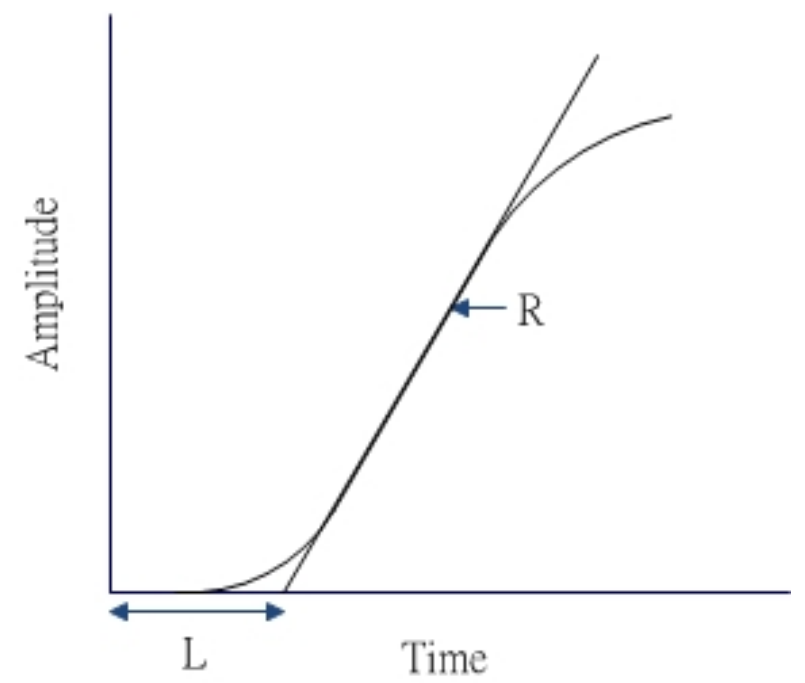

Figure 1.1: Determination of $R$ and $L$ parameters for the Ziegler-Nichols step response method [3].

\begin{tabular}{cccc}
\hline Controller & $K$ & $T_{i}$ & $T_{d}$ \\
\hline $\mathrm{P}$ & $1 /(R L)$ & & \\
$\mathrm{PI}$ & $0.9 /(R L)$ & $3 L$ & \\
$\mathrm{PID}$ & $1.2 /(R L)$ & $2 L$ & $0.5 L$ \\
\hline
\end{tabular}

Table 1.1: PID parameters obtained from Ziegler-Nichols step-response method [3].

The Ultimate-Sensitivity Method

In this method a proportional controller is connected to the plant under a unity 
feedback configuration. The gain of the controller is slowly increased until the closedloop system exhibits an undamped, oscillatory output response. The gain at which this occurs is known as the ultimate gain $\left(K_{u}\right)$ and the period of oscillation is called the ultimate period $\left(T_{u}\right)$. The PID controller parameters are then given by Table 1.2.

\begin{tabular}{cccc}
\hline Controller & $K$ & $T_{i}$ & $T_{d}$ \\
\hline $\mathrm{P}$ & $0.5 K_{u}$ & & \\
$\mathrm{PI}$ & $0.45 K_{u}$ & $T_{u} / 1.2$ & \\
$\mathrm{PID}$ & $0.6 K_{u}$ & $T_{u} / 2$ & $T_{u} / 8$ \\
\hline
\end{tabular}

Table 1.2: PID parameters obtained from Ziegler-Nichols ultimate-sensitivity method [3].

Even though the ZN method is very straight forward to use and often produces acceptable closed-loop performance, there are still a number of disadvantages listed as follows.

- The method is applicable only to single-input single-output (SISO) systems that are open-loop asymptotically stable.

- The method is designed for noise-free conditions and does not take into account actuator saturation constraints.

- The tuning rules do not apply to other controller structures such as integral or proportional-derivative control.

- The ultimate-sensitivity method of the ZN tuning rules requires the system to be brought to its stability limits, which could cause the system to become out of control.

- The tuning rules were originally designed for disturbance rejection performance and may give poor tracking performance.

- The step response method of the ZN tuning rules assumes that the system can be described as a first order time delay process of the form

$$
G(s)=\frac{K e^{-s T}}{(a+s)} .
$$


For systems with a low time delay/time constant ratio the $R$ and $L$ parameters extracted from the unit step response will have small values. This will cause both the proportional and integral gains to become very large [4].

There are a number of methods that can be found in the literature that are similar to the ZN method. The Kappa-Tau tuning rules [5] characterizes the process dynamics using three parameters instead of two to give better closed-loop performance. The phase and amplitude margins design method [6] provides an alternative way of finding the ultimate gain and ultimate period without pushing the system to its stability limits. A description of other single-loop PID tuning techniques can be found in a survey presented by Åström et al. [7].

\subsubsection{Extension to Multi-Input and Multi-Output Systems}

One possible approach for extending the application of the ZN method to multi-input and multi-output (MIMO) asymptotically stable systems is by using a multi-loop PID scheme, where PID controllers are tuned specifically for each pair of inputs and outputs via the ZN method. The one-to-one pairing of the inputs and outputs can be selected based on a matrix known as the Relative Gain Array (RGA) [8]. RGA provides an indication of the level of interaction which occurs between input-output pairs and can be obtained experimentally as follows. Assume that we have a two input and two output system

$$
\left[\begin{array}{l}
y_{1} \\
y_{2}
\end{array}\right]=\left[\begin{array}{ll}
g_{1}(s) & g_{2}(s) \\
g_{3}(s) & g_{4}(s)
\end{array}\right]\left[\begin{array}{l}
u_{1} \\
u_{2}
\end{array}\right]
$$

and that we wish to control the system using the decentralized controller

$$
\begin{aligned}
& u_{1}=K_{1}(s) y_{1} \\
& u_{2}=K_{2}(s) y_{2} .
\end{aligned}
$$

Then to measure the level of interaction existing between these two controllers, we perform the following two sets of experiments:

For $u_{1}=K_{1}(s) y_{1}$ :

First set $u_{2}=0$ and measure the process gain:

$$
u_{2}=0: \quad \text { process gain } G_{1}=\frac{y_{1}(\infty)}{u_{1}(\infty)}=g_{1}(0)
$$


Next, regulate output $y_{2}$ to be 0 and measure the process gain:

$$
y_{2}=0: \quad \text { process gain } G_{2}=\frac{y_{1}(\infty)}{u_{1}(\infty)}=g_{1}(0)-\frac{g_{3}(0) g_{2}(0)}{g_{4}(0)}
$$

The corresponding RGA measure for controller (1.4) is

$$
\lambda_{11}=\frac{G_{1}}{G_{2}} .
$$

For $u_{2}=K_{2}(s) y_{2}$ :

First set $u_{1}=0$ and measure the process gain:

$$
u_{1}=0: \quad \text { process gain } G_{3}=\frac{y_{2}(\infty)}{u_{2}(\infty)}=g_{4}(0) .
$$

Next, regulate output $y_{1}$ to be 0 and measure the process gain:

$$
y_{1}=0: \quad \text { process gain } G_{4}=\frac{y_{2}(\infty)}{u_{2}(\infty)}=g_{4}(0)-\frac{g_{3}(0) g_{2}(0)}{g_{1}(0)} \text {. }
$$

The corresponding RGA measure for controller (1.5) is

$$
\lambda_{22}=\frac{G_{3}}{G_{4}} .
$$

According to Bristol [8], if $\lambda_{i i}$ is positive and has a value of 1 , this means that the control of output $y_{i}$ using input $u_{i}$ is unaffected by other control loops for $i=1,2$. Values that are much larger than 1 implies that independent control of each output is difficult to achieve, and input-output pairs that give rise to negative $\lambda$ values should be avoided as the system can become unstable. It is to be noted that counter-examples can be given to these "rules" [9].

\subsection{Extremum Seeking (for Single-Input Single-Ou- tput Systems)}

\subsubsection{Description of Extremum Seeking}

Extremum seeking (ES) [10] is a nonmodel-based method that can be used either for seeking an unknown set point to achieve an optimal value of an unknown output function, or for tuning parameters of a feedback law. In general, it is mainly applied to SISO systems. Figure 1.2 shows a block diagram of the general sinusoidal perturbation-based 
extremum seeking scheme. To illustrate how ES works, consider the search for only one unknown parameter initially. As described in [10], the input $\theta$ of an output objective function $f(\theta)$ is perturbed by a "slow" sinusoidal signal thereby creating a periodic response at the output. The high pass filter extracts the variation of the objective function in response to the perturbation signal or dither signal. The gradient $\nabla f$ is estimated by demodulating the filtered signal with a sinusoid of the same frequency as the dither signal. The gradient estimate is then integrated, yielding a new parameter estimate $\hat{\theta}$, which converges to the optimal parameters $\theta^{*}$. A channel is composed of a high pass filter, a demodulating signal, an integrator and a dither signal. If there are now multiple unknown parameters to tune, a matching number of channels will be required, each with a different dither frequency since all channels share the same output $f(\theta)$. The overall feedback system operates on three different time scales [11]:

- fastest - the plant with the stabilizing controller,

- medium - the periodic perturbation,

- slowest - the filters in the extremum seeking scheme.

If the perturbation signal is slow, then the plant can be treated as a static map and its dynamics do not interfere with the extremum seeking scheme. In general, the plant is assumed to be open-loop asymptotically stable.

\subsubsection{Application of Extremum Seeking for Controller Tuning}

The extremum seeking scheme described in Section 1.2.1 can be applied to tune parameters of a controller using the setup shown in Figure 1.3. The step-response experiment, which is contained within the upper dash box in Figure 1.3, is carried out iteratively. The cost $J(\theta(k))$ is calculated during the step-response experiment with the controller parameters set as $\theta(k)$ at iteration $k$. The discrete version of the ES algorithm uses the value $J(\theta(k))$ to generate the next set of controller parameters $\theta(k+1)$ in the algorithm. Another step response experiment is then performed with the new parameters, and the process continues iteratively until the optimal parameters are found. This optimization approach has the advantage that no knowledge of the mathematical model of the plant is required. The time scale separation between the plant and the ES optimizer can be achieved by activating the ES optimizer only at instances when the step-response experiment reaches steady state. This is equivalent to decelerating the dynamics of the 


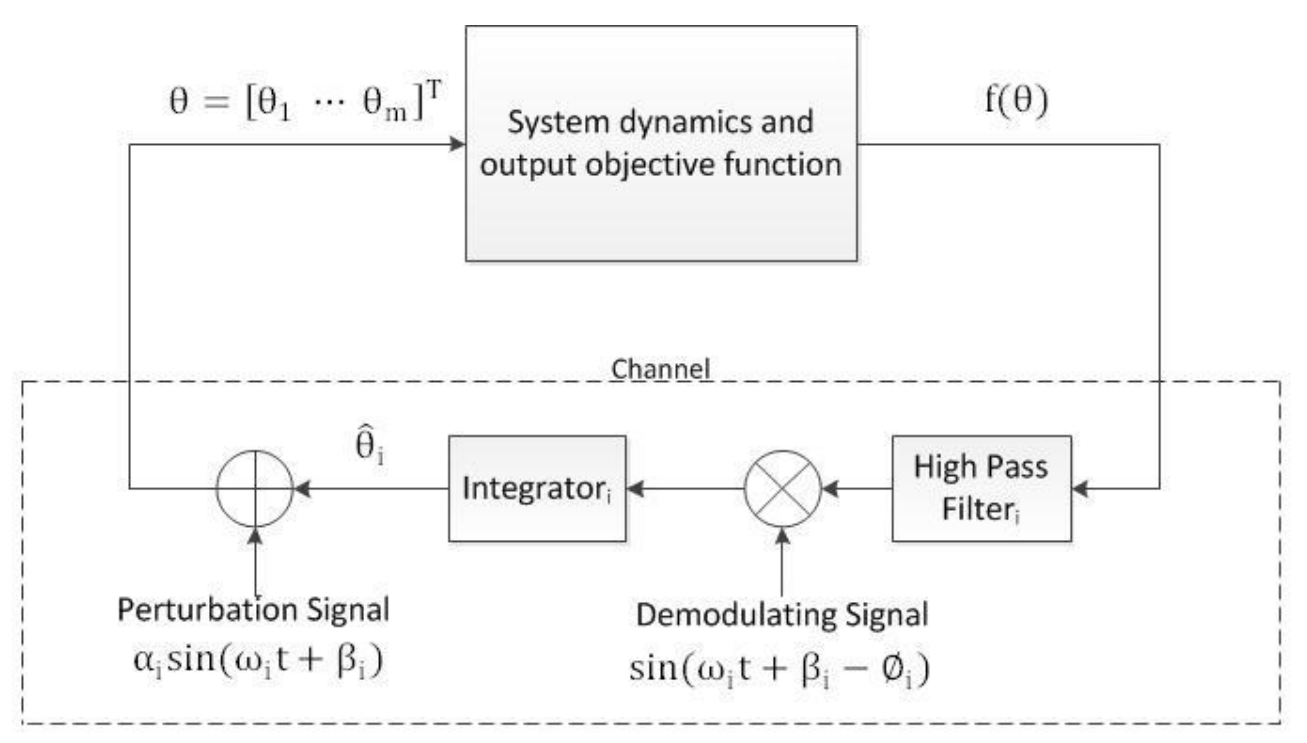

Figure 1.2: Multiparameter extremum seeking with $i=1,2, \ldots, m$. Each unknown parameter of interest $\theta_{i}$ requires 1 channel for tuning [10].

optimizer [12]. According to [13], the discrete ES algorithm can be implemented using the following equations, which are equivalent to the system representation shown in the lower dash box in Figure 1.3,

$$
\begin{aligned}
\zeta(k) & =-h \zeta(k-1)+J(\theta(k-1)) \\
\hat{\theta}_{i}(k+1) & =\hat{\theta}_{i}(k)-\gamma_{i} \alpha_{i} \cos \left(\omega_{i} k\right)[J(\theta(k))-(1+h) \zeta(k)] \\
\theta_{i}(k+1) & =\hat{\theta}_{i}(k+1)+\alpha_{i} \cos \left(\omega_{i}(k+1)\right)
\end{aligned}
$$

where the subscript $i$ indicates the $i^{t h}$ entry of a vector, $\zeta(k)$ is a scalar, $\gamma_{i}$ is the adaptation gain, $\alpha_{i}$ is the perturbation amplitude, and $\omega_{i}$ is the dither frequency. $\omega_{i}$ is chosen such that $\omega_{i}=a^{i} \pi$ with $0<a<1$. Additionally, the high pass filter is designed with $0<h<1$ and a cutoff frequency well below $\omega_{i}$.

Setting up an extremum seeking controller tuning scheme for an unknown system is in general a non-trivial task since the achievable domain of attraction and convergence rate of the ES scheme depends on careful selections of $\gamma, \alpha, \omega, h$, and the initial conditions of $\zeta(0)$. The convergence speed of the overall system is affected by the magnitude of $\gamma \alpha^{2}$. The larger $\gamma \alpha^{2}$ is, the faster the convergence becomes, but now the domain of attraction becomes smaller [14]. Large $\gamma$ and $\omega$ speeds up the convergence rate, but causes the closed-loop system to be sensitive to noise and can lead to steady state tracking error between the estimated optimal controller parameters $\hat{\theta}$ and the true optimal parameters 


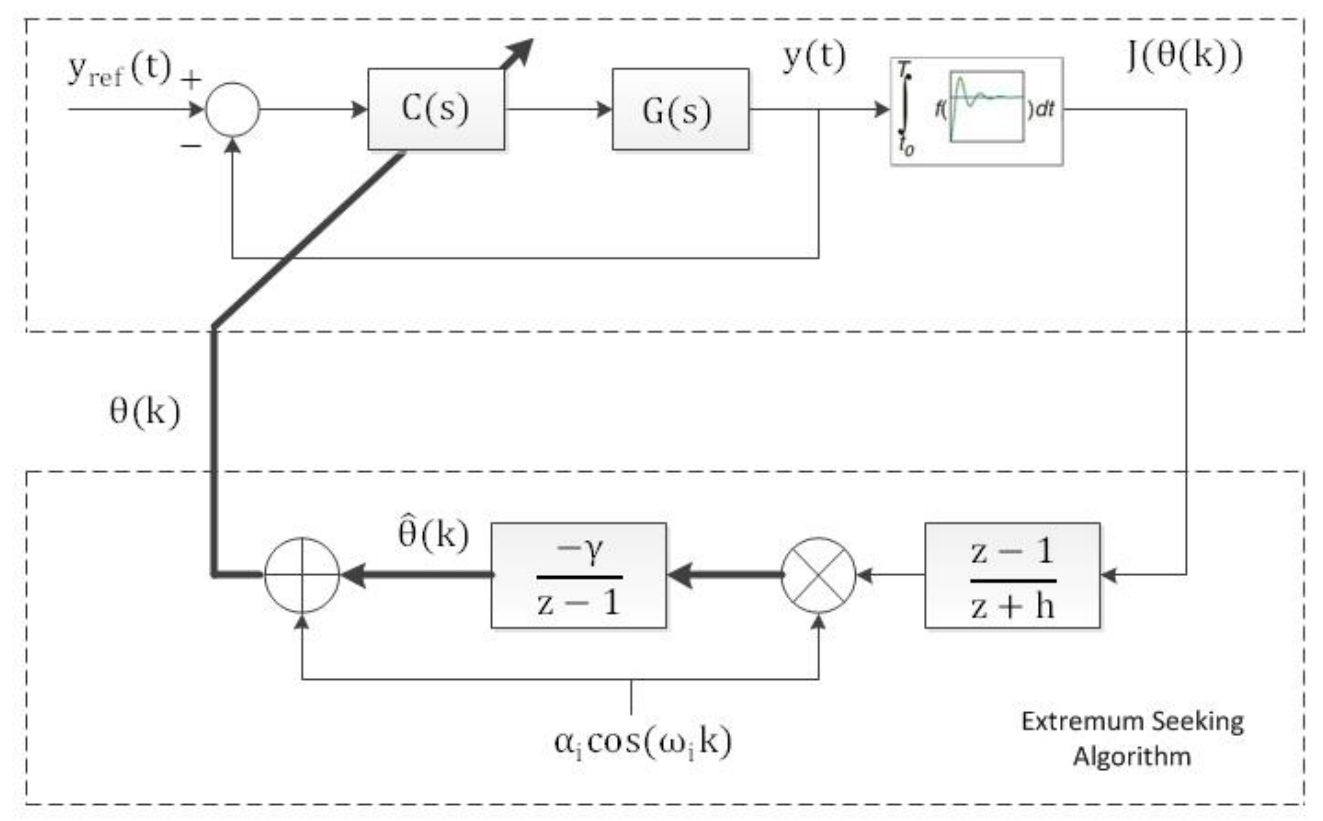

Figure 1.3: Overall extremum seeking controller tuning scheme [13].

$\theta^{*}[15]$. The perturbation or dither amplitude $\alpha$ should be chosen sufficiently large to overcome the noise effect, and at the same time sufficiently small to obtain convergence to a smaller neighborhood of the optimal values and to prevent the closed-loop system from becoming unstable [16]. By examining equations 1.12 to 1.14 it appears that the choice of the initial condition $\zeta(0)$ also has an impact on the convergence rate and stability of the system. The extremum seeking scheme shown in Figure 1.2 assumes that the design of $m$ channels for the identification/tracking of $m$ parameters is required. As stated in [10], there are however no systematic means of multivariable design for unknown systems, using this approach.

\subsection{Iterative Feedback Tuning (for Multivariable C- ontrollers)}

Another gradient-based, model-free tuning method is the iterative feedback tuning (IFT) [17]. In the IFT scheme the following cost function is adopted:

$$
J(\rho)=\frac{1}{2 N} E\left[\sum_{k=1}^{N}\left(L_{y} \tilde{y}_{k}(\rho)\right)^{2}+\lambda \sum_{k=1}^{N}\left(L_{u} u_{k}(\rho)\right)^{2}\right]
$$


where $\rho$ is a vector that contains the controller parameters, $L_{y}$ and $L_{u}$ are frequency weighting filters, $\tilde{y}_{k}$ denotes the error between the achieved and desired output response, $u_{k}$ is the control input, and the subscript $k$ represents discrete time instants. IFT attempts to minimize the cost function $J(\rho)$ by iteratively estimating the gradient of the cost function $\frac{\partial J}{\partial \rho}\left(\rho_{i}\right)$ with respect to the current controller parameters $\rho_{i}$.

At each step of the iterative design for a SISO plant, IFT uses only input-output data from three closed-loop experiments to estimate the gradient of the cost function. The first and third experiments involve a standard servomechanism setup where a reference signal is given and the input-output data is collected. The second experiment is different. It involves subtracting the output signal from the first experiment from the reference, and using this error signal as the new reference signal. The controller parameters are then updated by using a stochastic approximation algorithm of the form

$$
\rho_{i+1}=\rho_{i}-\gamma_{i} R_{i}^{-1} \frac{\partial J}{\partial \rho}\left(\rho_{i}\right)
$$

where $R_{i}$ is the Hessian of the cost function $J$ and $\gamma_{i}$ is the step size. Estimations of $R_{i}$ and $\frac{\partial J}{\partial \rho}\left(\rho_{i}\right)$ can be computed using the data obtained from the three experiments.

One of the drawbacks of IFT, as discussed in [17], is that when feeding back the output at the reference input which is measured during the first experiment, noise that was captured with the output signal will be reinjected into the system during the second experiment and may cause unacceptable system behavior to occur. Another disadvantage of IFT is the increase in complexity when applying to MIMO systems. It is shown in [18] that if a plant has $r$ outputs and $m$ control inputs, $r \times m$ additional experiments are required to be able to compute an estimate of the gradient.

To overcome the disadvantages of the various tuning methods discussed, in this thesis we propose an alternative solution to the design of practical controllers for process control type problems. The proposed approach provides a systematic method of multivariable design for MIMO unknown systems, copes with stability and actuator saturation constraints, and requires essentially no a priori knowledge of the plant. In process industries, the plants or processes are generally assumed to be open-loop asymptotically stable and subject to slow time-varying reference inputs and unmeasurable disturbances that can be approximated as constant signals [19]. The material presented in this thesis is developed within the discrete time framework, as computer-based automation systems are often used within the process industries. 


\subsection{Thesis Outline}

In Chapter 2, the properties of a tuning regulator and a multivariable two-term controller that solve the robust servomechanism problem for unknown multi-input and multi-output systems are first discussed, and then an online controller parameter optimization method for designing a two-term controller is proposed. The focus of Chapter 2 then shifts to a discussion on the implementation of the proposed controller design approach, and in Chapter 3, the mechanism and properties of a chosen optimization algorithm that is used to solve the optimization problem is presented. The proposed design method is then illustrated through a number of simulation examples: a small-scale minimum phase system (Chapter 4), a large-scale minimum phase system (Chapter 5), a mediumscale nonminimum phase system (Chapter 6), and a single-input and single-output time delayed system (Chapter 7). Finally, an experimental application study of the proposed controller design approach is presented in Chapter 8. 


\section{Chapter 2}

\section{An Online Optimization Approach to the Robust Servomechanism Problem}

A method for designing multivariable controllers that solves the robust servomechanism problem for mathematically unknown systems is proposed. The method involves performing real-time online parameter optimization on the system subject to stability and saturation constraints.

\subsection{Problem Statement}

Assume a sampled system to be controlled, described by the following linear shiftinvariant model:

$$
\begin{aligned}
x_{k+1} & =A x_{k}+B u_{k}+E \omega \\
y_{k} & =C x_{k}+F \omega \\
e_{k} & =y_{k}-y_{r e f}
\end{aligned}
$$

where $x_{k} \in \mathbb{R}^{n}$ is the state of the system, $u_{k} \in \mathbb{R}^{m}$ is the input, $y_{k} \in \mathbb{R}^{r}$ is the output, $\omega \in \mathbb{R}^{\Omega}$ is a constant input disturbance, $y_{\text {ref }} \in \mathbb{R}^{r}$ is a constant reference input, $e_{k} \in \mathbb{R}^{r}$ is the error in the system, and there is no knowledge of the plant parameters $(A, B, C, E, F)$ nor of the order $n$. It is assumed, however, that $A$ is asymptotically stable ${ }^{1}$, the control

\footnotetext{
${ }^{1}$ i.e., the eigenvalues of $A$ are all contained in the unit disk centered about the origin of the complex plane.
} 
input $u_{k}$ can be excited, the outputs $y_{k}$ can be measured, and that the plant is sampled with a sampling period $h>0$. The objective is to find a controller to solve the robust servomechanism problem (RSP), as described in [20], for this system, i.e., to find a linear, shift-invariant controller so that

1. the closed-loop system is asymptotically stable,

2. $\lim _{k \rightarrow \infty} e_{k}=0$ for all $x_{0} \in \mathbb{R}^{n}, u_{0} \in \mathbb{R}^{m}, \omega \in \mathbb{R}^{\Omega}, y_{r e f} \in \mathbb{R}^{r}$, and

3. property 2 holds for all perturbations in the plant model which do not cause the perturbed closed-loop system to become unstable.

Lemma 2.1 ([20]). The necessary and sufficient conditions for the existence of a solution to the RSP are that the following conditions should all hold:

1. $(C, A, B)$ is stabilizable and detectable,

2. $m \geq r$, and

3. $(C, A, B)$ has no transmission zeros at 1, i.e., $\operatorname{rank}\left(\left[\begin{array}{cc}A-I & B \\ C & 0\end{array}\right]\right)=n+r$.

Definition 2.1. Define the steady-state tracking gain matrix of the system (2.1) to be

$$
\Gamma=C(I-A)^{-1} B
$$

It is shown in [21] that $\Gamma$ can be measured experimentally by carrying out a finite number of steady-state experiments on the system.

Since the system has been assumed to be open-loop asymptotically stable, the conditions of Lemma 2.1 are equivalent to requiring the steady-state tracking matrix $\Gamma$ to have full row rank [21], i.e., $\operatorname{rank}(\Gamma)=r$. It has been shown in [22] that if $\operatorname{rank}(\Gamma)<r$, then there exists constant $\omega$ and $y_{\text {ref }}$ signals so that for every control law $u_{k}$, either $x_{k}$ will be unbounded or error regulation will not occur.

In reality, actuator saturation constraints are always present in every "real world" problem; thus, an additional necessary condition for the existence of a solution to RSP is that the control inputs of the plant should be sized so that the steady-state value of the $i^{\text {th }}$ control input $u_{\infty}(i)$ is within the saturation bounds of the $i^{\text {th }}$ actuator $u_{\text {Sat }}(i)$, $i=1,2, \ldots, m$; otherwise, error regulation cannot be achieved [23]. In particular, it is assumed that the tracking and disturbance signals applied to the plant have the property that $\left|u_{\infty}(i)\right| \leq\left|u_{S a t}(i)\right|, i=1,2, \ldots, m$. 


\subsection{Controller Structure}

Assuming that $\operatorname{rank}(\Gamma)=r$, then the following robust servomechanism controller for the plant (2.1) is now considered [22]

$$
u_{k}=K_{0} \hat{x_{k}}+K_{1} \eta_{k}
$$

where $\eta_{k}$ is the output of a servocompensator, and where $\hat{x_{k}}$ is the output of a stabilizing compensator. For the case of a constant disturbance and tracking signal, the servocompensator is a controller defined as

$$
\eta_{k+1}=\eta_{k}+h e_{k}
$$

with input $e_{k}$, output $\eta_{k}$, and $h$ is the sampling period. A stabilizing compensator has the following general structure:

$$
\begin{aligned}
\xi_{k+1} & =\Lambda_{0} \xi_{k}+\Lambda_{1} y_{k}+\Lambda_{2} \eta_{k}+\Lambda_{3} y_{r e f} \\
\hat{x_{k}} & =\Lambda_{5} \xi_{k}+\Lambda_{6} y_{k}+\Lambda_{7} y_{r e f}
\end{aligned}
$$

with inputs $y_{k}, \eta_{k}, y_{r e f}, u_{k}$ and output $\hat{x_{k}}$. As an example of a controller which satisfies (2.3) and (2.4), assume that $m=r$ and consider the multivariable two-term controller of the form

$$
u_{k}=-S\left(K_{P} y_{k}+K_{I} \eta_{k}\right)
$$

where $S=\Gamma^{-1}$ is a decoupling matrix, and $K_{P}, K_{I}$ are scalar parameters of the controller. It is to be noted that if $m>r$, we replace the inverse of the $\Gamma$ matrix by its pseudoinverse, i.e., $S=\Gamma^{+}$where $(\cdot)^{+}=(\cdot)^{\top}\left[(\cdot)(\cdot)^{\top}\right]^{-1}$.

\subsubsection{Motivation}

To give motivation for choosing controller (2.6), we will use the results of the following lemma. The proof of this lemma follows the approach taken in [24], which assumes a sample time of $h=1$.

Lemma 2.2. Consider the system

$$
\begin{aligned}
x_{k+1} & =A x_{k}+B u_{k} \\
y_{k} & =C x_{k}
\end{aligned}
$$

subject to the following assumptions: 
1. $A$ is asymptotically stable,

2. $\Gamma^{-1}$ exists, and

3. $K$ is chosen so that $\Gamma K$ has eigenvalues contained in the open left complex plane, e.g., $K=-\epsilon \Gamma^{-1}$ where $0<\epsilon<1$,

and let $Q \in \mathbb{R}^{m \times n}$ be a constant matrix; then there exists $\theta^{*}>0$ such that, for all $\theta \in\left(0, \theta^{*}\right]$

$$
\mathcal{A} \triangleq\left[\begin{array}{cc}
A+\theta B Q & \theta B K \\
h C & I
\end{array}\right]
$$

is asymptotically stable.

Proof. Let $\Phi \triangleq\left[C(I-A)^{-1} B\right] K$. There exists an $\epsilon^{*}>0$ such that, for all $\theta \in\left(0, \epsilon^{*}\right]$, $I+\theta h \Phi$ has eigenvalues within the unit disk and is asymptotically stable. Since $A$ and $I+\theta h \Phi$ are asymptotically stable, there exists unique positive definite matrices $M, N$ that satisfy the discrete Lyapunov equations

$$
\begin{aligned}
A^{\top} M A-M & =-I \\
(I+\theta h \Phi)^{\top} N(I+\theta h \Phi)-N & =-I .
\end{aligned}
$$

Apply the coordinate transformation $\mathcal{A} \mapsto T^{-1} \mathcal{A} T$ :

$$
\begin{aligned}
\Psi & =T^{-1} \mathcal{A} T \\
& =\left[\begin{array}{cc}
I & -(I-A)^{-1} B K \theta \\
0 & \theta I
\end{array}\right]\left[\begin{array}{cc}
A+\theta B Q & \theta B K \\
h C & I
\end{array}\right]\left[\begin{array}{cc}
I & (I-A)^{-1} B K \\
0 & \frac{I}{\theta}
\end{array}\right] \\
& =\left[\begin{array}{cc}
A+\theta \Delta_{1}(\theta) & \theta \Delta_{3}(\theta) \\
\theta \Delta_{2}(\theta) & I+\theta \Delta_{4}(\theta)
\end{array}\right]
\end{aligned}
$$

where

$$
\begin{aligned}
& \Delta_{1}(\theta)=B Q-h(I-A)^{-1} B K C \\
& \Delta_{2}(\theta)=h C \\
& \Delta_{3}(\theta)=-h(I-A)^{-1} B K \Phi+B Q(I-A)^{-1} B K \\
& \Delta_{4}(\theta)=h \Phi .
\end{aligned}
$$

Since eigenvalues are invariant under coordinate transformations, $\mathcal{A}$ is stable if and only if $\Psi$ stable. Consider the system $\xi_{k+1}=\Psi \xi_{k}$ and define a tentative positive definite 
Lyapunov function $V\left(\xi_{k}\right)$ :

$$
V\left(\xi_{k}\right)=\xi_{k}^{\top}\left[\begin{array}{cc}
M & 0 \\
0 & N
\end{array}\right] \xi_{k}
$$

where $M>0$ and $N>0$, then it remains to show that $\Delta V(\xi) \triangleq V\left(\xi_{k+1}\right)-V\left(\xi_{k}\right)$ is negative definite for $\Psi$ to be asymptotically stable.

$$
\begin{aligned}
\Delta V(\xi) & =\xi_{k}^{\top}\left(\Psi^{\top}\left[\begin{array}{cc}
M & 0 \\
0 & N
\end{array}\right] \Psi-\left[\begin{array}{cc}
M & 0 \\
0 & N
\end{array}\right]\right) \xi_{k} \\
& \triangleq \xi_{k}^{\top} \tilde{\mathcal{S}} \xi_{k}
\end{aligned}
$$

where

$$
\tilde{\mathcal{S}}=\left[\begin{array}{c|c}
A^{\top} M A-M & \\
+\theta\left(A^{\top} M \Delta_{1}+\Delta_{1}^{\top} M A\right) & \theta\left(A^{\top}+\theta \Delta_{1}^{\top}\right) M \Delta_{3} \\
+\theta^{2}\left(\Delta_{1}^{\top} M \Delta_{1}+\Delta_{2}^{\top} N \Delta_{2}\right) & +\theta \Delta_{2}^{\top} N\left(I+\theta \Delta_{4}\right) \\
\hline \theta \Delta_{3}^{\top} M\left(A+\theta \Delta_{1}\right) & \left(I+\theta \Delta_{4}\right)^{\top} N\left(I+\theta \Delta_{4}\right)-N \\
+\theta\left(I+\theta \Delta_{4}{ }^{\top}\right) N \Delta_{2} & +\theta^{3}\left(\Delta_{4}^{\top} N \Delta_{5}+\Delta_{5}^{\top} N \Delta_{4}\right) \\
& +\theta^{2}\left(N \Delta_{5}+\Delta_{5}^{\top} N\right)+\theta^{2} \Delta_{3}^{\top} M \Delta_{3}
\end{array}\right] .
$$

Since $\tilde{\mathcal{S}}$ is a symmetric matrix, the principal minors can be used to determine if $\tilde{\mathcal{S}}$ is negative definite. Notice that for sufficiently small values of $\theta$ the main diagonal elements of $\tilde{\mathcal{S}}$ dominate. From (2.9) it is found that the diagonal elements reduce to $-I$ as $\theta \rightarrow 0$; thus, by inspection, there exists a $\theta^{*}>0$ such that, for all $\theta \in\left(0, \theta^{*}\right], \tilde{\mathcal{S}}<0$, which implies $\Delta V(\xi \neq 0)<0$.

On applying the controller (2.6) to the plant (2.1) we obtain the following closed-loop augmented system:

$$
\left[\begin{array}{c}
x_{k+1} \\
\eta_{k+1}
\end{array}\right]=\left[\begin{array}{cc}
A-B S K_{P} C & -B S K_{I} \\
h C & I
\end{array}\right]\left[\begin{array}{l}
x_{k} \\
\eta_{k}
\end{array}\right]+\left[\begin{array}{cc}
E-B S K_{P} F & 0 \\
h F & -h
\end{array}\right]\left[\begin{array}{c}
\omega \\
y_{r e f}
\end{array}\right] .
$$

Since $A$ is stable, there exists a $K_{P}{ }^{*}>0$ so that $A-B S K_{P} C$ is stable for all $K_{P} \in$ $\left[0, K_{P}{ }^{*}\right]$. A stable state matrix $A-B S K_{P} C$ gives rise to a well defined steady-state tracking gain matrix $C\left(I-\left(A-B S K_{P} C\right)\right)^{-1} B$. Recall that $C(I-A)^{-1} B$ has full rank from the assumption that there exists a solution to the servomechanism problem, then by continuity, $\left(C\left(I-\left(A-B S K_{P} C\right)\right)^{-1} B\right)^{-1}$ exists. Since (see Appendix A.1)

$$
\left(C\left(I-\left(A-B S K_{P} C\right)\right)^{-1} B\right)^{-1}=\left(C(I-A)^{-1} B\right)^{-1}+S K_{P},
$$


then

$$
-\Gamma\left(C\left(I-\left(A-B S K_{P} C\right)\right)^{-1} B\right)^{-1}=-\left(1+K_{P}\right) I
$$

has its eigenvalues contained in the open left complex plane, which implies that its inverse $-\left(C\left(I-\left(A-B S K_{P} C\right)\right)^{-1} B\right) S$ also has eigenvalues in the open left complex plane. Thus from Lemma 2.2, for a given $K_{P} \in\left[0, K_{P}{ }^{*}\right]$, there exists a $K_{I}{ }^{*}>0$ such that, for all $K_{I} \in\left(0, K_{I}^{*}\right]$,

$$
\left[\begin{array}{cc}
A-B S K_{P} C & -B S K_{I} \\
h C & I
\end{array}\right]
$$

is asymptotically stable. In other words, sufficiently small $K_{P}$ and $K_{I}$ parameters of controller (2.6) guarantee closed-loop stability of the augmented plant (2.13), and it is shown in [25] that any compensator that stabilizes the augmented plant solves the RSP. Furthermore, the controller has a parameter space that is independent of the system order, and it can be applied to plants without a knowledge of their mathematical models, since the $S$ matrix (2.6) can be determined experimentally.

\subsubsection{Experimental Calculation of Steady-State Tracking and Disturbance Gain Matrices}

Recall system (2.1)

$$
\begin{aligned}
x_{k+1} & =A x_{k}+B u_{k}+E w \\
y_{k} & =C x_{k}+F w \\
e_{k} & =y_{k}-y_{r e f}
\end{aligned}
$$

where $x_{k} \in \mathbb{R}^{n}$ is the state of the system, $u_{k} \in \mathbb{R}^{m}$ is the input, $y_{k} \in \mathbb{R}^{r}$ is the output, $\omega \in \mathbb{R}^{\Omega}$ is the constant input disturbance, and $y_{r e f} \in \mathbb{R}^{r}$ is a constant reference input, and assume that the system is open-loop asymptotically stable. Then at steady state, on denoting $\lim _{k \rightarrow \infty} x_{k}, \lim _{k \rightarrow \infty} y_{k}, \lim _{k \rightarrow \infty} u_{k}$ by $x_{\infty}, y_{\infty}, u_{\infty}$ respectively, the system equations become

$$
\begin{aligned}
x_{\infty} & =A x_{\infty}+B u_{\infty}+E \omega \\
\Rightarrow \quad x_{\infty} & =(I-A)^{-1} B u_{\infty}+(I-A)^{-1} E \omega \\
y_{\infty} & =C x_{\infty}+F \omega \\
& =\left(C(I-A)^{-1} B\right) u_{\infty}+\left(F+C(I-A)^{-1} E\right) \omega
\end{aligned}
$$


where $\Gamma=C(I-A)^{-1} B$ is the steady-state tracking gain matrix, and $\Lambda=(F+C(I-$ $\left.A)^{-1} E\right)$ is the steady-state disturbance gain matrix. $\Gamma$ can be experimentally found using the following procedure $[21,26]$ :

\section{Experimental Determination of $\Gamma$}

1. Assume the output $y_{k}$ can be measured, the inputs $u_{k}$ can be excited and no disturbances $\omega$ are acting on the plant.

2. For $i=1,2, \ldots, m$, apply a constant input vector $u_{k}=\left[\begin{array}{llllll}0 & \ldots & 0 & \alpha_{i} & 0 & \ldots\end{array}\right]^{\top}$ to the plant (2.1), with $\alpha_{i} \neq 0$, and measure the corresponding steady-state output to obtain the vector $y_{k}=\left[\begin{array}{llll}\beta_{i}^{1} & \beta_{i}^{2} \ldots \beta_{i}^{r}\end{array}\right]^{\top}$.

3. Solve the equation for $\Gamma$ :

$$
\Gamma\left[\begin{array}{cccc}
\alpha_{1} & 0 & \ldots & 0 \\
0 & \alpha_{2} & \ldots & 0 \\
& & \ddots & \\
0 & 0 & \ldots & \alpha_{m}
\end{array}\right]=\left[\begin{array}{cccc}
\beta_{1}^{1} & \beta_{2}^{1} & \ldots & \beta_{m}^{1} \\
\beta_{1}^{2} & \beta_{2}^{2} & \ldots & \beta_{m}^{2} \\
& & \ddots & \\
\beta_{1}^{r} & \beta_{2}^{r} & \ldots & \beta_{m}^{r}
\end{array}\right]
$$

\section{Experimental Determination of $\Lambda$}

Similarly, $\Lambda$ can be experimentally found using the following procedure:

1. Assume the output $y_{k}$ can be measured, the disturbances $\omega$ can be measured and be excited, and no inputs $u_{k}$ are applied to the plant.

2. For $i=1,2, \ldots, \Omega$, apply a constant disturbance vector $\omega=\left[\begin{array}{llllll}0 & \ldots & 0 & \gamma_{i} & 0 & \ldots\end{array}\right]^{\top}$ to the plant $(2.1)$, with $\gamma_{i} \neq 0$, and measure the corresponding steady-state output to obtain the vector $y=\left[\begin{array}{llll}\rho_{i}^{1} & \rho_{i}^{2} & \ldots & \rho_{i}^{r}\end{array}\right]^{\top}$.

3. Solve the equation for $\Lambda$ :

$$
\Lambda\left[\begin{array}{cccc}
\gamma_{1} & 0 & \ldots & 0 \\
0 & \gamma_{2} & \ldots & 0 \\
& & \ddots & \\
0 & 0 & \ldots & \gamma_{\Omega}
\end{array}\right]=\left[\begin{array}{cccc}
\rho_{1}^{1} & \rho_{2}^{1} & \ldots & \rho_{\Omega}^{1} \\
\rho_{1}^{2} & \rho_{2}^{2} & \ldots & \rho_{\Omega}^{2} \\
& & \ddots & \\
\rho_{1}^{r} & \rho_{2}^{r} & \ldots & \rho_{\Omega}^{r}
\end{array}\right]
$$




\subsection{Tuning Regulator}

If $K_{P}$ in (2.6) is set to 0 , the resulting controller is of the form of a tuning regulator [21]:

$$
\begin{gathered}
\eta_{k+1}=\eta_{k}+h e_{k} \\
u_{k}=-\epsilon S \eta_{k}
\end{gathered}
$$

where $h$ is the sampling period, $S=\Gamma^{-1}$ and $\epsilon>0$. The tuning regulator possesses three appealing properties:

Property 2.1. The tuning regulator (2.17) has the properties that

1. it solves the robust servomechanism problem.

2. it can be implemented without knowledge of the plant model.

3. There exists an $\epsilon^{*}>0$ such that, for all $\epsilon \in\left(0, \epsilon^{*}\right]$ and $t \geq 0,\|u(t)\| \leq u_{\text {Sat }}$, i.e., the control input actuators will not saturate at all times.

Properties 1 and 2 were already discussed in Sections 2.2.1 and 2.2.2, respectively. We will prove property 3 under the continuous time domain setup. If property 3 holds for the continuous controller

$$
\begin{aligned}
& \dot{\eta}=e \\
& u=-\epsilon S \eta,
\end{aligned}
$$

then by continuity, an implementation of the continuous controller using a "small enough sampling period" will yield the same results.

To prove the non-saturating property, we will consider an equivalent form of controller (2.18) in terms of the control input $u$ perspective, i.e.,

$$
\begin{aligned}
& \dot{\eta}=\epsilon e \\
& u=-S \eta
\end{aligned}
$$

as it is in a form that will allow us to formulate the problem within a singular perturbation framework. The following theorem presents the singular perturbation result of interest. The following definition will be used in Theorem 2.1.

Definition $2.2([27]) \cdot \delta_{1}(\epsilon)=O\left(\delta_{2}(\epsilon)\right)$ if there exist positive constants $k$ and $\epsilon^{*}$ such that, for all $\epsilon \in\left[0, \epsilon^{*}\right)$,

$$
\left|\delta_{1}(\epsilon)\right| \leq k\left|\delta_{2}(\epsilon)\right| .
$$


Theorem 2.1 ([27]). Consider the following system where $\epsilon>0$ is a scalar

$$
\left[\begin{array}{c}
\frac{d x(t)}{d t} \\
\epsilon \frac{d z(t)}{d t}
\end{array}\right]=\left[\begin{array}{ll}
A_{1} & A_{2} \\
A_{3} & A_{4}
\end{array}\right]\left[\begin{array}{l}
x(t) \\
z(t)
\end{array}\right], \quad\left[\begin{array}{l}
x\left(t_{0}\right) \\
z\left(t_{0}\right)
\end{array}\right]=\left[\begin{array}{l}
x_{0} \\
z_{0}
\end{array}\right]
$$

where $A_{4}$ is asymptotically stable, and assume $\epsilon \rightarrow 0$. Define the reduced system to be

$$
\frac{d \bar{x}(t)}{d t}=A_{1} \bar{x}(t)+A_{2} \bar{z}(t), \quad \bar{x}\left(t_{0}\right)=x_{0}
$$

where

$$
\bar{z}(t)=-A_{4}{ }^{-1} A_{3} \bar{x}(t)
$$

is the quasi-steady-state of z. Furthermore, define the boundary-layer system to be

$$
\frac{d p(\tau)}{d \tau}=A_{4} p(\tau), \quad p(0)=z_{0}-\bar{z}\left(t_{0}\right) .
$$

Assume that the following conditions are satisfied:

1. The reduced system (2.22) is exponentially stable.

2. The boundary-layer system (2.24) is exponentially stable.

Then there is a positive constant $\epsilon^{*}$ such that for all $t_{0} \geq 0, \bar{x}\left(t_{0}\right), p(0)$, and $0<\epsilon<\epsilon^{*}$, the singular perturbation problem of (2.21) has a unique solution $x(t, \epsilon), z(t, \epsilon)$ on $\left[t_{0}, \infty\right)$, and

$$
\begin{gathered}
x(t, \epsilon)-\bar{x}(t)=O(\epsilon) \\
z(t, \epsilon)-\bar{z}(t)-p(t / \epsilon)=O(\epsilon)
\end{gathered}
$$

hold uniformly for $t \in\left[t_{0}, \infty\right)$, where $\bar{x}(t)$ and $p(\tau)$ are the solutions of the reducd and boundary-layer problems (2.22) and (2.24).

Now we proceed to the proof of property 2.1, result 3. A similar proof can be found in [28] where the tuning regulator is applied to positive LTI systems.

Proof. Consider the continuous time LTI plant

$$
\begin{aligned}
\dot{x}(t) & =A x(t)+B u(t)+E \omega \\
y(t) & =C x(t)+F \omega \\
e(t) & =y(t)-y_{r e f}
\end{aligned}
$$


and the tuning regulator

$$
\begin{aligned}
& \dot{\eta}(t)=\epsilon e(t) \\
& u(t)=-K \eta(t)
\end{aligned}
$$

where $K=\left(-C A^{-1} B\right)^{-1}, \epsilon>0$, and $A$ is assumed to be asymptotically stable. Applying controller (2.27) to plant (2.26) gives rise to the following closed-loop augmented plant:

$$
\left[\begin{array}{l}
\dot{x}(t) \\
\dot{\eta}(t)
\end{array}\right]=\left[\begin{array}{cc}
A & -B K \\
\epsilon C & 0
\end{array}\right]\left[\begin{array}{l}
x(t) \\
\eta(t)
\end{array}\right]+\left[\begin{array}{cc}
E & 0 \\
\epsilon F & -\epsilon
\end{array}\right]\left[\begin{array}{c}
\omega \\
y_{r e f}
\end{array}\right], \quad\left[\begin{array}{l}
x(0) \\
\eta(0)
\end{array}\right] \triangleq\left[\begin{array}{l}
x_{0} \\
\eta_{0}
\end{array}\right] .
$$

Define

$$
\left[\begin{array}{l}
z(t) \\
q(t)
\end{array}\right]=\left[\begin{array}{l}
x(t) \\
\eta(t)
\end{array}\right]-\left[\begin{array}{l}
x(\infty) \\
\eta(\infty)
\end{array}\right]
$$

system (2.28) becomes

$$
\left[\begin{array}{c}
\dot{z}(t) \\
\dot{q}(t)
\end{array}\right]=\left[\begin{array}{cc}
A & -B K \\
\epsilon C & 0
\end{array}\right]\left[\begin{array}{l}
z(t) \\
q(t)
\end{array}\right], \quad\left[\begin{array}{l}
z(0) \\
q(0)
\end{array}\right]=\left[\begin{array}{l}
x_{0}-x(\infty) \\
\eta_{0}-\eta(\infty)
\end{array}\right] \triangleq\left[\begin{array}{l}
z_{0} \\
q_{0}
\end{array}\right] .
$$

(2.29) can be re-written as

$$
\begin{aligned}
{\left[\begin{array}{c}
\dot{q}(t) \\
\dot{z}(t)
\end{array}\right] } & =\left[\begin{array}{cc}
0 & \epsilon C \\
-B K & A
\end{array}\right]\left[\begin{array}{l}
q(t) \\
z(t)
\end{array}\right] \\
\Rightarrow \quad\left[\begin{array}{c}
\frac{1}{\epsilon} \dot{q}(t) \\
\dot{z}(t)
\end{array}\right] & =\left[\begin{array}{cc}
0 & C \\
-B K & A
\end{array}\right]\left[\begin{array}{l}
q(t) \\
z(t)
\end{array}\right] .
\end{aligned}
$$

Let $\frac{1}{\epsilon} \frac{d(\cdot)}{d t}=\frac{d(\cdot)}{d \tau}=\stackrel{\odot}{(\cdot)}$, so that the system is finally expressed as

$$
\left[\begin{array}{c}
\stackrel{\odot}{q}(t) \\
\dot{\odot}(t)
\end{array}\right]=\left[\begin{array}{cc}
0 & C \\
-B K & A
\end{array}\right]\left[\begin{array}{l}
q(t) \\
z(t)
\end{array}\right]
$$

Our model has now been transformed into that of the singular perturbation model. We need to show that the reduced and boundary-layer models are exponentially stable. By setting $\epsilon=0$ we obtain

$$
\begin{aligned}
0 & =-B K q(t)+A z(t) \\
\Rightarrow \quad z(t) & =h(t, q(t))=A^{-1} B K q(t) \\
\stackrel{\odot}{q}(t) & =C A^{-1} B K q(t) \\
& =\left(C A^{-1} B\right)\left(-C A^{-1} B\right)^{-1} q(t) \\
& =-q(t) .
\end{aligned}
$$


The expression for $q(t)$ can be obtained through integration:

$$
\begin{aligned}
\frac{d q(t)}{d \tau} & =-q(t) \\
\Rightarrow \quad \int_{q_{0}}^{q(t)} \frac{d q(t)}{q(t)} & =-\int_{0}^{\tau} d \tau \\
\Rightarrow \quad \ln q(t) & =-\tau+\ln q_{0},
\end{aligned}
$$

which implies that

$$
\begin{aligned}
& q(t)=e^{-\epsilon t} q_{0} \\
& z(t)=A^{-1} B K e^{-\epsilon t} q_{0} .
\end{aligned}
$$

Let the solutions of $q(t)$ and $z(t)$ obtained above in (2.34) be denoted as $\bar{q}(t)$ and $\bar{z}(t)$, respectively. These are the solutions to the reduced model, which is obtained on setting $\epsilon$ to 0 . This model is exponentially stable. To obtain the boundary-layer model, we perform the change of variables $p(t)=z(t)-h(t, q(t))$ that shifts the quasi-steady-state of $z$ to the origin. In the new variables $(q(t), p(t))$, the full problem is

$$
\begin{aligned}
\stackrel{\odot}{q}(t) & =-D K q(t)+C z(t) \\
& =-D K q(t)+C p(t)+C h(t, q(t)) \\
\epsilon \dot{p}(t) & =\epsilon \stackrel{\odot}{z}(t)-\epsilon \stackrel{\odot}{h}(t, q(t)) .
\end{aligned}
$$

Recall

$$
\epsilon \frac{d(\cdot)}{d \tau}=\frac{d(\cdot)}{d t}=(\cdot)
$$

then

$$
\begin{aligned}
\frac{d p(t)}{d t} & =\frac{d z(t)}{d t}-\frac{d h(t, q(t))}{d t} \\
& =\dot{z}(t)-A^{-1} B K \dot{q}(t) \\
& =-B K q(t)+A z(t)-A^{-1} B K \dot{q}(t) \\
& =-B K q(t)+A z(t)-A^{-1} B K(-\epsilon D K q(t)+\epsilon C z(t)) \\
\left.\dot{p}(t)\right|_{\epsilon=0} & =-B K q(t)+A p(t)+B K q(t)+\left.A^{-1} B K(\epsilon D K q(t)-\epsilon C z(t))\right|_{\epsilon=0} \\
& =A p(t) .
\end{aligned}
$$

Since $A$ is assumed to be asymptotically stable, the origin $p(t)=0$ of the boundary-layer model is exponentially stable. The solution of (2.37) is

$$
\hat{p}(t)=e^{A t} p(0)=e^{A t}\left(z_{0}-A^{-1} B K q_{0}\right) .
$$


According to Theorem 2.1 we can conclude that there exists a time $t_{O(\epsilon)}$ such that, for all $t \geq t_{O(\epsilon)}$,

$$
\begin{aligned}
& q(t)-\bar{q}(t)=O(\epsilon) \\
\Rightarrow \quad & q(t)=e^{-\epsilon t} q_{0}+O(\epsilon) .
\end{aligned}
$$

Substituting back $q(t)=\eta(t)-\eta(\infty)$, we obtain

$$
\eta(t)=\eta(\infty)+e^{-\epsilon t}\left(\eta_{0}-\eta(\infty)\right)+O(\epsilon)
$$

Similarly, for all $t \geq t_{O(\epsilon)}$

$$
\begin{aligned}
z(t) & -\bar{z}(t)-\hat{p}(t)=O(\epsilon) \\
\Rightarrow \quad z(t) & =\bar{z}(t)+\hat{p}(t)+O(\epsilon) \\
& =A^{-1} B K e^{-\epsilon t} q_{0}+e^{A t}\left(z_{0}-A^{-1} B K q_{0}\right)+O(\epsilon) .
\end{aligned}
$$

Substituting back $z(t)=x(t)-x(\infty)$, we obtain

$$
\begin{aligned}
x(t)= & x(\infty)+A^{-1} B K e^{-\epsilon t}\left(\eta_{0}-\eta(\infty)\right) \\
& +e^{A t}\left(\left(x_{0}-x(\infty)\right)-A^{-1} B K\left(\eta_{0}-\eta(\infty)\right)\right)+O(\epsilon) .
\end{aligned}
$$

The $O(\epsilon)$ terms in equations (2.40) and (2.42) capture the transient peaking and/or overshoot portions of the $\eta(t)$ and $x(t)$ signals. This means that the amount of peaking or overshoot diminishes as we make $\epsilon$ smaller and smaller. In other words, $[x(t) \eta(t)]^{\top}$ can be made to approach $[x(\infty) \quad \eta(\infty)]^{\top}$ monotonically, with no overshoot, but at the cost of a slower system response. It should be noted that the dependency on $x_{0}$ for $\eta(t)$ is encapsulated in the $O(\epsilon)$ term in (2.40). Since $u(t)=K \eta(t)$, if $\left[\begin{array}{llll}x_{0} & \eta_{0} & \omega & y_{\text {ref }}\end{array}\right]^{\top}$ is chosen such that $\|u(\infty)\| \leq u_{\text {Sat }}$ and a sufficiently small $\epsilon$ is used, saturation will not occur for all $t \geq 0$.

\subsection{Controller Parameter Selection}

Even though small values of $K_{P}$ and $K_{I}$ guarantee closed-loop stability, the system response can be excessively slow. Motivated by the optimal transient response shaping results in $[29,30]$, we propose that $K_{P}$ and $K_{I}$ are to be chosen by solving one of the two parameter optimization problems presented below, which will be referred to as optimization problem $A$ and optimization problem $B$. 


\subsubsection{Optimization Problem A}

Consider cascading the servocompensator (2.4) with the plant (2.1) to form an augmented plant given by

$$
\begin{aligned}
{\left[\begin{array}{l}
x_{k+1} \\
\eta_{k+1}
\end{array}\right] } & =\left[\begin{array}{cc}
A & 0 \\
h C & I
\end{array}\right]\left[\begin{array}{l}
x_{k} \\
\eta_{k}
\end{array}\right]+\left[\begin{array}{c}
B \\
0
\end{array}\right] u_{k}+\left[\begin{array}{cc}
E & 0 \\
h F & -h
\end{array}\right]\left[\begin{array}{c}
\omega \\
y_{r e f}
\end{array}\right] \\
z_{k} & =\left[\begin{array}{ll}
\theta C & I
\end{array}\right]\left[\begin{array}{l}
x_{k} \\
\eta_{k}
\end{array}\right]
\end{aligned}
$$

where $\theta$ is a non-negative scalar value. Now, let

$$
\Delta x_{k} \triangleq x_{k}-x_{k-1}, \quad \Delta \eta_{k} \triangleq \eta_{k}-\eta_{k-1}, \quad \Delta u_{k} \triangleq u_{k}-u_{k-1}, \quad \Delta z_{k} \triangleq z_{k}-z_{k-1} .
$$

Then, on substituting (2.44) into (2.43), we obtain the following equivalent representation of $(2.43)$ :

$$
\begin{aligned}
{\left[\begin{array}{c}
\Delta x_{k+1} \\
\Delta \eta_{k+1}
\end{array}\right] } & =\left[\begin{array}{cc}
A & 0 \\
h C & I
\end{array}\right]\left[\begin{array}{c}
\Delta x_{k} \\
\Delta \eta_{k}
\end{array}\right]+\left[\begin{array}{c}
B \\
0
\end{array}\right] \Delta u_{k} \\
\Delta z_{k} & =\left[\begin{array}{ll}
\theta C & I
\end{array}\right]\left[\begin{array}{l}
\Delta x_{k} \\
\Delta \eta_{k}
\end{array}\right] .
\end{aligned}
$$

The $K_{P}$ and $K_{I}$ parameters will be chosen so as to minimize the performance index

$$
J_{A} \triangleq \frac{1}{h} \sum_{k=1}^{\infty}\left(\Delta z_{k}\right)^{\top}\left(\Delta z_{k}\right)+\mu\left(\Delta u_{k}\right)^{\top}\left(\Delta u_{k}\right), \quad \mu>0
$$

subject to (2.45) and (2.46). Substituting the controller (2.6) into (2.45) yields the closed-loop equations

$$
\begin{aligned}
\tilde{x}_{k+1} & =\tilde{\mathcal{A}} \tilde{x}_{k} \\
\Delta z_{k} & =\tilde{\mathcal{C}}_{z} \tilde{x}_{k} \\
\Delta u_{k} & =\tilde{\mathcal{C}}_{u} \tilde{x}_{k}
\end{aligned}
$$

where

$$
\begin{aligned}
\tilde{\mathcal{A}} & =\left[\begin{array}{cc}
A-B S K_{P} C & -B S K_{I} \\
h C & I
\end{array}\right] \\
\tilde{\mathcal{C}}_{z} & =\left[\begin{array}{ll}
\theta C & I
\end{array}\right] \\
\tilde{\mathcal{C}}_{u} & =\left[\begin{array}{ll}
-S K_{P} C & -S K_{I}
\end{array}\right] \\
\tilde{x}_{k} & :=\left[\begin{array}{l}
\Delta x_{k} \\
\Delta \eta_{k}
\end{array}\right]=\left[\begin{array}{l}
x_{k}-x_{k-1} \\
\eta_{k}-\eta_{k-1}
\end{array}\right] .
\end{aligned}
$$


With the sampling index $k$ starting from $1, \Delta z_{k}$ and $\Delta u_{k}$ in (2.48) can be expressed as

$$
\begin{aligned}
\Delta z_{k} & =\tilde{\mathcal{C}}_{z} \tilde{\mathcal{A}}^{k-1} \tilde{x}_{1} \\
\Delta u_{k} & =\tilde{\mathcal{C}}_{u} \tilde{\mathcal{A}}^{k-1} \tilde{x}_{1} .
\end{aligned}
$$

Next, on substituting the $\Delta z_{k}$ and $\Delta u_{k}$ from (2.50) into performance index (2.47), we obtain

$$
J_{A}=\frac{1}{h} \tilde{x}_{1}^{\top}\left[\sum_{k=1}^{\infty}\left(\tilde{\mathcal{A}}^{\top}\right)^{k-1}\left(\tilde{\mathcal{C}}_{z}^{\top} \tilde{\mathcal{C}}_{z}+\mu \tilde{\mathcal{C}}_{u}^{\top} \tilde{\mathcal{C}}_{u}\right)(\tilde{\mathcal{A}})^{k-1}\right] \tilde{x}_{1} .
$$

If we apply the change of variables $i=k-1,(2.51)$ becomes

$$
J_{A}=\frac{1}{h} \tilde{x}_{0}^{\top}\left[\sum_{i=0}^{\infty}\left(\tilde{\mathcal{A}}^{\top}\right)^{i}\left(\tilde{\mathcal{C}}_{z}^{\top} \tilde{\mathcal{C}}_{z}+\mu \tilde{\mathcal{C}}_{u}^{\top} \tilde{\mathcal{C}}_{u}\right)(\tilde{\mathcal{A}})^{i}\right] \tilde{x}_{0}
$$

or

$$
J_{A}=\frac{1}{h} \tilde{x}_{0}^{\top} \tilde{\mathcal{P}} \tilde{x}_{0}
$$

where $\tilde{\mathcal{P}}>0$ is the solution of the discrete Lyapunov equation

$$
\tilde{\mathcal{A}}^{\top} \tilde{\mathcal{P}} \tilde{\mathcal{A}}-\tilde{\mathcal{P}}+\left(\tilde{\mathcal{C}}_{z}^{\top} \tilde{\mathcal{C}}_{z}+\mu \tilde{\mathcal{C}}_{u}^{\top} \tilde{\mathcal{C}}_{u}\right)=0 .
$$

The initial condition in (2.53) can be expressed as

$$
\tilde{x}_{0}=\left[\begin{array}{cc}
\left(A-B S K_{P} C\right)-I & -B S K_{I} \\
h C & 0
\end{array}\right]\left[\begin{array}{l}
x_{0} \\
\eta_{0}
\end{array}\right]+\left[\begin{array}{cc}
E-B S K_{P} F & 0 \\
h F & -h
\end{array}\right]\left[\begin{array}{c}
\omega \\
y_{r e f}
\end{array}\right] .
$$

From (2.53), we can define the following performance indices:

- Assume $\left(y_{0} \neq 0, \eta_{0}=0, \omega=0, y_{r e f}=0\right)$ :

$$
J_{A y_{0}}=\frac{1}{h} \times \operatorname{trace}\left(\left[\left(\left(A-B S K_{P} C\right)-I\right)^{\top} h C^{\top}\right] \tilde{\mathcal{P}}\left[\begin{array}{c}
\left(A-B S K_{P} C\right)-I \\
h C
\end{array}\right]\right) .
$$

This performance index measures the "average cost" of (2.47) over all initial conditions $y_{0}$ uniformly distributed on a unit disk ball [31]. Likewise, we can define the following performance indices:

- Assume $\left(x_{0}=0, \eta_{0} \neq 0, \omega=0, y_{\text {ref }}=0\right)$ :

$$
J_{A \eta_{0}}=\frac{1}{h} \times \operatorname{trace}\left(\left[\left(-B S K_{I}\right)^{\top} 0\right] \tilde{\mathcal{P}}\left[\begin{array}{c}
-B S K_{I} \\
0
\end{array}\right]\right) .
$$


- Assume $\left(x_{0}=0, \eta_{0}=0, \omega \neq 0, y_{\text {ref }}=0\right)$ :

$$
J_{A \omega}=\frac{1}{h} \times \operatorname{trace}\left(\left[\begin{array}{ll}
\left(E-B S K_{P} F\right)^{\top} & h F^{\top}
\end{array}\right] \tilde{\mathcal{P}}\left[\begin{array}{c}
\left(E-B S K_{P} F\right) \\
h F
\end{array}\right]\right) .
$$

- Assume $\left(x_{0}=0, \eta_{0}=0, \omega=0, y_{r e f} \neq 0\right)$ :

$$
\begin{aligned}
J_{A y_{\text {ref }}} & =\frac{1}{h} \times \operatorname{trace}\left(\left[\begin{array}{ll}
0 & -h
\end{array}\right] \tilde{\mathcal{P}}\left[\begin{array}{c}
0 \\
-h
\end{array}\right]\right) \\
& =h \times \operatorname{trace}\left(\left[\begin{array}{ll}
0 & I
\end{array}\right] \tilde{\mathcal{P}}\left[\begin{array}{l}
0 \\
I
\end{array}\right]\right) .
\end{aligned}
$$

The motivation for choosing such performance indices can be understood by first substituting the $\Delta \eta_{k+1}=h C \Delta x_{k}+\Delta \eta_{k}$ equation from (2.45) into (2.46) to give

$$
\Delta z_{k}=\frac{\theta}{h}\left(\Delta \eta_{k+1}-\Delta \eta_{k}\right)+\Delta \eta_{k}
$$

On noting that $\Delta \eta_{k}=h e_{k-1},(2.60)$ can then be re-written as

$$
\begin{aligned}
\Delta z_{k} & =\theta\left(e_{k}-e_{k-1}\right)+h e_{k-1} \\
& =\theta \Delta e_{k}+h e_{k-1} .
\end{aligned}
$$

If $\Delta z_{k}=0$, this implies

$$
\begin{aligned}
e_{k} & =\left(1-\frac{h}{\theta}\right) e_{k-1} \\
\Rightarrow \quad e_{k} & =\left(1-\frac{h}{\theta}\right)^{k} e_{0},
\end{aligned}
$$

i.e., the error response has a smooth, noninteracting decaying exponential type of behavior whose time constant is set by the $\theta$ value. Note that (2.62) can also be obtained by sampling $e(t)=\exp \left(-\theta^{-1} t\right) e(0)$ at $t=k h$ and using the exponential series approximation of $\exp \left(-\theta^{-1} t\right) \approx I-h \theta^{-1}[29]$.

In addition to having the desired transient response characteristics embedded within it, the performance index (2.47) has the property that if a stabilizing compensator is applied to a minimum phase plant as to minimize (2.47), the eigenvalues of the controlled system will either approach the origin or the transmission zeros of the plant as $\mu \rightarrow 0$. This may not be true for other performance indices such as

$$
J=\sum_{k=1}^{\infty}\left(\Delta e_{k}\right)^{\top}\left(\Delta e_{k}\right)+\mu\left(\Delta u_{k}\right)^{\top}\left(\Delta u_{k}\right)
$$


since the system

$$
\left\{\left[\begin{array}{cc}
C & 0
\end{array}\right],\left[\begin{array}{cc}
A & 0 \\
h C & I
\end{array}\right],\left[\begin{array}{l}
B \\
0
\end{array}\right], 0\right\}
$$

is nonminum phase with transmission zeros at $z=1$. As $\mu \rightarrow 0$, some closed-loop eigenvalues will approach $z=1$, which means the system becomes closer to becoming a marginally stable system [25].

The reason for having a $1 / h$ factor in the performance index $(2.47)$ can be understood by substituting the output expression $\Delta z_{k}$ from (2.61) into (2.47). If we assume $\theta$ and $\mu$ to be 0 , the performance index simplifies to

$$
\begin{aligned}
J_{A} & =\frac{1}{h} \sum_{k=1}^{\infty}\left(h e_{k-1}\right)^{\top}\left(h e_{k-1}\right) \\
& =h \sum_{k=1}^{\infty}\left(e_{k-1}\right)^{\top}\left(e_{k-1}\right) .
\end{aligned}
$$

Hence, we obtain the Riemann sum approximation of

$$
J=\int_{0}^{\infty} e^{\top} e d \tau .
$$

\subsubsection{Optimization Problem B}

Reconsider cascading now the servocompensator (2.4) with the plant (2.1) to form an augmented plant given by

$$
\begin{aligned}
{\left[\begin{array}{l}
x_{k+1} \\
\eta_{k+1}
\end{array}\right] } & =\left[\begin{array}{cc}
A & 0 \\
h C & I
\end{array}\right]\left[\begin{array}{l}
x_{k} \\
\eta_{k}
\end{array}\right]+\left[\begin{array}{l}
B \\
0
\end{array}\right] u_{k}+\left[\begin{array}{cc}
E & 0 \\
h F & -h
\end{array}\right]\left[\begin{array}{c}
\omega \\
y_{\text {ref }}
\end{array}\right] \\
z_{k} & =\left[\begin{array}{ll}
\theta C & I
\end{array}\right]\left[\begin{array}{l}
x_{k} \\
\eta_{k}
\end{array}\right]
\end{aligned}
$$

where $\theta$ is a non-negative scalar value. Now, let

$$
\Delta x_{k} \triangleq x_{k}-x_{\infty}, \quad \Delta \eta_{k} \triangleq \eta_{k}-\eta_{\infty}, \quad \Delta u_{k} \triangleq u_{k}-u_{\infty}, \quad \Delta z_{k} \triangleq z_{k}-z_{\infty}
$$

Then, on substituting (2.67) into (2.66), we obtain the following equivalent representation of (2.66):

$$
\begin{aligned}
{\left[\begin{array}{c}
\Delta x_{k+1} \\
\Delta \eta_{k+1}
\end{array}\right] } & =\left[\begin{array}{cc}
A & 0 \\
h C & I
\end{array}\right]\left[\begin{array}{c}
\Delta x_{k} \\
\Delta \eta_{k}
\end{array}\right]+\left[\begin{array}{c}
B \\
0
\end{array}\right] \Delta u_{k} \\
\Delta z_{k} & =\left[\begin{array}{ll}
\theta C & I
\end{array}\right]\left[\begin{array}{l}
\Delta x_{k} \\
\Delta \eta_{k}
\end{array}\right] .
\end{aligned}
$$


The $K_{P}$ and $K_{I}$ parameters will be chosen so as to minimize the performance index

$$
J_{B} \triangleq h \sum_{k=0}^{\infty}\left(\Delta z_{k}\right)^{\top}\left(\Delta z_{k}\right)+\mu\left(\Delta u_{k}\right)^{\top}\left(\Delta u_{k}\right), \quad \mu>0
$$

subject to (2.68) and (2.69). On substituting the controller (2.6) into (2.68) we obtain the closed-loop equations

$$
\begin{aligned}
\bar{x}_{k+1} & =\overline{\mathcal{A}} \bar{x}_{k} \\
\Delta z_{k} & =\overline{\mathcal{C}}_{z} \bar{x}_{k} \\
\Delta u_{k} & =\overline{\mathcal{C}}_{u} \bar{x}_{k}
\end{aligned}
$$

where

$$
\begin{aligned}
\overline{\mathcal{A}} & =\left[\begin{array}{cc}
A-B S K_{P} C & -B S K_{I} \\
h C & I
\end{array}\right] \\
\overline{\mathcal{C}}_{z} & =\left[\begin{array}{ll}
\theta C & I
\end{array}\right] \\
\overline{\mathcal{C}}_{u} & =\left[\begin{array}{ll}
-S K_{P} C & -S K_{I}
\end{array}\right] \\
\bar{x}_{k} & :=\left[\begin{array}{l}
\Delta x_{k} \\
\Delta \eta_{k}
\end{array}\right]=\left[\begin{array}{l}
x_{0}-x_{\infty} \\
\eta_{0}-\eta_{\infty}
\end{array}\right] .
\end{aligned}
$$

With the sampling index $k$ starting from $0, \Delta z_{k}$ and $\Delta u_{k}$ in (2.71) can be expressed as

$$
\begin{aligned}
\Delta z_{k} & =\overline{\mathcal{C}}_{z} \overline{\mathcal{A}}^{k} \bar{x}_{0} \\
\Delta u_{k} & =\overline{\mathcal{C}}_{u} \overline{\mathcal{A}}^{k} \bar{x}_{0}
\end{aligned}
$$

and on substituting $\Delta z_{k}$ and $\Delta u_{k}$ from (2.73) into performance index (2.70), yields

$$
J_{B}=h \bar{x}_{0}^{\top}\left[\sum_{k=0}^{\infty}\left(\overline{\mathcal{A}}^{\top}\right)^{k}\left(\overline{\mathcal{C}}_{z}^{\top} \overline{\mathcal{C}}_{z}+\mu \overline{\mathcal{C}}_{u}^{\top} \overline{\mathcal{C}}_{u}\right)(\overline{\mathcal{A}})^{k}\right] \bar{x}_{0}
$$

or

$$
J_{B}=h \bar{x}_{0}^{\top} \overline{\mathcal{P}} \bar{x}_{0}
$$

where $\overline{\mathcal{P}}>0$ is the solution of the discrete Lyapunov equation

$$
\overline{\mathcal{A}}^{\top} \overline{\mathcal{P}} \overline{\mathcal{A}}-\overline{\mathcal{P}}+\left(\overline{\mathcal{C}}_{z}^{\top} \overline{\mathcal{C}}_{z}+\mu \overline{\mathcal{C}}_{u}^{\top} \overline{\mathcal{C}}_{u}\right)=0
$$

The initial condition in (2.75) can be expressed as (see Appendix A.2 for derivation)

$$
\bar{x}_{0}=\left[\begin{array}{l}
x_{0} \\
\eta_{0}
\end{array}\right]-\left[\begin{array}{cc}
I-\left(A-B S K_{P} C\right) & B S K_{I} \\
-h C & 0
\end{array}\right]^{-1}\left[\begin{array}{cc}
E-B S K_{P} F & 0 \\
h F & -h
\end{array}\right]\left[\begin{array}{c}
\omega \\
y_{r e f}
\end{array}\right] .
$$

From (2.75), we can now define the following performance indices: 
- Assume $\left(y_{0} \neq 0, \eta_{0}=0, \omega=0, y_{\text {ref }}=0\right)$ :

$$
J_{B y_{0}}=h \times \operatorname{trace}\left(\left[\begin{array}{ll}
I & 0
\end{array}\right] \overline{\mathcal{P}}\left[\begin{array}{l}
I \\
0
\end{array}\right]\right) \text {. }
$$

- Assume $\left(x_{0}=0, \eta_{0} \neq 0, \omega=0, y_{\text {ref }}=0\right)$ :

$$
J_{B \eta_{0}}=h \times \operatorname{trace}\left(\left[\begin{array}{ll}
0 & I
\end{array}\right] \overline{\mathcal{P}}\left[\begin{array}{l}
0 \\
I
\end{array}\right]\right) .
$$

- Assume $\left(x_{0}=0, \eta_{0}=0, \omega \neq 0, y_{\text {ref }}=0\right)$ :

$$
\begin{aligned}
J_{B \omega}= & h \times \operatorname{trace}\left(\left(\left[\begin{array}{rr}
I-\left(A-B S K_{P} C\right) & B S K_{I} \\
-h C & 0
\end{array}\right]^{-1}\left[\begin{array}{c}
E-B S K_{P} F \\
h F
\end{array}\right]\right)\right)^{\top} \times \\
& \left.\overline{\mathcal{P}} \times\left(\left[\begin{array}{cc}
I-\left(A-B S K_{P} C\right) & B S K_{I} \\
-h C & 0
\end{array}\right]^{-1}\left[\begin{array}{c}
E-B S K_{P} F \\
h F
\end{array}\right]\right)\right) .
\end{aligned}
$$

- Assume $\left(x_{0}=0, \eta_{0}=0, \omega=0, y_{\text {ref }} \neq 0\right)$ :

$$
\begin{aligned}
J_{B y_{r e f}=}= & h \operatorname{trace}\left(\left(\left[\begin{array}{cc}
I-\left(A-B S K_{P} C\right) & B S K_{I} \\
-h C & 0
\end{array}\right]^{-1}\left[\begin{array}{c}
0 \\
-h
\end{array}\right]\right){ }^{\top} \times\right. \\
& \left.\overline{\mathcal{P}} \times\left(\left[\begin{array}{cc}
I-\left(A-B S K_{P} C\right) & B S K_{I} \\
-h C & 0
\end{array}\right]^{-1}\left[\begin{array}{c}
0 \\
-h
\end{array}\right]\right)\right) .
\end{aligned}
$$

The motivation for choosing such a performance index and output $\Delta z_{k}$ can be understood by first substituting the $\Delta \eta_{k+1}=h C \Delta x_{k}+\Delta \eta_{k}$ equation from (2.68) into (2.69) to give

$$
\Delta z_{k}=\frac{\theta}{h}\left(\Delta \eta_{k+1}-\Delta \eta_{k}\right)+\Delta \eta_{k}
$$

Notice that

$$
\begin{aligned}
& \eta_{k+1}-\eta_{k}=h e_{k} \\
\Rightarrow \quad & \left(\eta_{k+1}-\eta_{\infty}\right)-\left(\eta_{k}-\eta_{\infty}\right)=h e_{k} \\
\Rightarrow & \Delta \eta_{k+1}-\Delta \eta_{k}=h e_{k} .
\end{aligned}
$$

Thus, output (2.82) can also be expressed as

$$
\Delta z_{k}=\theta e_{k}+\Delta \eta_{k}
$$


If $\Delta z_{k}=0$, this means that (2.82) becomes

$$
\begin{aligned}
& \Delta \eta_{k+1}=\left(1-\frac{h}{\theta}\right) \Delta \eta_{k} \\
\Rightarrow \quad & \Delta \eta_{k}=\left(1-\frac{h}{\theta}\right)^{k} \Delta \eta_{0} .
\end{aligned}
$$

Substituting (2.86) into (2.84) yields

$$
e_{k}=-\frac{1}{\theta}\left(1-\frac{h}{\theta}\right)^{k} \Delta \eta_{0}
$$

This means that the ideal error response has a smooth, noninteracting decaying exponential type of behavior whose time constant is set by the $\theta$ value. Note that (2.87) can be obtained by sampling $e(t)=-\theta^{-1} \exp \left(-\theta^{-1} t\right) \Delta \eta(0)$ at $t=k h$ and using the approximation of $\exp \left(-\theta^{-1} t\right) \approx I-h \theta^{-1}$. The desirable properties of performance index (2.47) discussed in Section 2.4.1 hold for (2.70) as well.

\subsection{Proposed Controller Tuning Approach}

\subsubsection{Tuning for Tracking Performance}

Since the closed-loop matrices $\left(\tilde{\mathcal{A}}, \tilde{\mathcal{C}}_{z}, \tilde{\mathcal{C}}_{z}\right)$ in $(2.49)$ are identical to the closed-loop matrices $\left(\overline{\mathcal{A}}, \overline{\mathcal{C}}_{z}, \overline{\mathcal{C}}_{z}\right)$ in $(2.72)$ for given fixed values of $K_{P}, K_{I}, \theta$ and $\mu$, solving the Lyapunov equation under either optimization problem $A(2.54)$ or $B(2.76)$ yields the same solution. This implies that minimizing the performance index $J_{A y_{r e f}}(2.59)$ will yield the same optimal controller as minimizing $J_{B \eta_{0}}(2.79)$. In other words, we can indirectly tune the controller for optimal tracking performance by perturbing only the states of the servocompensator $\eta_{0}$ while minimizing performance index $J_{B}(2.70)$. This approach is advantageous as the tuning process can then be carried out without perturbing the plant's initial states or changing the plant's tracking set-points.

\subsubsection{Tuning for Disturbance Rejection Performance}

If the disturbance signal $\omega$ can be excited, one can tune the controller for optimal disturbance rejection performance by minimizing the performance index $J_{A \omega}(2.58)$ or $J_{B \omega}$ (2.80). However, for many systems or processes $\omega$ can not be measured nor excited. For these systems, we propose to first tune the controller for tracking performance and have no disturbances acting upon the system during the optimization process, then apply this 
same controller for disturbance rejection. It can be shown that a controller which is tuned for tracking will also perform well for disturbance rejection. For example, consider the following scalar 2 input and 2 output system:

$$
\begin{aligned}
x_{k+1} & =A x_{k}+B u_{k} \\
y_{k} & =C x_{k} \\
e_{k} & =C x_{k}-y_{r e f}
\end{aligned}
$$

where disturbance $\omega=0$ and $y_{\text {ref }} \neq 0$. As previously mentioned, the parameters of the two-term controller (2.6) for this system can be tuned by minimizing the performance index

$$
J_{A}=\frac{1}{h} \sum_{k=1}^{\infty}\left(h e_{k-1}\right)^{\top}\left(h e_{k-1}\right)+\mu\left(u_{k}-u_{k-1}\right)^{\top}\left(u_{k}-u_{k-1}\right)
$$

over a representative class of $y_{\text {ref }}$ signals distributed on the unit disk, e.g., $y_{\text {ref }}=\left[\begin{array}{ll}1 & 0\end{array}\right]^{\top}$, $y_{\text {ref }}=\left[\begin{array}{ll}0 & 1\end{array}\right]^{\top}$. The designed controller in this case

$$
u_{k}=-S\left(K_{\text {Popt }} y_{k}+K_{\text {Iopt }} \eta_{k}\right)
$$

will perform well for the tracking signals $y_{\text {ref }}=\left[\begin{array}{ll}1 & 0\end{array}\right]^{\top}, y_{\text {ref }}=\left[\begin{array}{ll}0 & 1\end{array}\right]^{\top}$, and the linear combination $y_{r e f}=a\left[\begin{array}{ll}1 & 0\end{array}\right]^{\top}+b\left[\begin{array}{ll}0 & 1\end{array}\right]^{\top}$ for all $a, b \in \mathbb{R}$. Now, consider the system

$$
\begin{aligned}
x_{k+1} & =A x_{k}+B u_{k} \\
y_{k} & =C x_{k}+F \omega \\
e_{k} & =C x_{k}+F \omega
\end{aligned}
$$

where $\omega \neq 0, y_{\text {ref }}=0$, and assume the disturbance term $F \omega \neq 0$. In this case, $F \omega$ is a $2 \times 1$ column vector that can be expressed as a linear combination of the vectors $\left[\begin{array}{ll}1 & 0\end{array}\right]^{\top}$ and $\left[\begin{array}{ll}0 & 1\end{array}\right]^{\top}$, i.e., the error $e_{k}$ in (2.88) and (2.91) have the same form; thus, the controller (2.90) obtained from minimizing the error signal will also perform well for disturbance rejection. If the system has a state disturbance term, i.e., the system is described by

$$
\begin{aligned}
x_{k+1} & =A x_{k}+B u_{k}+E \omega \\
y_{k} & =C x_{k} \\
e_{k} & =C x_{k}
\end{aligned}
$$


where $\omega \neq 0$ and $y_{\text {ref }}=0$, we can apply the following transformations to recast (2.92) into the form of (2.91): Let $x_{k}=\bar{x}+\Delta x_{k}$ and substitute it into (2.92) to obtain

$$
\begin{aligned}
\Delta x_{k+1} & =A \Delta x_{k}+B u_{k}+E \omega+(A-I) \bar{x} \\
y_{k} & =C \Delta x_{k}+C \bar{x} \\
e_{k} & =C \Delta x_{k}+C \bar{x} .
\end{aligned}
$$

Furthermore, let $\bar{x}=(I-A)^{-1} E \omega$ and we obtain

$$
\begin{aligned}
\Delta x_{k+1} & =A \Delta x_{k}+B u_{k} \\
y_{k} & =C \Delta x_{k}+C(I-A)^{-1} E \omega \\
e_{k} & =C \Delta x_{k}+C(I-A)^{-1} E \omega
\end{aligned}
$$

where $C(I-A)^{-1} E$ is the new $F$ matrix. Systems (2.91) and (2.94) are now in the same form; thus, a controller which is tuned for tracking will also have good disturbance rejection performance for these classes of systems.

\subsubsection{Using a Non-Noise Sensitive Performance Index}

Choosing the optimization problem $B$ method over $A$ has the additional advantage that performance index $J_{B}(2.70)$ is less sensitive to random error (i.e., noise) that can arise as a result of quantization effects, or unpredictable and unrepeatable fluctuations from experiments. This can be illustrated by the following analysis on the structure of performance index $J_{A}(2.47)$ where we reconsider the performance index of optimization problem A given by

$$
J_{A}=\frac{1}{h} \sum_{k=1}^{\infty}\left(\Delta z_{k}\right)^{\top}\left(\Delta z_{k}\right)+\mu\left(\Delta u_{k}\right)^{\top}\left(\Delta u_{k}\right)
$$

where $\Delta z_{k}=\theta\left(e_{k}-e_{k-1}\right)+h e_{k-1}$ and $\Delta u_{k}=u_{k}-u_{k-1}$. When noise is present, large values for the disturbance signal $\omega$ and tracking reference signal $y_{\text {ref }}$ may be required to obtain good signal-to-noise ratio of the measurable signals for computing $J_{A}$ due to the structure of $\Delta u_{k}$ and $\Delta z_{k}$. This can be illustrated by first assuming a simple controller of the form is used:

$$
u_{k}=K\left(y_{k}+W_{k}\right)
$$

where $W_{k}$ is a white Gaussian noise with zero mean and finite variance. Then

$$
\Delta u_{k}=u_{k}-u_{k-1}=K\left(y_{k}-y_{k-1}\right)+K\left(W_{k}-W_{k-1}\right)
$$


where $W_{k}$ and $W_{k-1}$ are independent and identical distributed random variables. According to the Bienaymé equality [32], which states that the variance of a sum of independent random variables is equal to the sum of their variances, this implies that $W_{k}-W_{k-1}$ is a white Gaussian noise with twice the variance of $W_{k}$ and the noise is further amplified by a factor of $K$. A similar analysis can be done to show that the $W_{k}-W_{k-1}$ term is also present in the expression for $\Delta z_{k}$ and plays a role if $\theta \neq 0$. This implies that it becomes more difficult to obtain reliable cost values as the controller gains increase when solving optimization problem A.

\subsection{Controller Comparison}

If we choose our servomechanism controller to be

$$
u_{k}=-S\left(K_{P} e_{k}+K_{I} \eta_{k}\right)
$$

instead of the controller (2.6)

$$
u_{k}=-S\left(K_{P} y_{k}+K_{I} \eta_{k}\right)
$$

the performance index $J_{A y_{r e f}}(2.59)$ can be reexpressed as

$$
\overline{J_{A y_{r e f}}}:=J_{A}=\frac{1}{h} \times \operatorname{trace}\left(\left[\left(B S K_{P}\right)^{\top}-h\right] \tilde{\mathcal{P}}\left[\begin{array}{c}
B S K_{P} \\
-h
\end{array}\right]\right)
$$

while the performance index $J_{B \eta_{0}}(2.79)$ remains to be the same

$$
\overline{J_{B \eta_{0}}}=J_{B \eta_{0}}:=J_{B}=h \times \operatorname{trace}\left(\left[\begin{array}{ll}
0 & I
\end{array}\right] \overline{\mathcal{P}}\left[\begin{array}{l}
0 \\
I
\end{array}\right]\right) .
$$

Minimizing $\overline{J_{A y_{r e f}}}(2.99)$ and $\overline{J_{B \eta_{0}}}(2.100)$ gives rise to different optimal controllers even if the $\tilde{\mathcal{P}}$ and $\overline{\mathcal{P}}$ matrices are the same. This implies that we no longer have the advantageous property of being able to tune the controller for tracking performance by perturbing only the initial conditions of the servocompensator $\eta_{0}$ while minimizing the performance index $J_{B}$ (2.70), as already described in Section 2.5.1. This is another reason for choosing controller (2.6) in addition to the properties of the controller discussed in Section 2.2.1. 


\subsection{Experimental Implementation of Proposed Con- troller Design}

The evaluation of the performance index $J_{B \eta_{0}}(2.79)$

$$
J_{B \eta_{0}}=h \times \operatorname{trace}\left(\left[\begin{array}{ll}
0 & I
\end{array}\right] \overline{\mathcal{P}}\left[\begin{array}{l}
0 \\
I
\end{array}\right]\right)
$$

where $\overline{\mathcal{P}}$ is the solution to the discrete Lyapunov equation in (2.76), requires either a knowledge of the plant model or an infinite number of experiments to be performed in order to measure the "average cost" over all initial conditions $\eta_{0}$ uniformly distributed on a unit disk ball. Instead, we propose to take the approach of evaluating the performance index $J_{B}(2.70)$

$$
J_{B}=h \sum_{k=0}^{\infty}\left(\Delta z_{k}\right)^{\top}\left(\Delta z_{k}\right)+\mu\left(\Delta u_{k}\right)^{\top}\left(\Delta u_{k}\right)
$$

where $\Delta z_{k}=\theta e_{k}+\left(\eta_{k}-\eta_{\infty}\right)$ and $\Delta u_{k}=u_{k}-u_{\infty}$, using only the measurable signals $e_{k}, \eta_{k}, u_{k}, \eta_{\infty}$, and $u_{\infty}$ for a finite number of points uniformly distributed on the unit disk ball. Each point on the unit ball corresponds to carrying out an experiment. This approach provides an approximation to the performance index $J_{B \eta_{0}}$ and the approximation improves as we increase the number of points on the unit ball. It should be noted that we need to consider only half of the unit disk ball when choosing the finite number of points due to symmetry. We will denote the chosen number of points on the unit ball by the variable $q$ and Figure 2.1 illustrates the approach with $q=5$. For example, assume the controller is $u_{k}=-2 y_{k}-0.5 \eta_{k}$ and carry out an experiment with $\eta_{0}=\left[\begin{array}{ll}0 & 1\end{array}\right]^{\top}$ to obtain the first cost value $J_{1}$. We then repeat the experiment with $\eta_{0}=\left[\begin{array}{ll}1 / \sqrt{2} & 1 / \sqrt{2}\end{array}\right]^{\top}$ to obtain the second cost $J_{2}$. A total of 5 experiments is performed (each time with a different $\eta_{0}$ ) and an average value of the respective costs is then obtained. This average value represents the final cost for this specific controller (with gains $K_{P}=2$ and $\left.K_{I}=0.5\right)$ and is used as one data point in the controller parameter optimization. For convenience, we will denote the points chosen on the unit ball by a set denoted by $\mathcal{Z}$. For the example presented above we have

$$
\mathcal{Z}_{\eta_{0}}=\left\{\left[\begin{array}{l}
0 \\
1
\end{array}\right],\left[\begin{array}{c}
\frac{1}{\sqrt{2}} \\
\frac{1}{\sqrt{2}}
\end{array}\right],\left[\begin{array}{l}
1 \\
0
\end{array}\right],\left[\begin{array}{c}
\frac{1}{\sqrt{2}} \\
-\frac{1}{\sqrt{2}}
\end{array}\right],\left[\begin{array}{c}
0 \\
-1
\end{array}\right]\right\}
$$

where there are $q$ number of elements and the subscript $\eta_{0}$ indicates the variable of interest. The experimental approach described can also be adopted for measuring the 
average cost over $y_{\text {ref }}$ or $\omega$ signals that are uniformly distributed on the unit ball. In general, $x_{0}$ can be assumed to be 0 if one lets the system settle to its equilibrium point before carrying out the experiments.

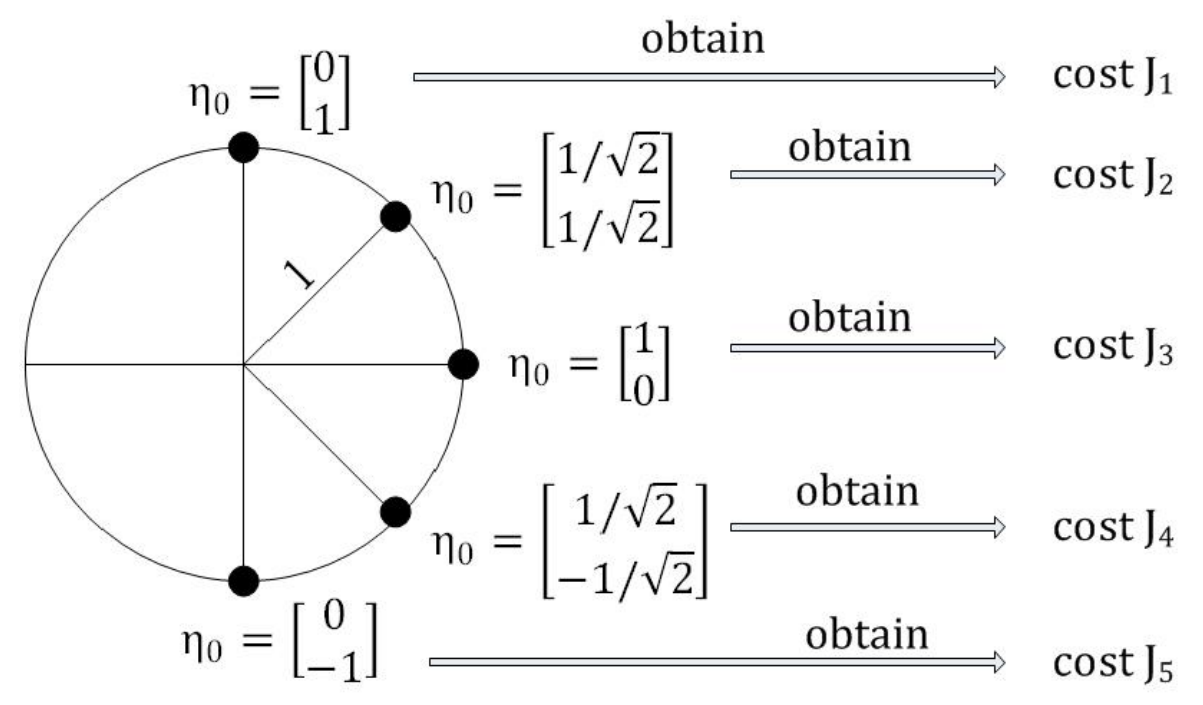

Figure 2.1: A selection of $\eta_{0}$ values for calculating the average cost.

\subsubsection{Performance Index Modification}

To assure that the resultant closed-loop system remains stable during the experimental parameter optimization, the following modified performance indices are used:

$$
J_{A}= \begin{cases}\frac{1}{h} \sum_{k=1}^{T}\left(\Delta z_{k}\right)^{\top}\left(\Delta z_{k}\right)+\mu\left(\Delta u_{k}\right)^{\top}\left(\Delta u_{k}\right), & \text { if actuators do not saturate } \\ 10^{20}, & \text { otherwise }\end{cases}
$$

where $J_{A}$ is given by either $(2.56),(2.57),(2.58),(2.59)$, and where $\Delta z_{k}=\theta\left(e_{k}-e_{k-1}\right)$ $+h e_{k-1}$ and $\Delta u_{k}=u_{k}-u_{k-1}$. Likewise,

$$
J_{B}= \begin{cases}h \sum_{k=0}^{T}\left(\Delta z_{k}\right)^{\top}\left(\Delta z_{k}\right)+\mu\left(\Delta u_{k}\right)^{\top}\left(\Delta u_{k}\right), & \text { if actuators do not saturate } \\ 10^{20}, & \text { otherwise }\end{cases}
$$

where $J_{B}$ is given by either $(2.78),(2.79),(2.80),(2.81)$, and where $\Delta z_{k}=\theta e_{k}+\left(\eta_{k}-\eta_{\infty}\right)$ and $\Delta u_{k}=u_{k}-u_{\infty}$. Here the measure of instability used is that if the control signal saturates it is assumed that the closed-loop system is unstable. The duration of each experiment is chosen to be long enough such that both transient and steady state responses 
are captured, i.e., $T$ is a sufficiently large number to approximate $\infty$. When actuator saturation occurs, due to system instability, a large value such as $10^{20}$ can be assigned to the performance index value $J$. This will force the optimization algorithm to converge to a set of controller gains that will maintain closed-loop stability and will not cause the actuators to saturate. This implies that the parameter optimization algorithm must not fail on discontinuous or non-differentiable functions (see Section 3.1 for the choice of algorithm). It is also desired to avoid actuators from saturating since a non-linear phenomenon known as reset windup [5] will occur if a controller with integral action is used which saturates. When this happens the actuator will remain at its limit independently of the plant's output and the error signal will continue to be integrated. This means that the integral term may become very large and will require the error signal to have an opposite sign for a long period to "undo" the integration. The consequence is that large transients may arise when the actuator saturates.

\subsubsection{Controller Implementation}

When implementing the discrete controller (2.6), the sampling period $h$ must be chosen to be greater than the time $h_{c}$ required to execute all of the following instructions during each sampling period:

1. read input port,

2. compute control signal,

3. set output port, and

4. update controller variables.

In view of the real-time scheduling constraints, the controller (2.6) can be implemented as

$$
\begin{aligned}
u_{t} & =-S\left(K_{P} y_{t-h_{c}}+K_{I} \eta_{t-h_{c}}\right) \\
\eta_{t} & =\eta_{t-h_{c}}+h e_{t-h_{c}} \\
& =\eta_{t-h_{c}}+h\left(y_{t-h_{c}}-y_{r e f}\right),
\end{aligned}
$$

which is an "approximation" to the continuous controller

$$
\begin{aligned}
& u(t)=-S\left(K_{P} y(t)+K_{I} \eta(t)\right) \\
& \dot{\eta}(t)=e(t) .
\end{aligned}
$$


The time sequence for controller (2.104) is shown in Figure 2.2.

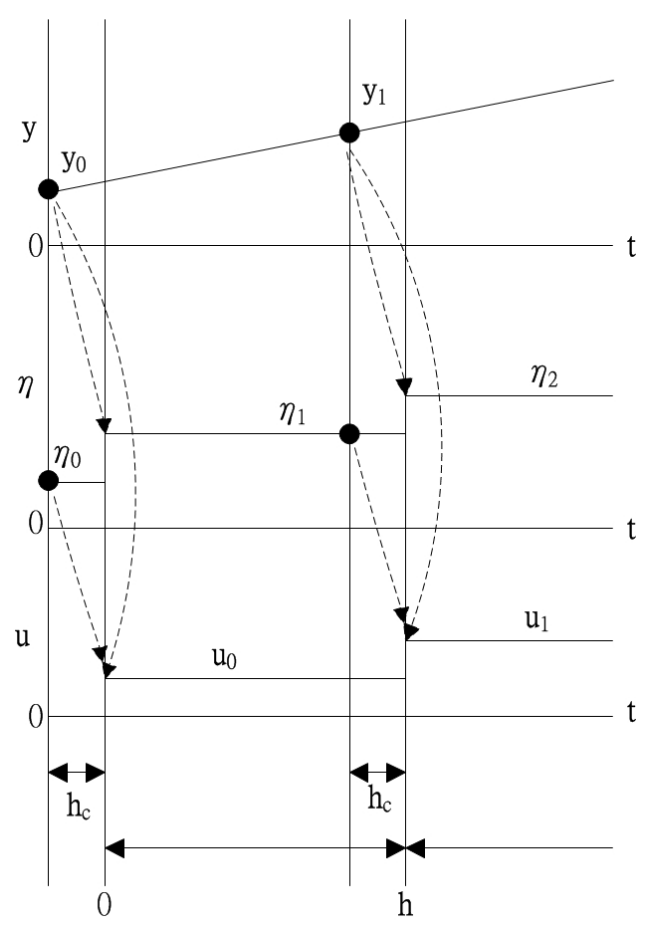

Figure 2.2: The time sequence for the two-term controller.

\subsection{Optimization Procedure}

The controller parameter optimization process can be summarized into the following steps:

1. Choose a performance index either (2.56), (2.57), (2.58), (2.59), (2.78), (2.79), (2.80), or (2.81) depending on the nature of the controller design specifications.

2. Measure the steady-state tracking matrix $\Gamma$ to determine if a controller exists that solves the robust servomechanism problem.

3. Select a set of $y_{0}, \eta_{0}, \omega$ or $y_{r e f}$ signals that are uniformly distributed on a scaled unit disk ball such that the steady-state value of the $i^{t h}$ control input is within the saturation limits of the $i^{t h}$ actuator, i.e., $\left|u_{\infty}(i)\right| \leq\left|u_{\text {Sat }}(i)\right|, \quad i=1,2, \ldots, m$. According to (2.16), $u_{\infty}$ can be expressed as $u_{\infty}=\Gamma^{-1} y_{r e f}-\Gamma^{-1} \Lambda \omega$. Both the $\Gamma$ and $\Lambda$ matrices can be obtained experimentally using the procedure outlined in Section 2.2.2 to achieve this. 
4. Precondition the plant with the tuning regulator $u_{k}=-\epsilon S \eta_{k}$, where $\epsilon$ is sufficiently small.

5. Carry out experiments to minimize the performance index $J_{A}(2.102)$ or $J_{B}(2.103)$. Start with a large control penalty coefficient $\mu$ in the performance index and choose sufficiently small $K_{P}$ and $K_{I}$ values as feasible starting gains for the optimization problem.

6. Decrease the $\mu$ value and repeat the optimization problem, using the optimal controller gains obtained in step 5 as a new starting point.

7. If a desired closed-loop response is achieved, stop.

If a desired closed-loop response is not achieved, this may or may not be due to the plant having nonminimum phase effects. Alternately, it may be due to the simplicity of the two-term controller being used, and so one could repeat the design process using a more complex controller.

Pre-conditioning the system with the tuning regulator in step 3 guarantees a nonsaturating solution to the robust servomechanism problem. Recall from Section 2.3 that the tuning regulator has the property of achieving closed-loop stability without violating the saturation constraints. The addition of the controller $u_{k}=-S\left(K_{P} y_{k}+K_{I} \eta_{k}\right)$ is solely to improve the system response. If $\mu$ is large, the performance index or cost function is dominated by the control effort $u_{k}$. In the case of a stable plant, solving the optimization problem with $\mu \rightarrow \infty$ will result in $\left(K_{P}, K_{I}\right) \rightarrow(0,0)$. Thus, a nonsaturating solution to RSP is obtained, which is just the tuning regulator itself.

We will now apply the proposed controller design approach to 4 different classes of systems:

- Open-loop stable and minimum phase MIMO small-scale system (see Chapter 4).

- Open-loop stable and minimum phase MIMO large-scale system (see Chapter 5).

- Open-loop stable and nonminimum phase MIMO medium-scale system (see Chapter 6).

- Open-loop stable SISO system with time delay (see Chapter 7). 


\section{Chapter 3}

\section{Optimization Algorithm and Simulation Procedure}

In this chapter, the mechanics and properties of the optimization algorithm that we chose to solve the two optimization problems A and B, are discussed. The properties of the algorithm are further elaborated on through comparisons made against gradient-based algorithms and other popular direct search methods. A procedure for carrying out the proposed controller design method in simulation is also provided.

\subsection{The Chosen Algorithm}

The optimization problems A and B will be solved using the Nelder-Mead algorithm [33], which is a direct search method for the minimization of a function of $n$ variables. The algorithm operates by first comparing the function values at the $(n+1)$ vertices of a simplex in a $n$ dimensional parameter space, followed by the replacement of the vertex with the highest function value by another point obtained via one of the four operations at each iteration - reflection, contraction, expansion and shrinkage.

Assume a $n$ dimensional simplex with vertices $v_{0}, v_{1}, \ldots, v_{n}$, and let $f_{i}$ be the function value at $v_{i}$. Further, define $v_{l}$ to be the vertex with lowest function value $f_{l}$; $v_{h}$ to be the vertex with highest function value $f_{h}$; and $\bar{v}$ to be the centroid of the remaining $n$ vertices. The reflection of $v_{h}$ over $\bar{v}$ along the line joining $v_{h}$ and $\bar{v}$ is a new vertex $v^{*}$, whose coordinates are defined by the relation

$$
v^{*}=\bar{v}+\alpha\left(\bar{v}-v_{h}\right)
$$


where $\alpha>0$ is the positive reflection coefficient. If $y_{h}>y^{*}>y_{l}$, then a new working simplex is formed by replacing $v_{h}$ with $v^{*}$. If $y^{*}<y_{l}$, then the simplex is further expanded along the reflection line to a new vertex $v^{* *}$ by the relation

$$
v^{* *}=\bar{v}+\gamma\left(v^{*}-\bar{v}\right)
$$

where $\gamma>1$ is the expansion coefficient. If $y^{* *}<y_{l}, v_{h}$ is replaced by $v^{* *}$; otherwise, $v_{h}$ is replaced by $v^{*}$.

If the initial reflection was unsuccessful, i.e., $y^{*}>y_{i}$ for all $i \neq h$, then $v_{h}$ remains unchanged or is set to $v_{h}=v^{*}$ depending on which has a lower function value. A contracted point $v^{* *}$ is then formed by the relation

$$
v^{* *}=\bar{v}+\beta\left(v_{h}-\bar{v}\right)
$$

where $0<\beta<1$ is the contraction coefficient and set $v_{h}=v^{* *}$. However, if the contraction fails to give rise to a vertex with a lower function value than $y_{h}$, the current simplex is shrunk by replacing all the $v_{i}$ by $\left(v_{i}+v_{l}\right) / 2$.

In setting up the algorithm, we choose to set $\alpha=1, \beta=0.5, \gamma=2$ and let the iterations continue until the length of the longest edge of the simplex becomes smaller than some tolerance value. The mechanics of the Nelder-Mead algorithm described above can be visualized as seen in Figures 3.1 and 3.2.

The Nelder-Mead method has some advantages and disadvantages:

Advantages

- The method does not involve approximating derivatives and thus is "robust," i.e., it does not fail on discontinuous or non-differentiable functions. This allows us to impose stability and saturation constraints for solving the controller parameter optimization problem by modifying the function to take large positive values for controller gains that result in closed-loop instability or actuator saturation.

- The method has the ability to adapt itself to "local landscape" such that every trespassing by the simplex over constraint borders will be followed by contraction moves which force the simplex to remain in the feasible region [33].

- The method tends to give significant improvements in reducing the function value in the first few iterations. This is appealing in many practical problems such as process control, where each function evaluation can be time consuming and expensive. 


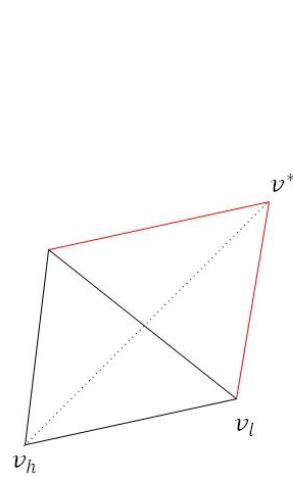

(a)

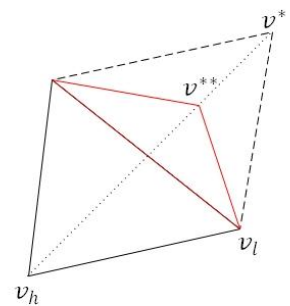

(c)

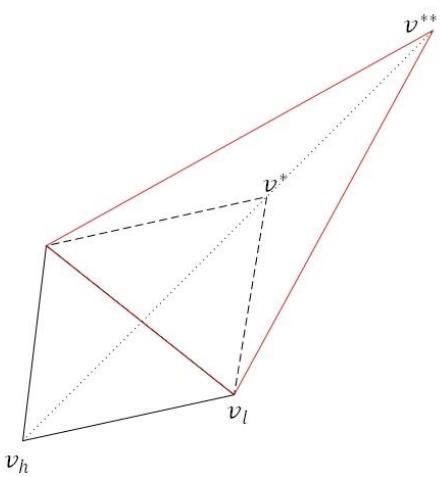

(b)

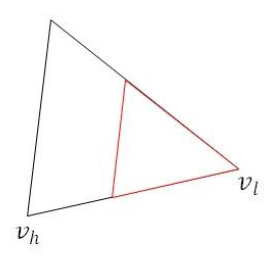

(d)

Figure 3.1: Possible transformations of the simplex: (a) reflection, (b) expansion, (c) contraction, and (d) shrinkage [34].

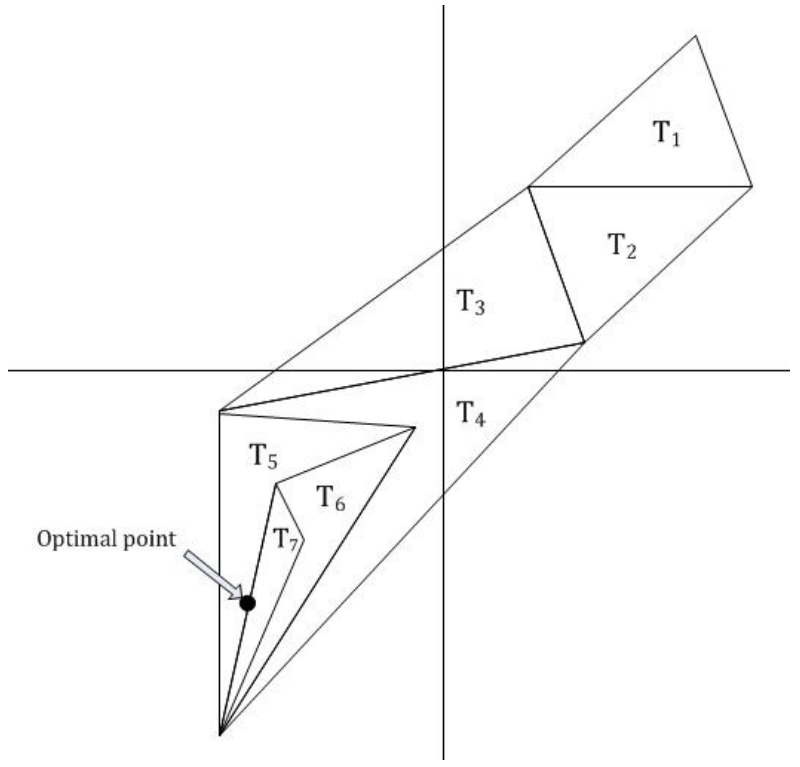

Figure 3.2: The sequence of triangles $\left\{T_{k}\right\}$ converging to the optimal point for the NelderMead algorithm [34]. 


\section{Disadvantages}

- The method can quickly approach the vicinity of the optimal point, but may be slow in locating the minimum itself, thus, requiring many more function evaluations as compared to gradient based methods.

- There are essentially no theoretical results or proofs on the convergence properties of the algorithm, except for the case of a one dimensional parameter space [35]. It may only give local solutions. As a result, one may need to try different starting points, different sizes or orientations of the initial simplex and/or repeat the algorithm multiple times before reaching the global minimizer.

The first motivation for using the Nelder-Mead method was to recognize that the performance index to be minimized may not be differentiable, which implies that gradientbased methods such as Quasi-Newton or Conjugate Gradient methods may have numerical problems. This situation can arise when a penalty function is used when the closed-loop eigenvalues are unstable. For example, penalty functions in the form of

$$
F(x)= \begin{cases}f(x) & \text { if } x \in \Omega \\ +\infty & \text { otherwise }\end{cases}
$$

or

$$
F(x)=f(x)+\sum_{i=1}^{m} \omega_{i} \max \left(0, c_{i}(x)\right)
$$

where $f(x)$ is the objective function, $c(x)$ is the constraint function, $\Omega$ is the feasible set and the weights $\omega_{i}>0$ are chosen to be sufficiently large, clearly leads to a nondifferentiable performance index.

The issues associated with gradient-based methods naturally lead to the investigation of heuristic methods or so called Direct Search methods. Some of the popular algorithms in this category includes Nelder-Mead, Pattern Search, and the Genetic Algorithm. Pattern search, in short, is a method where the the points at which the function is evaluated form a grid of points in $\mathbb{R}^{n}$. A grid is formed around the current iterate by selecting points that radiate outwards from this center point according to a set of specified directional vectors. In general, the grid is composed of at least $2 n$ points, and the emphasis is on keeping the points close to the current iterate as to aquire a detailed local picture of the objective function before making a decision about where to move [36]. This means that all points in the grid are evaluated at each iteration. If the initial starting point is 
far from the optimal point, much time is wasted on function evaluations that may not be necessary, where as Nelder-Mead performs only one or two function evaluations per iteration regardless of the size of $n$, except in shrink transformations, which are rare in practice [37].

The Genetic Algorithm is a method that simulates biological evolution to solve optimization problems. The algorithm starts out with a random initial population and creates a sequence of new generations, through random biological selections, crossovers, and mutations at each iteration, that evolve towards an optimal solution [38]. Similar to the concerns for Pattern Search, Genetic Algorithm involves a large number of function evaluations per iteration and can be computationally intensive. As a result of all the discussions thus far, we have chosen the Nelder-Mead algorithm for our applications.

\subsection{Illustration Example}

Consider an unstable SISO plant given by

$$
G(s)=\frac{s^{2}+3 s+2}{s^{2}-8 s+15}
$$

where the poles are located at $s=3$ and $s=5$. A proportional controller is to be designed to stabilize the plant as shown in Figure 3.3.

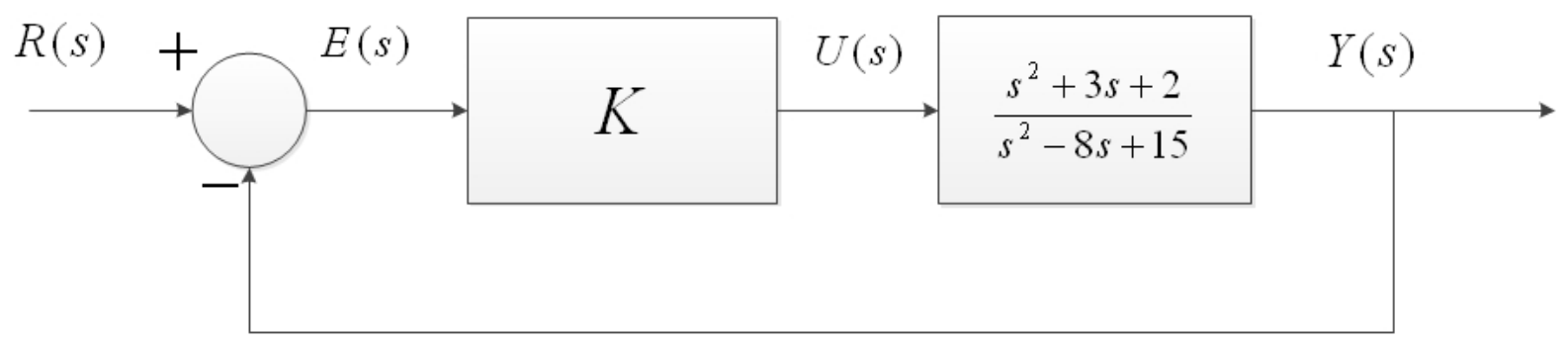

Figure 3.3: Block diagram of the feedback control system.

From the root locus plot shown in Figure 3.4, the plant can indeed be stabilized by some controller gain $K$. We propose that $K$ is to be chosen by solving the following unconstrained optimization problem:

$$
\underset{K}{\operatorname{minimize} \max }(\Re\{\sigma(G(s))\})
$$

where $\sigma(\cdot)$ is the pole spectrum of the argument. The objective function (3.7) can be interpreted as minimizing the real component of the largest closed-loop pole, and is nondifferentiable at where the break-in and breakaway occurs in the root locus diagram. 
According to the root locus diagram, the optimal gain is $K=116$ and the largest pole will be located at $s=-1.45$.

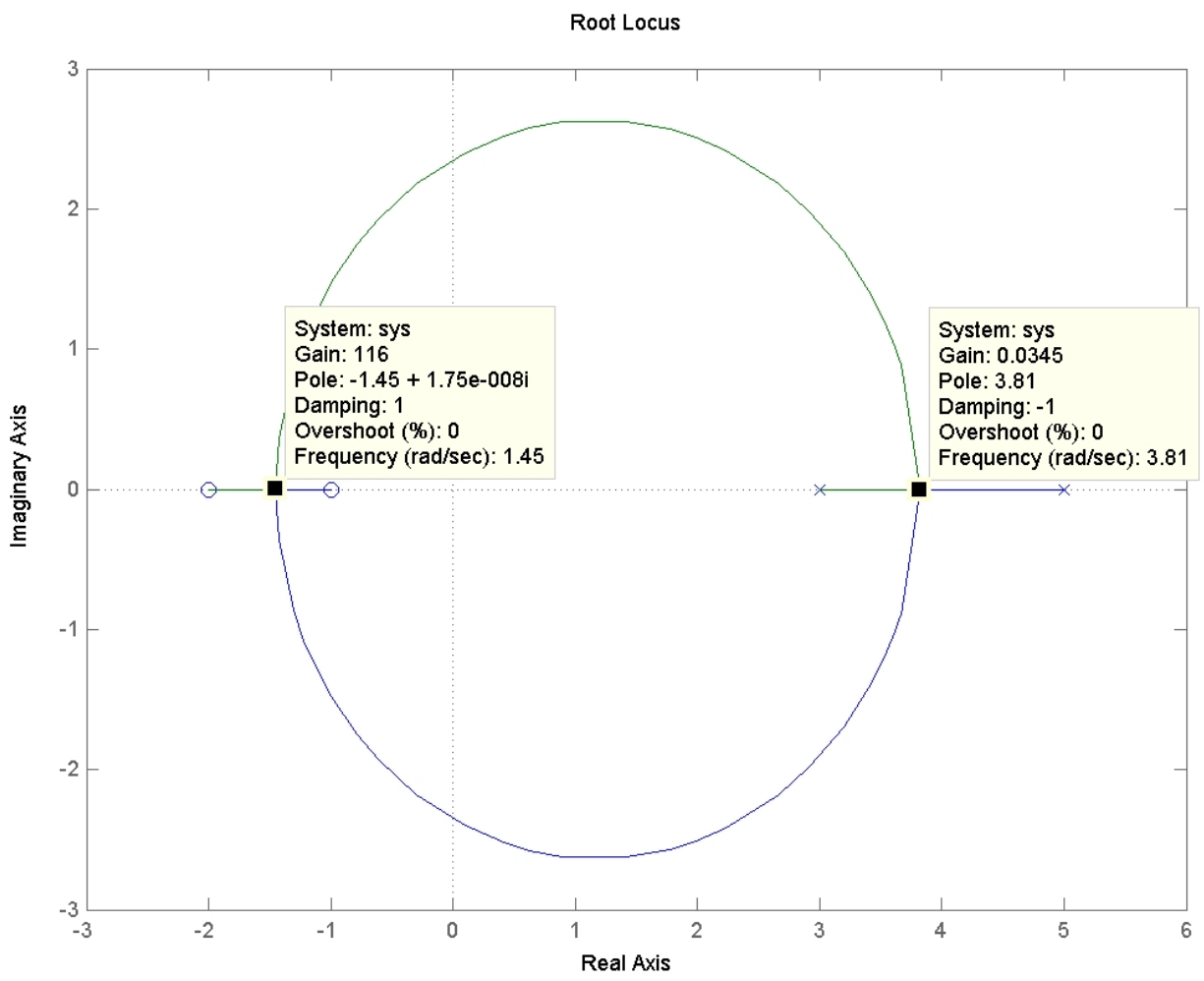

Figure 3.4: Root locus of the feedback control system.

By applying the Nelder-Mead algorithm, the following optimization output is obtained for the initial starting point of $K=10^{-3}$ :

\begin{tabular}{|cccl|}
\hline Iteration & Func-count & $\min f(\mathrm{x})$ & Procedure \\
0 & 1 & 4.97891 & \\
1 & 2 & 4.97786 & initial simplex \\
2 & 4 & 4.97574 & expand \\
3 & 6 & 4.97149 & expand \\
4 & 8 & 4.96298 & expand \\
5 & 10 & 4.94588 & expand \\
6 & 12 & 4.91129 & expand \\
7 & 14 & 4.84045 & expand \\
8 & 16 & 4.69032 & expand \\
9 & 18 & 4.32516 & expand \\
10 & 20 & 3.72739 & expand \\
11 & 22 & 3.48482 & expand \\
12 & 24 & 3.06148 & expand \\
13 & 26 & 2.39919 & expand
\end{tabular}




\begin{tabular}{|c|c|c|c|}
\hline 14 & 28 & 1.52173 & expand \\
\hline 15 & 30 & 0.583846 & expand \\
\hline 16 & 32 & -0.214277 & expand \\
\hline 17 & 34 & -0.771962 & expand \\
\hline 18 & 36 & -1.11015 & expand \\
\hline 19 & 38 & -1.29791 & expand \\
\hline 20 & 40 & -1.39706 & expand \\
\hline 21 & 42 & -1.44804 & expand \\
\hline 22 & 44 & -1.44804 & contract inside \\
\hline 23 & 46 & -1.44804 & contract inside \\
\hline 24 & 48 & -1.44804 & contract inside \\
\hline 25 & 50 & -1.45107 & reflect \\
\hline 26 & 52 & -1.45107 & contract inside \\
\hline 27 & 54 & -1.45246 & reflect \\
\hline 28 & 56 & -1.45246 & contract inside \\
\hline 29 & 58 & -1.45246 & contract inside \\
\hline 30 & 60 & -1.45279 & reflect \\
\hline 31 & 62 & -1.45279 & contract inside \\
\hline 32 & 64 & -1.45296 & reflect \\
\hline 33 & 66 & -1.45296 & contract inside \\
\hline 34 & 68 & -1.45296 & contract inside \\
\hline 35 & 70 & -1.45296 & contract inside \\
\hline 36 & 72 & -1.45296 & contract inside \\
\hline 37 & 74 & -1.45297 & reflect \\
\hline 38 & 76 & -1.45297 & contract inside \\
\hline 39 & 78 & -1.45297 & reflect \\
\hline 40 & 80 & -1.45297 & contract inside \\
\hline 41 & 82 & -1.45298 & reflect \\
\hline 42 & 84 & -1.45298 & contract inside \\
\hline 43 & 86 & -1.45298 & contract inside \\
\hline 44 & 88 & -1.45298 & reflect \\
\hline 45 & 90 & -1.45298 & contract inside \\
\hline 46 & 92 & -1.45298 & reflect \\
\hline 47 & 94 & -1.45298 & contract inside \\
\hline 48 & 96 & -1.45298 & reflect \\
\hline 49 & 98 & -1.45298 & contract inside \\
\hline 50 & 100 & -1.45298 & contract inside \\
\hline \multicolumn{4}{|c|}{$\begin{array}{l}\text { Optimization terminated: } \\
\text { the current } x \text { satisfies the termination criteria using } \\
\text { OPTIONS.TolX of } 1.000000 e-004 \text { and } F(X) \text { satisfies the } \\
\text { convergence criteria using OPTIONS.Tolfun of } 1.000000 \text {-0004 }\end{array}$} \\
\hline \multicolumn{4}{|c|}{ optimal gain is $K=115.9654$} \\
\hline
\end{tabular}

The results show that the expected optimal gain and pole locations are obtained, but it also shows that the algorithm is slow in locating the minimum itself. 


\subsection{Description of the Simulation Procedure}

The controller design process can be carried out in simulation, using a combination of MATLAB and Simulink, where the Simulink model simulates the closed-loop system response for generating input and output data and the MATLAB environment executes the Nelder-Mead algorithm. The process consists of the following steps:

1. Run the code for the Nelder-Mead algorithm in the MATLAB environment and provide a starting point for the algorithm.

2. Simulate the closed-loop response in Simulink whenever the performance index of a controller with specified gains needs to be evaluated. Meanwhile, the Nelder-Mead algorithm is paused in the background.

3. Calculate the performance index value or cost using the input and output data signals that are logged into the MATLAB workspace at the end of the current simulation run. If control input saturation occurs, the cost is assigned a value of $10^{20}$.

4. Store the cost and resume the execution of the algorithm to determine a new set of controller gains to evaluate.

5. Repeat steps 2 to 4 until the algorithm converges. 


\section{Chapter 4}

\section{MARTS Simulation Example (Minimum Phase System)}

\subsection{Description of MARTS}

The MARTS (Multivariable Apparatus for Real Time Control Studies) in [39] is a two vessel hydraulic system model with 2 inputs, 2 outputs, and 2 disturbance input channels. A schematic of MARTS is shown in Figure 4.1 with the inputs $u$ and outputs $y$ measured in volts. A $1 V$ increase/decrease at the output represents approximately $2.4 L$ of water rise/drop or a $30 \mathrm{~cm}$ change in water level height. A $1 V$ change in the input to control valve 1 and 2 corresponds to a change in water flow rate of approximately $0.0072 L / s$ and $0.0108 \mathrm{~L} / \mathrm{s}$ respectively.

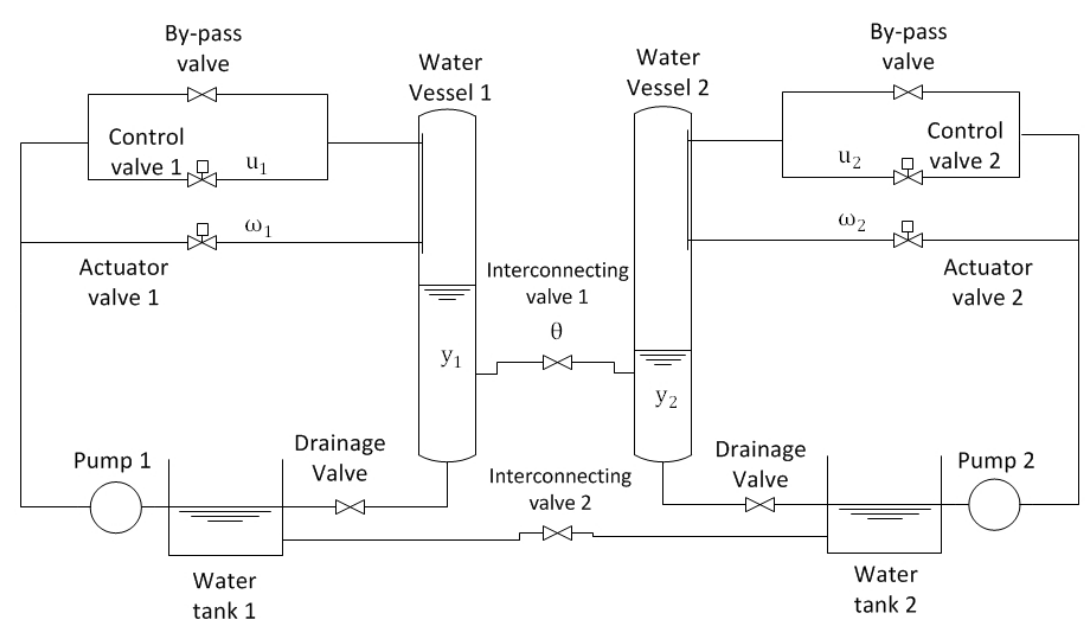

Figure 4.1: Schematic of MARTS setup. 
By applying the identification methods given in [39] for the case when the interconnecting valve 1 is set to $\theta \approx 30^{\circ}$ and the disturbance signals $\omega$ disabled, the following approximate continuous linear time-invariant model is obtained:

$$
\begin{aligned}
\dot{x}(t) & =\mathcal{A} x(t)+\mathcal{B} u(t) \\
y(t) & =\mathcal{C} x(t)+\mathcal{D} u(t)
\end{aligned}
$$

where $\mathcal{A}, \mathcal{B}, \mathcal{C}$, and $\mathcal{D}$ are given by

$$
\begin{aligned}
& \mathcal{A}=\left[\begin{array}{cc}
-6.18 \times 10^{-2} & 1.35 \times 10^{-2} \\
1.35 \times 10^{-2} & -8.00 \times 10^{-2}
\end{array}\right] \\
& \mathcal{B}=\left[\begin{array}{cc}
3.00 \times 10^{-3} & 0 \\
0 & 4.50 \times 10^{-3}
\end{array}\right] \\
& \mathcal{C}=\left[\begin{array}{ll}
1 & 0 \\
0 & 1
\end{array}\right] \\
& \mathcal{D}=\left[\begin{array}{ll}
0 & 0 \\
0 & 0
\end{array}\right] .
\end{aligned}
$$

The eigenvalues of this continuous system are given by

$$
\left\{-8.7181 \times 10^{-2},-5.4619 \times 10^{-2}\right\}
$$

and there are no transmission zeros. If we sample the system with $h=0.1$, the resultant sampled system is open-loop stable and minimum phase with no transmission zeros, and with eigenvalues given by

$$
\left\{9.9945 \times 10^{-1}, 9.9913 \times 10^{-1}\right\}
$$

It is to be noted that the mathematical model of the plant (4.1) is used in this chapter only to carry out simulations, but it is not used for controller design.

The operating range of the control signals is given by

$$
\begin{aligned}
& -5 V \leq u_{k}(1) \leq 5 V \\
& -5 V \leq u_{k}(2) \leq 5 V .
\end{aligned}
$$

Open-loop responses of this system are given in Figure 4.2. The steady-state tracking matrix $\Gamma$ (2.2) is experimentally obtained to be:

$$
\Gamma=\left[\begin{array}{ll}
5.04 \times 10^{-2} & 1.28 \times 10^{-2} \\
8.51 \times 10^{-3} & 5.84 \times 10^{-2}
\end{array}\right]
$$



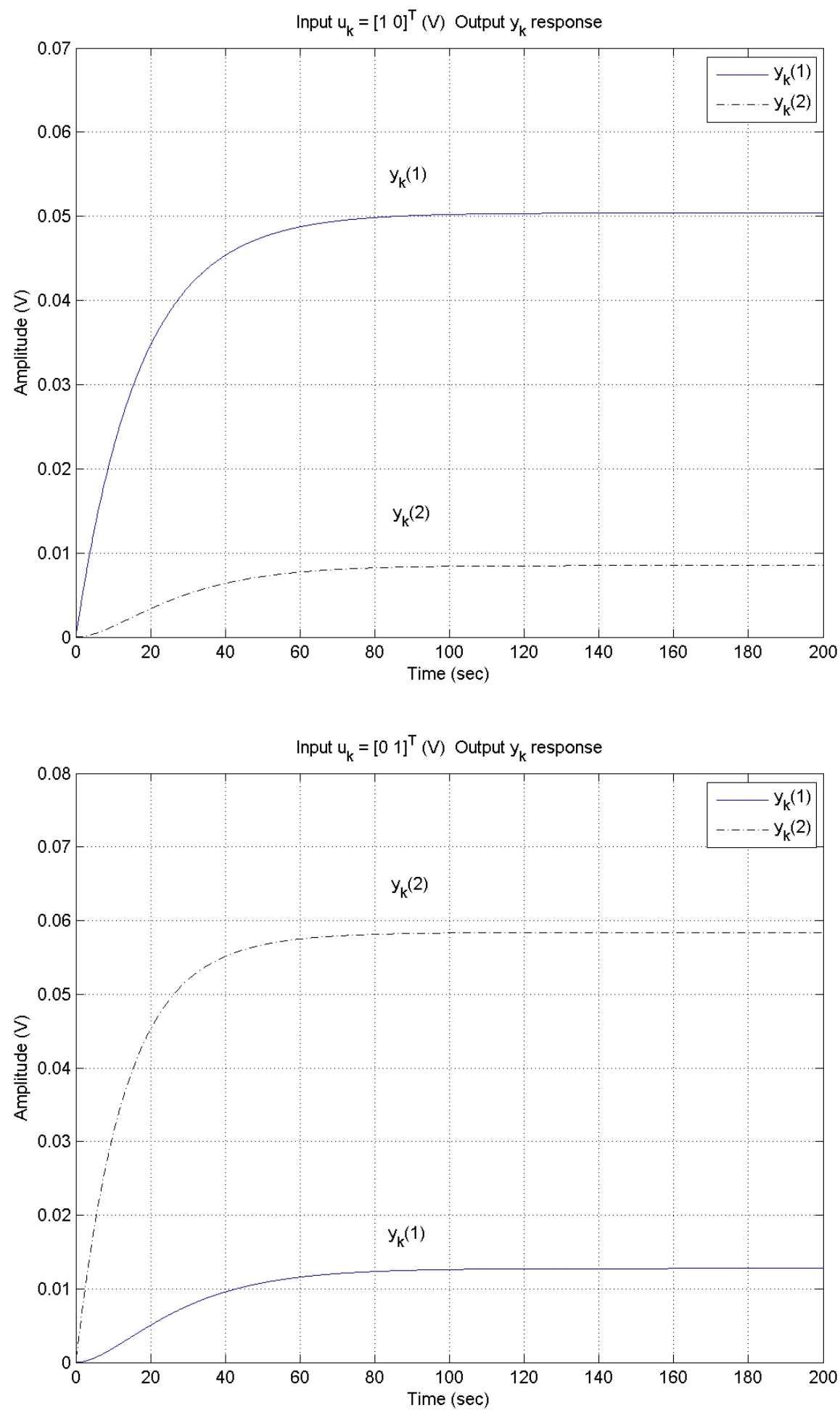

Figure 4.2: MARTS Example, Open-Loop Response. 


\subsection{Control Design 1 for MARTS}

In this study, it is desired to design a controller to provide tracking for constant tracking signals $y_{r e f}(1), y_{r e f}(2)$. The tuning regulator (2.17) with $\epsilon=10^{-3}$ is first applied to the plant and the two-term controller (2.6) is then obtained by minimizing the performance index $J_{A y_{\text {ref }}}(2.59)$ using $\theta=0, \mu=10^{3}$. The optimization is carried out under the system settings of:

$$
x_{0}=\left[\begin{array}{l}
0 \\
0
\end{array}\right], \eta_{0}=\left[\begin{array}{l}
0 \\
0
\end{array}\right], \text { and } \mathcal{Z}_{y_{r e f}}=\left\{\left[\begin{array}{c}
0 \\
0.2
\end{array}\right],\left[\begin{array}{c}
\frac{0.2}{\sqrt{2}} \\
\frac{0.2}{\sqrt{2}}
\end{array}\right],\left[\begin{array}{c}
0.2 \\
0
\end{array}\right],\left[\begin{array}{c}
\frac{0.2}{\sqrt{2}} \\
-\frac{0.2}{\sqrt{2}}
\end{array}\right]\right\}
$$

The above settings correspond to approximating the performance index $J_{A y_{\text {ref }}}(2.59)$; in this case the optimal control gains $K_{P}$ and $K_{I}$ are found to be

$$
K_{P}=3.4475 \times 10^{-2}, \quad K_{I}=9.3686 \times 10^{-4} .
$$

Simulation of the tracking reference input is given in Figures 4.3-1 to 4.3-4 for the resulting closed-loop system.

Remark 4.1. Although the states $x_{k}$ can not be measured, we allow the system to settle to its equilibrium point before performing each experiment; hence, $x_{0}$ can be assumed to be 0 .

Remark 4.2. The $y_{\text {ref }}$ signals are chosen from a disk of radius 0.2 . A simple calculation of $u_{\infty}=\Gamma^{-1} y_{\text {ref }}$ shows that the steady state control input is within the actuator saturation bounds for the chosen set of tracking signals $y_{r e f}$.

Remark 4.3. With the chosen $y_{\text {ref }}$ signals, the steady-state control input $u_{\infty}=4.12 \mathrm{~V}$ is close to the saturation bounds $|u|<5 \mathrm{~V}$. It is seen that the closed-loop system using the tuning regulator is unsaturated, where the control input $u_{k}$ monotonically approaches to $u_{\infty}$ with no overshoot. A large control penalty coefficient $\mu$ is chosen to ensure that the addition of the tuned two-term controller does not cause saturation during the optimization process. 

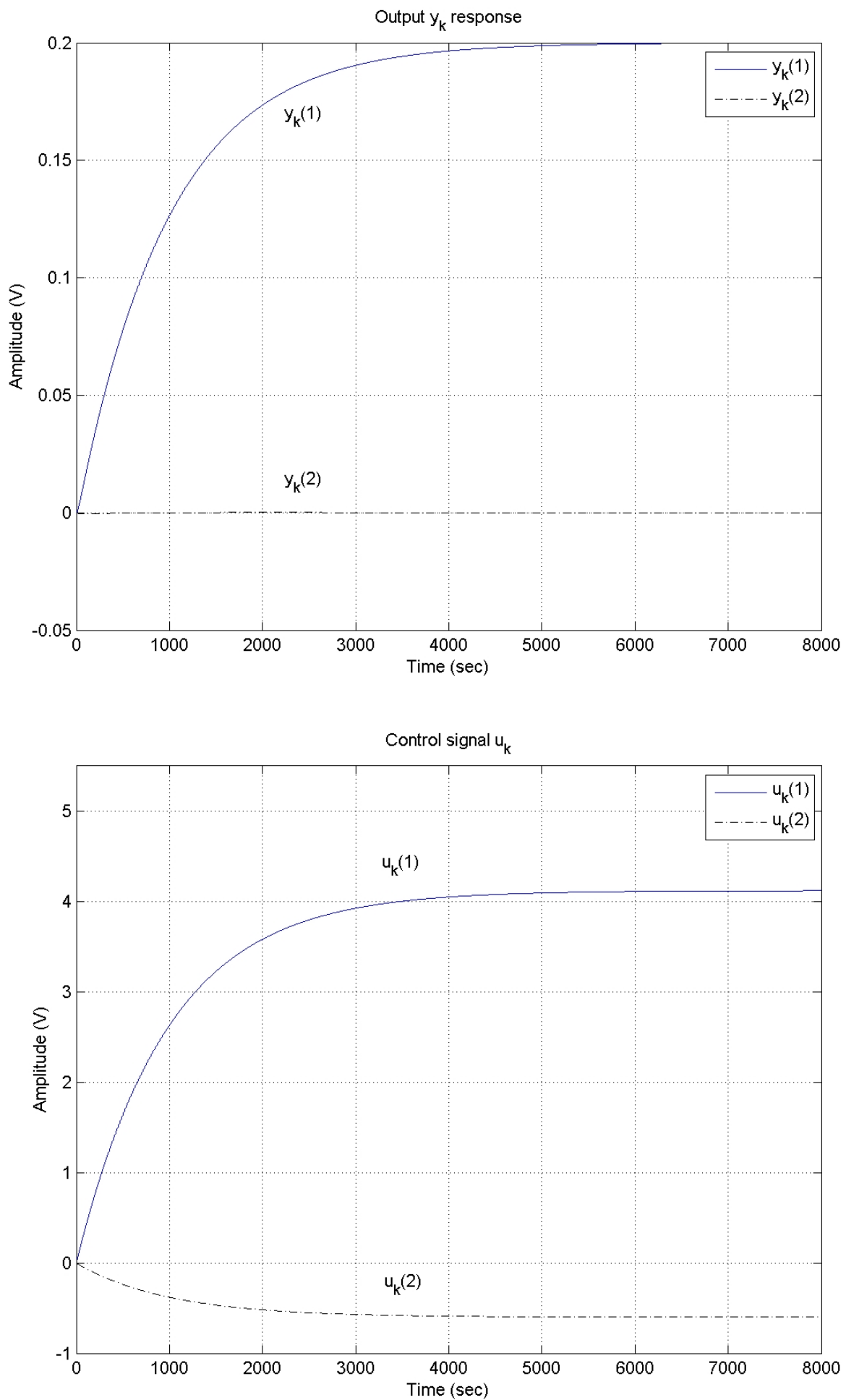

Figure 4.3-1: MARTS Example, Closed-Loop Response Tuning Regulator (2.17) with $\epsilon=10^{-3}$ Input $y_{\text {ref }}=\left[\begin{array}{ll}0.2 & 0\end{array}\right]^{\top}$ Design 1. 

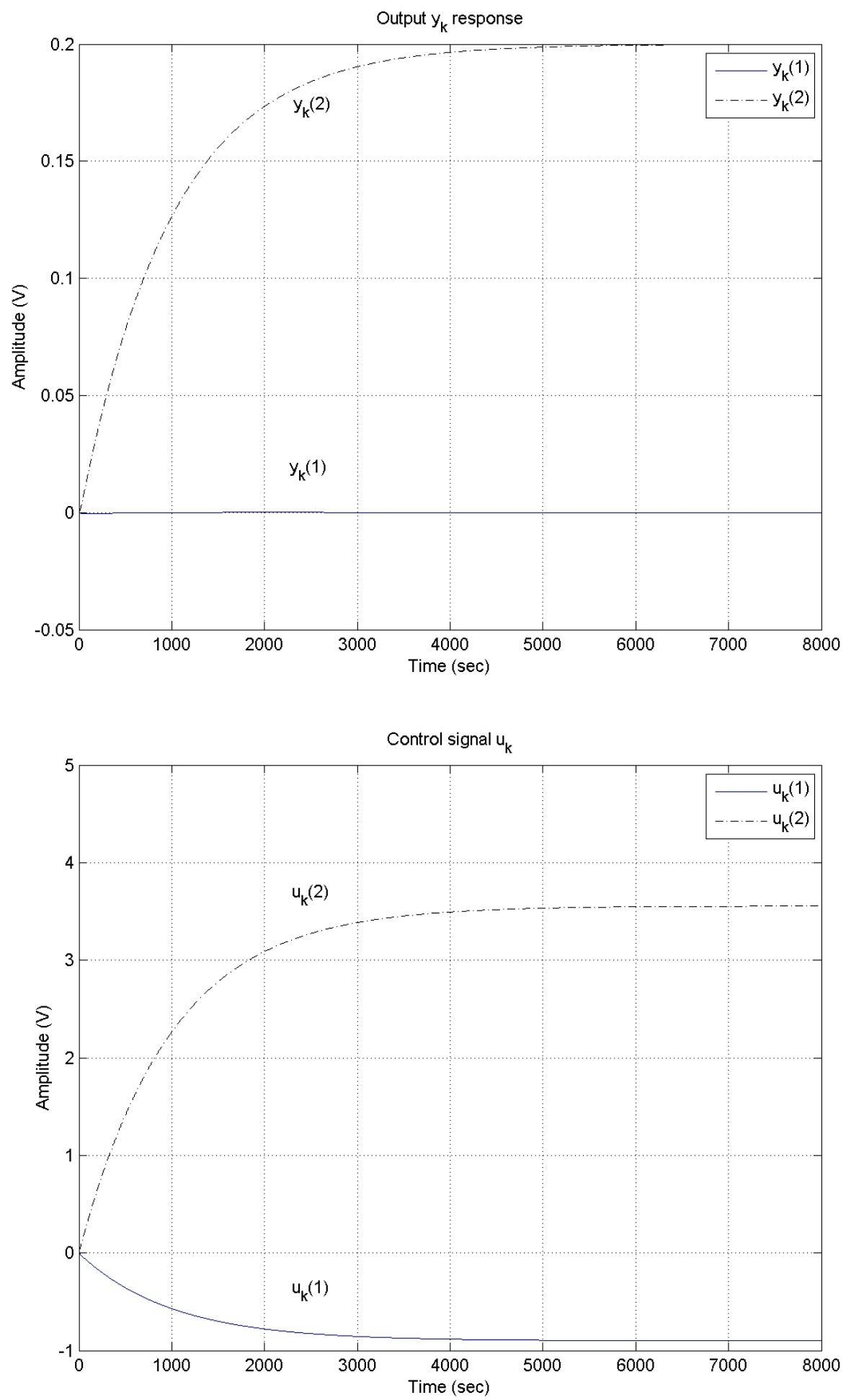

Figure 4.3-2: MARTS Example, Closed-Loop Response

Tuning Regulator (2.17) with $\epsilon=10^{-3}$

Input $y_{\text {ref }}=\left[\begin{array}{ll}0 & 0.2\end{array}\right]^{\top}$

Design 1. 

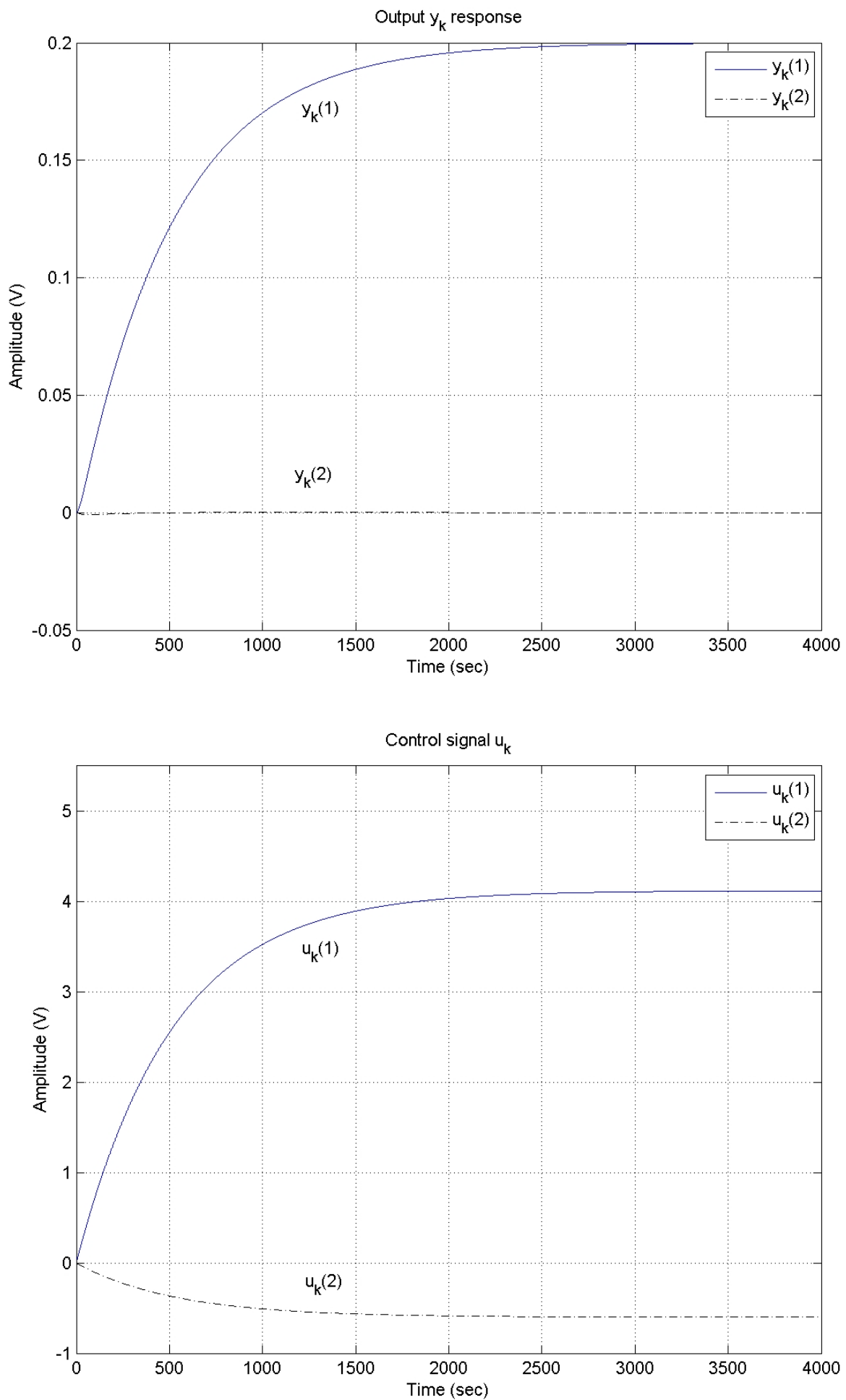

Figure 4.3-3: MARTS Example, Closed-Loop Response Two-Term Controller (2.6) with Controller Gains (4.2)

Input $y_{\text {ref }}=\left[\begin{array}{ll}0.2 & 0\end{array}\right]^{\top}$ Design 1. 

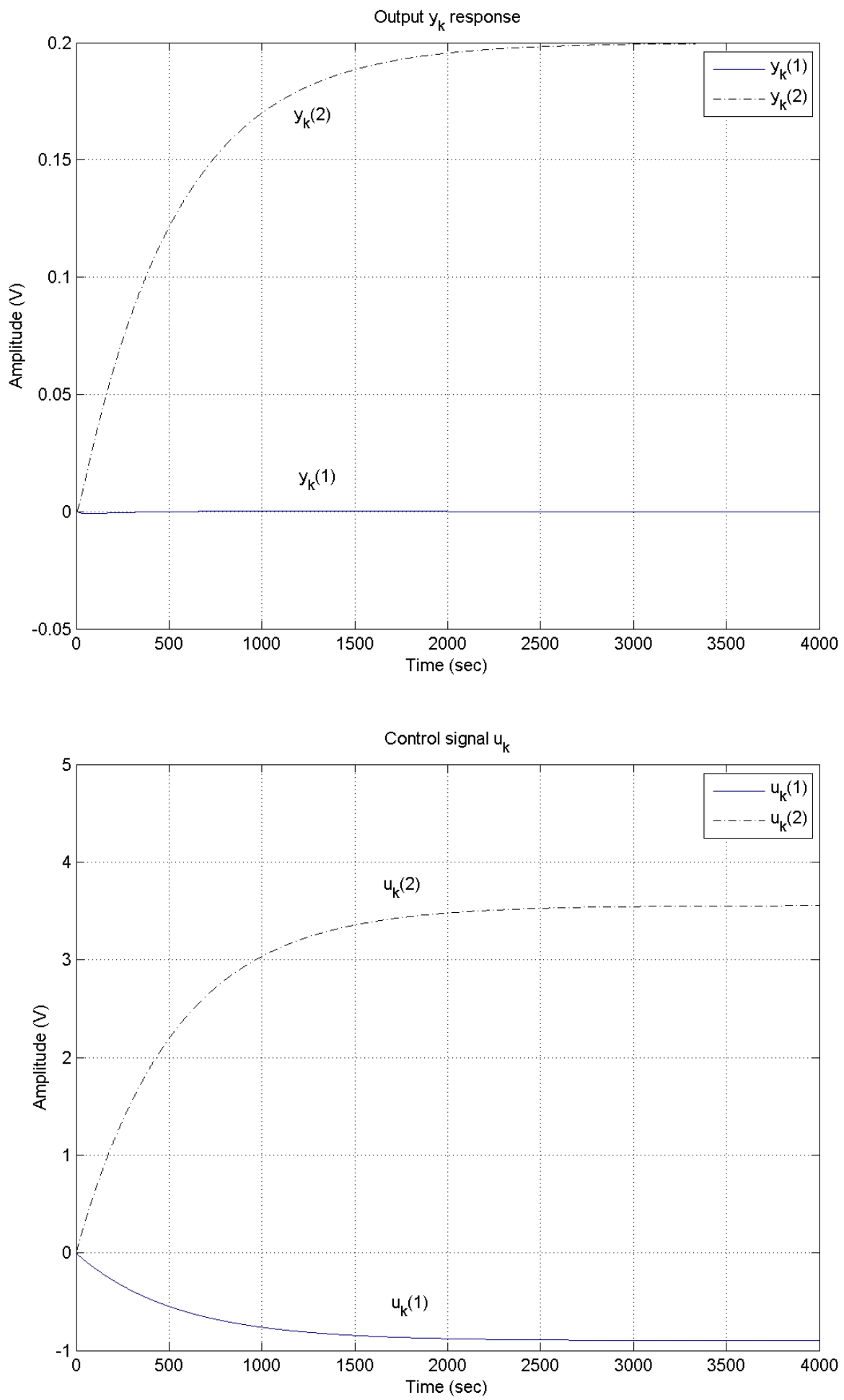

Figure 4.3-4: MARTS Example, Closed-Loop Response Two-Term Controller (2.6) with Controller Gains (4.2) Input $y_{\text {ref }}=\left[\begin{array}{ll}0 & 0.2\end{array}\right]^{\top}$ Design 1. 


\section{Design 1:}

\begin{tabular}{|c|c|c|c|c|}
\hline \multicolumn{5}{|c|}{ Algorithm run \# 1} \\
\hline Starting KP & Starting KI & theta & $\mathrm{mu}$ & Duration $\mathrm{T}$ (sec) \\
\hline 0.0005 & $1 e-005$ & 0 & 1000 & 14000 \\
\hline Iteration & Func-count & $\min f(x)$ & & Procedure \\
\hline 0 & 4 & 20.1123 & & \\
\hline 1 & 12 & 20.0702 & & initial simplex \\
\hline 2 & 20 & 20.0204 & & expand \\
\hline 3 & 28 & 19.9511 & & expand \\
\hline 4 & 36 & 19.8197 & & expand \\
\hline 5 & 44 & 19.6224 & & expand \\
\hline 6 & 52 & 19.2823 & & expand \\
\hline 7 & 60 & 18.7713 & & expand \\
\hline 8 & 68 & 17.9822 & & expand \\
\hline 9 & 76 & 16.9163 & & expand \\
\hline 10 & 84 & 15.6248 & & expand \\
\hline 11 & 92 & 14.497 & & expand \\
\hline 12 & 100 & 14.2989 & & expand \\
\hline 13 & 108 & 14.2989 & & contract \\
\hline 14 & 116 & 14.198 & & contract \\
\hline 15 & 120 & 14.198 & & reflect \\
\hline 16 & 136 & 14.198 & & shrink \\
\hline 17 & 144 & 14.1929 & & contract \\
\hline 18 & 148 & 14.1929 & & reflect \\
\hline 19 & 156 & 14.1927 & & contract \\
\hline 20 & 164 & 14.1926 & & contract \\
\hline 21 & 172 & 14.1923 & & contract \\
\hline 22 & 176 & 14.1923 & & reflect \\
\hline 23 & 192 & 14.1923 & & shrink \\
\hline 24 & 200 & 14.1923 & & contract \\
\hline 25 & 208 & 14.1923 & & contract \\
\hline 26 & 216 & 14.1923 & & contract \\
\hline 27 & 220 & 14.1923 & & reflect \\
\hline 28 & 228 & 14.1923 & & contract \\
\hline 29 & 244 & 14.1923 & & shrink \\
\hline 30 & 252 & 14.1923 & & contract \\
\hline 31 & 260 & 14.1923 & & contract \\
\hline 32 & 268 & 14.1923 & & expand \\
\hline 33 & 272 & 14.1923 & & reflect \\
\hline 34 & 280 & 14.1923 & & expand \\
\hline 35 & 288 & 14.1923 & & contract \\
\hline 36 & 296 & 14.1923 & & expand \\
\hline 37 & 304 & 14.1923 & & expand \\
\hline 38 & 312 & 14.1923 & & expand \\
\hline 39 & 316 & 14.1923 & & reflect \\
\hline 40 & 324 & 14.1923 & & expand \\
\hline 41 & 332 & 14.1923 & & expand \\
\hline 42 & 340 & 14.1923 & & expand \\
\hline 43 & 348 & 14.1923 & & expand \\
\hline 44 & 352 & 14.1923 & & reflect \\
\hline
\end{tabular}




\begin{tabular}{|c|c|c|c|}
\hline 45 & 360 & 14.1923 & expand \\
\hline 46 & 368 & 14.1923 & expand \\
\hline 47 & 376 & 14.1923 & reflect \\
\hline 48 & 384 & 14.1923 & contract \\
\hline 49 & 388 & 14.1923 & reflect \\
\hline 50 & 396 & 14.1923 & contract \\
\hline 51 & 404 & 14.1923 & contract \\
\hline 52 & 412 & 14.1923 & contract \\
\hline 53 & 420 & 14.1923 & contract \\
\hline 54 & 428 & 14.1923 & contract \\
\hline 55 & 432 & 14.1923 & reflect \\
\hline 56 & 440 & 14.1923 & contract \\
\hline 57 & 448 & 14.1923 & contract \\
\hline 58 & 456 & 14.1923 & contract \\
\hline 59 & 464 & 14.1923 & contract \\
\hline 60 & 472 & 14.1923 & contract \\
\hline 61 & 480 & 14.1923 & contract \\
\hline 62 & 488 & 14.1923 & contract \\
\hline 63 & 496 & 14.1923 & reflect \\
\hline 64 & 504 & 14.1923 & reflect \\
\hline 65 & 512 & 14.1923 & contract \\
\hline 66 & 520 & 14.1923 & contract \\
\hline 67 & 536 & 14.1923 & shrink \\
\hline 68 & 544 & 14.1923 & contract \\
\hline 69 & 552 & 14.1923 & contract \\
\hline 70 & 560 & 14.1923 & contract \\
\hline 71 & 568 & 14.1923 & contract \\
\hline 72 & 576 & 14.1923 & contract \\
\hline 73 & 584 & 14.1923 & contract \\
\hline Optimal KP & Optimal KI & Optimal cost $\mathrm{J}$ & \\
\hline 0.0344746 & 0.000936862 & 14.1923 & \\
\hline Algorithm run & 1 \# 2 & & \\
\hline Starting $\mathrm{KP}$ & Starting KI & theta & Duration $\mathrm{T}$ (sec) \\
\hline 0.0344746 & 0.000936862 & 1000 & 14000 \\
\hline Iteration & Func-count & $\min f(x)$ & Procedure \\
\hline 0 & 4 & 14.1923 & \\
\hline 1 & 12 & 14.1923 & initial simplex \\
\hline 2 & 20 & 14.1923 & contract \\
\hline 3 & 36 & 14.1923 & shrink \\
\hline 4 & 52 & 14.1923 & shrink \\
\hline 5 & 68 & 14.1923 & shrink \\
\hline 6 & 84 & 14.1923 & shrink \\
\hline 7 & 100 & 14.1923 & shrink \\
\hline 8 & 116 & 14.1923 & shrink \\
\hline 9 & 132 & 14.1923 & shrink \\
\hline 10 & 148 & 14.1923 & shrink \\
\hline 11 & 164 & 14.1923 & shrink \\
\hline 12 & 180 & 14.1923 & shrink \\
\hline 13 & 196 & 14.1923 & shrink \\
\hline 14 & 212 & 14.1923 & shrink \\
\hline
\end{tabular}


15

Optimal KP

0.0344746
228

Optimal KI 0.000936862
14.1923

Optimal cost $\mathrm{J}$

14.1923

\subsection{Control Design 2 for MARTS}

The tuning regulator (2.17) with $\epsilon=10^{-3}$ is first applied to the plant and the two-term controller (2.6) is then obtained by minimizing the performance index $J_{B \eta_{0}}(2.79)$ using $\theta=0, \mu=10^{3}$. The optimization is carried out under the system settings of:

$$
x_{0}=\left[\begin{array}{l}
0 \\
0
\end{array}\right], y_{r e f}=\left[\begin{array}{l}
0 \\
0
\end{array}\right], \text { and } \mathcal{Z}_{\eta_{0}}=\left\{\left[\begin{array}{c}
0 \\
0.2
\end{array}\right],\left[\begin{array}{c}
\frac{0.2}{\sqrt{2}} \\
\frac{0.2}{\sqrt{2}}
\end{array}\right],\left[\begin{array}{c}
0.2 \\
0
\end{array}\right],\left[\begin{array}{c}
\frac{0.2}{\sqrt{2}} \\
-\frac{0.2}{\sqrt{2}}
\end{array}\right]\right\} \text {. }
$$

The above settings correspond to approximating the performance index $J_{B \eta_{0}}(2.79)$; in this case the optimal control gains $K_{P}$ and $K_{I}$ are found to be

$$
K_{P}=3.4475 \times 10^{-2}, \quad K_{I}=9.3686 \times 10^{-4} .
$$

Simulation of plant's initial condition input is given in Figures 4.4-1 to 4.4-4 for the resulting closed-loop system.

Remark 4.4. The controller found by minimizing $J_{B \eta_{0}}(2.79)$ is identical to that obtained by minimizing $J_{A y_{\text {ref }}}(2.59)$. The approach used in Control Design 2 has the advantage of being able to tune controllers without perturbing the plant's initial states, changing tracking set-points, or injecting disturbances into the system. 

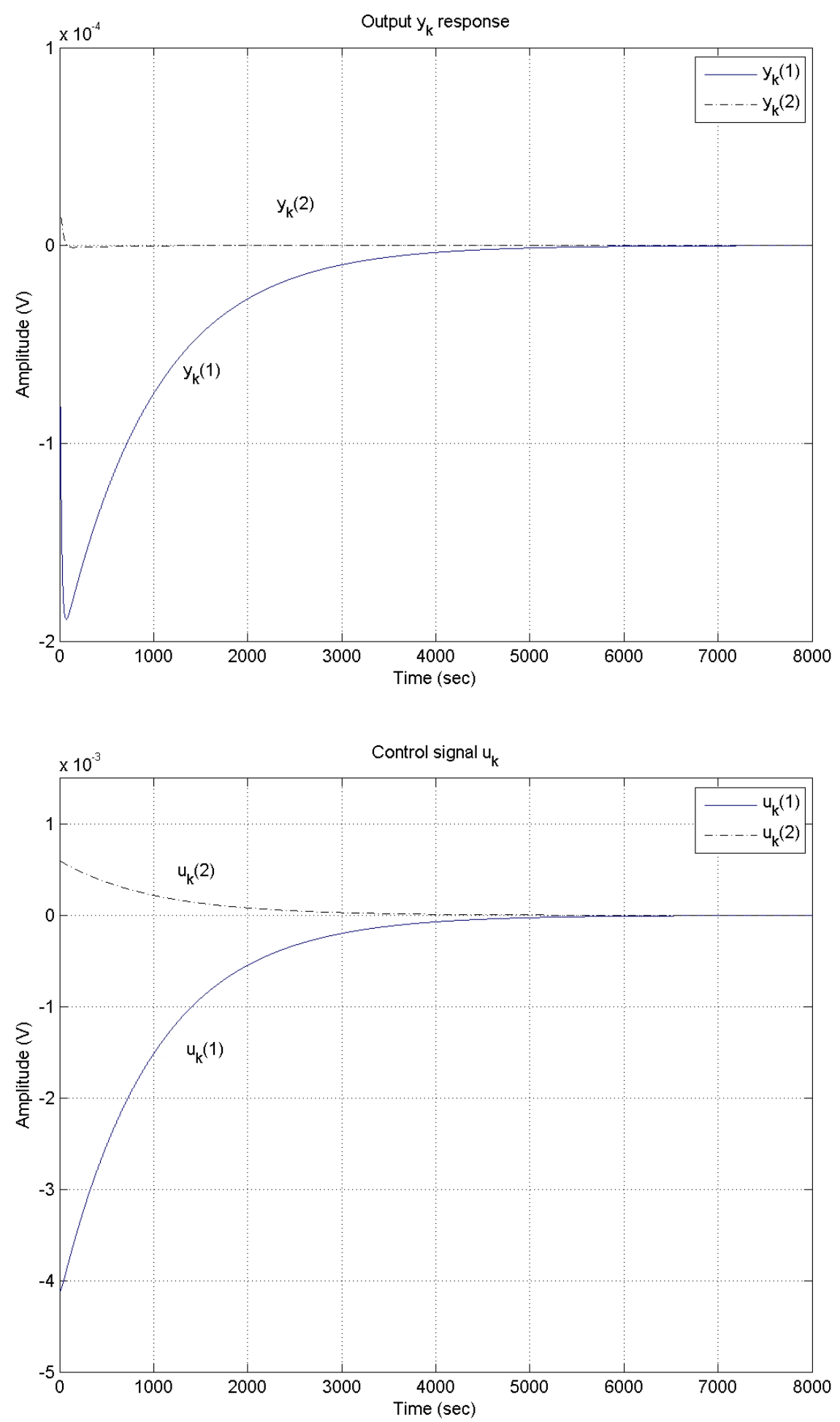

Figure 4.4-1: MARTS Example, Closed-Loop Response Tuning Regulator (2.17) with $\epsilon=10^{-3}$ Input $\eta_{0}=\left[\begin{array}{ll}0.2 & 0\end{array}\right]^{\top}$

Design 2 . 

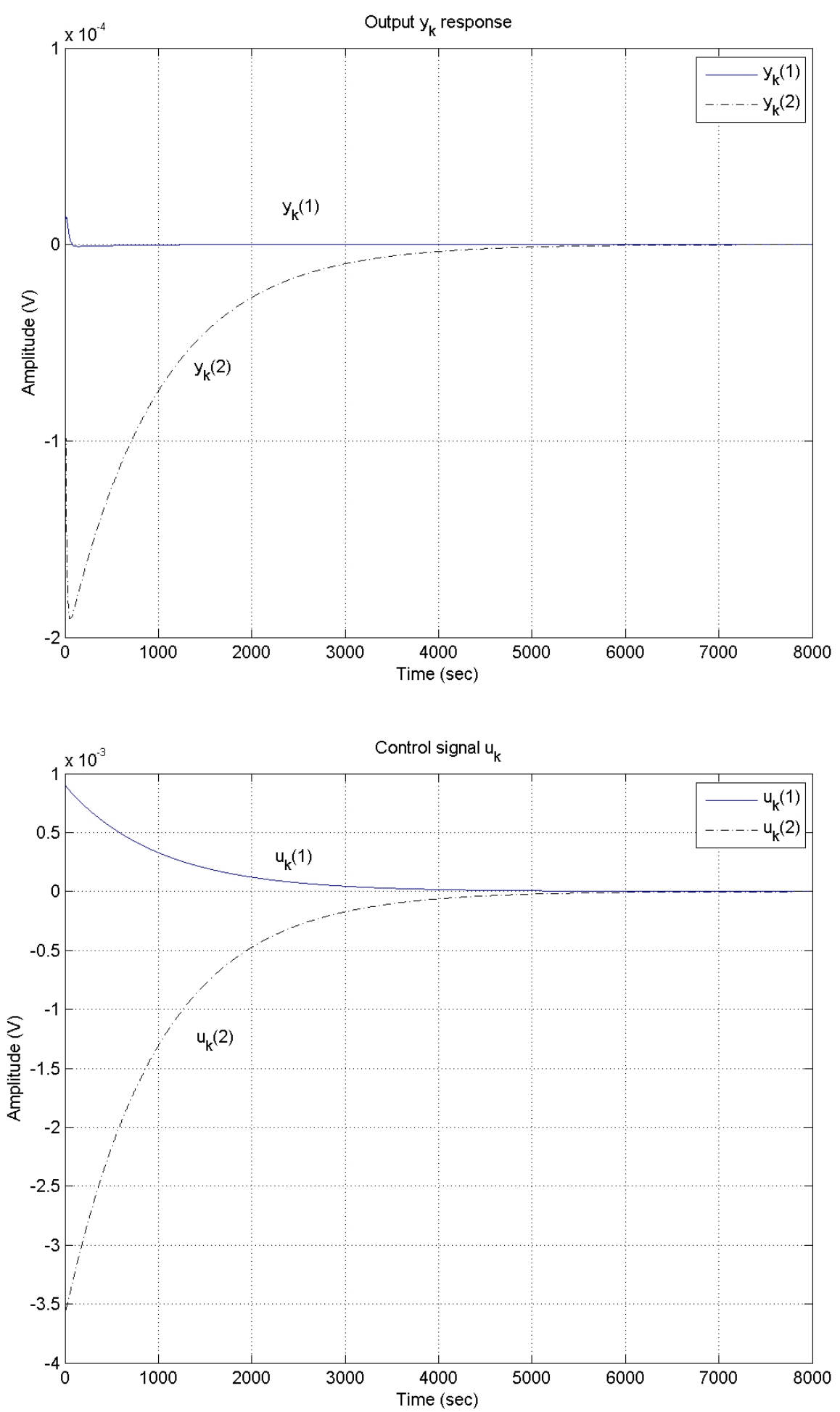

Figure 4.4-2: MARTS Example, Closed-Loop Response Tuning Regulator (2.17) with $\epsilon=10^{-3}$ Input $\eta_{0}=\left[\begin{array}{ll}0 & 0.2\end{array}\right]^{\top}$

Design 2 . 

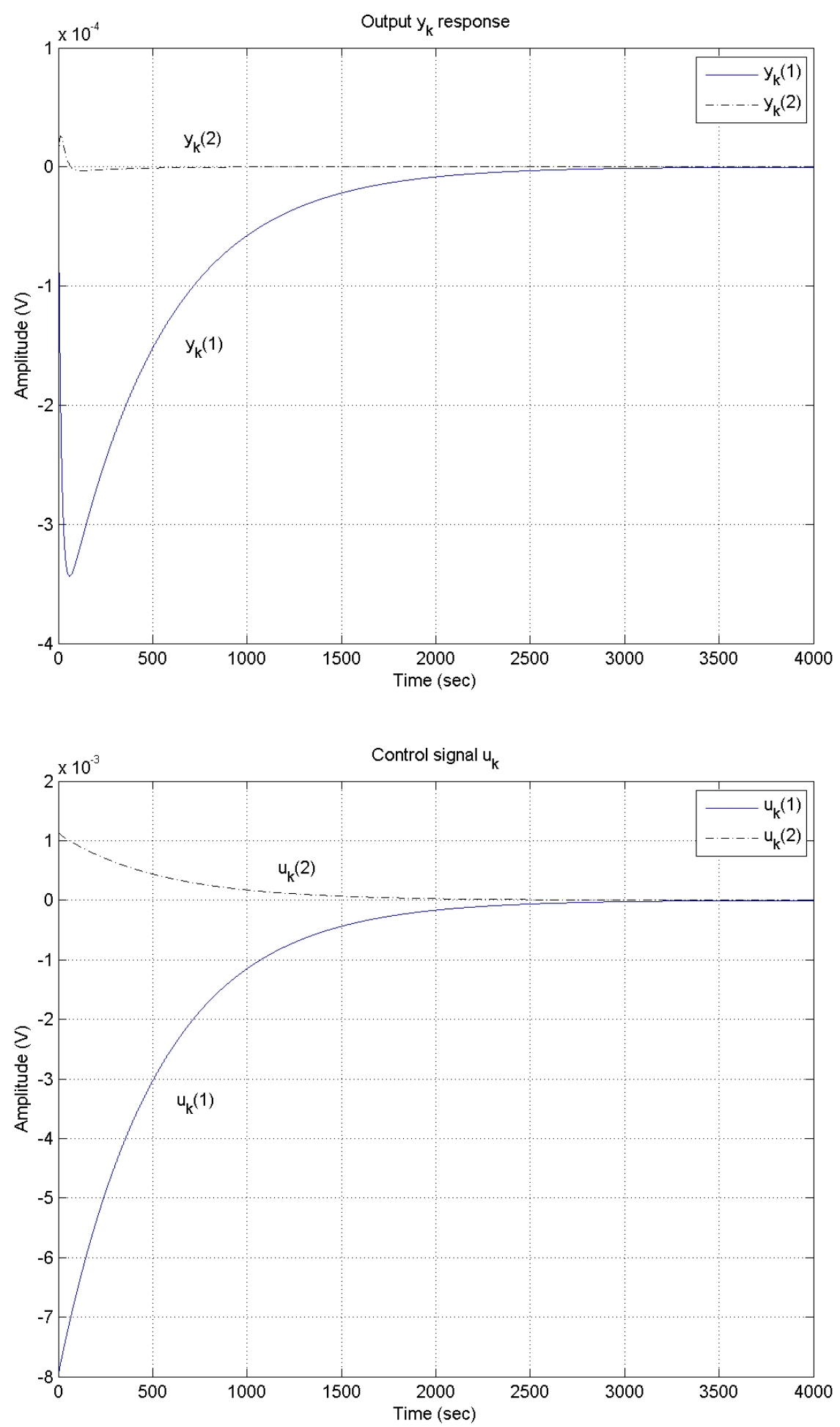

Figure 4.4-3: MARTS Example, Closed-Loop Response Two-Term Controller (2.6) with Controller Gains (4.3) Input $\eta_{0}=\left[\begin{array}{ll}0.2 & 0\end{array}\right]^{\top}$

Design 2 . 

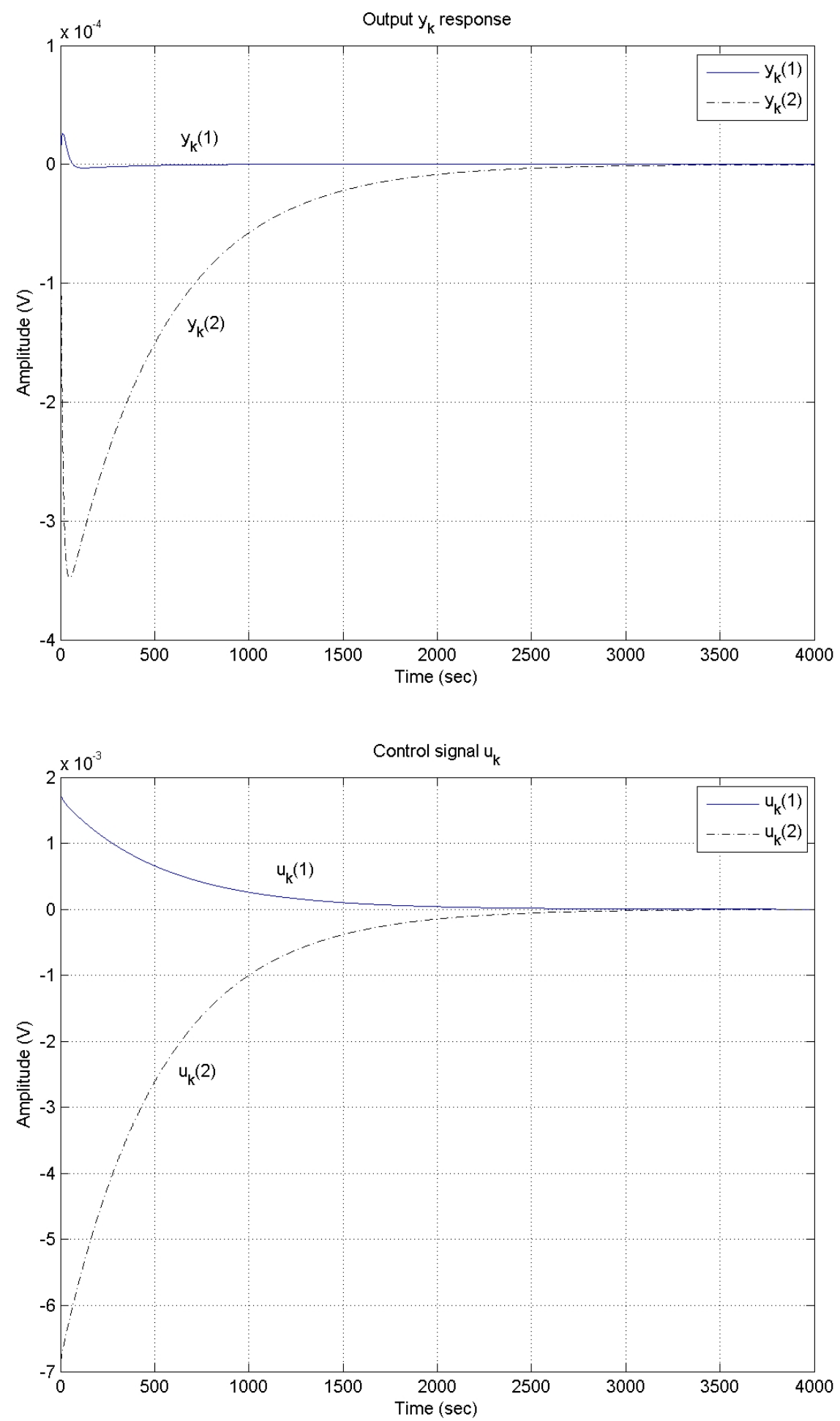

Figure 4.4-4: MARTS Example, Closed-Loop Response Two-Term Controller (2.6) with Controller Gains (4.3) Input $\eta_{0}=\left[\begin{array}{ll}0 & 0.2\end{array}\right]^{\top}$

Design 2 . 


\section{Design 2:}

\begin{tabular}{|c|c|c|c|c|}
\hline \multicolumn{5}{|c|}{ Algorithm run \# 1} \\
\hline Starting KP & Starting KI & theta & $\mathrm{mu}$ & Duration $\mathrm{T}$ (sec) \\
\hline 0.0005 & $1 e-005$ & 0 & 1000 & 14000 \\
\hline Iteration & Func-count & $\min f(x)$ & & Procedure \\
\hline 0 & 4 & 20.1122 & & \\
\hline 1 & 12 & 20.0701 & & initial simplex \\
\hline 2 & 20 & 20.0203 & & expand \\
\hline 3 & 28 & 19.951 & & expand \\
\hline 4 & 36 & 19.8197 & & expand \\
\hline 5 & 44 & 19.6224 & & expand \\
\hline 6 & 52 & 19.2823 & & expand \\
\hline 7 & 60 & 18.7713 & & expand \\
\hline 8 & 68 & 17.9822 & & expand \\
\hline 9 & 76 & 16.9163 & & expand \\
\hline 10 & 84 & 15.6248 & & expand \\
\hline 11 & 92 & 14.497 & & expand \\
\hline 12 & 100 & 14.2989 & & expand \\
\hline 13 & 108 & 14.2989 & & contract \\
\hline 14 & 116 & 14.198 & & contract \\
\hline 15 & 120 & 14.198 & & reflect \\
\hline 16 & 136 & 14.198 & & shrink \\
\hline 17 & 144 & 14.1929 & & contract \\
\hline 18 & 148 & 14.1929 & & reflect \\
\hline 19 & 156 & 14.1927 & & contract \\
\hline 20 & 164 & 14.1926 & & contract \\
\hline 21 & 172 & 14.1923 & & contract \\
\hline 22 & 176 & 14.1923 & & reflect \\
\hline 23 & 192 & 14.1923 & & shrink \\
\hline 24 & 200 & 14.1923 & & contract \\
\hline 25 & 208 & 14.1923 & & contract \\
\hline 26 & 216 & 14.1923 & & contract \\
\hline 27 & 220 & 14.1923 & & reflect \\
\hline 28 & 228 & 14.1923 & & contract \\
\hline 29 & 244 & 14.1923 & & shrink \\
\hline 30 & 252 & 14.1923 & & contract \\
\hline 31 & 260 & 14.1923 & & contract \\
\hline 32 & 268 & 14.1923 & & expand \\
\hline 33 & 272 & 14.1923 & & reflect \\
\hline 34 & 280 & 14.1923 & & expand \\
\hline 35 & 288 & 14.1923 & & contract \\
\hline 36 & 296 & 14.1923 & & expand \\
\hline 37 & 304 & 14.1923 & & expand \\
\hline 38 & 312 & 14.1923 & & expand \\
\hline 39 & 316 & 14.1923 & & reflect \\
\hline 40 & 324 & 14.1923 & & expand \\
\hline 41 & 332 & 14.1923 & & expand \\
\hline 42 & 340 & 14.1923 & & expand \\
\hline 43 & 348 & 14.1923 & & expand \\
\hline 44 & 352 & 14.1923 & & reflect \\
\hline
\end{tabular}




\begin{tabular}{|c|c|c|c|}
\hline 45 & 360 & 14.1923 & expand \\
\hline 46 & 368 & 14.1923 & expand \\
\hline 47 & 376 & 14.1923 & reflect \\
\hline 48 & 384 & 14.1923 & contract \\
\hline 49 & 388 & 14.1923 & reflect \\
\hline 50 & 396 & 14.1923 & contract \\
\hline 51 & 404 & 14.1923 & contract \\
\hline 52 & 412 & 14.1923 & contract \\
\hline 53 & 420 & 14.1923 & contract \\
\hline 54 & 428 & 14.1923 & contract \\
\hline 55 & 432 & 14.1923 & reflect \\
\hline 56 & 440 & 14.1923 & contract \\
\hline 57 & 448 & 14.1923 & contract \\
\hline 58 & 456 & 14.1923 & contract \\
\hline 59 & 464 & 14.1923 & contract \\
\hline 60 & 472 & 14.1923 & contract \\
\hline 61 & 480 & 14.1923 & contract \\
\hline 62 & 488 & 14.1923 & contract \\
\hline 63 & 492 & 14.1923 & reflect \\
\hline 64 & 500 & 14.1923 & reflect \\
\hline 65 & 508 & 14.1923 & contract \\
\hline 66 & 516 & 14.1923 & contract \\
\hline 67 & 524 & 14.1923 & contract \\
\hline 68 & 532 & 14.1923 & contract \\
\hline 69 & 540 & 14.1923 & contract \\
\hline 70 & 548 & 14.1923 & contract \\
\hline 71 & 556 & 14.1923 & reflect \\
\hline 72 & 564 & 14.1923 & contract \\
\hline 73 & 572 & 14.1923 & contract \\
\hline 74 & 580 & 14.1923 & contract \\
\hline 75 & 588 & 14.1923 & contract \\
\hline Optimal KP & Optimal KI & Optimal cost $\mathrm{J}$ & \\
\hline 0.034475 & 0.000936863 & 14.1923 & \\
\hline Algorithm run & $n \# 2$ & & \\
\hline Starting KP & Starting KI & theta & Duration $\mathrm{T}$ (sec) \\
\hline 0.034475 & 0.000936863 & 1000 & 14000 \\
\hline Iteration & Func-count & $\min f(x)$ & Procedure \\
\hline 0 & 4 & 14.1923 & \\
\hline 1 & 12 & 14.1923 & initial simplex \\
\hline 2 & 20 & 14.1923 & contract \\
\hline 3 & 36 & 14.1923 & shrink \\
\hline 4 & 52 & 14.1923 & shrink \\
\hline 5 & 68 & 14.1923 & shrink \\
\hline 6 & 84 & 14.1923 & shrink \\
\hline 7 & 100 & 14.1923 & shrink \\
\hline 8 & 116 & 14.1923 & shrink \\
\hline 9 & 132 & 14.1923 & shrink \\
\hline 10 & 148 & 14.1923 & shrink \\
\hline 11 & 164 & 14.1923 & shrink \\
\hline 12 & 180 & 14.1923 & shrink \\
\hline
\end{tabular}




$\begin{array}{cccc}13 & 196 & 14.1923 & \text { shrink } \\ 14 & 212 & 14.1923 & \text { shrink } \\ 15 & 228 & 14.1923 & \text { shrink } \\ & & & \\ \text { Optimal KP } & \text { Optimal KI } & \text { Optimal cost J } & \\ 0.034475 & 0.000936863 & 14.1923 & \\ & & & \end{array}$

\subsection{Control Design 1 for MARTS Repeated under the Influence of Noise}

The effectiveness of the design approach under the influence of noise in the outputs of the system can be briefly investigated by introducing an additive white Gaussian noise (AWGN) channel with 0 mean and a variance of $5 \times 10^{-6}$ at the output of the plant. The two-term controller (2.6) is obtained by minimizing the performance index $J_{A y_{r e f}}$ (2.59) using $\theta=0, \mu=10^{3}$. The optimization is carried out under the system settings of:

$$
x_{0}=\left[\begin{array}{l}
0 \\
0
\end{array}\right], \eta_{0}=\left[\begin{array}{l}
0 \\
0
\end{array}\right] \text {, and } \mathcal{Z}_{y_{r e f}}=\left\{\left[\begin{array}{c}
0 \\
0.2
\end{array}\right],\left[\begin{array}{c}
\frac{0.2}{\sqrt{2}} \\
\frac{0.2}{\sqrt{2}}
\end{array}\right],\left[\begin{array}{c}
0.2 \\
0
\end{array}\right],\left[\begin{array}{c}
\frac{0.2}{\sqrt{2}} \\
-\frac{0.2}{\sqrt{2}}
\end{array}\right]\right\}
$$

in this case the optimal control gains $K_{P}$ and $K_{I}$ are found to be

$$
K_{P}=4.6517 \times 10^{-5}, \quad K_{I}=8.5770 \times 10^{-4} .
$$

Simulation of tracking reference input is given in Figures 4.5-1 and 4.5-2 for the resulting closed-loop system.

Remark 4.5. The mean and variance values of the white noise signal are obtained from experimental measurements made on MARTS.

Remark 4.6. The initial Nelder-Mead simplex is enlarged so that the performance of the various controllers being compared can be distinguished from each other.

Remark 4.7. The optimal control gains are significantly smaller than those obtained when noise was absent. As previously discussed, the structure of performance index $J_{A}$ (2.102) is sensitive to noise with the result that Nelder-Mead converges prematurely to a non-stationary point. 

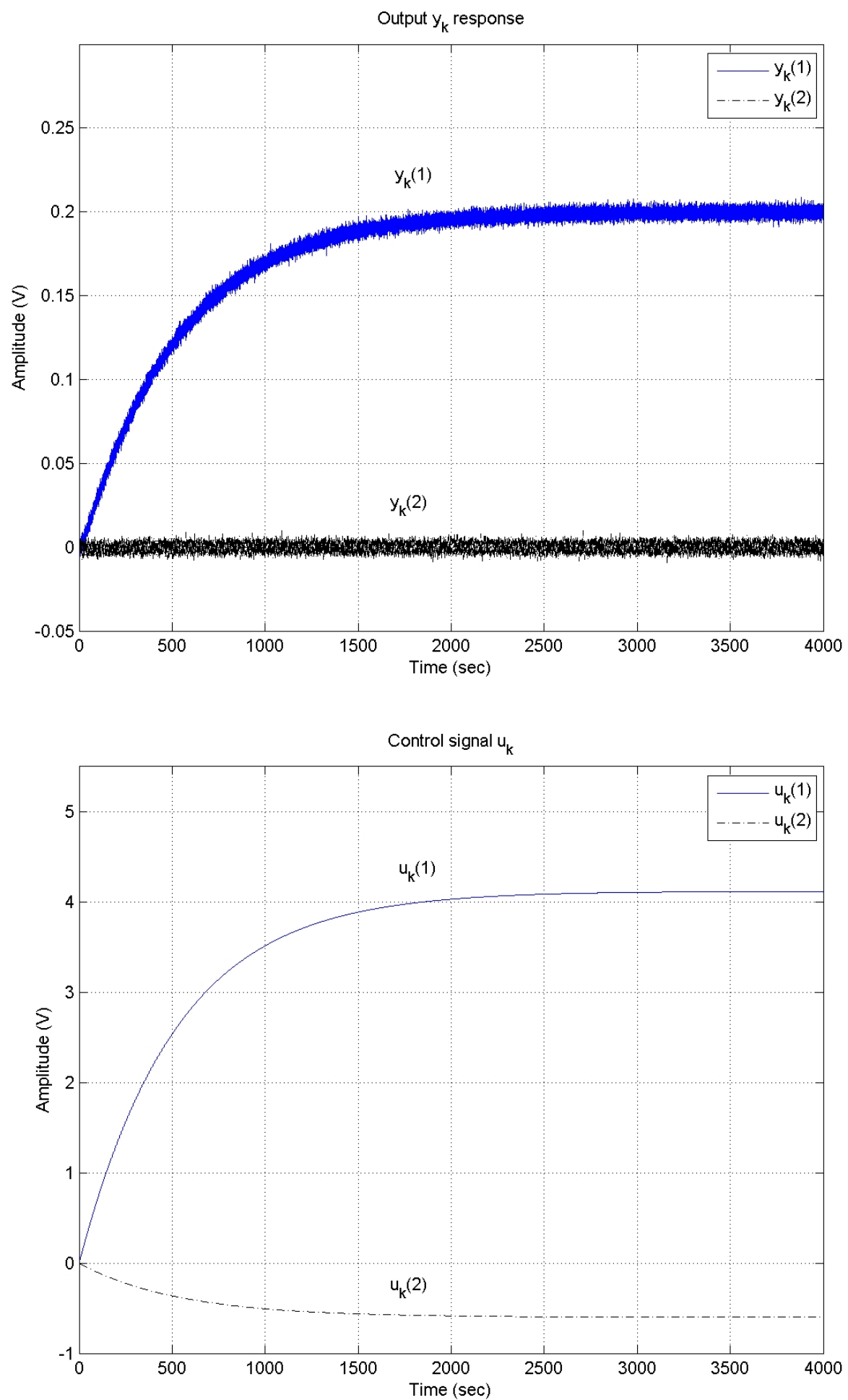

Figure 4.5-1: MARTS Example with Noise, Closed-Loop Response

Two-Term Controller (2.6) with Controller Gains (4.4)

Input $y_{\text {ref }}=\left[\begin{array}{ll}0.2 & 0\end{array}\right]^{\top}$

Design 1 with Noise. 

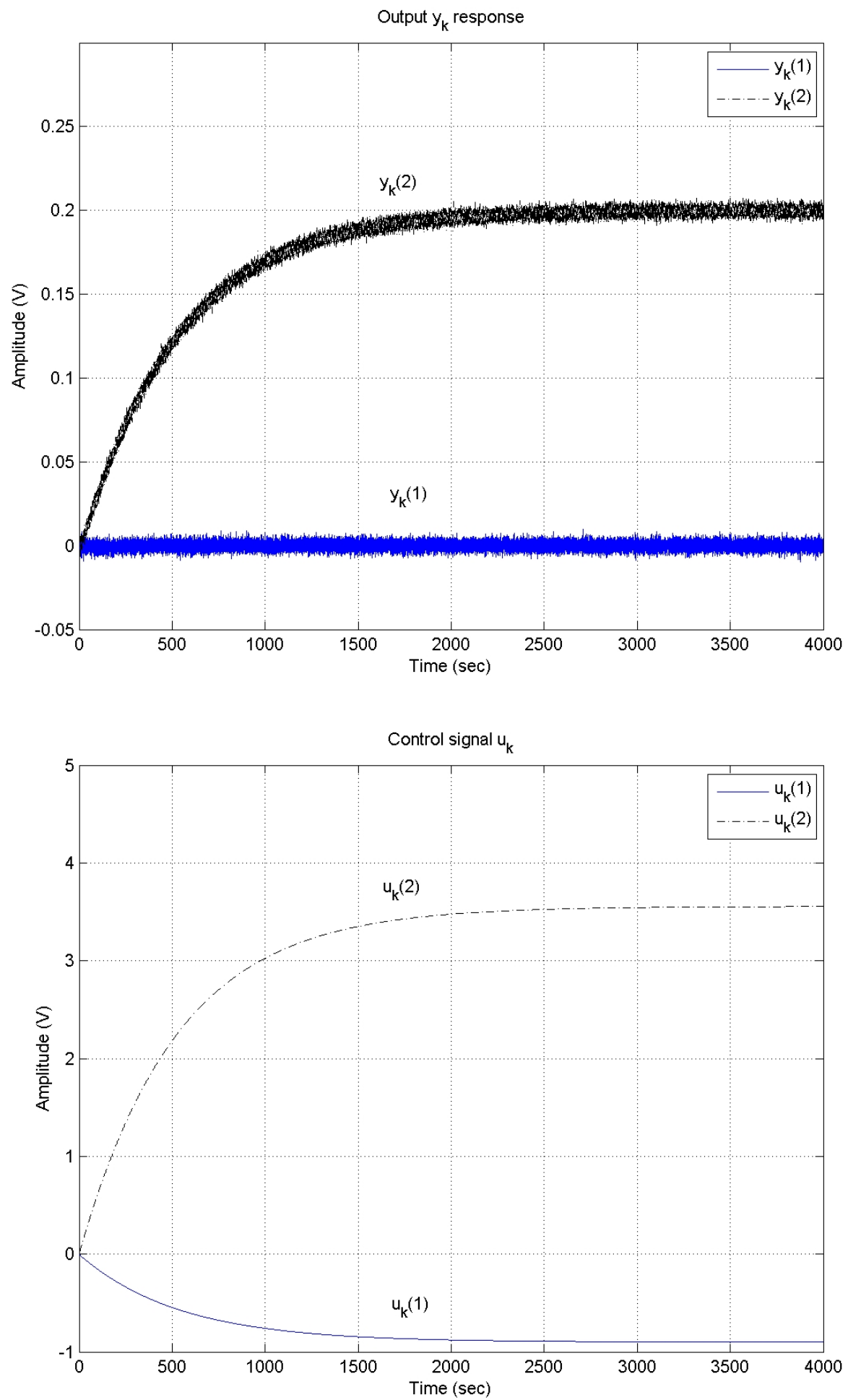

Figure 4.5-2: MARTS Example with Noise, Closed-Loop Response

Two-Term Controller (2.6) with Controller Gains (4.4)

Input $y_{\text {ref }}=\left[\begin{array}{ll}0 & 0.2\end{array}\right]^{\top}$

Design 1 with Noise. 
Design 1 with Noise:

\begin{tabular}{|c|c|c|c|c|}
\hline \multicolumn{5}{|c|}{ Algorithm run \# 1} \\
\hline $\begin{array}{c}\text { Starting KP } \\
0.0005\end{array}$ & $\begin{array}{l}\text { Starting KI } \\
\text { 1e-005 }\end{array}$ & $\begin{array}{c}\text { theta } \\
0\end{array}$ & $\begin{array}{c}\mathrm{mu} \\
1000\end{array}$ & $\begin{array}{l}\text { Duration } \mathrm{T} \text { (sec) } \\
\quad 14000\end{array}$ \\
\hline Iteration & Func-count & $\min f(x)$ & & Procedure \\
\hline 0 & 4 & 22.9474 & & \\
\hline 1 & 12 & 22.9474 & & initial simplex \\
\hline 2 & 20 & 22.5605 & & reflect \\
\hline 3 & 28 & 22.5561 & & contract \\
\hline 4 & 36 & 22.2176 & & reflect \\
\hline 5 & 44 & 22.1868 & & reflect \\
\hline 6 & 52 & 21.8889 & & reflect \\
\hline 7 & 60 & 21.8297 & & reflect \\
\hline 8 & 68 & 21.584 & & reflect \\
\hline 9 & 76 & 21.504 & & reflect \\
\hline 10 & 84 & 21.2949 & & reflect \\
\hline 11 & 92 & 21.1885 & & reflect \\
\hline 12 & 100 & 21.0137 & & reflect \\
\hline 13 & 108 & 20.8959 & & reflect \\
\hline 14 & 116 & 20.7519 & & reflect \\
\hline 15 & 124 & 20.599 & & reflect \\
\hline 16 & 132 & 20.5038 & & reflect \\
\hline 17 & 140 & 20.3408 & & reflect \\
\hline 18 & 148 & 20.2697 & & reflect \\
\hline 19 & 156 & 20.0829 & & reflect \\
\hline 20 & 164 & 20.0565 & & reflect \\
\hline 21 & 172 & 19.8427 & & reflect \\
\hline 22 & 176 & 19.8427 & & reflect \\
\hline 23 & 184 & 19.6156 & & reflect \\
\hline 24 & 192 & 17.0916 & & contract \\
\hline 25 & 200 & 16.8835 & & reflect \\
\hline 26 & 216 & 16.8835 & & shrink \\
\hline 27 & 232 & 16.8835 & & shrink \\
\hline 28 & 248 & 16.8835 & & shrink \\
\hline 29 & 256 & 16.8835 & & contract \\
\hline 30 & 264 & 16.8701 & & expand \\
\hline 31 & 272 & 16.8111 & & expand \\
\hline 32 & 280 & 16.7909 & & expand \\
\hline 33 & 288 & 16.6242 & & expand \\
\hline 34 & 292 & 16.6242 & & reflect \\
\hline 35 & 300 & 16.3284 & & expand \\
\hline 36 & 304 & 16.3284 & & reflect \\
\hline 37 & 312 & 15.8196 & & expand \\
\hline 38 & 316 & 15.8196 & & reflect \\
\hline 39 & 324 & 15.0599 & & expand \\
\hline 40 & 328 & 15.0599 & & reflect \\
\hline 41 & 336 & 14.5441 & & expand \\
\hline 42 & 344 & 14.3687 & & expand \\
\hline 43 & 352 & 14.3687 & & contract \\
\hline 44 & 360 & 14.3687 & & contract \\
\hline
\end{tabular}




\begin{tabular}{|c|c|c|c|}
\hline 45 & 364 & 14.3687 & reflect \\
\hline 46 & 368 & 14.3687 & reflect \\
\hline 47 & 376 & 14.3675 & contract \\
\hline 48 & 384 & 14.3632 & contract \\
\hline 49 & 392 & 14.3537 & contract \\
\hline 50 & 396 & 14.3537 & reflect \\
\hline 51 & 412 & 14.3537 & shrink \\
\hline 52 & 420 & 14.3527 & contract \\
\hline 53 & 428 & 14.3527 & contract \\
\hline 54 & 436 & 14.3513 & contract \\
\hline 55 & 452 & 14.3513 & shrink \\
\hline 56 & 456 & 14.3513 & reflect \\
\hline 57 & 464 & 14.3513 & contract \\
\hline 58 & 472 & 14.3506 & reflect \\
\hline 59 & 488 & 14.3506 & shrink \\
\hline 60 & 504 & 14.3506 & shrink \\
\hline 61 & 508 & 14.3506 & reflect \\
\hline 62 & 524 & 14.3506 & shrink \\
\hline Optimal KP & Optimal KI & Optimal cost $\mathrm{J}$ & \\
\hline $4.13171 e-005$ & 0.000871634 & 14.3506 & \\
\hline Algorithm run & $n \# 2$ & & \\
\hline Starting KP & Starting KI & theta & Duration $\mathrm{T}$ (sec) \\
\hline $4.13171 e-005$ & 0.000871634 & 1000 & 5000 \\
\hline Iteration & Func-count & $\min f(x)$ & Procedure \\
\hline 0 & 4 & 14.2495 & \\
\hline 1 & 12 & 14.2495 & initial simplex \\
\hline 2 & 28 & 14.2495 & shrink \\
\hline 3 & 44 & 14.2495 & shrink \\
\hline 4 & 52 & 14.2495 & contract \\
\hline 5 & 60 & 14.2495 & contract \\
\hline 6 & 76 & 14.2495 & shrink \\
\hline 7 & 84 & 14.2495 & contract \\
\hline 8 & 92 & 14.2495 & contract \\
\hline 9 & 100 & 14.2481 & contract \\
\hline 10 & 116 & 14.2481 & shrink \\
\hline 11 & 132 & 14.2481 & shrink \\
\hline 12 & 148 & 14.2481 & shrink \\
\hline 13 & 152 & 14.2481 & reflect \\
\hline 14 & 156 & 14.2481 & reflect \\
\hline 15 & 172 & 14.2481 & shrink \\
\hline 16 & 188 & 14.2481 & shrink \\
\hline Optimal KP & Optimal KI & Optimal cost $\mathrm{J}$ & \\
\hline $4.6517 e-005$ & 0.000857696 & 14.2481 & \\
\hline
\end{tabular}




\subsection{Control Design 2 for MARTS Repeated under the Influence of Noise}

The AWGN channel is again introduced at the output of the plant. The two-term controller (2.6) is obtained by minimizing the performance index $J_{B \eta_{0}}(2.79)$ using $\theta=0, \mu=$ $10^{3}$. The optimization is carried out under the system settings of:

$$
x_{0}=\left[\begin{array}{l}
0 \\
0
\end{array}\right], y_{\text {ref }}=\left[\begin{array}{l}
0 \\
0
\end{array}\right], \text { and } \mathcal{Z}_{\eta_{0}}=\left\{\left[\begin{array}{c}
0 \\
100
\end{array}\right],\left[\begin{array}{c}
\frac{100}{\sqrt{2}} \\
\frac{100}{\sqrt{2}}
\end{array}\right],\left[\begin{array}{c}
100 \\
0
\end{array}\right],\left[\begin{array}{c}
\frac{100}{\sqrt{2}} \\
-\frac{100}{\sqrt{2}}
\end{array}\right]\right\}
$$

in this case the optimal control gains $K_{P}$ and $K_{I}$ are found to be

$$
K_{P}=2.8193 \times 10^{-2}, \quad K_{I}=9.4791 \times 10^{-4}
$$

Simulation of servocompensator's initial condition input is given in Figures 4.6-1 to 4.6-4 for the resulting closed-loop system.

Remark 4.8. The large $\eta_{0}$ values are required to prevent the plant output from being completely masked by noise. It is seen that the tuning regulator with a small $\epsilon$ ensures that the closed-loop system will be unsaturated when $\eta_{0}$ is large. This time, the optimal control gains are approximately the same as those obtained when noise was absent. These results suggest that performance index $J_{B}$ (2.103) should be used when designing controllers for experimental systems if no filters are used.

Remark 4.9. When evaluating a controller's performance for a specific $\eta_{0}$ setting, the experiment is performed only once, as opposed to repeating the same experiment multiple times to find an accurate measure. The motivation is to approach to the vicinity of the optimal point using as few function evaluations as possible.

Remark 4.10. When the Nelder-Mead algorithm converges to a point that is closer to the minimizer, one can restart the algorithm from this point and use a shorter fixed time duration for all subsequent experiments (corresponds to reducing $T$ in the performance index) as the closed-loop system response becomes faster. In this case, a shorter time duration has the benefit of reducing the impact of noise on the cost value at steady state. If the duration of each experiment is much longer than the system's transient response time, then the cost does not well represent the performance of the controller as it is corrupted mostly by the accumulated noise at steady state. 

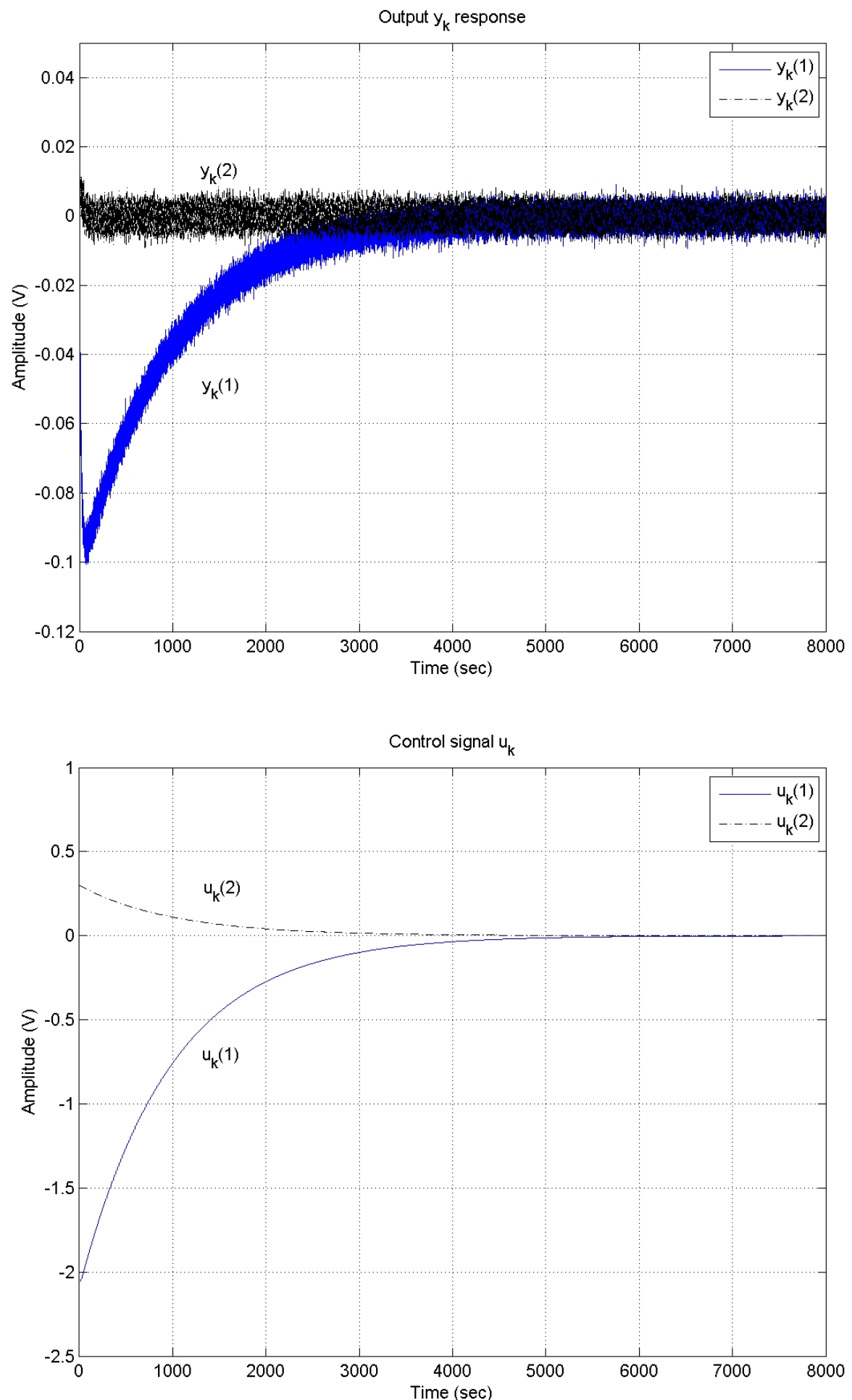

Figure 4.6-1: MARTS Example with Noise, Closed-Loop Response Tuning Regulator (2.17) with $\epsilon=10^{-3}$

Input $\eta_{0}=\left[\begin{array}{ll}100 & 0\end{array}\right]^{\top}$

Design 2 with Noise. 

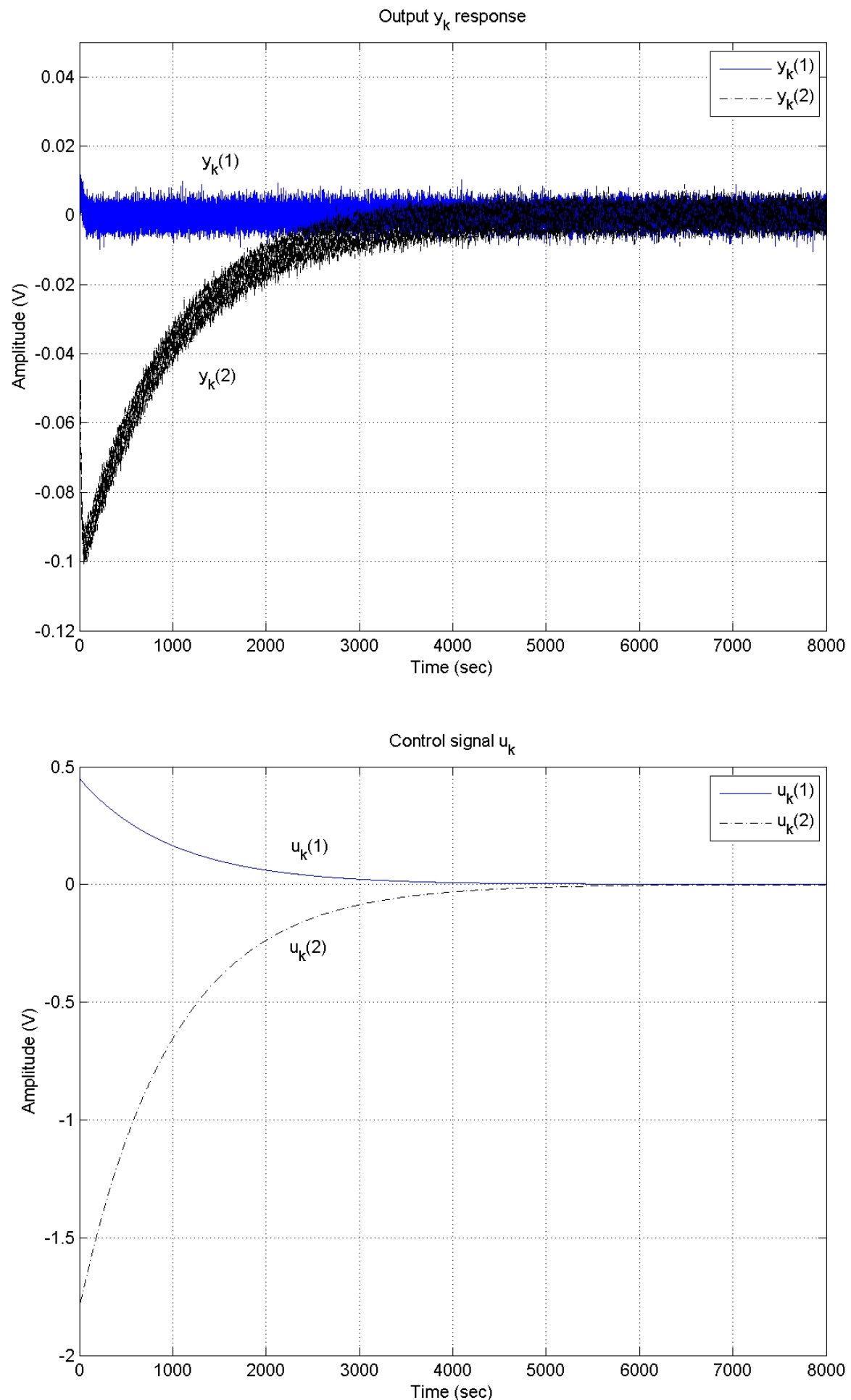

Figure 4.6-2: MARTS Example with Noise, Closed-Loop Response Tuning Regulator (2.17) with $\epsilon=10^{-3}$

Input $\eta_{0}=\left[\begin{array}{ll}0 & 100\end{array}\right]^{\top}$

Design 2 with Noise. 

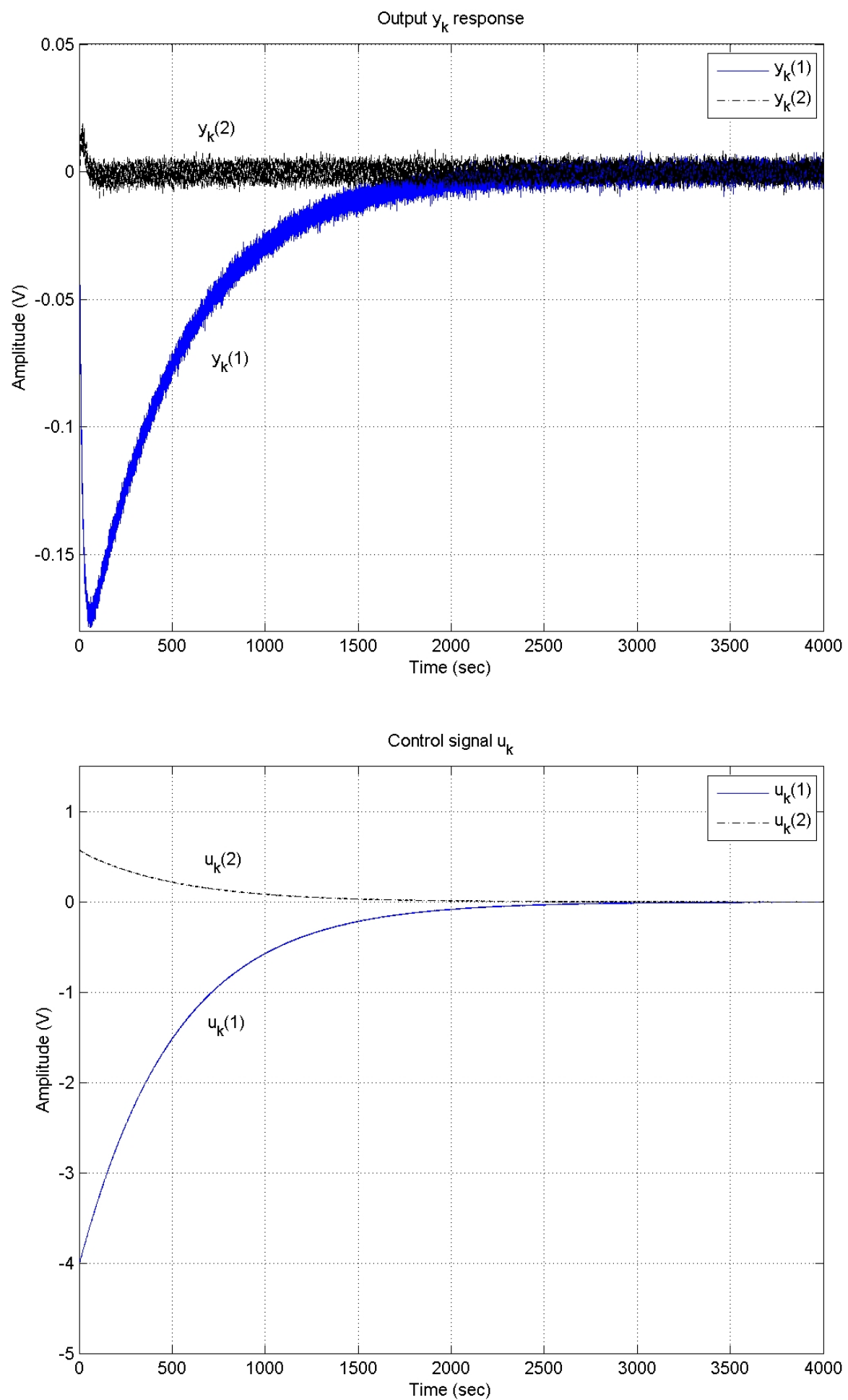

Figure 4.6-3: MARTS Example with Noise, Closed-Loop Response

Two-Term Controller (2.6) with Controller Gains (4.5)

Input $\eta_{0}=\left[\begin{array}{ll}100 & 0\end{array}\right]^{\top}$

Design 2 with Noise. 

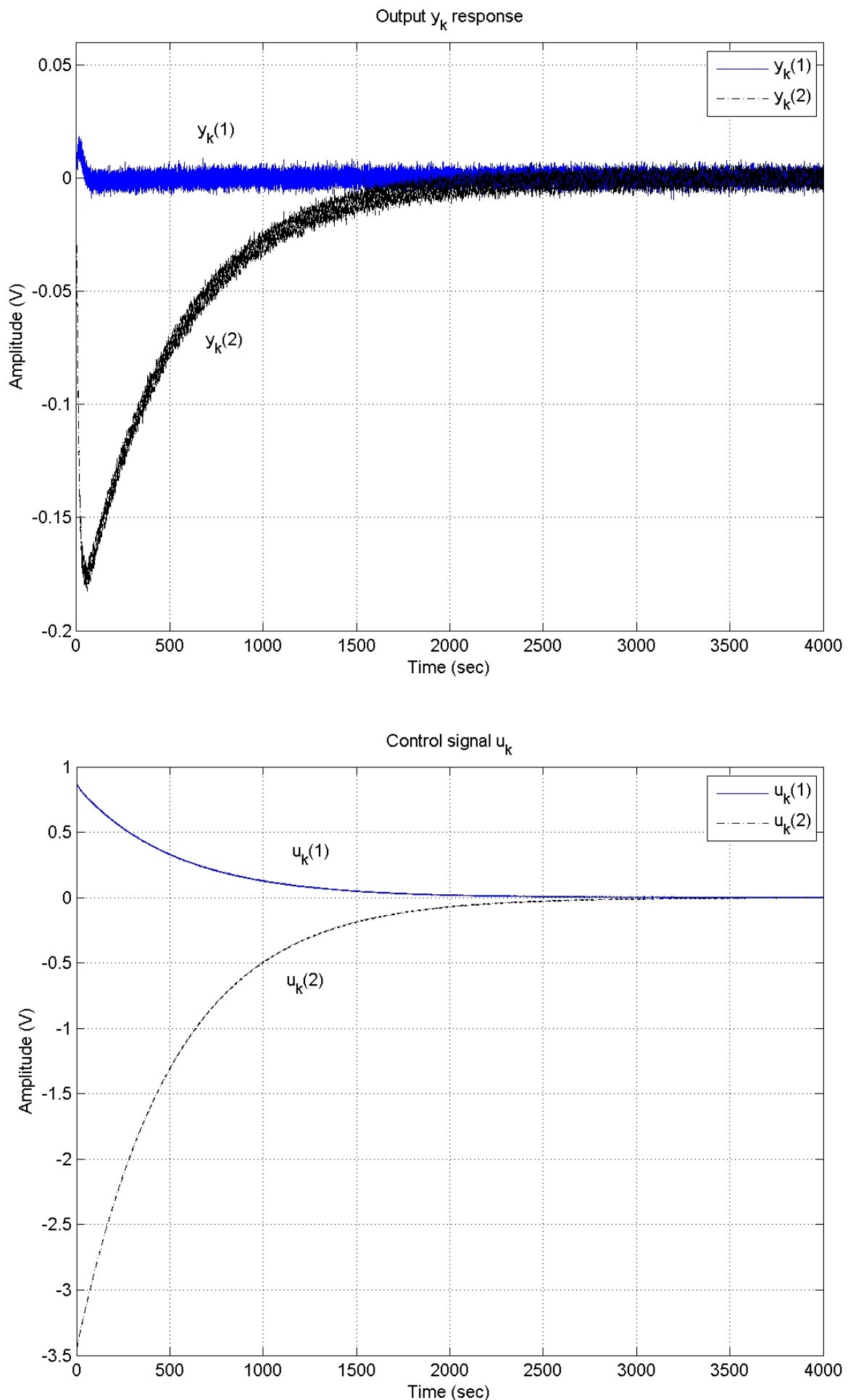

Figure 4.6-4: MARTS Example with Noise, Closed-Loop Response

Two-Term Controller (2.6) with Controller Gains (4.5)

Input $\eta_{0}=\left[\begin{array}{ll}0 & 100\end{array}\right]^{\top}$

Design 2 with Noise. 
Design 2 with Noise:

\begin{tabular}{|c|c|c|c|c|c|}
\hline \multicolumn{6}{|c|}{ Algorithm run \# 1} \\
\hline Starting $\mathrm{KP}$ & Starting & $\mathrm{KI}$ & theta & $\mathrm{mu}$ & Duration $\mathrm{T}$ (sec) \\
\hline 0.0005 & \multicolumn{2}{|c|}{$1 e-005$} & 0 & 1000 & 14000 \\
\hline Iteration & \multicolumn{2}{|l|}{ Func-count } & \multicolumn{2}{|l|}{$\min f(x)$} & Procedure \\
\hline 0 & 4 & \multicolumn{3}{|c|}{$5.02799 e+006$} & \multirow[b]{2}{*}{ initial simplex } \\
\hline 1 & 12 & \multicolumn{3}{|c|}{$4.89226 e+006$} & \\
\hline 2 & 20 & \multicolumn{3}{|c|}{$4.77073 e+006$} & expand \\
\hline 3 & 28 & \multicolumn{3}{|c|}{$4.55873 e+006$} & expand \\
\hline 4 & 36 & \multicolumn{3}{|c|}{$4.29557 e+006$} & expand \\
\hline 5 & 44 & \multicolumn{3}{|c|}{$3.94579 e+006$} & expand \\
\hline 6 & 52 & \multicolumn{3}{|c|}{$3.64872 e+006$} & expand \\
\hline 7 & 60 & \multicolumn{3}{|c|}{$3.56664 e+006$} & expand \\
\hline 8 & 68 & \multicolumn{3}{|c|}{$3.56664 e+006$} & contract \\
\hline 9 & 76 & \multicolumn{3}{|c|}{$3.55465 e+006$} & contract \\
\hline 10 & 84 & \multicolumn{3}{|c|}{$3.55428 e+006$} & reflect \\
\hline 11 & 92 & \multicolumn{3}{|c|}{$3.54923 e+006$} & contract \\
\hline 12 & 100 & \multicolumn{3}{|c|}{$3.54857 e+006$} & reflect \\
\hline 13 & 116 & \multicolumn{3}{|c|}{$3.54764 e+006$} & shrink \\
\hline 14 & 124 & \multicolumn{3}{|c|}{$3.54764 e+006$} & contract \\
\hline 15 & 140 & \multicolumn{3}{|c|}{$3.54764 e+006$} & shrink \\
\hline 16 & 144 & \multicolumn{3}{|c|}{$3.54764 e+006$} & reflect \\
\hline 17 & 152 & \multicolumn{3}{|c|}{$3.54623 e+006$} & contract \\
\hline 18 & 168 & 3.5 & $4623 e+006$ & & shrink \\
\hline 19 & 176 & 3.5 & $4623 e+006$ & & contract \\
\hline 20 & 192 & 3.5 & $4623 e+006$ & & shrink \\
\hline 21 & 196 & 3.5 & $4623 e+006$ & & reflect \\
\hline 22 & 204 & 3.5 & $4623 e+006$ & & contract \\
\hline 23 & 220 & 3.5 & $4623 e+006$ & & shrink \\
\hline 24 & 236 & 3.5 & $4623 e+006$ & & shrink \\
\hline 25 & 240 & 3.5 & $4623 e+006$ & & reflect \\
\hline 26 & 248 & 3.5 & $4613 e+006$ & & contract \\
\hline 27 & 264 & 3.5 & $4613 e+006$ & & shrink \\
\hline 28 & 272 & 3.5 & $4613 e+006$ & & contract \\
\hline 29 & 288 & 3.5 & $4613 e+006$ & & shrink \\
\hline 30 & 292 & 3.5 & $4613 e+006$ & & reflect \\
\hline 31 & 308 & 3.5 & $4613 e+006$ & & shrink \\
\hline Optimal KP & Optimal & $\mathrm{KI}$ & Optimal & cost $\mathrm{J}$ & \\
\hline 0.021107 & 0.0009314 & 408 & 3.546 & $13 e+006$ & \\
\hline Algorithm run & n 2 & & & & \\
\hline Starting KP & Starting & KI & theta & $\mathrm{mu}$ & Duration $\mathrm{T}$ (sec) \\
\hline 0.021107 & 0.000931408 & & 0 & 1000 & 5000 \\
\hline Iteration & Func-count & & $\min f(x)$ & & Procedure \\
\hline 0 & 4 & 3.5 & $4784 e+006$ & & \\
\hline 1 & 12 & 3.5 & $4784 e+006$ & & initial simplex \\
\hline 2 & 20 & 3.5 & $4784 e+006$ & & contract \\
\hline
\end{tabular}




\begin{tabular}{|c|c|c|c|}
\hline 3 & 28 & $3.54784 e+006$ & contract \\
\hline 4 & 36 & $3.54784 e+006$ & contract \\
\hline 5 & 44 & $3.54784 e+006$ & contract \\
\hline 6 & 52 & $3.54706 e+006$ & contract \\
\hline 7 & 60 & $3.54578 e+006$ & contract \\
\hline 8 & 76 & $3.54578 e+006$ & shrink \\
\hline 9 & 92 & $3.54578 e+006$ & shrink \\
\hline 10 & 100 & $3.54578 e+006$ & contract \\
\hline 11 & 116 & $3.54578 e+006$ & shrink \\
\hline 12 & 132 & $3.54547 e+006$ & shrink \\
\hline 13 & 148 & $3.54547 e+006$ & shrink \\
\hline 14 & 152 & $3.54547 e+006$ & reflect \\
\hline 15 & 156 & $3.54547 e+006$ & reflect \\
\hline 16 & 172 & $3.54547 e+006$ & shrink \\
\hline 17 & 176 & $3.54547 e+006$ & reflect \\
\hline 18 & 184 & $3.54547 e+006$ & contract \\
\hline 19 & 200 & $3.54547 e+006$ & shrink \\
\hline 20 & 216 & $3.54547 e+006$ & shrink \\
\hline 21 & 232 & $3.54547 e+006$ & shrink \\
\hline 22 & 236 & $3.54547 e+006$ & reflect \\
\hline 23 & 244 & $3.54547 e+006$ & contract \\
\hline 24 & 252 & $3.54547 e+006$ & contract \\
\hline 25 & 260 & $3.54547 e+006$ & contract \\
\hline 26 & 276 & $3.54547 e+006$ & shrink \\
\hline 27 & 292 & $3.54547 e+006$ & shrink \\
\hline Optimal KP & Optimal K & Optimal cost $\mathrm{J}$ & \\
\hline 0.0281928 & 0.00094790 & $3.54547 e+006$ & \\
\hline
\end{tabular}




\subsection{Control Design 3 for MARTS}

This study shows that "high performance control" can be achieved by decreasing the $\mu$ parameter in the performance index $\left(J_{B} 2.103\right)$.

The tuning regulator (2.17) with $\epsilon=10^{-3}$ is first applied to the plant and the twoterm controller (2.6) is then obtained by minimizing the performance index $J_{B y_{0}}(2.78)$ using $\theta=0, \mu=1$. The optimization is carried out under the system settings of:

$$
y_{0}=\left[\begin{array}{c}
-0.1 \\
-0.15
\end{array}\right], \quad \eta_{0}=\left[\begin{array}{l}
0 \\
0
\end{array}\right] \text {, and } \quad y_{r e f}=\left[\begin{array}{l}
0 \\
0
\end{array}\right]
$$

in this case the optimal control gains $K_{P}$ and $K_{I}$ are found to be

$$
K_{P}=8.0229 \times 10^{-1}, \quad K_{I}=6.3518 \times 10^{-2} .
$$

Simulation of plant's initial condition input and tracking reference input is given in Figures 4.7-1 to 4.7-8 for the resulting closed-loop system.

Remark 4.11. For the tuning regulator, the initial peak value of $u_{k}$ decreases as the gain $\epsilon$ is made smaller.

Remark 4.12. In this example, the controller with gains (4.6) is optimal only for the initial condition $y_{0}=\left[\begin{array}{ll}-0.1 & -0.15\end{array}\right]^{\top}$. The "averaging" technique described in Section 2.7 was not applied. 

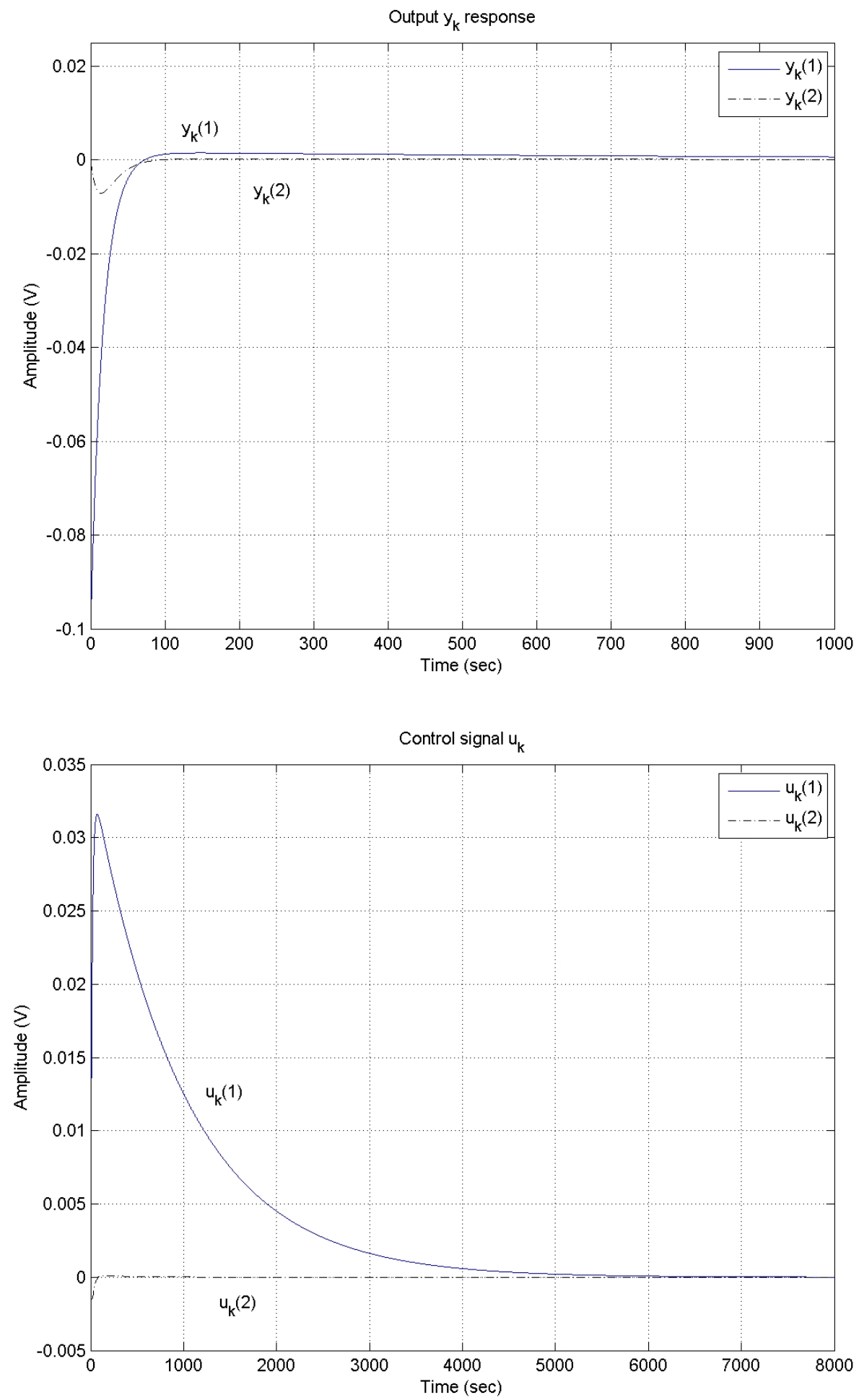

Figure 4.7-1: MARTS Example, Closed-Loop Response

Tuning Regulator (2.17) with $\epsilon=10^{-3}$

Input $y_{0}=\left[\begin{array}{ll}-0.1 & 0\end{array}\right]^{\top}$

Design 3. 

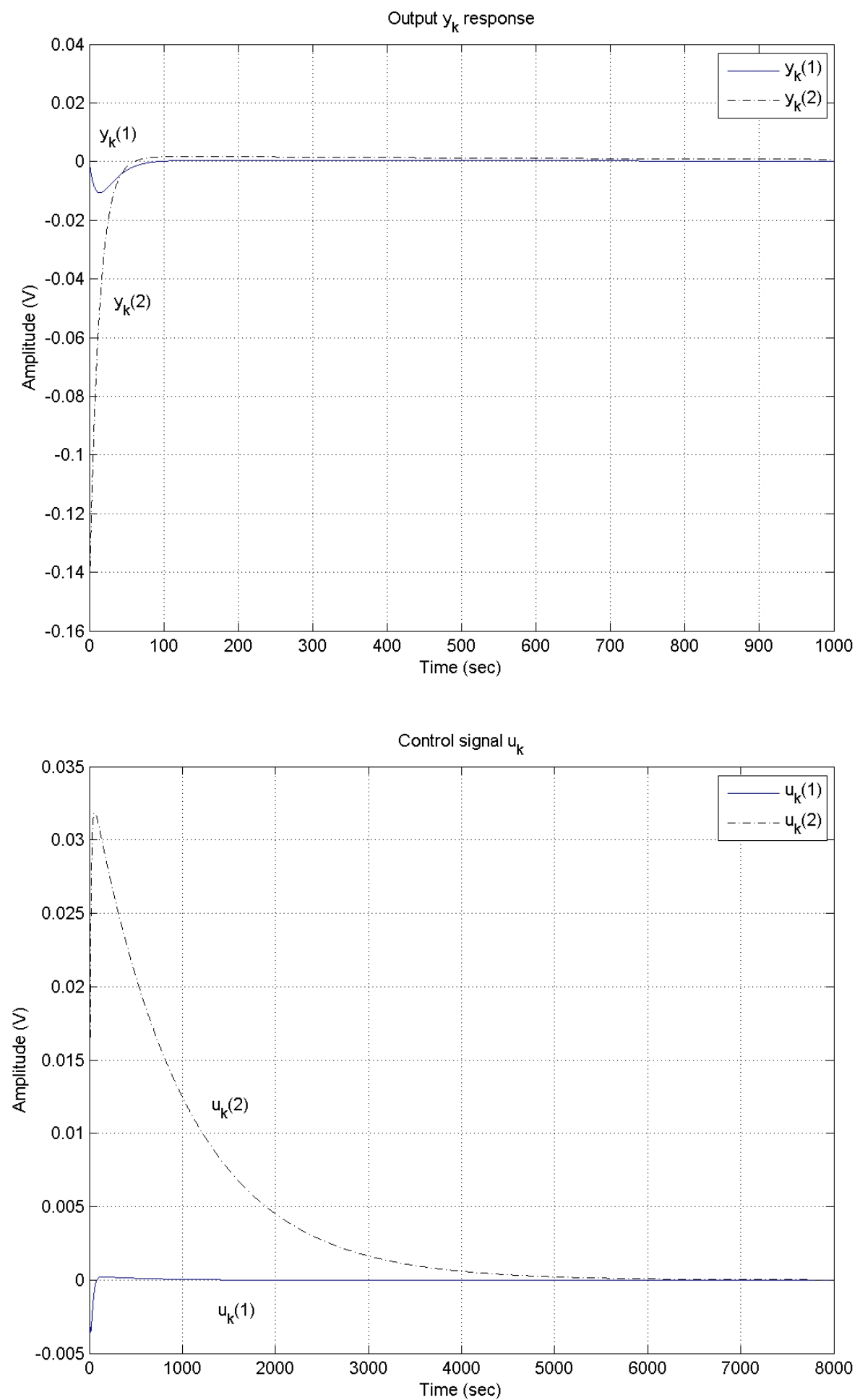

Figure 4.7-2: MARTS Example, Closed-Loop Response

Tuning Regulator (2.17) with $\epsilon=10^{-3}$

Input $y_{0}=\left[\begin{array}{ll}0 & -0.15\end{array}\right]^{\top}$

Design 3. 

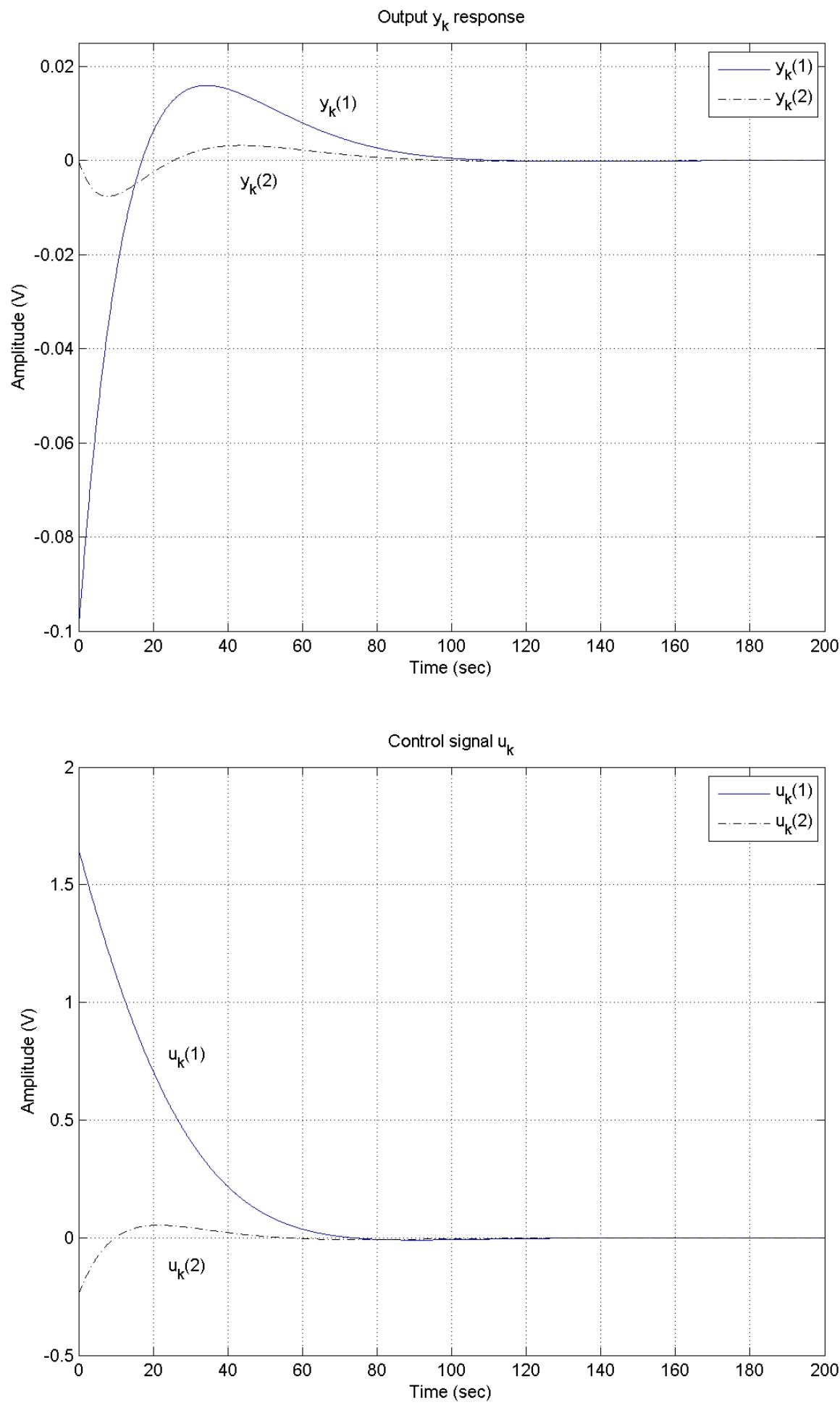

Figure 4.7-3: MARTS Example, Closed-Loop Response Two-Term Controller (2.6) with Controller Gains (4.6) Input $y_{0}=\left[\begin{array}{ll}-0.1 & 0\end{array}\right]^{\top}$ Design 3. 

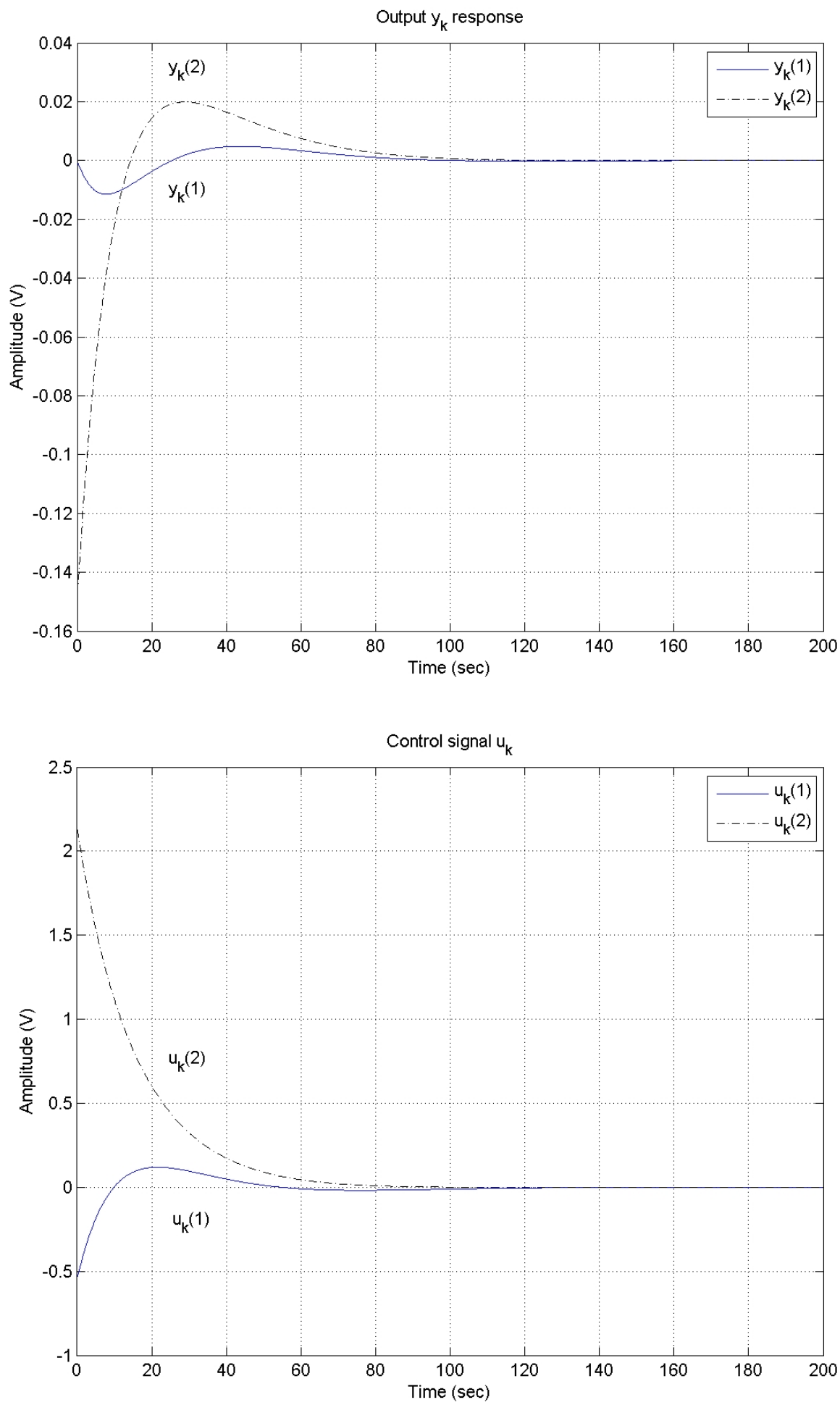

Figure 4.7-4: MARTS Example, Closed-Loop Response Two-Term Controller (2.6) with Controller Gains (4.6) Input $y_{0}=\left[\begin{array}{ll}0 & -0.15\end{array}\right]^{\top}$

Design 3. 

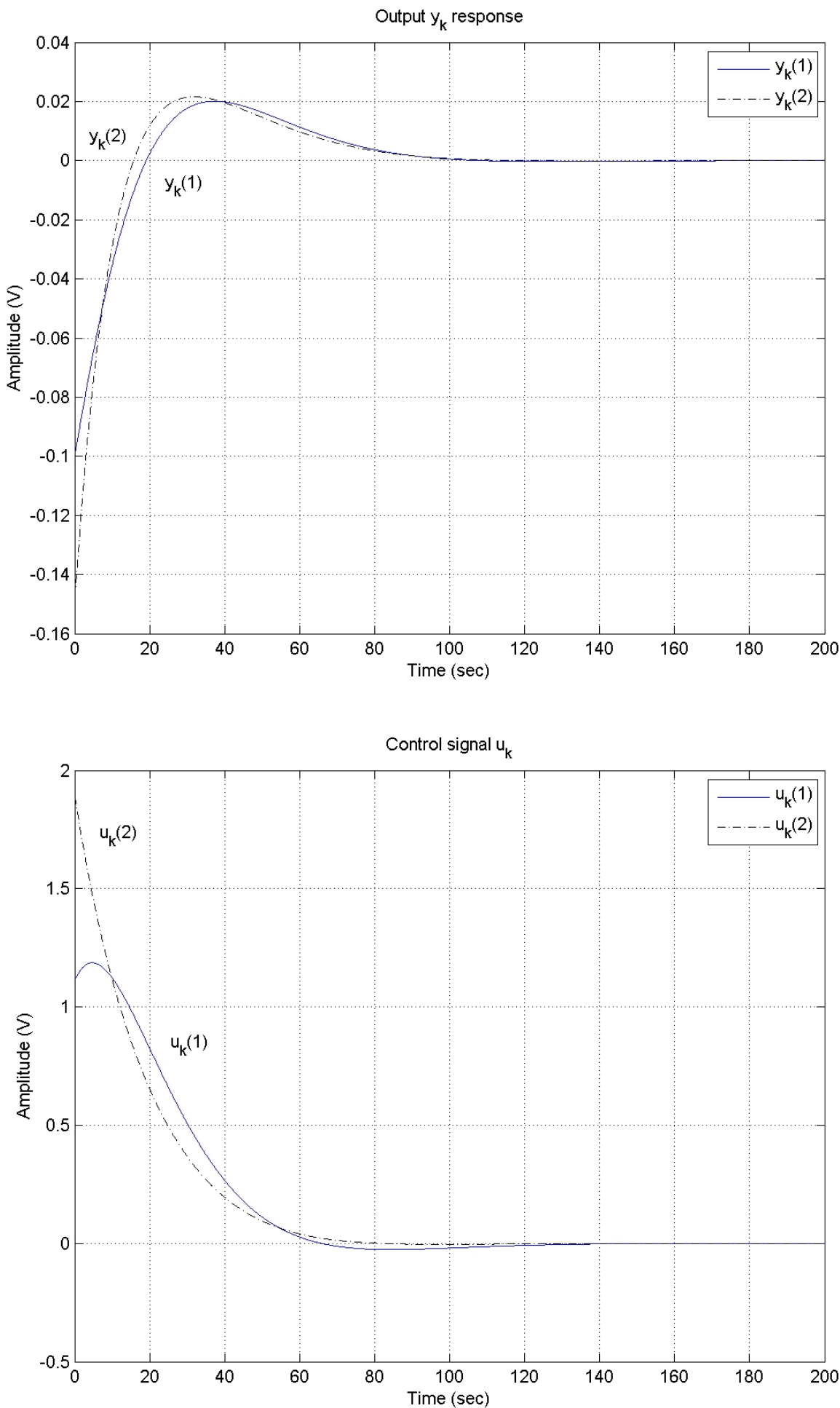

Figure 4.7-5: MARTS Example, Closed-Loop Response Two-Term Controller (2.6) with Controller Gains (4.6)

Input $y_{0}=\left[\begin{array}{ll}-0.1 & -0.15\end{array}\right]^{\top}$

Design 3. 

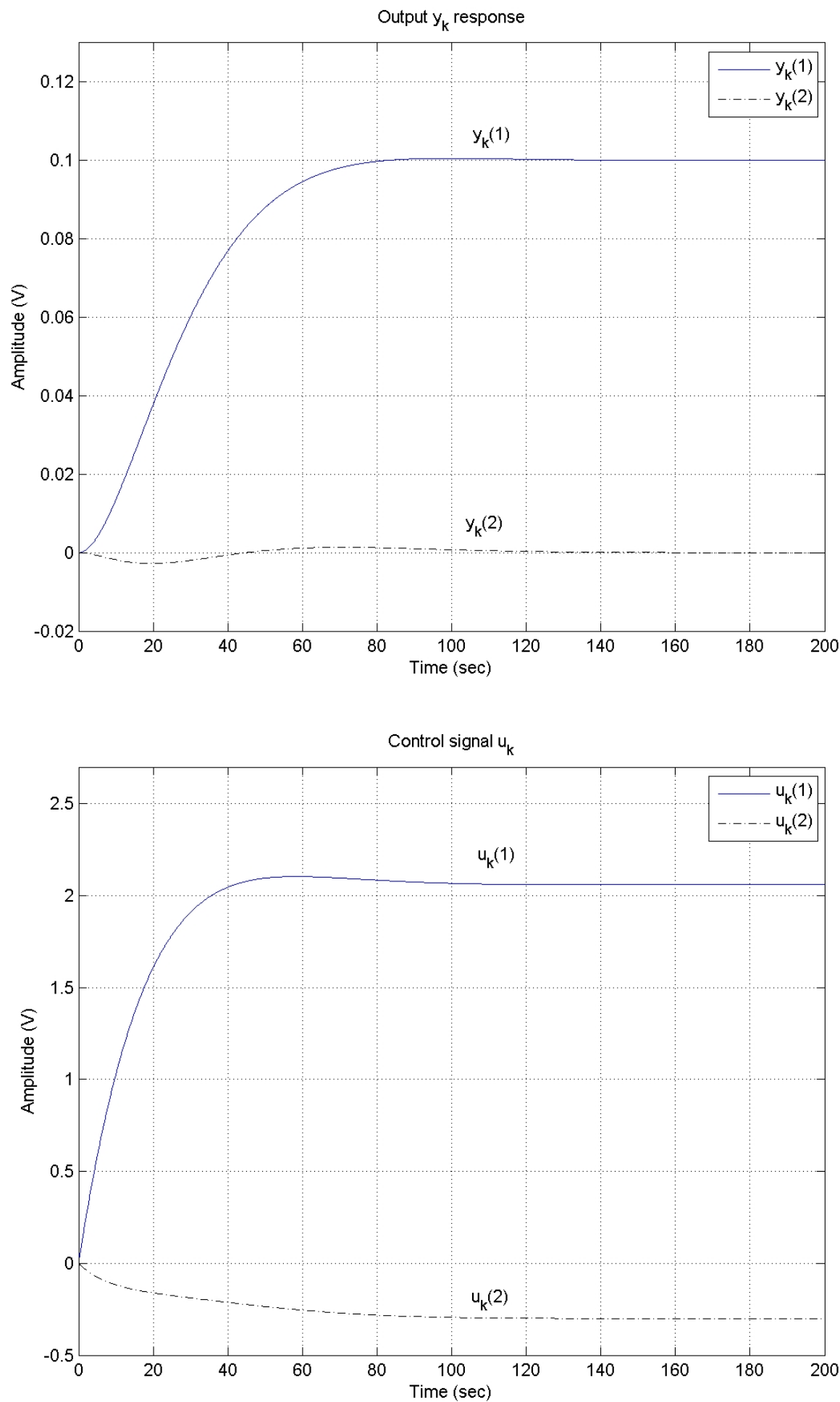

Figure 4.7-6: MARTS Example, Closed-Loop Response Two-Term Controller (2.6) with Controller Gains (4.6) Input $y_{\text {ref }}=\left[\begin{array}{ll}0.1 & 0\end{array}\right]^{\top}$ Design 3. 

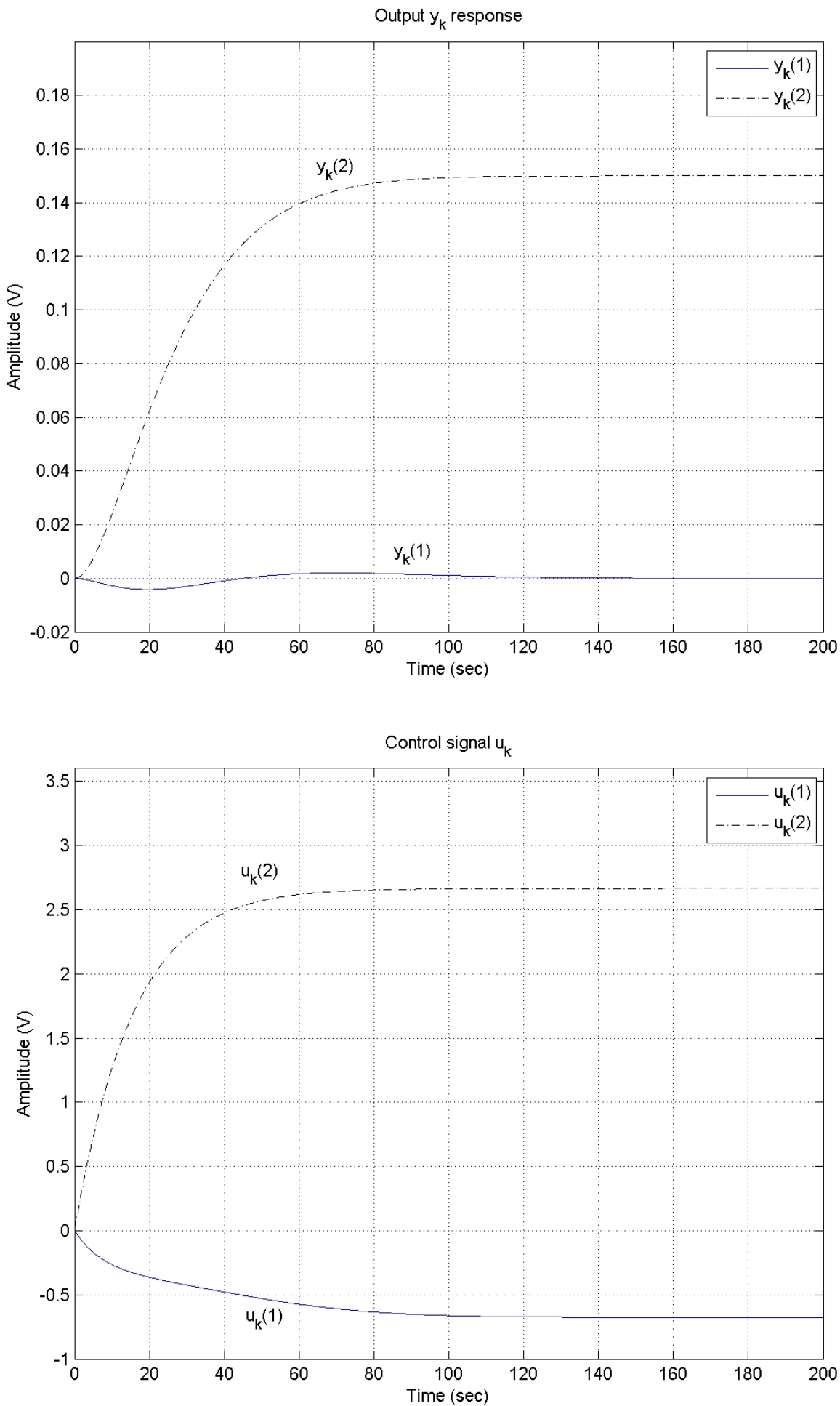

Figure 4.7-7: MARTS Example, Closed-Loop Response Two-Term Controller (2.6) with Controller Gains (4.6) Input $y_{\text {ref }}=\left[\begin{array}{ll}0 & 0.15\end{array}\right]^{\top}$

Design 3. 

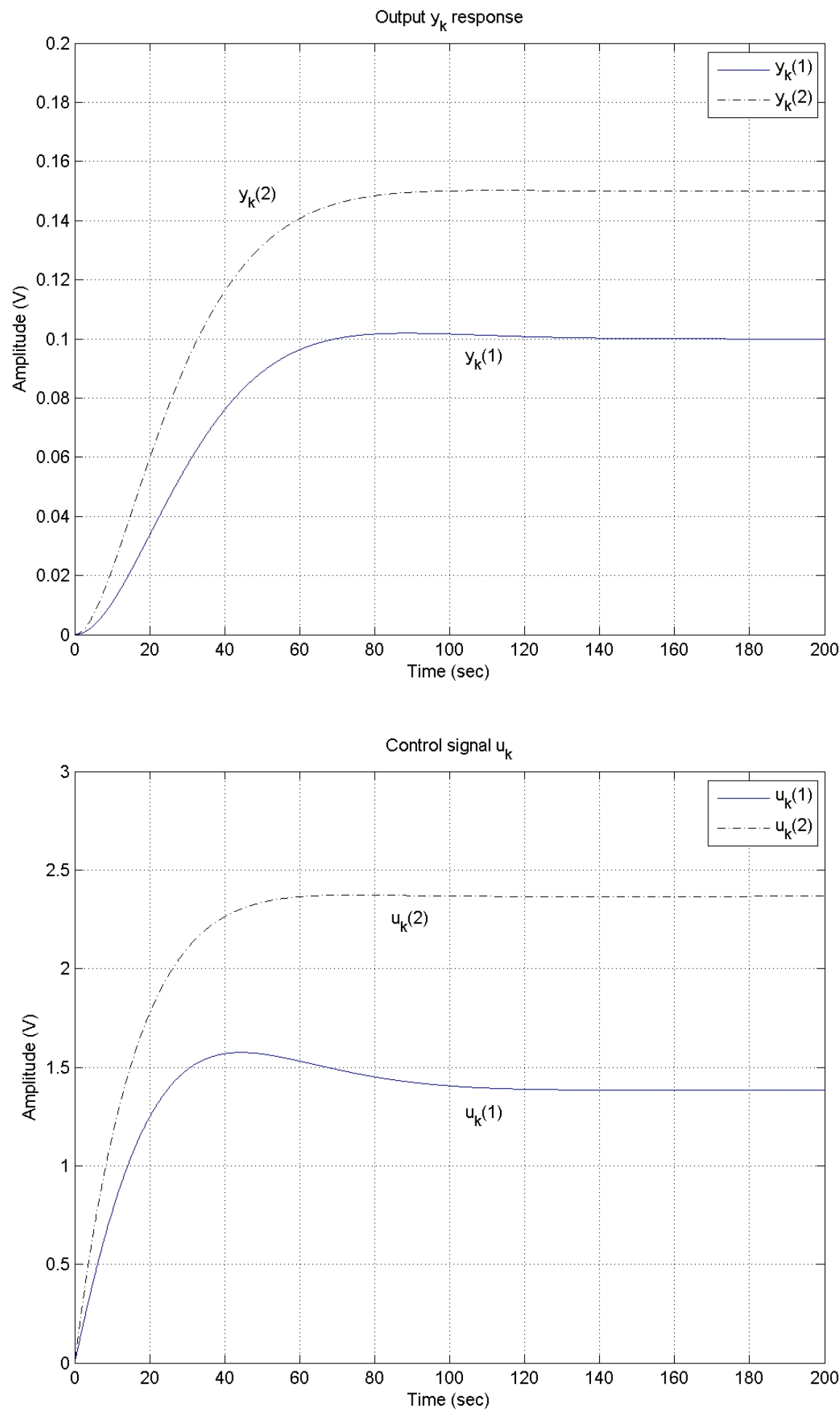

Figure 4.7-8: MARTS Example, Closed-Loop Response Two-Term Controller (2.6) with Controller Gains (4.6) Input $y_{\text {ref }}=\left[\begin{array}{ll}0.1 & 0.15\end{array}\right]^{\top}$

Design 3. 


\section{Design 3:}

\begin{tabular}{|c|c|c|c|c|}
\hline \multicolumn{5}{|c|}{ Algorithm run \# 1} \\
\hline $\begin{array}{c}\text { Starting KP } \\
0.0005\end{array}$ & $\begin{array}{l}\text { Starting KI } \\
\text { 1e-005 }\end{array}$ & $\begin{array}{c}\text { theta } \\
0\end{array}$ & $\begin{array}{r}\mathrm{mu} \\
1\end{array}$ & $\begin{array}{r}\text { Duration } \mathrm{T} \text { (sec) } \\
14000\end{array}$ \\
\hline Iteration & Func-count & $\min f(x)$ & & Procedure \\
\hline 0 & 1 & 4681.85 & & \\
\hline 1 & 3 & 4671.31 & & initial simplex \\
\hline 2 & 5 & 4657.62 & & expand \\
\hline 3 & 7 & 4641.18 & & expand \\
\hline 4 & 9 & 4606.12 & & expand \\
\hline 5 & 11 & 4556.95 & & expand \\
\hline 6 & 13 & 4466.23 & & expand \\
\hline 7 & 15 & 4332.2 & & expand \\
\hline 8 & 17 & 4110.83 & & expand \\
\hline 9 & 19 & 3795.87 & & expand \\
\hline 10 & 21 & 3346.87 & & expand \\
\hline 11 & 23 & 2794.83 & & expand \\
\hline 12 & 25 & 2169.21 & & expand \\
\hline 13 & 27 & 1566.05 & & expand \\
\hline 14 & 29 & 1045.47 & & expand \\
\hline 15 & 31 & 653.869 & & expand \\
\hline 16 & 33 & 385.277 & & expand \\
\hline 17 & 35 & 222.244 & & expand \\
\hline 18 & 37 & 138.48 & & expand \\
\hline 19 & 39 & 115.815 & & expand \\
\hline 20 & 40 & 115.815 & & reflect \\
\hline 21 & 42 & 115.815 & & contract \\
\hline 22 & 46 & 115.8 & & shrink \\
\hline 23 & 50 & 115.679 & & shrink \\
\hline 24 & 52 & 115.679 & & contract \\
\hline 25 & 54 & 115.679 & & contract \\
\hline 26 & 58 & 115.679 & & shrink \\
\hline 27 & 62 & 115.679 & & shrink \\
\hline 28 & 64 & 115.679 & & contract \\
\hline 29 & 66 & 115.676 & & expand \\
\hline 30 & 67 & 115.676 & & reflect \\
\hline 31 & 69 & 115.672 & & expand \\
\hline 32 & 70 & 115.672 & & reflect \\
\hline 33 & 72 & 115.669 & & expand \\
\hline 34 & 74 & 115.657 & & expand \\
\hline 35 & 76 & 115.655 & & expand \\
\hline 36 & 78 & 115.62 & & expand \\
\hline 37 & 79 & 115.62 & & reflect \\
\hline 38 & 81 & 115.554 & & expand \\
\hline 39 & 82 & 115.554 & & reflect \\
\hline 40 & 84 & 115.426 & & expand \\
\hline 41 & 86 & 115.37 & & expand \\
\hline 42 & 88 & 115.063 & & expand \\
\hline 43 & 90 & 114.911 & & expand \\
\hline 44 & 92 & 114.196 & & expand \\
\hline
\end{tabular}




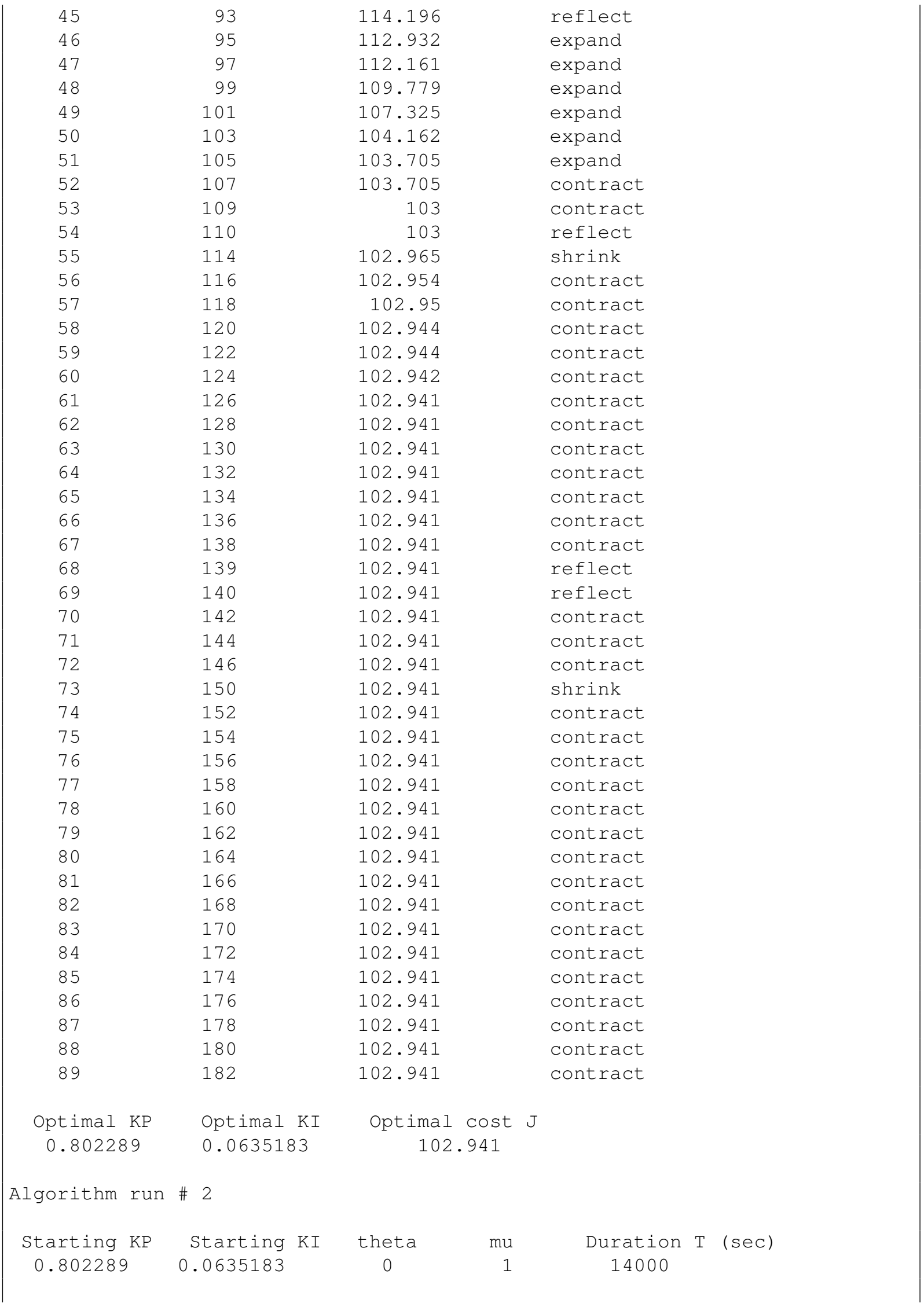




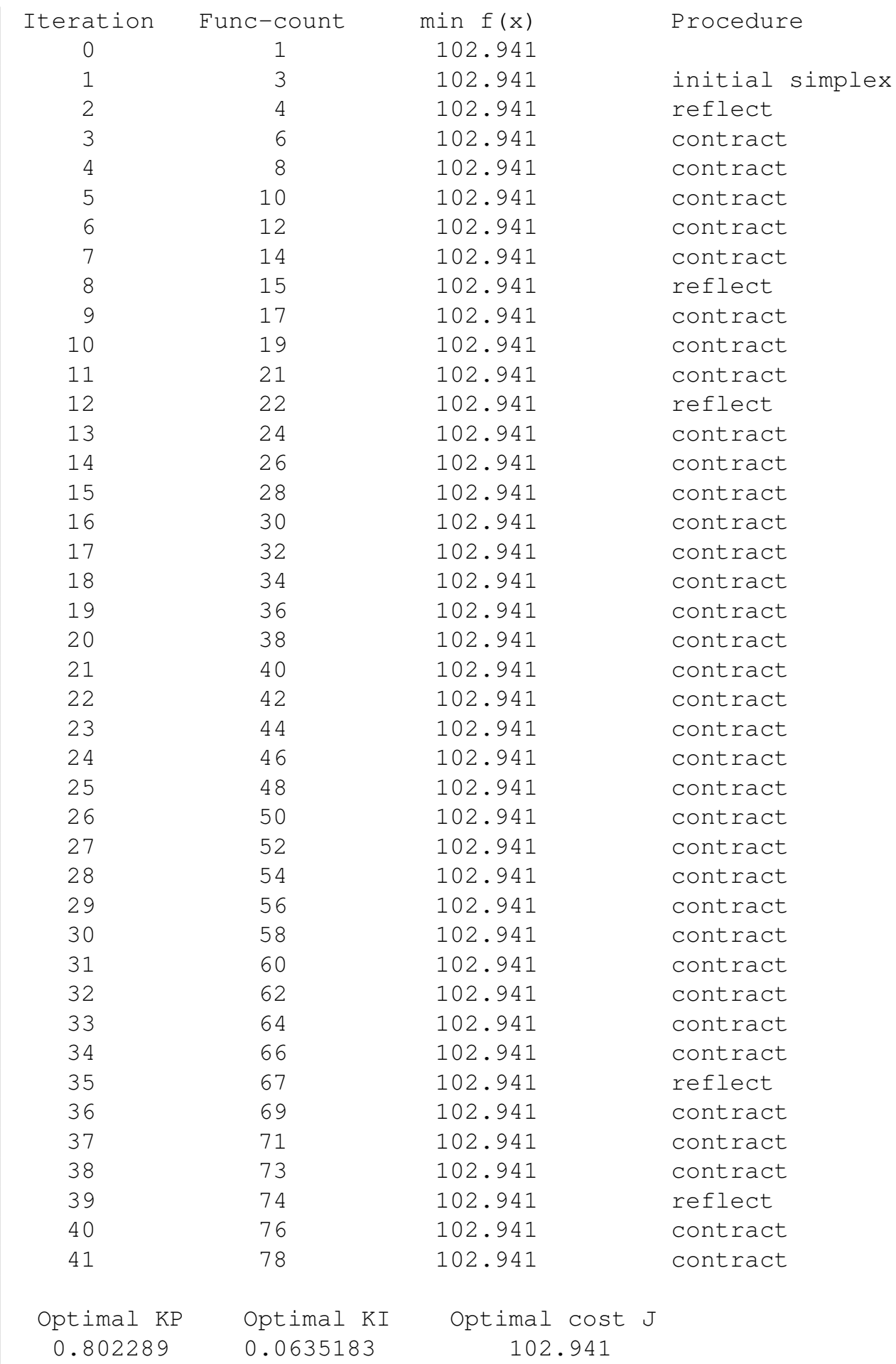




\subsection{Summary}

The proposed controller design approach is applied to a small-scale system that is openloop stable and minimum phase. A tuning regulator with a sufficiently small gain $\epsilon$ ensures that the control input $u_{k}$ monotonically approaches to the steady state value $u_{\infty}$ with no overshoot. Furthermore, as $\epsilon$ decreases, "peaking" in the control input $u_{k}$ diminishes. Solving either optimization problem A or B gives rise to the same optimal controller, but the performance index $J_{B}$ (2.103) is less sensitive to noise and can be minimized without disturbing any of the plant's settings. 


\section{Chapter 5}

\section{Williams and Otto Chemical Plant Example (Minimum Phase System)}

\subsection{Description of the Williams and Otto Chemical Plant}

The Williams and Otto chemical plant in [40] is described by a $41^{\text {st }}$ order model with 4 inputs, 4 outputs, and 3 disturbance input channels. The continuous plant model is given in Appendix B. The eigenvalues and transmission zeros of this continuous system are given by

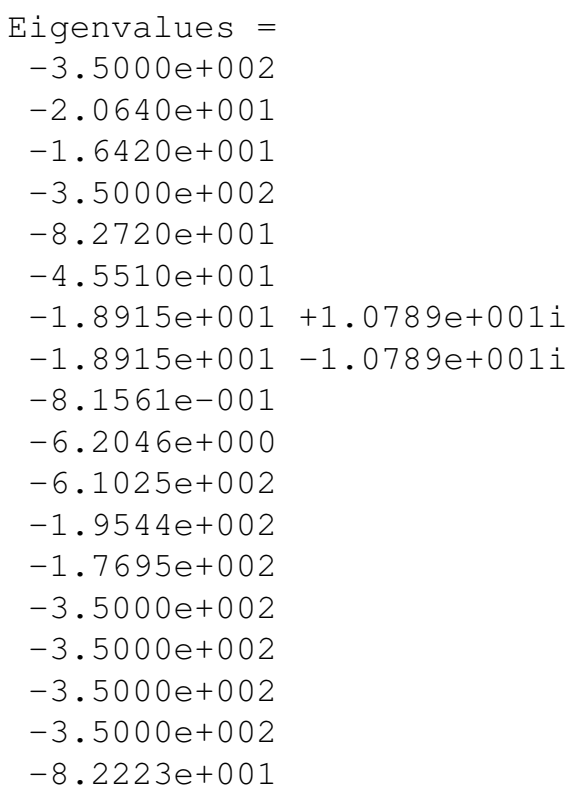

Eigenvalues $=$

$-3.5000 e+002$

$-2.0640 e+001$

$-1.6420 e+001$

$-3.5000 e+002$

$-8.2720 e+001$

$-4.5510 e+001$

$-1.8915 e+001+1.0789 e+001 i$

$-8.1561 e-001$

$-6.2046 e+000$

$-6.1025 e+002$

$-1.9544 e+002$

$-1.7695 e+002$

$-3.5000 e+002$

$-3.5000 e+002$

$-8.2223 e+001$

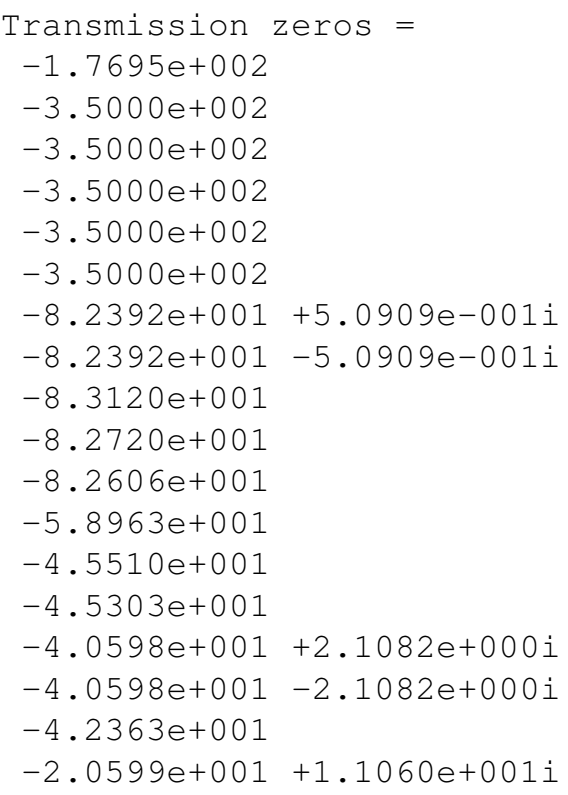




$-8.3146 e+001$
$-8.2607 e+001$
$-8.2688 e+001$
$-5.8594 e+001$
$-4.6270 e+001$
$-4.5125 e+001$
$-4.1266 e+001$
$-3.5820 e+001$
$-3.5197 e+001$
$-1.8873 e+001+1.0987 e+001 i$
$-1.8873 e+001-1.0987 e+001 i$
$-2.1160 e+001+9.9877 e+000 i$
$-2.1160 e+001-9.9877 e+000 i$
$-2.0584 e+001$
$-1.5720 e+001$
$-9.7921 e+000$
$-7.6450 e+000$
$-6.7738 e+000$
$-4.9051 e+000$
$-3.3063 e-001$
$-6.0560 e-001$
$-9.9564 e-001+3.9048 e-002 i$
$-9.9564 e-001-3.9048 e-002 i$

$-2.0599 e+001-1.1060 e+001 i$

$-1.8885 e+001+1.1275 e+001 i$

$-1.8885 e+001-1.1275 e+001 i$

$-1.8915 e+001+1.0789 e+001 i$

$-1.8915 e+001-1.0789 e+001 i$

$-1.9842 e+001$

$-1.5695 e+001$

$-9.5391 e+000+3.8361 e-001 i$

$-9.5391 e+000-3.8361 e-001 i$

$-6.2046 e+000$

$-5.1118 e+000$

$-1.7432 e+000+1.9494 e+000 i$

$-1.7432 e+000-1.9494 e+000 i$

$-4.2012 e-001$

$-9.5316 e-001$

$-8.1561 e-001$

$-2.0640 e+001$

$-3.5000 e+002$

If we sample the system with $h=0.01$, the resultant sampled system is open-loop stable and minimum phase with eigenvalues and transmission zeros given by

Eigenvalues $=$
$3.0197 e-002$
$8.1351 e-001$
$8.4857 e-001$
$4.3727 e-001$
$2.2372 e-003$
$6.3438 e-001$
$1.4165 e-001$
$1.7041 e-001$
$8.2285 e-001+8.9127 e-002 i$
$8.2285 e-001-8.9127 e-002 i$
$9.3984 e-001$
$9.9188 e-001$
$9.9670 e-001$
$9.9396 e-001$
$9.9009 e-001+3.8661 e-004 i$
$9.9009 e-001-3.8661 e-004 i$
$9.5213 e-001$
$9.3450 e-001$
$9.2640 e-001$
$9.0672 e-001$
$8.2301 e-001+9.0787 e-002 i$
$8.2301 e-001-9.0787 e-002 i$
$8.0525 e-001+8.0695 e-002 i$
$8.0525 e-001-8.0695 e-002 i$
$8.5453 e-001$

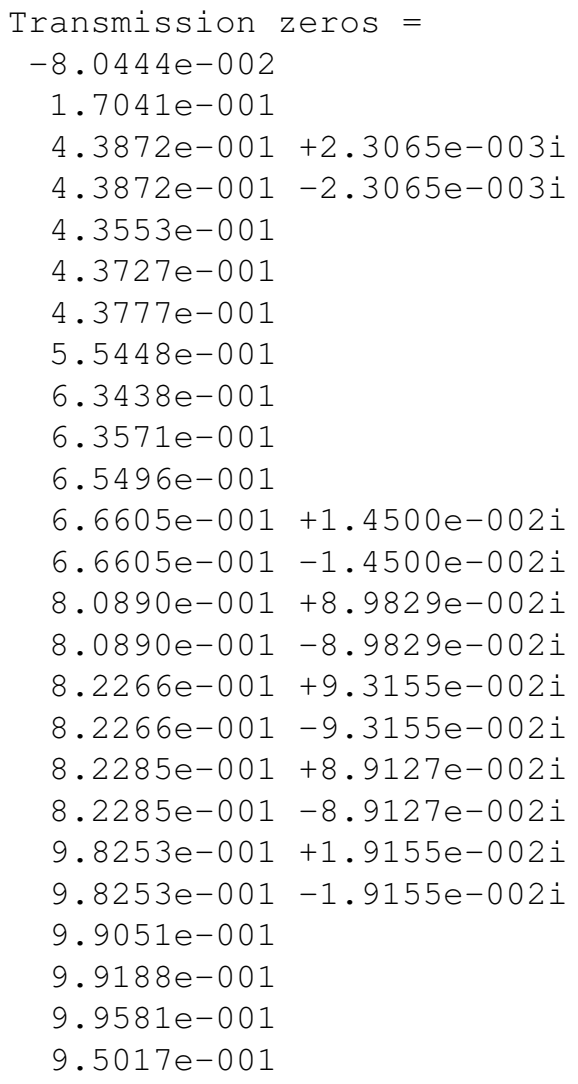


$8.1396 e-001$

$5.5658 e-001$

$7.0330 e-001$

$6.9893 e-001$

$6.6189 e-001$

$6.2958 e-001$

$6.3683 e-001$

$4.3945 e-001$

$4.3541 e-001$

$4.3777 e-001$

$4.3741 e-001$

$3.0197 e-002$

$3.0197 e-002$

$3.0197 e-002$

$3.0197 e-002$

$3.0197 e-002$
$9.3984 e-001$

$9.0901 e-001+3.4893 e-003 i$

$9.0901 e-001-3.4893 e-003 i$

$8.5474 \mathrm{e}-001$

$8.2003 e-001$

$8.1351 e-001$

$3.0197 e-002$

$3.0197 e-002$

3.0197e-002

$3.0197 e-002$

$3.0197 e-002$

$3.0197 e-002$

It is to be noted that the mathematical model of the plant (B.1) is used in this chapter only to carry out simulations, but it is not used for controller design.

Since actuator constraints were not given in [40], we assume that the operating range of the control signals is given by

$$
\begin{gathered}
-5 \leq u_{k}(1) \leq 5 \\
-5 \leq u_{k}(2) \leq 5 \\
-5 \leq u_{k}(3) \leq 5 \\
-5 \leq u_{k}(4) \leq 5 .
\end{gathered}
$$

Open-loop responses of this system are given in Figures 5.1-1 to 5.1-4. The steady-state tracking matrix $\Gamma(2.2)$ is experimentally obtained to be:

$$
\Gamma=\left[\begin{array}{cccc}
9.38 \times 10^{-2} & 0 & 0 & -7.54 \times 10^{-4} \\
6.95 \times 10^{-2} & 2.59 \times 10^{-1} & 0 & -5.58 \times 10^{-4} \\
6.95 \times 10^{-2} & 2.59 \times 10^{-1} & 6.09 \times 10^{-4} & -5.58 \times 10^{-4} \\
0 & 0 & 0 & -1.00 \times 10^{-5}
\end{array}\right] .
$$



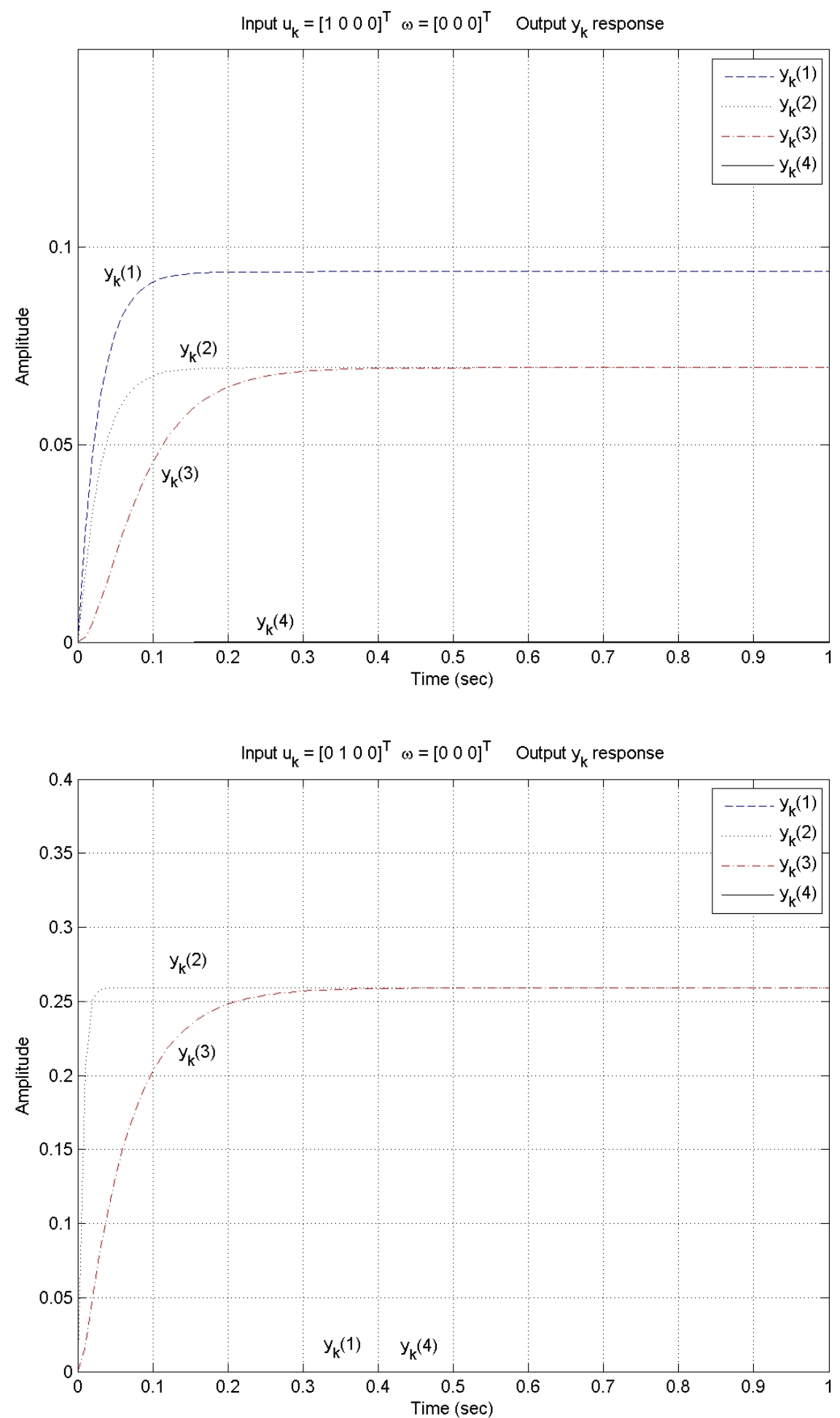

Figure 5.1-1: Williams and Otto Chemical Plant Example, Open-Loop Response. 

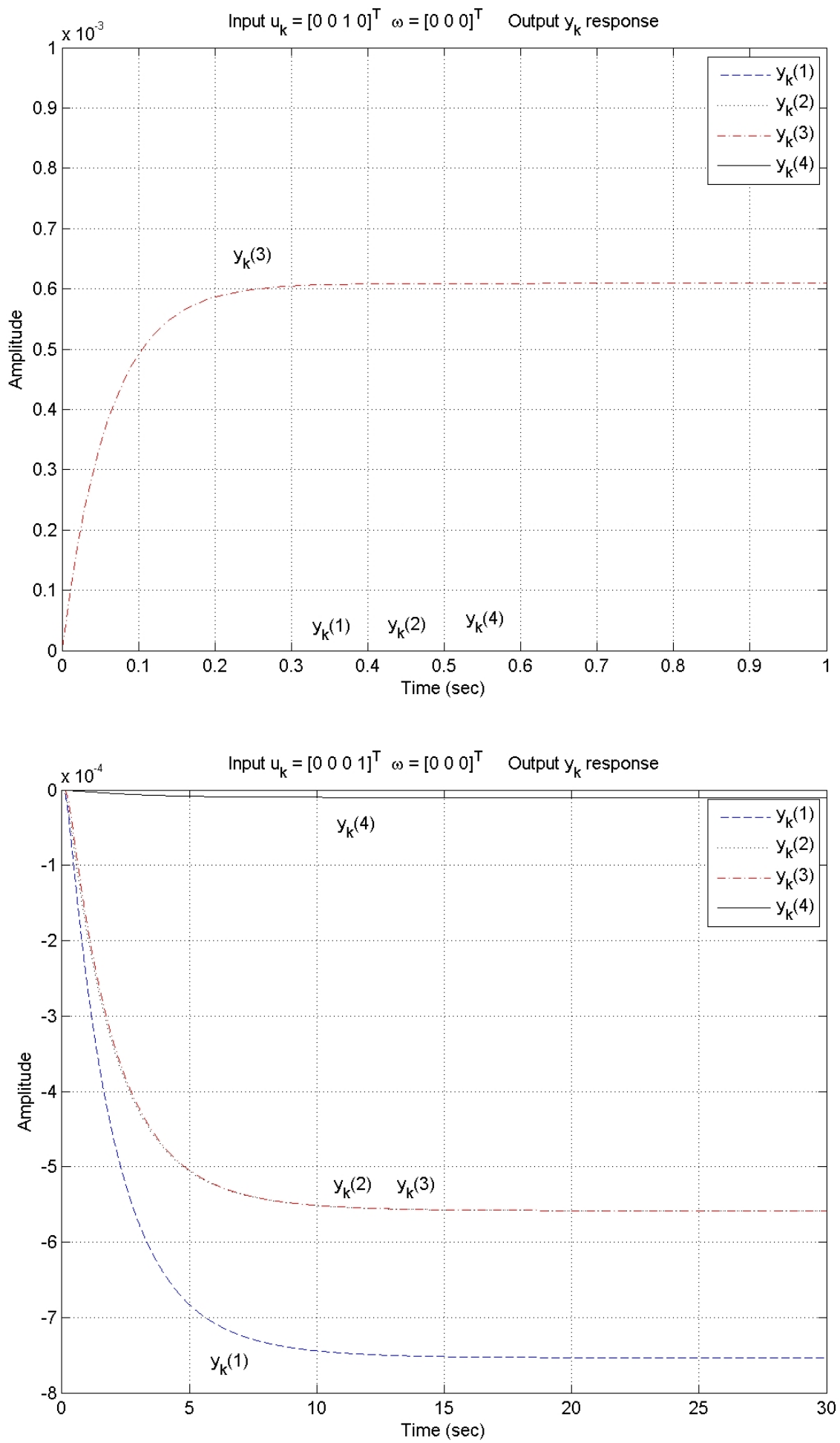

Figure 5.1-2: Williams and Otto Chemical Plant Example, Open-Loop Response. 

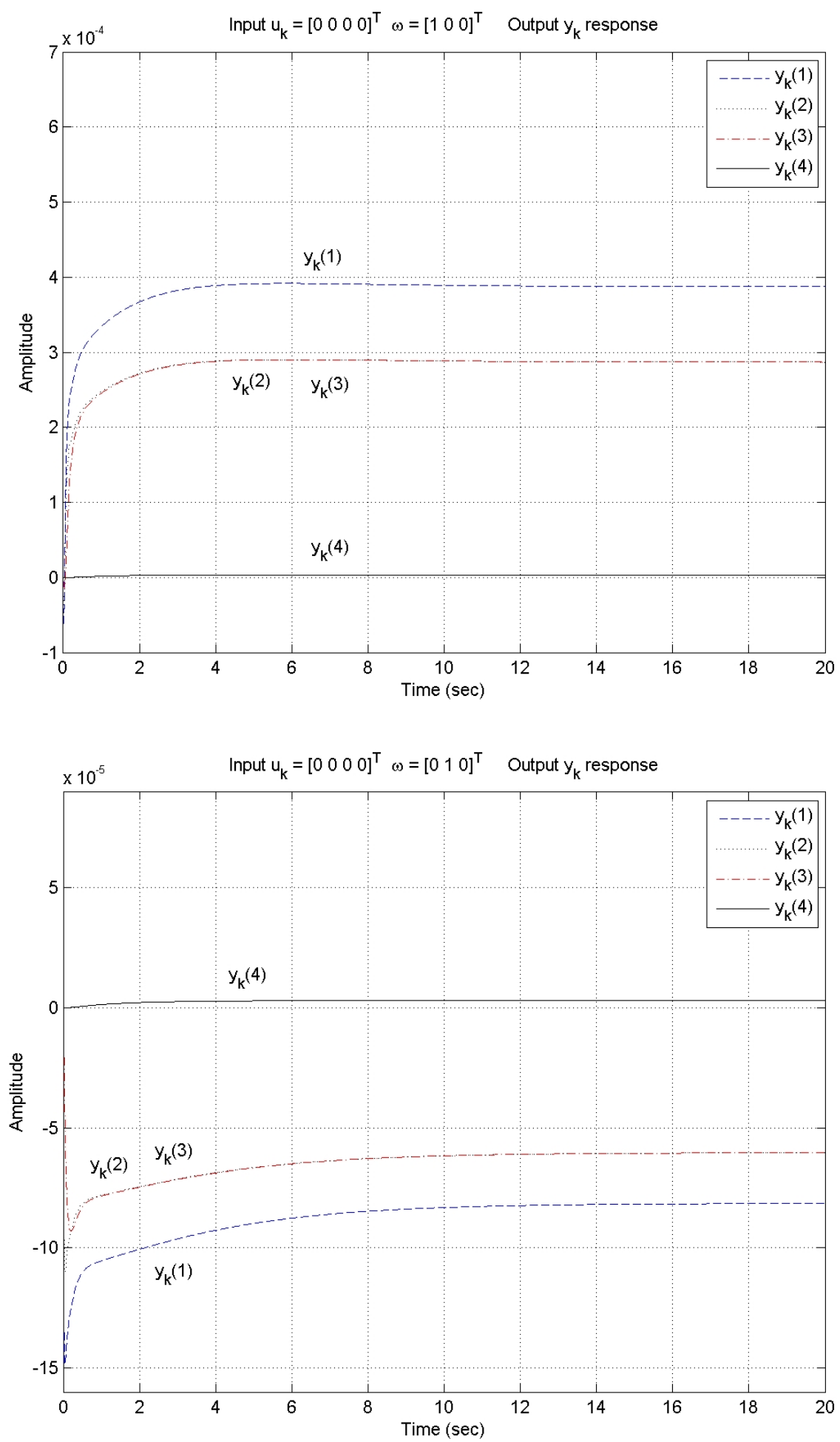

Figure 5.1-3: Williams and Otto Chemical Plant Example, Open-Loop Response. 


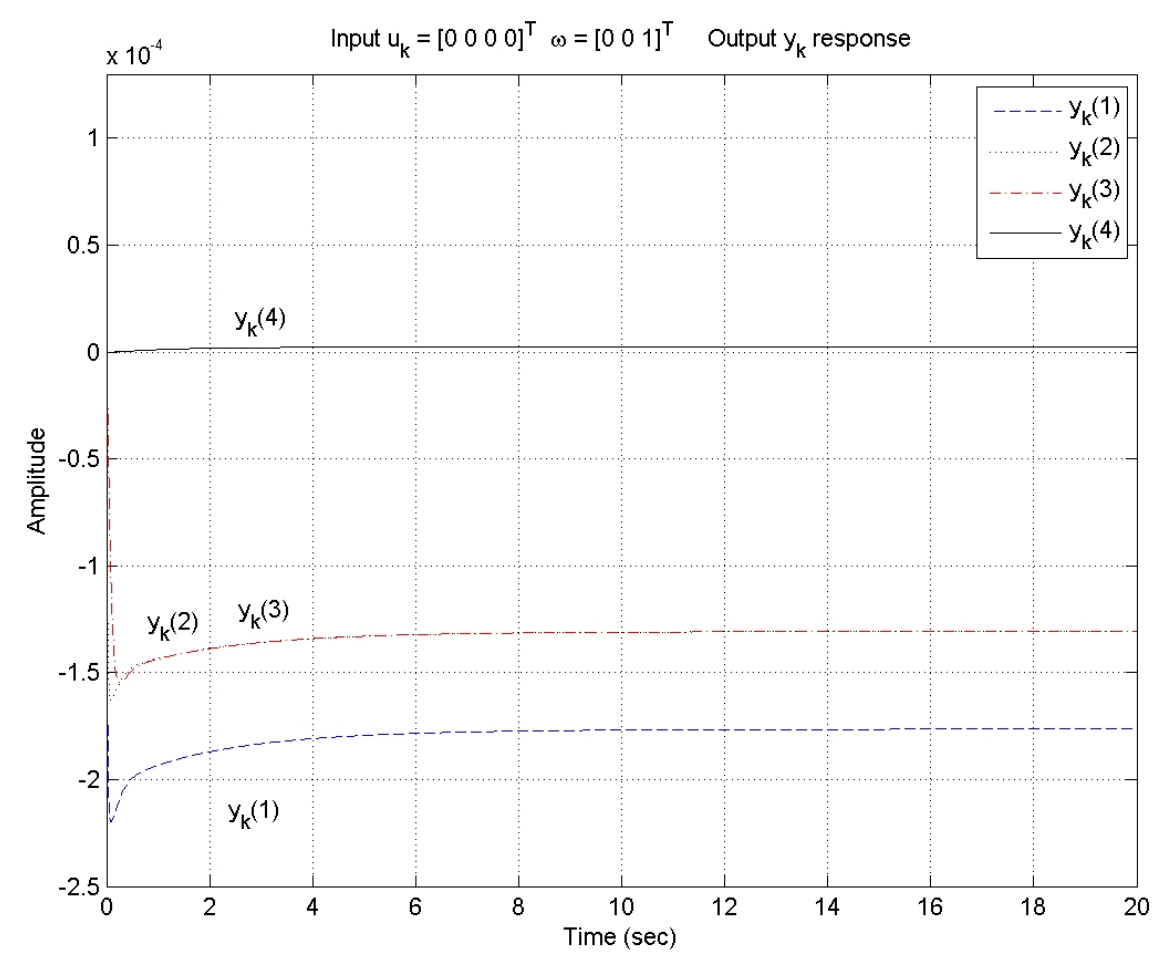

Figure 5.1-4: Williams and Otto Chemical Plant Example, Open-Loop Response.

\subsection{Control Design for Williams and Otto Chemical Plant}

In this study, it is desired to design a controller to provide disturbance rejection for the case of constant disturbances in $\omega(1), \omega(2), \omega(3)$. The tuning regulator (2.17) with $\epsilon=10^{-2}$ is first applied to the plant and the two-term controller (2.6) is then obtained by minimizing the performance index $J_{A \omega}$ (2.58) using $\theta=0, \mu=10^{-6}$. The optimization is carried out under the system settings of:

$$
\begin{gathered}
x_{0}=\left[\begin{array}{l}
0 \\
\vdots \\
0
\end{array}\right]_{41 \times 1}, \eta_{0}=\left[\begin{array}{c}
0 \\
\vdots \\
0
\end{array}\right]_{4 \times 1}, y_{r e f}=\left[\begin{array}{c}
0 \\
\vdots \\
0
\end{array}\right]_{4 \times 1}, \text { and } \\
\mathcal{Z}_{\omega}=\left\{\left[\begin{array}{l}
1 \\
0 \\
0
\end{array}\right],\left[\begin{array}{l}
0 \\
1 \\
0
\end{array}\right],\left[\begin{array}{l}
0 \\
0 \\
1
\end{array}\right],\left[\begin{array}{c}
\frac{1}{\sqrt{2}} \\
\frac{1}{\sqrt{2}} \\
0
\end{array}\right],\left[\begin{array}{c}
\frac{1}{\sqrt{2}} \\
-\frac{1}{\sqrt{2}} \\
0
\end{array}\right],\left[\begin{array}{c}
0 \\
\frac{1}{\sqrt{2}} \\
\frac{1}{\sqrt{2}}
\end{array}\right],\left[\begin{array}{c}
0 \\
\frac{1}{\sqrt{2}} \\
-\frac{1}{\sqrt{2}}
\end{array}\right],\left[\begin{array}{c}
\frac{1}{\sqrt{2}} \\
0 \\
\frac{1}{\sqrt{2}}
\end{array}\right],\left[\begin{array}{c}
\frac{1}{\sqrt{2}} \\
0 \\
-\frac{1}{\sqrt{2}}
\end{array}\right]\right\} .
\end{gathered}
$$


The above settings correspond to approximating the performance index $J_{A \omega}(2.58)$; in this case the optimal control gains $K_{P}$ and $K_{I}$ are found to be

$$
\text { Case } \theta=0: \quad K_{P}=8.7621 \times 10^{-2}, \quad K_{I}=6.1933 \times 10^{-1}
$$

The two-term controller (2.6) is then redesigned using $\theta=20$ for performance index $J_{A \omega}$ (2.58), while holding all other settings to be the same. In this case, the new optimal control gains obtained are

$$
\text { Case } \theta=20: \quad K_{P}=1.1653, \quad K_{I}=7.7464 \times 10^{-2} \text {. }
$$

Simulation of various disturbance inputs is given in Figures 5.2-1 to 5.2-9 for the resulting closed-loop system. A summary of the optimization results is given in Tables 5.1 and 5.2, and the detailed optimization output can be found in Appendix C.1.

Remark 5.1. It is seen that the controller designed with $\theta=20$ produces significantly less transient oscillations as compared to the case when $\theta=0$, but is slower (as expected due to the structure of the performance index when $\theta \neq 0$ ). It is to be noted that we must be able to excite the disturbances in order to carry out this optimization, and the order of the resulting controller obtained is low ( $4^{\text {th }}$ order).

\begin{tabular}{ccccccc}
\hline $\begin{array}{c}\text { Initial } \\
K_{P}\end{array}$ & $\begin{array}{c}\text { Initial } \\
K_{I}\end{array}$ & $\begin{array}{c}\text { Final } \\
K_{P}\end{array}$ & $\begin{array}{c}\text { Final } \\
K_{I}\end{array}$ & $\begin{array}{c}\text { Initial } \\
\text { cost } J\end{array}$ & $\begin{array}{c}\text { Final } \\
\text { cost } J\end{array}$ & $\begin{array}{c}\text { Number of } \\
\text { function } \\
\text { evaluations }\end{array}$ \\
\hline $\begin{array}{c}5.00 \times \\
10^{-4}\end{array}$ & $\begin{array}{c}1.00 \times \\
10^{-5}\end{array}$ & $\begin{array}{c}8.76 \times \\
10^{-2}\end{array}$ & $\begin{array}{c}6.19 \times \\
10^{-1}\end{array}$ & $\begin{array}{c}6.58 \times \\
10^{-6}\end{array}$ & $\begin{array}{c}1.97 \times \\
10^{-7}\end{array}$ & 1683 \\
\hline
\end{tabular}

Table 5.1: Summary of controller parameter optimization for the Williams and Otto chemical plant with $\theta=0$ and $\mu=10^{-6}$.

\begin{tabular}{ccccccc}
\hline $\begin{array}{c}\text { Initial } \\
K_{P}\end{array}$ & $\begin{array}{c}\text { Initial } \\
K_{I}\end{array}$ & $\begin{array}{c}\text { Final } \\
K_{P}\end{array}$ & $\begin{array}{c}\text { Final } \\
K_{I}\end{array}$ & $\begin{array}{c}\text { Initial } \\
\text { cost } J\end{array}$ & $\begin{array}{c}\text { Final } \\
\text { cost } J\end{array}$ & $\begin{array}{c}\text { Number of } \\
\text { function } \\
\text { evaluations }\end{array}$ \\
\hline $\begin{array}{c}5.00 \times \\
10^{-4}\end{array}$ & $\begin{array}{c}1.00 \times \\
10^{-5}\end{array}$ & 1.17 & $\begin{array}{c}7.75 \times \\
10^{-2}\end{array}$ & $\begin{array}{c}8.28 \times \\
10^{-4}\end{array}$ & $\begin{array}{c}6.16 \times \\
10^{-4}\end{array}$ & 1800 \\
\hline
\end{tabular}

Table 5.2: Summary of controller parameter optimization for the Williams and Otto chemical plant with $\theta=20$ and $\mu=10^{-6}$. 

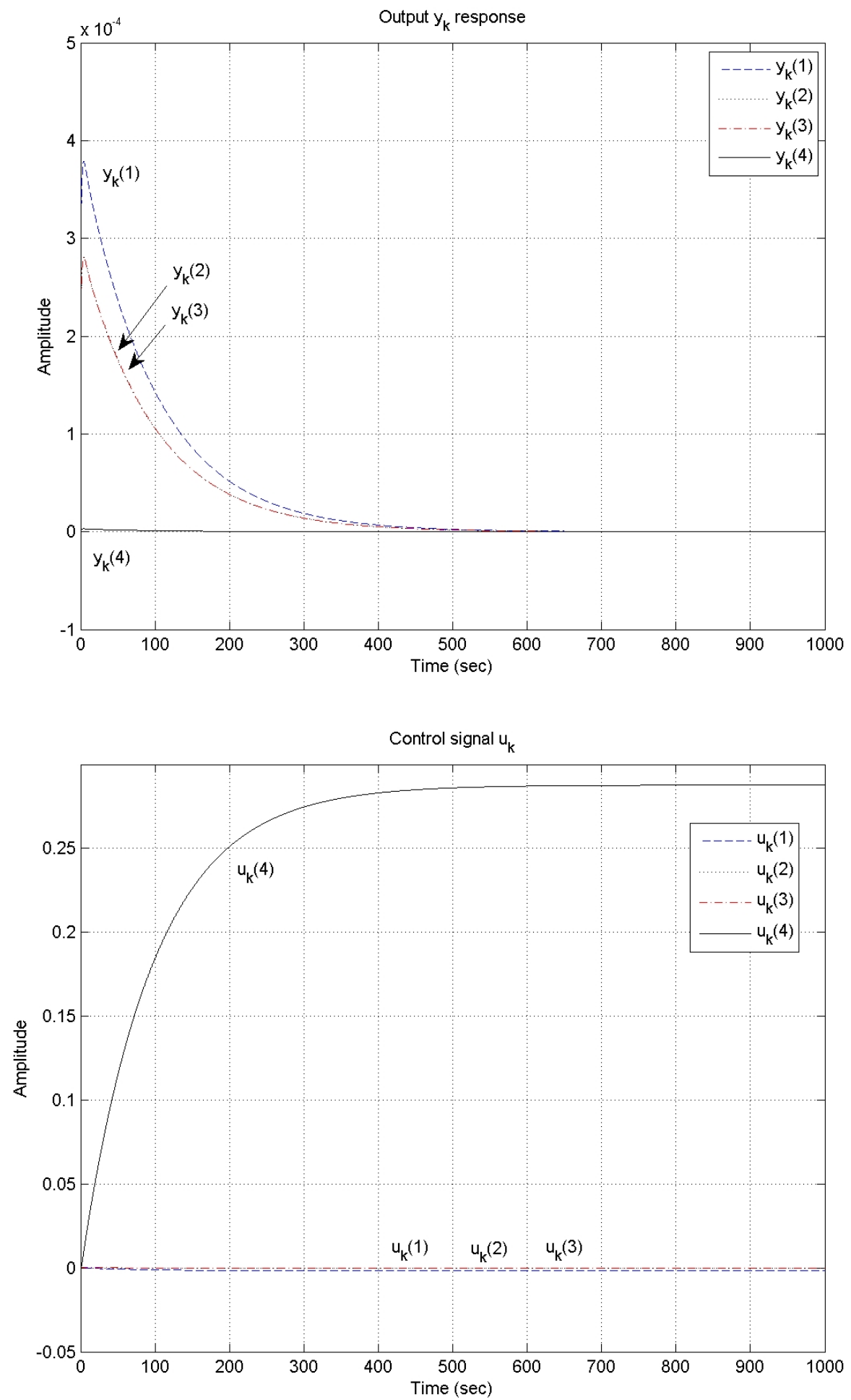

Figure 5.2-1: Williams and Otto Chemical Plant Example, Closed-Loop Response Tuning Regulator (2.17) with $\epsilon=10^{-2}$ Input $\omega=\left[\begin{array}{lll}1 & 0 & 0\end{array}\right]^{\top}$. 

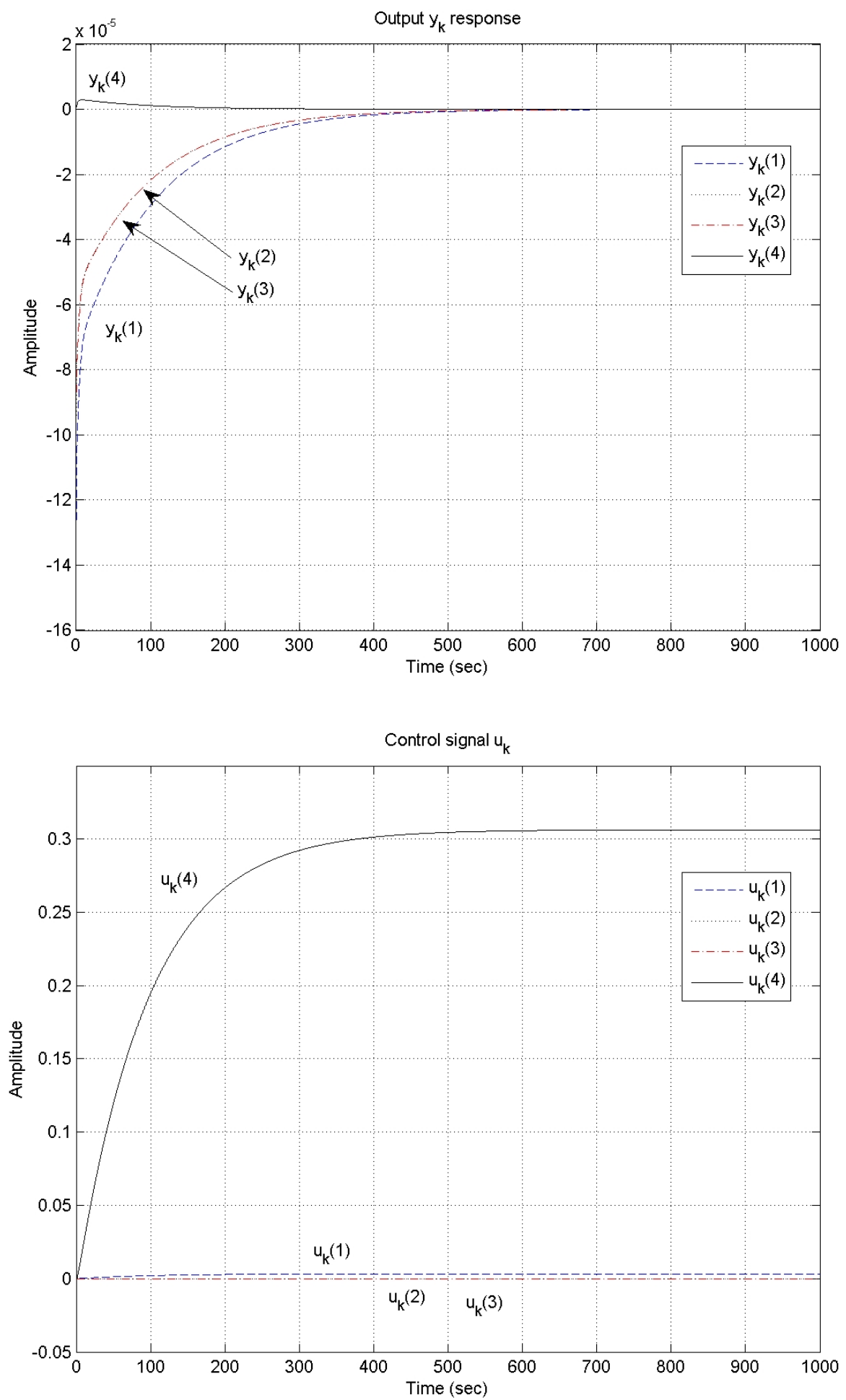

Figure 5.2-2: Williams and Otto Chemical Plant Example, Closed-Loop Response Tuning Regulator (2.17) with $\epsilon=10^{-2}$

Input $\omega=\left[\begin{array}{lll}0 & 1 & 0\end{array}\right]^{\top}$. 

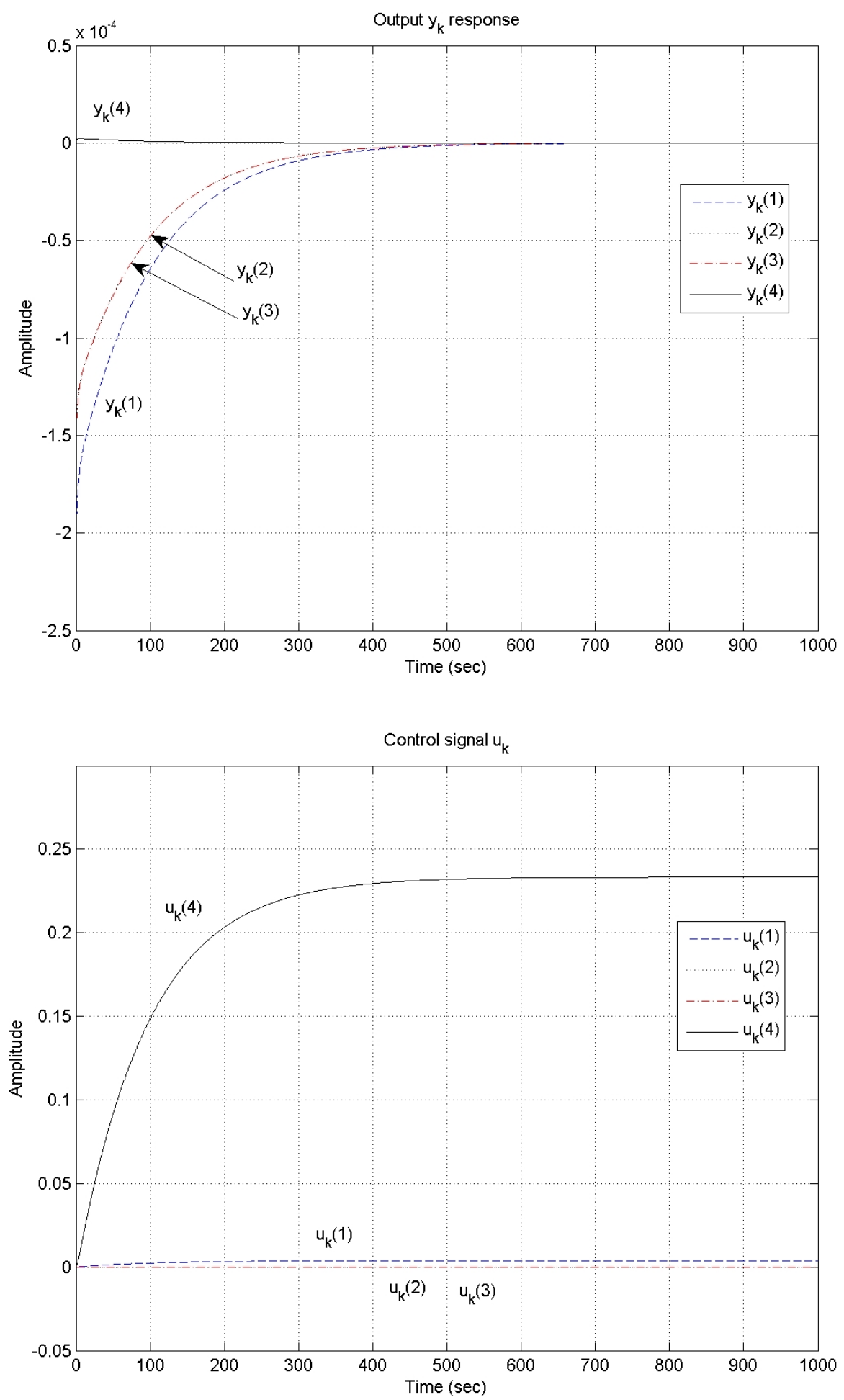

Figure 5.2-3: Williams and Otto Chemical Plant Example, Closed-Loop Response Tuning Regulator (2.17) with $\epsilon=10^{-2}$

Input $\omega=\left[\begin{array}{lll}0 & 0 & 1\end{array}\right]^{\top}$. 

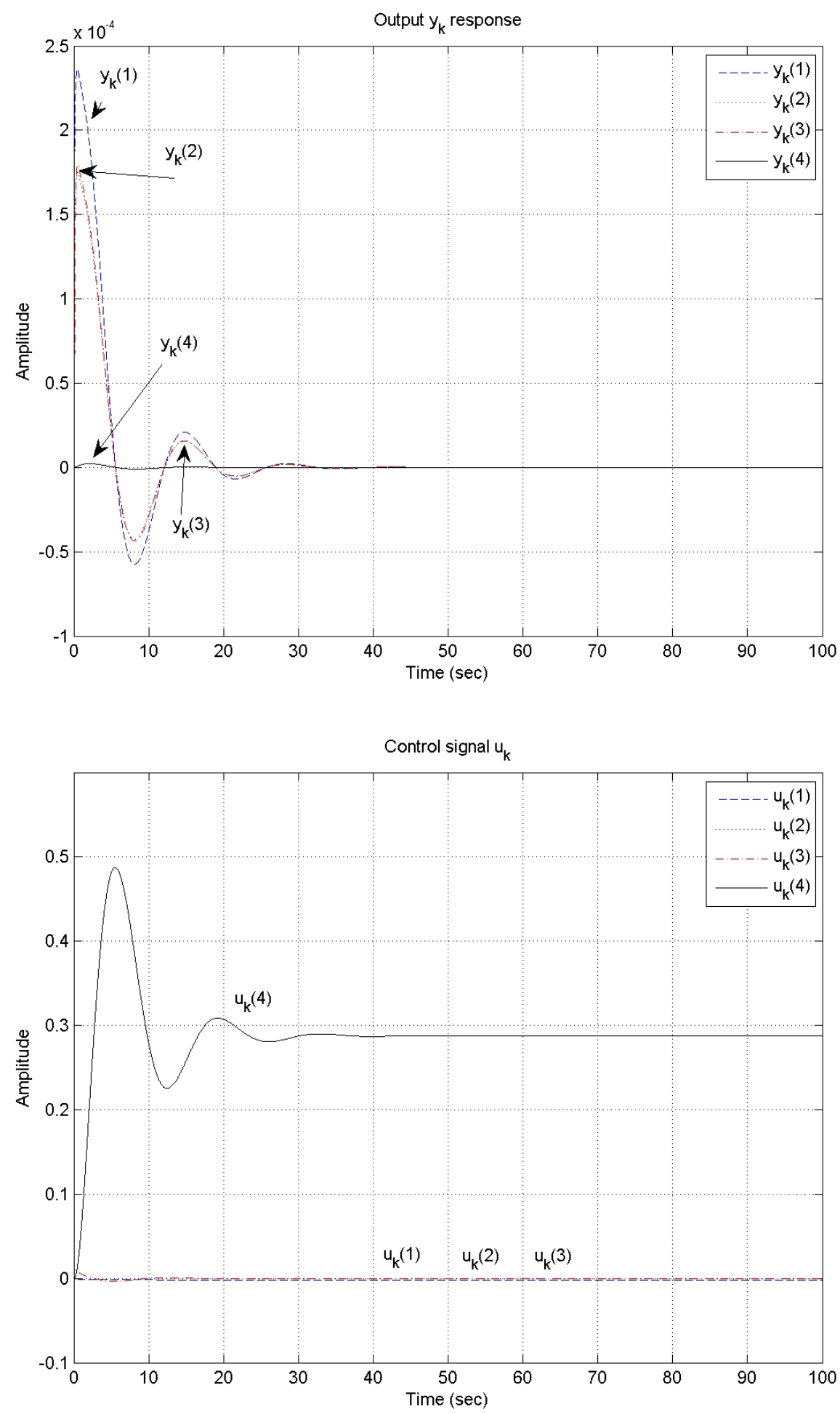

Figure 5.2-4: Williams and Otto Chemical Plant Example, Closed-Loop Response Two-Term Controller (2.6), $\theta=0$ with Controller Gains (5.1) Input $\omega=\left[\begin{array}{lll}1 & 0 & 0\end{array}\right]^{\top}$. 

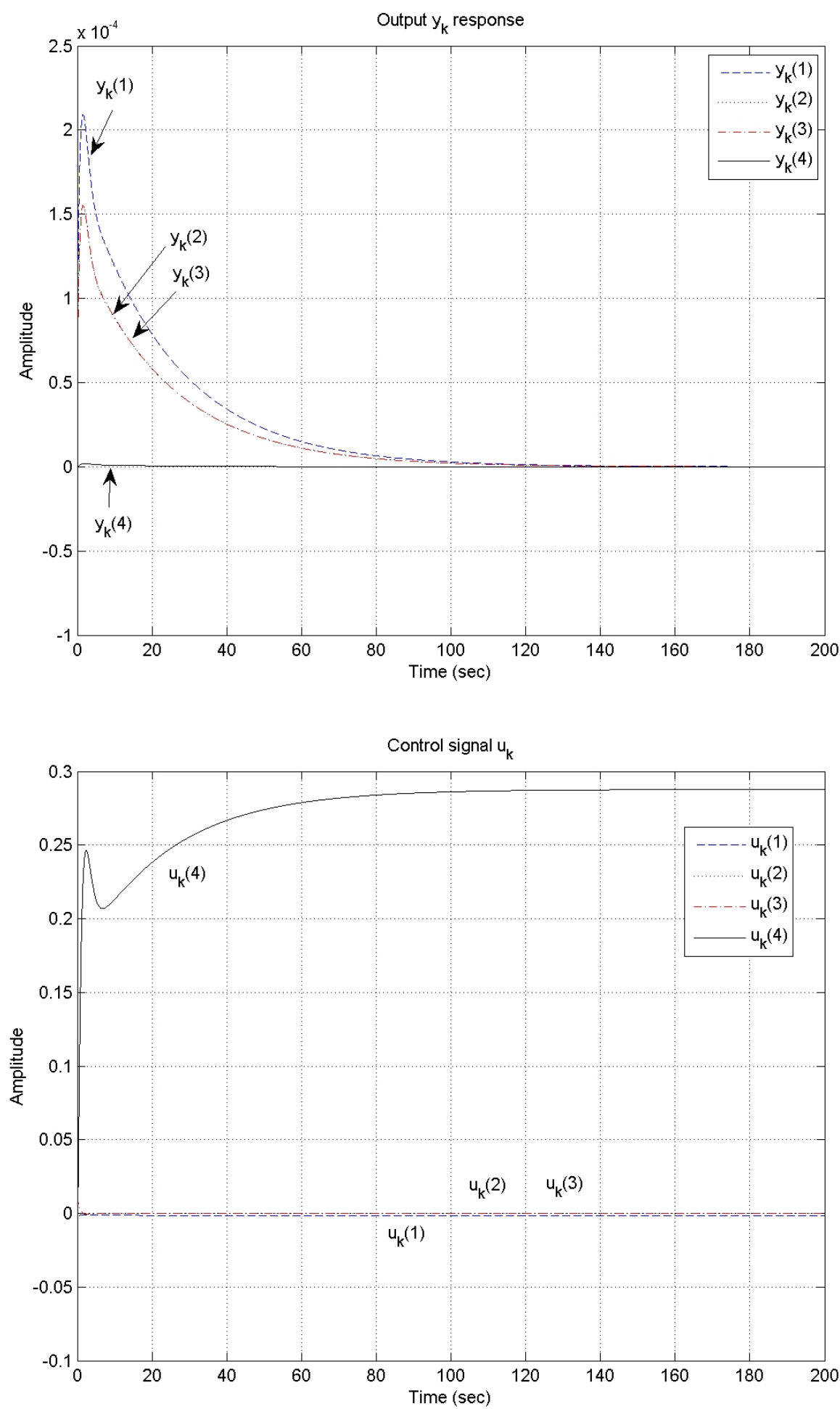

Figure 5.2-5: Williams and Otto Chemical Plant Example, Closed-Loop Response Two-Term Controller (2.6), $\theta=20$ with Controller Gains (5.2) Input $\omega=\left[\begin{array}{lll}1 & 0 & 0\end{array}\right]^{\top}$. 

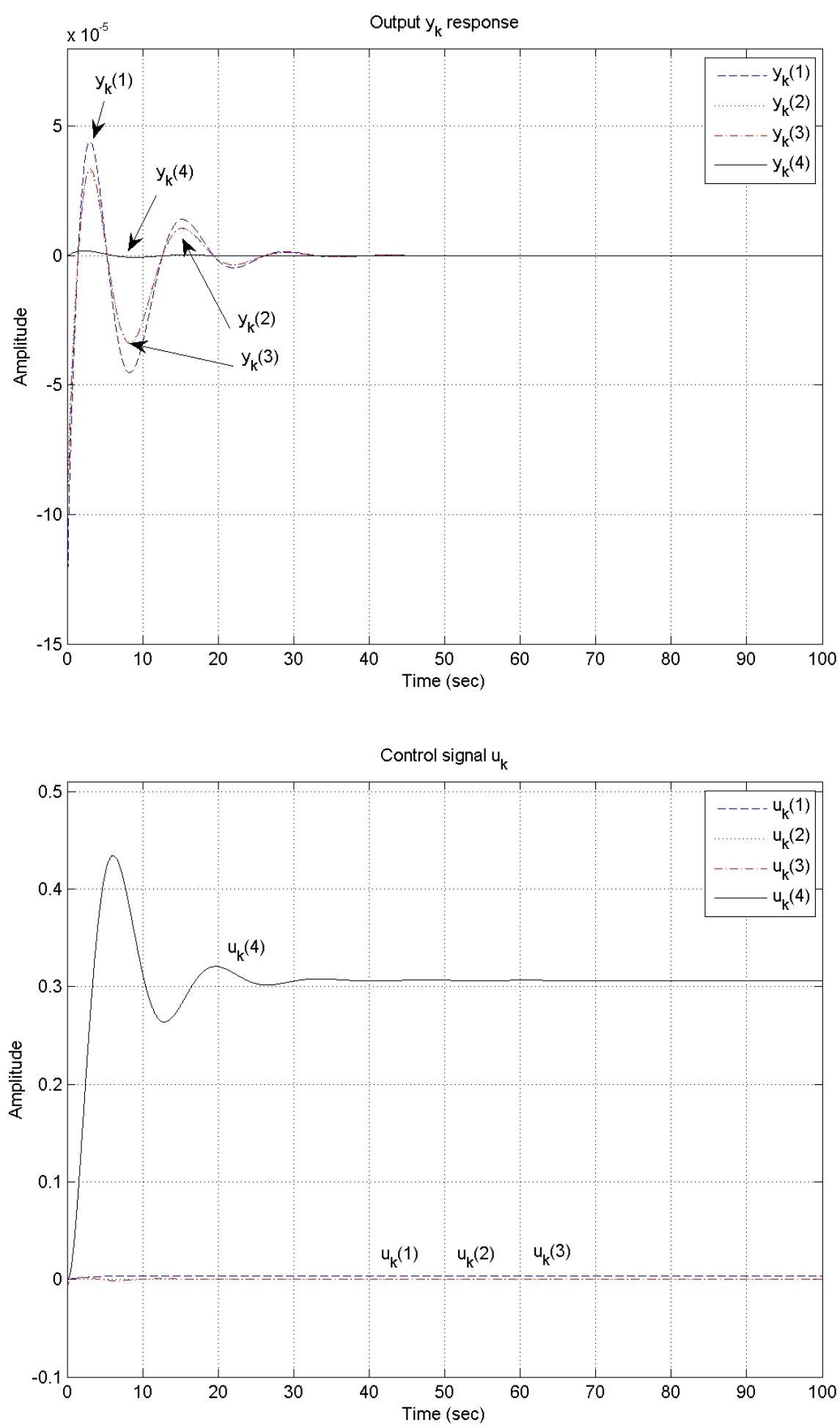

Figure 5.2-6: Williams and Otto Chemical Plant Example, Closed-Loop Response Two-Term Controller (2.6), $\theta=0$ with Controller Gains (5.1) Input $\omega=\left[\begin{array}{lll}0 & 1 & 0\end{array}\right]^{\top}$. 

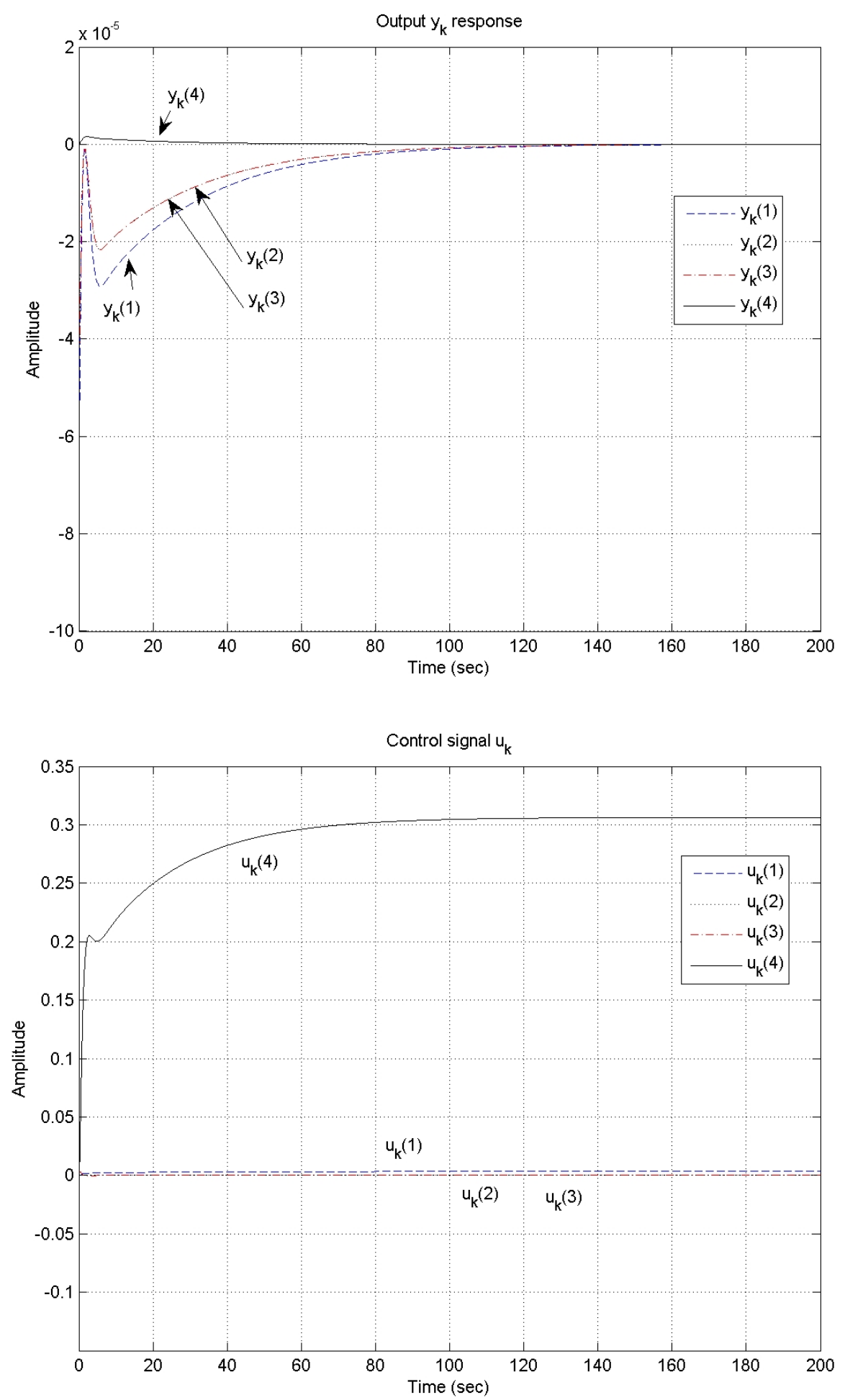

Figure 5.2-7: Williams and Otto Chemical Plant Example, Closed-Loop Response Two-Term Controller (2.6), $\theta=20$ with Controller Gains (5.2) Input $\omega=\left[\begin{array}{lll}0 & 1 & 0\end{array}\right]^{\top}$. 

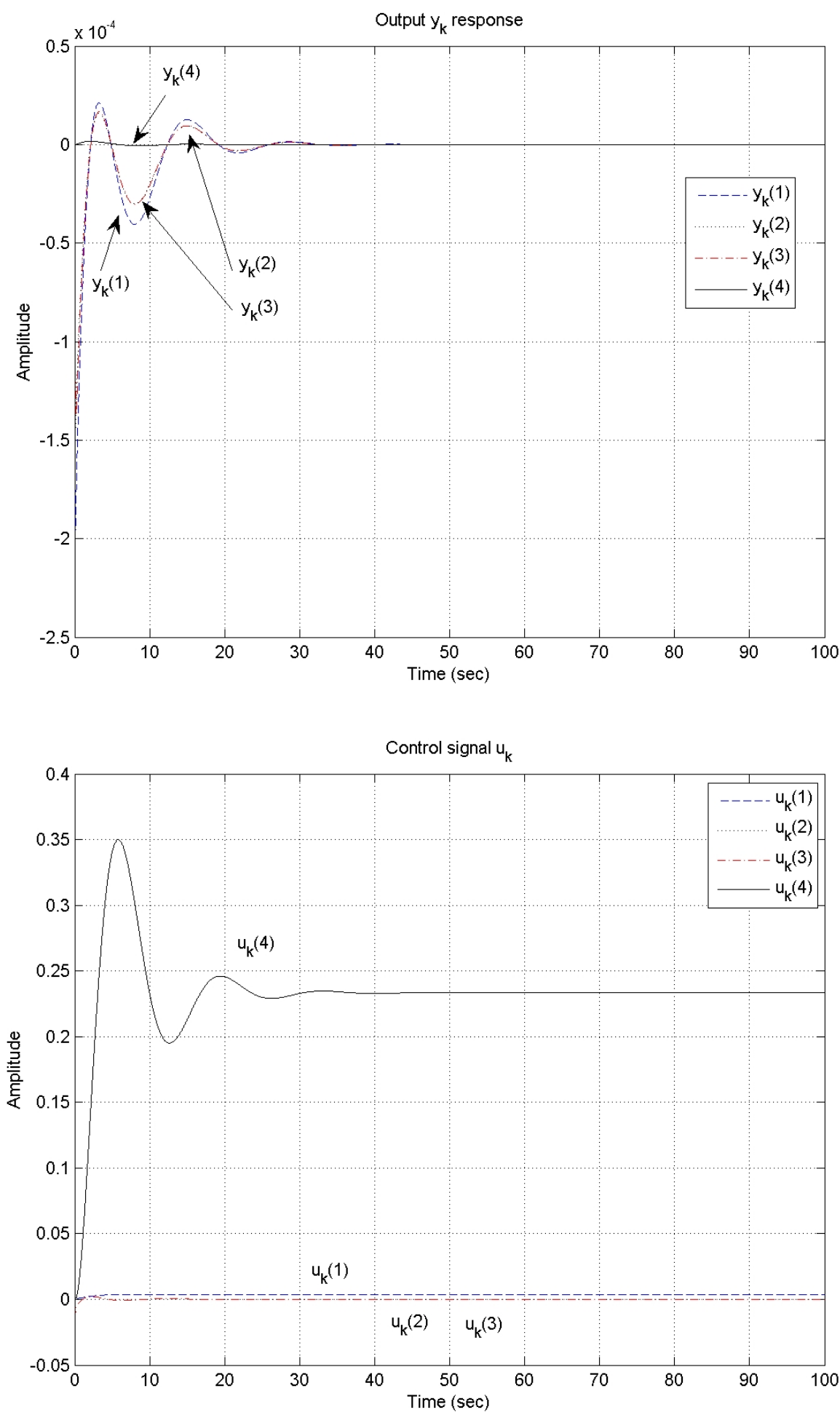

Figure 5.2-8: Williams and Otto Chemical Plant Example, Closed-Loop Response Two-Term Controller (2.6), $\theta=0$ with Controller Gains (5.1) Input $\omega=\left[\begin{array}{lll}0 & 0 & 1\end{array}\right]^{\top}$. 

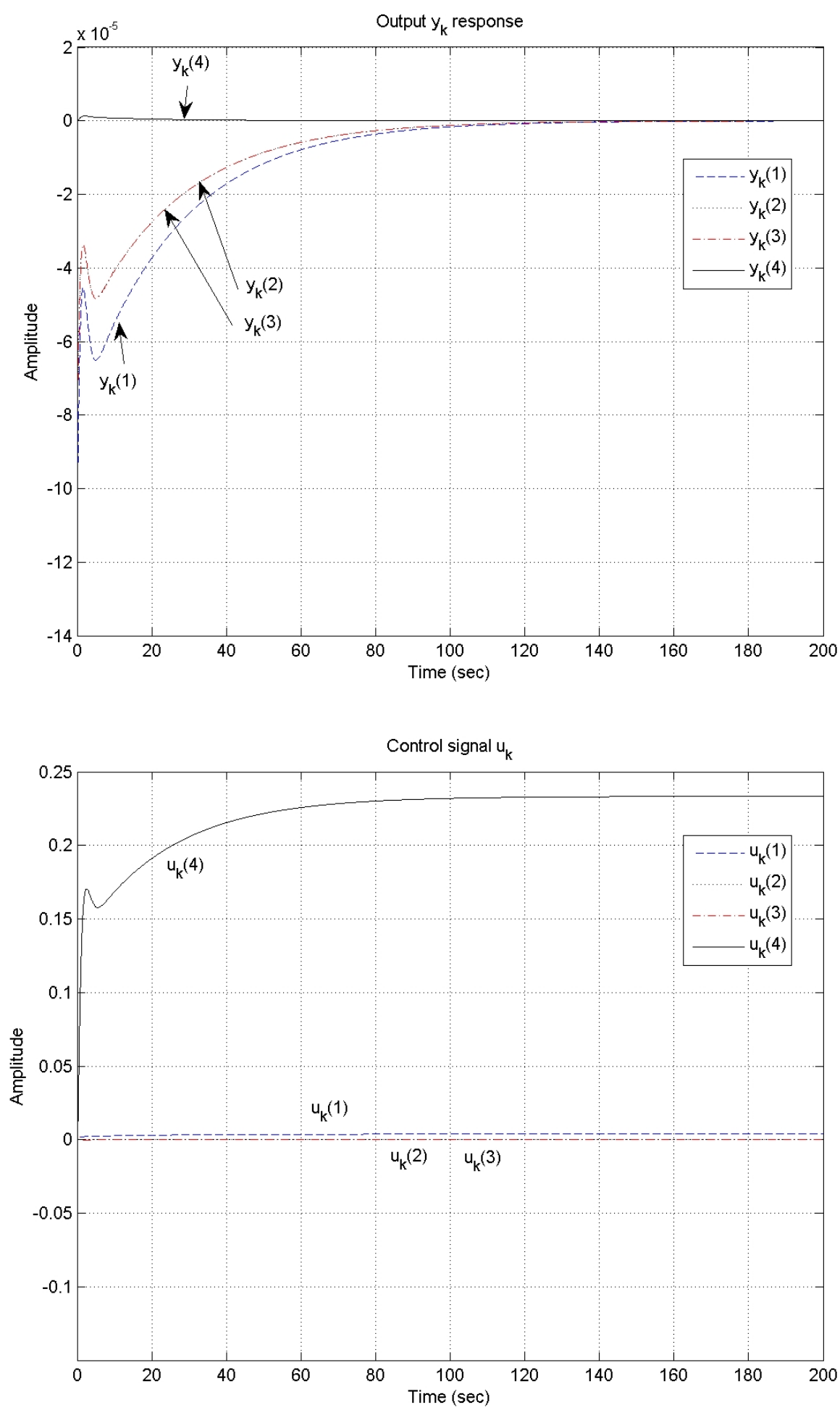

Figure 5.2-9: Williams and Otto Chemical Plant Example, Closed-Loop Response Two-Term Controller (2.6), $\theta=20$ with Controller Gains (5.2) Input $\omega=\left[\begin{array}{lll}0 & 0 & 1\end{array}\right]^{\top}$. 


\subsection{Summary}

The proposed controller design approach is applied to a large-scale system that is openloop stable and minimum phase. The purpose is to experimentally find a controller that minimizes the average cost of performance index $J_{A}(2.47)$ over all disturbance signals uniformly distributed on a unit ball. The resulting controller obtained is $4^{\text {th }}$ order for this $41^{\text {st }}$ order system and the closed-loop output response is fast. Setting $\theta \neq 0$ in the performance index $J_{A}(2.47)$ produces less transient oscillations as compared to the case when $\theta=0$. 


\section{Chapter 6}

\section{Turbo-Generator Example (Nonminimum Phase System)}

\subsection{Description of the Turbo-Generator}

The Koeberg turbo-generator in [41] is a $6^{\text {th }}$ order system with 2 inputs and 2 outputs. The system is described by the following continuous linear time-invariant model:

$$
\begin{aligned}
\dot{x}(t) & =\mathcal{A} x(t)+\mathcal{B} u(t) \\
y(t) & =\mathcal{C} x(t)+\mathcal{D} u(t)
\end{aligned}
$$

where $\mathcal{A}, \mathcal{B}, \mathcal{C}$, and $\mathcal{D}$ are given by

$$
\mathcal{A}=\left[\begin{array}{cccccc}
-18.446 & 4.2263 & -2.2830 & 0.22600 & 0.42200 & -0.0951 \\
-4.0977 & -6.0706 & 5.6825 & -0.69660 & -1.2246 & 0.2873 \\
1.4449 & 1.4336 & -2.6477 & 0.60920 & 0.8979 & -0.2300 \\
-0.0093 & 0.23020 & -0.5002 & -0.17640 & -6.3152 & 0.1350 \\
-0.0464 & -0.3489 & 0.7238 & 6.3117 & -0.6886 & 0.3645 \\
-0.0602 & -0.23610 & 0.2300 & 0.0915 & -0.3214 & -0.2087
\end{array}\right]
$$




$$
\begin{aligned}
& \mathcal{B}=\left[\begin{array}{cc}
-0.2748 & 3.1463 \\
-0.0501 & -9.3737 \\
-0.1550 & 7.4296 \\
0.0716 & -4.9176 \\
-0.0814 & -10.265 \\
0.0244 & 13.794
\end{array}\right] \\
& \mathcal{C}=\left[\begin{array}{cccccc}
0.5971 & -0.7697 & 4.8850 & 4.8608 & -9.8177 & -8.8610 \\
3.1013 & 9.3422 & -5.6000 & -0.7490 & 2.9974 & 10.572
\end{array}\right] \\
& \mathcal{D}=\left[\begin{array}{ll}
0 & 0 \\
0 & 0
\end{array}\right] \text {. }
\end{aligned}
$$

The eigenvalues of this continuous system are

$$
\{-15.873,-10.387,-0.34925 \pm 6.3444 i,-1.0444,-0.23455\}
$$

and the transmission zeros are given by

$$
\{2170.6,22.623,-33.628 \pm 7.2463 i\} .
$$

If we sample the system with $h=0.1$, the resultant sampled system is open-loop stable and nonminimum phase with eigenvalues at

$$
\{0.20448,0.35391,0.77776 \pm 0.57238 i, 0.90083,0.97682\}
$$

and with transmission zeros given by

$$
\{20.076,0.042514,-0.15968,-1.0262\} \text {. }
$$

It is to be noted that the mathematical model of the plant (6.1) is used in this chapter only to carry out simulations, but it is not used for controller design.

Since actuator constraints were not given in [41], we assume that the operating range of the control signals is given by

$$
\begin{aligned}
& -5 \leq u_{k}(1) \leq 5 \\
& -5 \leq u_{k}(2) \leq 5 .
\end{aligned}
$$

Open-loop responses of this system are given in Figure 6.1. The steady-state tracking matrix $\Gamma(2.2)$ is obtained experimentally to be:

$$
\Gamma=\left[\begin{array}{cc}
-1.46 & -585 \\
0.557 & 664
\end{array}\right] \text {. }
$$



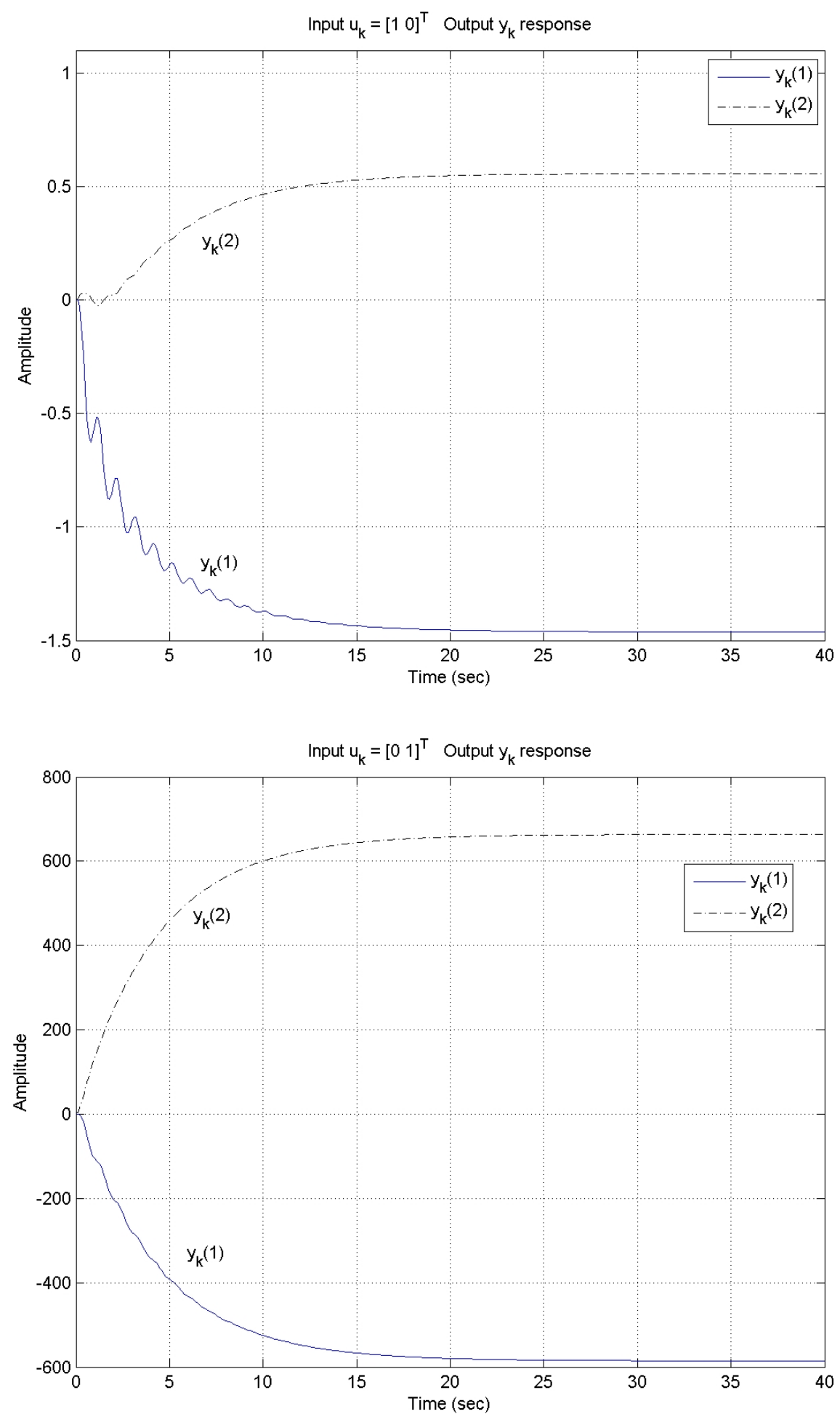

Figure 6.1: Turbo-Generator Example, Open-Loop Response. 


\subsection{Control Design 1 for Turbo-Generator}

In this study, it is desired to design a controller for the case of tracking constant set points. The tuning regulator (2.17) with $\epsilon=10^{-3}$ is first applied to the plant and the two-term controller (2.6) is then obtained by minimizing the performance index $J_{A y_{\text {ref }}}$ (2.59) using $\theta=0, \mu=10^{-4}$. The optimization is carried out under the system settings of:

$$
x_{0}=\left[\begin{array}{l}
0 \\
0
\end{array}\right], \eta_{0}=\left[\begin{array}{l}
0 \\
0
\end{array}\right], \text { and } \mathcal{Z}_{y_{r e f}}=\left\{\left[\begin{array}{l}
0 \\
1
\end{array}\right],\left[\begin{array}{c}
\frac{1}{\sqrt{2}} \\
\frac{1}{\sqrt{2}}
\end{array}\right],\left[\begin{array}{l}
1 \\
0
\end{array}\right],\left[\begin{array}{c}
\frac{1}{\sqrt{2}} \\
-\frac{1}{\sqrt{2}}
\end{array}\right]\right\} ;
$$

in this case the optimal control gains $K_{P}$ and $K_{I}$ are found to be

$$
\text { Case } \theta=0: \quad K_{P}=7.1299 \times 10^{-1}, \quad K_{I}=2.0062 .
$$

The two-term controller (2.6) is then redesigned using $\theta=10$ for performance index $J_{A y_{\text {ref }}}(2.59)$, while holding all other settings to be the same. In this case, the new optimal control gains are

$\underline{\text { Better Controller }}$ Case $\theta=10: \quad K_{P}=4.5141 \times 10^{-1}, K_{I}=1.3764 \times 10^{-1}$.

Simulation of the tracking reference input is given in Figures 6.2-1 to 6.2-6 for the resulting closed-loop system. A summary of the optimization results is given in Tables 6.1 and 6.2, and the detailed optimization output can be found in Appendix C.2.

Remark 6.1. When the Nelder-Mead algorithm converged for the first time, the controller gains $K_{P}=6.6778 \times 10^{-1}$, and $K_{I}=2.1259 \times 10^{-2}$ were obtained and the cost was $J=38.3182$ (see Appendix C.2). With this set of controller gains, the closed-loop system is actually unstable with the output $y_{k}$ slowly growing unbounded. The cost, however, was not assigned a value of $10^{20}$ since the control input $u_{k}$ never reached the actuator saturation bounds before the experiment was terminated after $T=10000$ seconds. This implies that a longer experimental duration will be required for the cost value to better reflect system instability. Nevertheless, restarting the Nelder-Mead algorithm from this unstable point in the controller parameter space gave rise to a set of optimal gains $K_{P}=7.1299 \times 10^{-1}$ and $K_{I}=2.0062$ that ensured closed-loop stability.

Remark 6.2. It is seen that the controller found with $\theta=10$ gives a more desirable transient response compared to the case when $\theta=0$, which is highly oscillatory. The oscillation arises from the physics of the system where the turbo-generator has a resonance 
frequency at $6.35 \mathrm{rad} / \mathrm{sec}$ with a damping factor of only 0.05 [41]. The controller design approach is shown to be effective for this open-loop stable, non-minimum phase system.

\begin{tabular}{ccccccc}
\hline Initial & Initial & Final & Final & Initial & Final & $\begin{array}{c}\text { Number of } \\
\text { function } \\
K_{P}\end{array}$ \\
$K_{I}$ & $K_{P}$ & $K_{I}$ & cost $J$ & cost $J$ & $\begin{array}{c}\text { cvaluations } \\
\text { evalu }\end{array}$ \\
\hline $5.00 \times$ & $1.00 \times$ & $7.13 \times$ & 2.01 & 496.69 & 1.59 & 1108 \\
$10^{-4}$ & $10^{-5}$ & $10^{-1}$ & & & & \\
\hline
\end{tabular}

Table 6.1: Summary of controller parameter optimization $A$ for the Turbo-Generator with $\theta=0$ and $\mu=10^{-4}$.

\begin{tabular}{ccccccc}
\hline Initial & Initial & Final & Final & Initial & Final & $\begin{array}{c}\text { Number of } \\
\text { function } \\
K_{P}\end{array}$ \\
$K_{I}$ & $K_{P}$ & $K_{I}$ & $\operatorname{cost} J$ & cost $J$ & $\begin{array}{c}\text { cvaluations } \\
\text { evalu }\end{array}$ \\
\hline $5.00 \times$ & $1.00 \times$ & $4.51 \times$ & $1.38 \times$ & & & 876 \\
$10^{-4}$ & $10^{-5}$ & $10^{-1}$ & $10^{-1}$ & & & \\
\hline
\end{tabular}

Table 6.2: Summary of controller parameter optimization $A$ for the Turbo-Generator with $\theta=10$ and $\mu=10^{-4}$. 

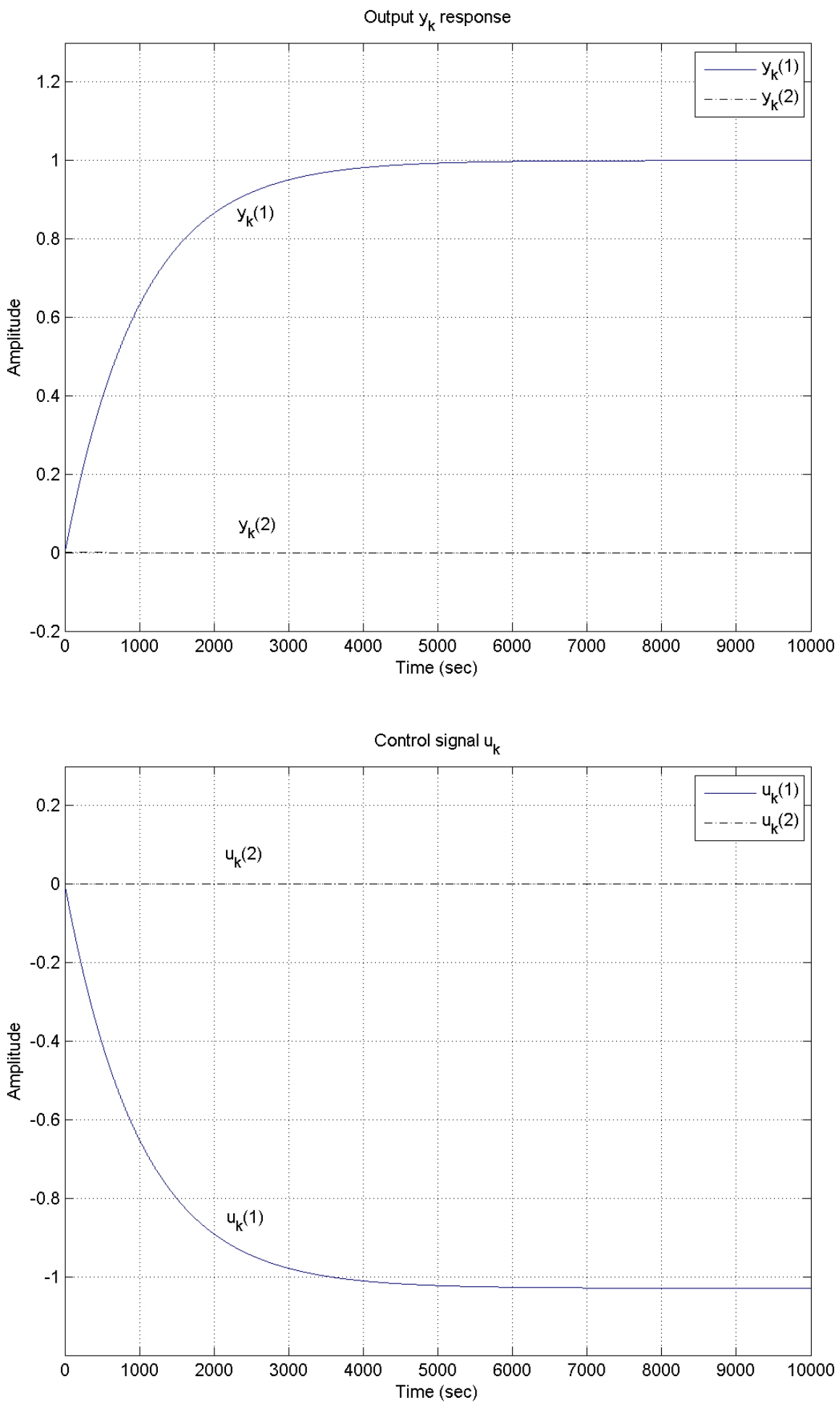

Figure 6.2-1: Turbo-Generator Example, Closed-Loop Response Tuning Regulator (2.17) with $\epsilon=10^{-3}$

$$
\text { Input } y_{\text {ref }}=\left[\begin{array}{ll}
1 & 0
\end{array}\right]^{\top}
$$

Design 1. 

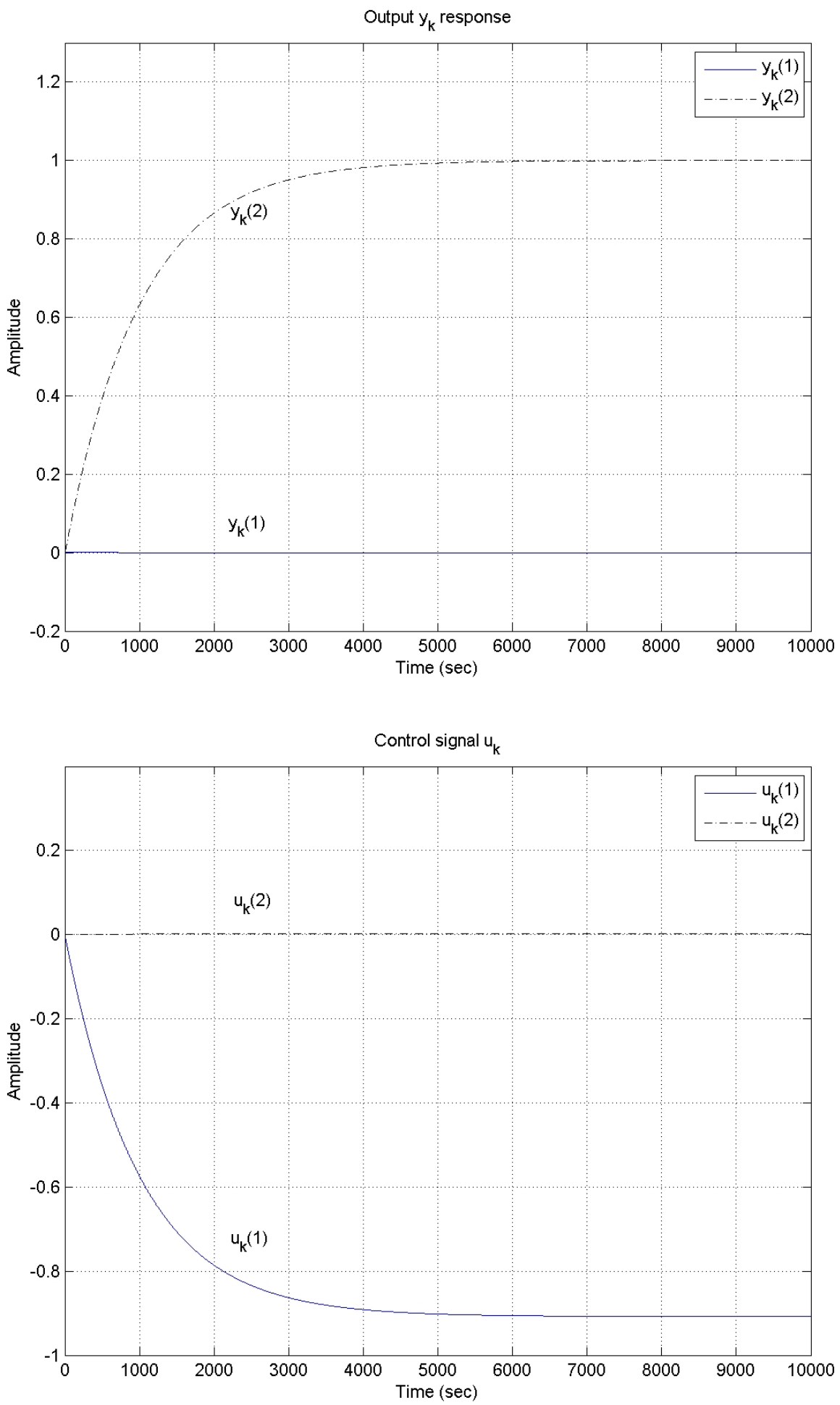

Figure 6.2-2: Turbo-Generator Example, Closed-Loop Response Tuning Regulator (2.17) with $\epsilon=10^{-3}$

$$
\text { Input } y_{\text {ref }}=\left[\begin{array}{ll}
0 & 1
\end{array}\right]^{\top}
$$

Design 1. 

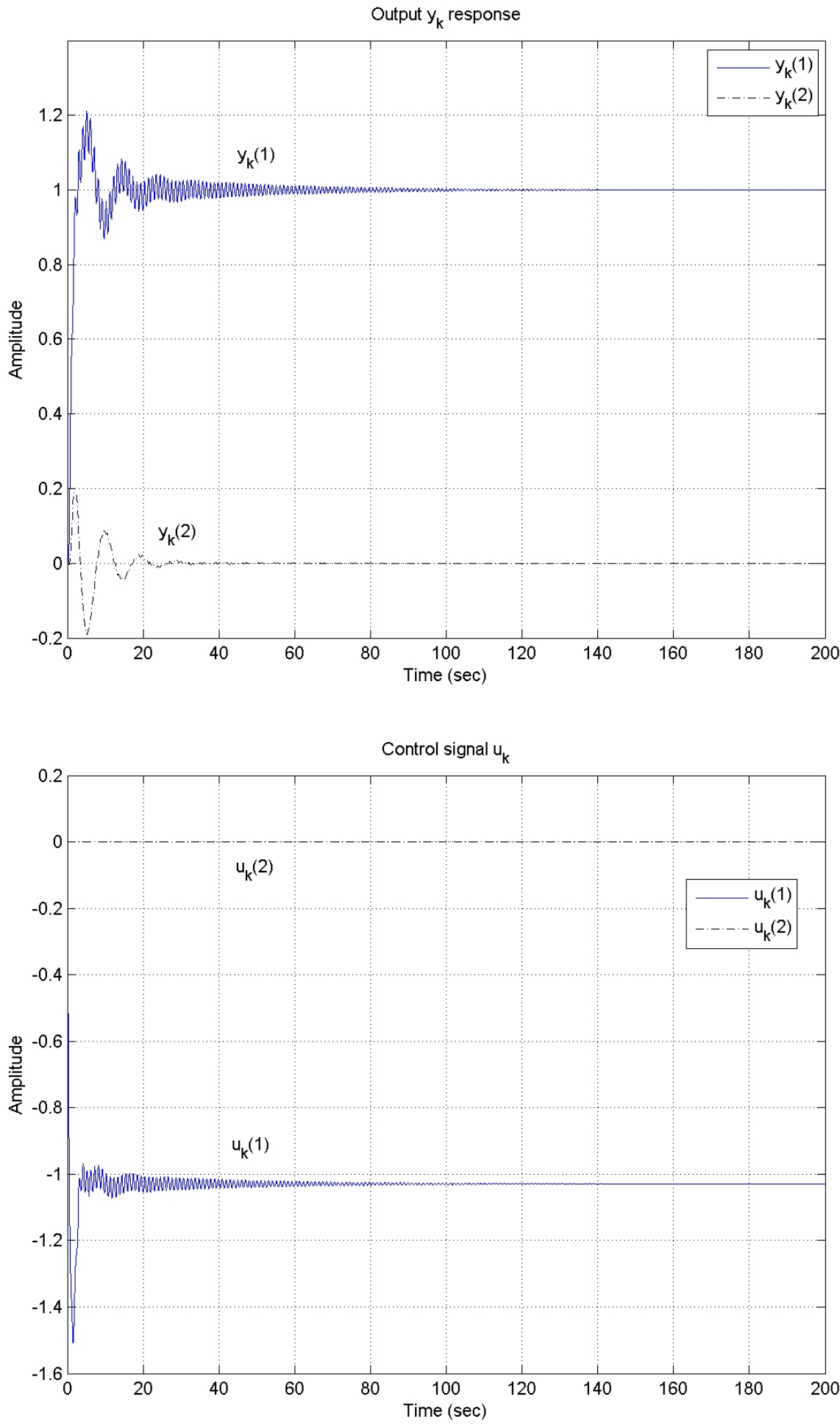

Figure 6.2-3: Turbo-Generator Example, Closed-Loop Response Two-Term Controller (2.6), $\theta=0$ with Controller Gains (6.2)

$$
\text { Input } y_{\text {ref }}=\left[\begin{array}{ll}
1 & 0
\end{array}\right]^{\top}
$$

Design 1. 

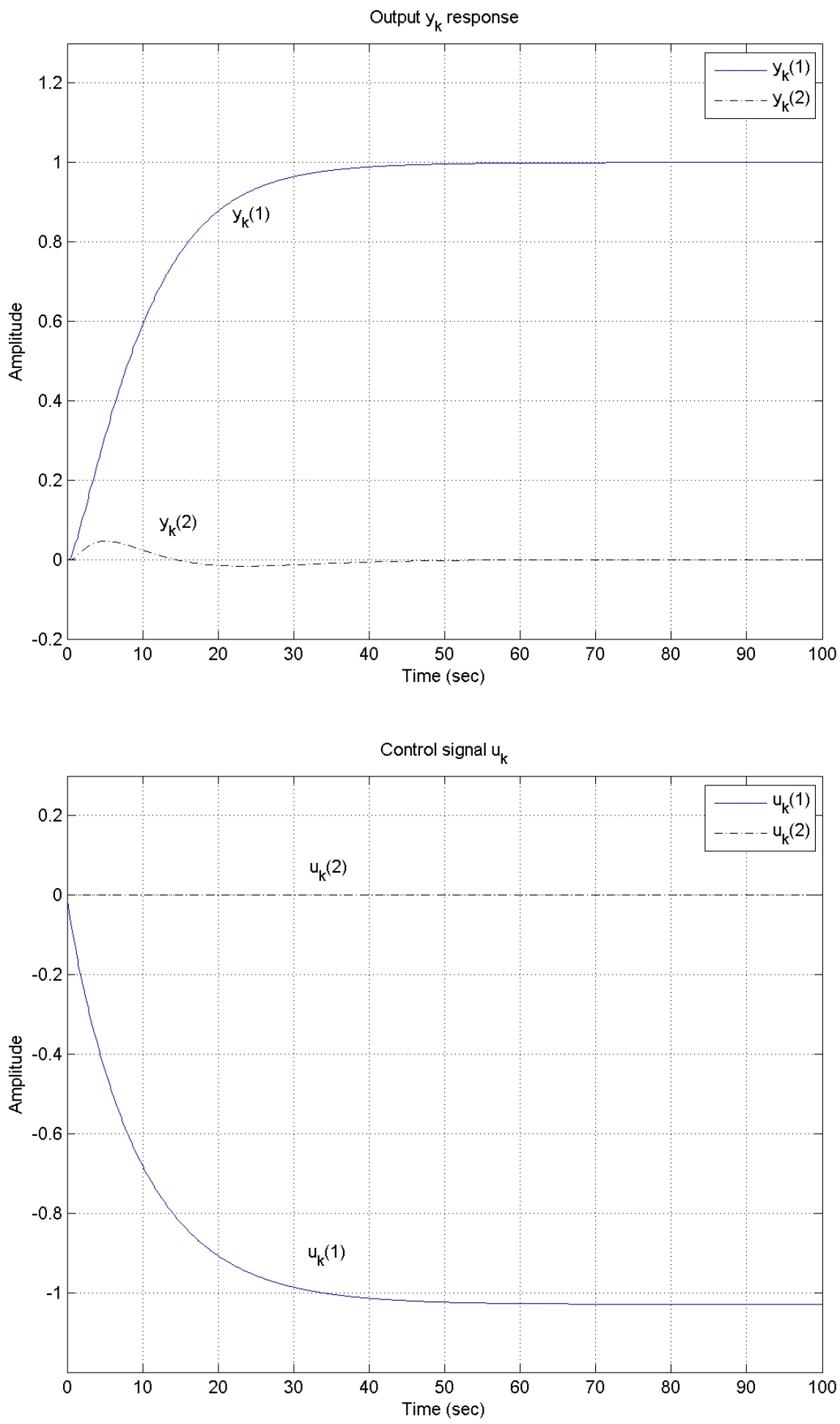

Figure 6.2-4: Turbo-Generator Example, Closed-Loop Response Two-Term Controller (2.6), $\theta=10$ with Controller Gains (6.3) (Better Controller) Input $y_{r e f}=\left[\begin{array}{ll}1 & 0\end{array}\right]^{\top}$ Design 1. 

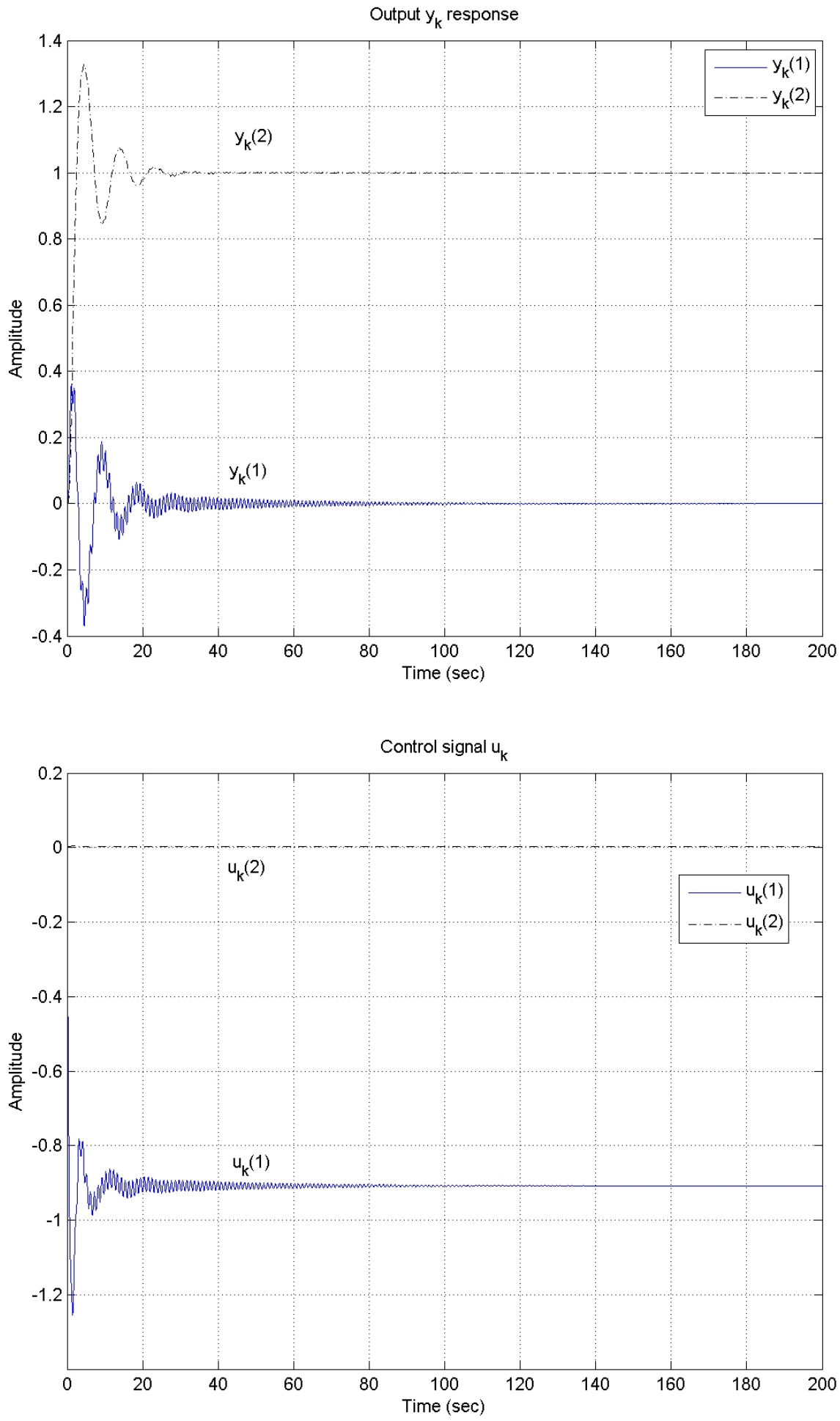

Figure 6.2-5: Turbo-Generator Example, Closed-Loop Response Two-Term Controller (2.6), $\theta=0$ with Controller Gains (6.2)

$$
\text { Input } y_{\text {ref }}=\left[\begin{array}{ll}
0 & 1
\end{array}\right]^{\top}
$$

Design 1. 

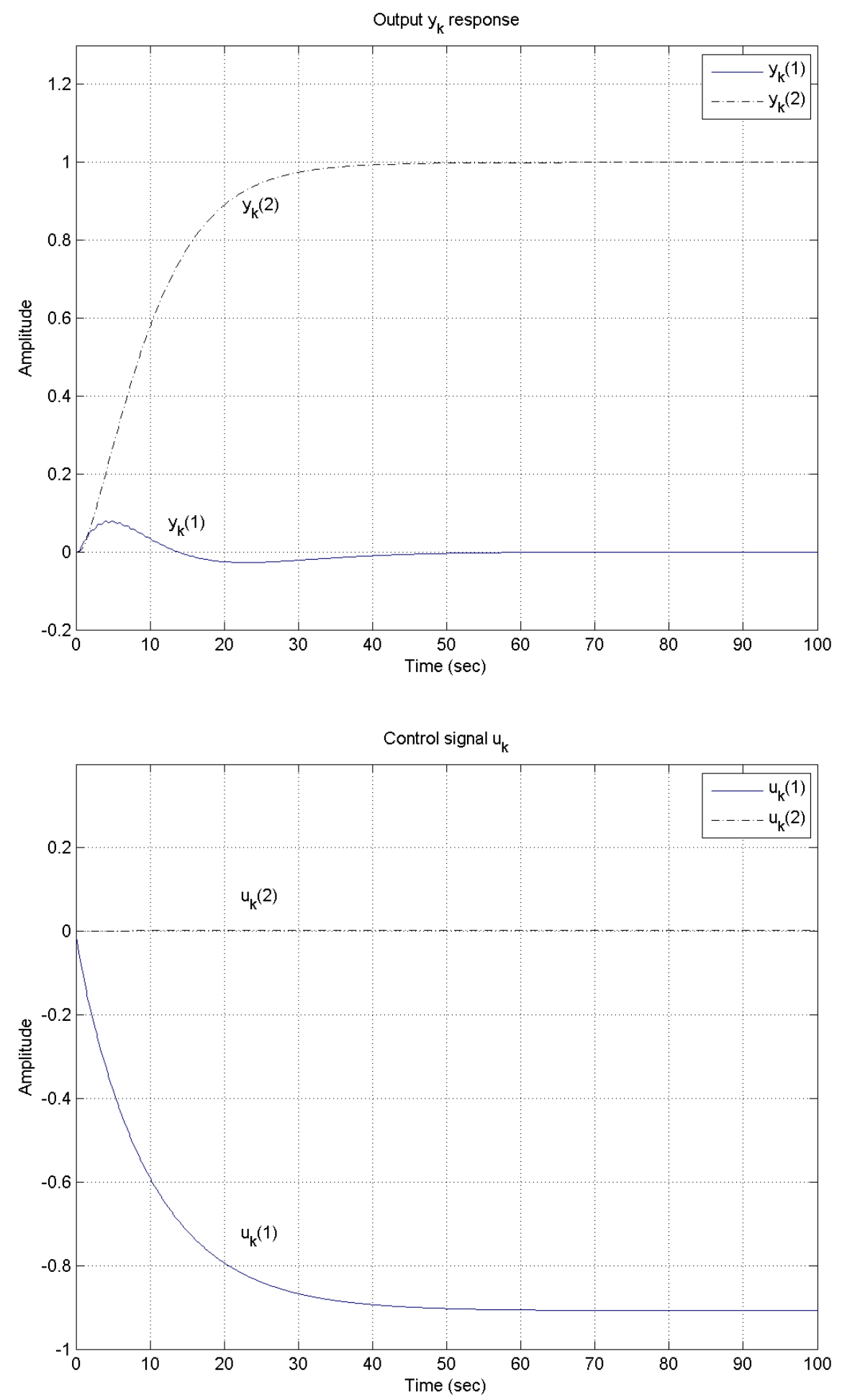

Figure 6.2-6: Turbo-Generator Example, Closed-Loop Response Two-Term Controller (2.6), $\theta=10$ with Controller Gains (6.3) (Better Controller) Input $y_{\text {ref }}=\left[\begin{array}{ll}0 & 1\end{array}\right]^{\top}$ Design 1. 


\subsection{Control Design 2 for Turbo-Generator}

The tuning regulator (2.17) with $\epsilon=10^{-3}$ is first applied to the plant and the two-term controller (2.6) is then obtained by minimizing the performance index $J_{B \eta_{0}}(2.79)$ using $\theta=0, \mu=10^{-4}$. The optimization is carried out under the system settings of:

$$
x_{0}=\left[\begin{array}{l}
0 \\
0
\end{array}\right], y_{\text {ref }}=\left[\begin{array}{l}
0 \\
0
\end{array}\right], \text { and } \mathcal{Z}_{\eta_{0}}=\left\{\left[\begin{array}{l}
0 \\
1
\end{array}\right],\left[\begin{array}{c}
\frac{1}{\sqrt{2}} \\
\frac{1}{\sqrt{2}}
\end{array}\right],\left[\begin{array}{l}
1 \\
0
\end{array}\right],\left[\begin{array}{c}
\frac{1}{\sqrt{2}} \\
-\frac{1}{\sqrt{2}}
\end{array}\right]\right\}
$$

in this case the optimal control gains $K_{P}$ and $K_{I}$ are found to be

$$
\text { Case } \theta=0: \quad K_{P}=7.1299 \times 10^{-1}, \quad K_{I}=2.0062
$$

The two-term controller (2.6) is then redesigned using $\theta=10$ for performance index $J_{B \eta_{0}}$ (2.79), while holding all other settings to be the same. In this case, the new optimal control gains are

$$
\underline{\text { Better Controller }} \text { Case } \theta=10: \quad K_{P}=4.5141 \times 10^{-1}, K_{I}=1.3764 \times 10^{-1} \text {. }
$$

Simulation of servocompensator's initial condition input is given in Figures 6.3-1 to 6.3-6 for the resulting closed-loop system. A summary of the optimization results is given in Tables 6.3 and 6.4, and the detailed optimization output can be found in Appendix C.2.

Remark 6.3. It is seen that the controllers found by minimizing $J_{B \eta_{0}}(2.79)$ are identical to those obtained by minimizing $J_{A y_{r e f}}(2.59)$. The approach used in Control Design 2 provides an alternative way of tuning which requires only an offset in the initial conditions of the servocompensator.

\begin{tabular}{ccccccc}
\hline Initial & Initial & Final & Final & Initial & Final & $\begin{array}{c}\text { Number of } \\
\text { function } \\
K_{P}\end{array}$ \\
$K_{I}$ & $K_{P}$ & $K_{I}$ & cost $J$ & cost $J$ & $\begin{array}{c}\text { cvaluations } \\
\text { evalu }\end{array}$ \\
\hline $5.00 \times$ & $1.00 \times$ & $7.13 \times$ & 2.01 & 496.69 & 1.59 & 1264 \\
$10^{-4}$ & $10^{-5}$ & $10^{-1}$ & & & & \\
\hline
\end{tabular}

Table 6.3: Summary of controller parameter optimization $B$ for the Turbo-Generator with $\theta=0$ and $\mu=10^{-4}$. 


\begin{tabular}{|c|c|c|c|c|c|c|}
\hline $\begin{array}{c}\text { Initial } \\
K_{P}\end{array}$ & $\begin{array}{c}\text { Initial } \\
K_{I}\end{array}$ & $\begin{array}{c}\text { Final } \\
K_{P}\end{array}$ & $\begin{array}{c}\text { Final } \\
K_{I}\end{array}$ & $\begin{array}{l}\text { Initial } \\
\text { cost } J\end{array}$ & $\begin{array}{l}\text { Final } \\
\text { cost } J\end{array}$ & $\begin{array}{c}\text { Number of } \\
\text { function } \\
\text { evaluations }\end{array}$ \\
\hline $\begin{array}{c}5.00 \times \\
10^{-4}\end{array}$ & $\begin{array}{c}1.00 \times \\
10^{-5}\end{array}$ & $\begin{array}{c}4.51 \times \\
10^{-1}\end{array}$ & $\begin{array}{c}1.38 \times \\
10^{-1}\end{array}$ & 486.74 & 1.20 & 876 \\
\hline
\end{tabular}

Table 6.4: Summary of controller parameter optimization $B$ for the Turbo-Generator with $\theta=10$ and $\mu=10^{-4}$. 

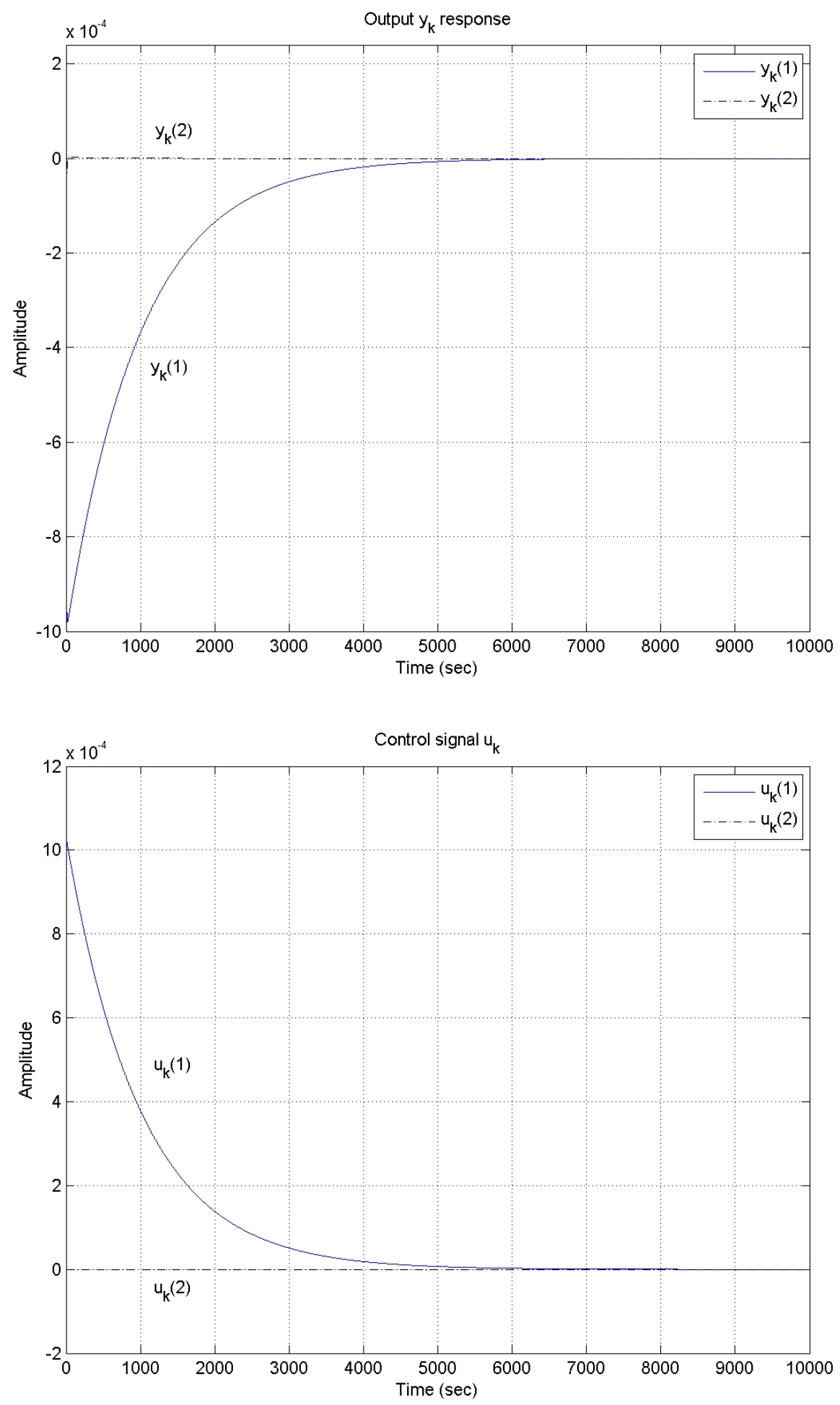

Figure 6.3-1: Turbo-Generator Example, Closed-Loop Response Tuning Regulator (2.17) with $\epsilon=10^{-3}$

$$
\text { Input } \eta_{0}=\left[\begin{array}{ll}
1 & 0
\end{array}\right]^{\top}
$$

Design 2 . 

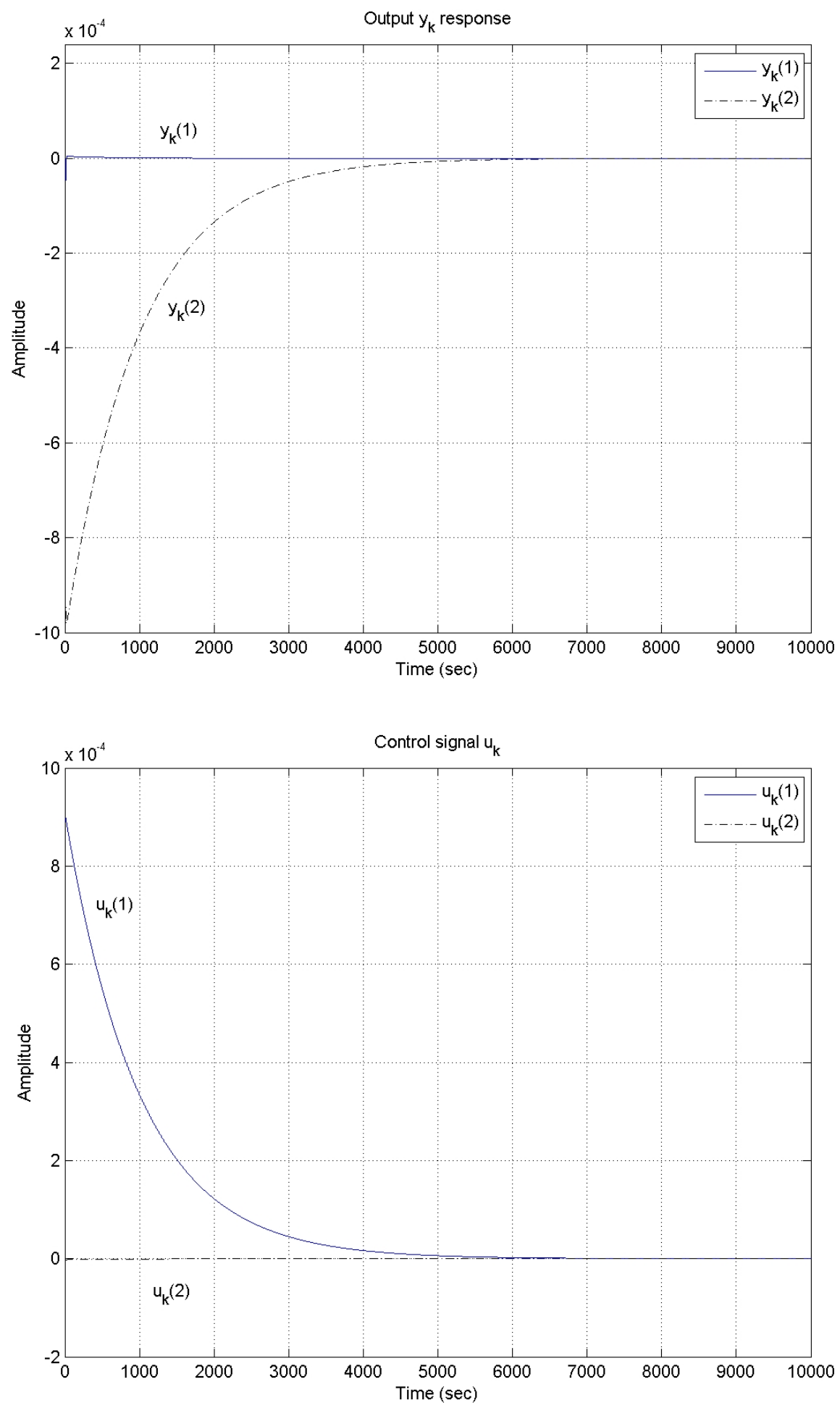

Figure 6.3-2: Turbo-Generator Example, Closed-Loop Response Tuning Regulator (2.17) with $\epsilon=10^{-3}$

$$
\text { Input } \eta_{0}=\left[\begin{array}{ll}
0 & 1
\end{array}\right]^{\top}
$$

Design 2 . 

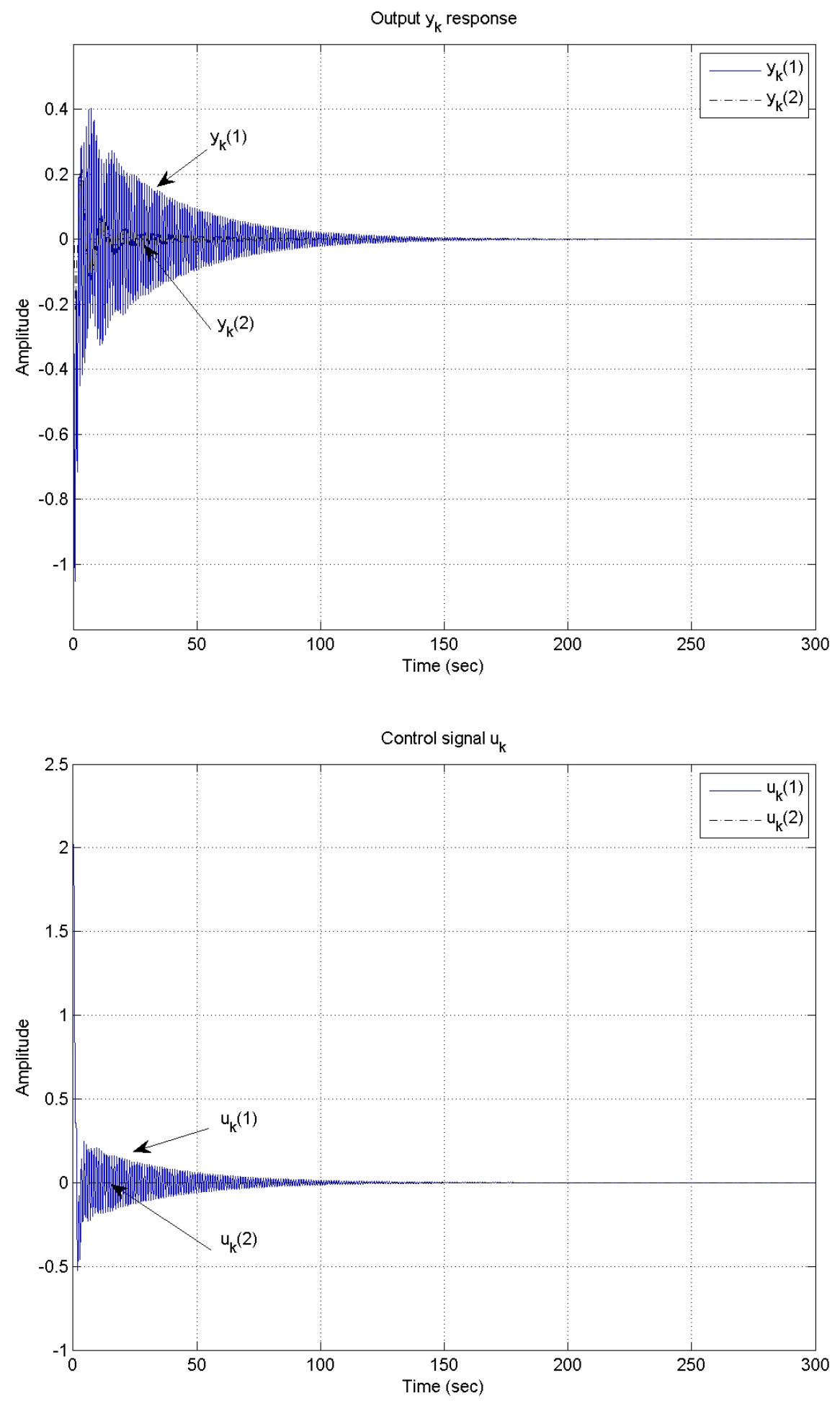

Figure 6.3-3: Turbo-Generator Example, Closed-Loop Response Two-Term Controller (2.6), $\theta=0$ with Controller Gains (6.4) Input $\eta_{0}=\left[\begin{array}{ll}1 & 0\end{array}\right]^{\top}$

Design 2 . 

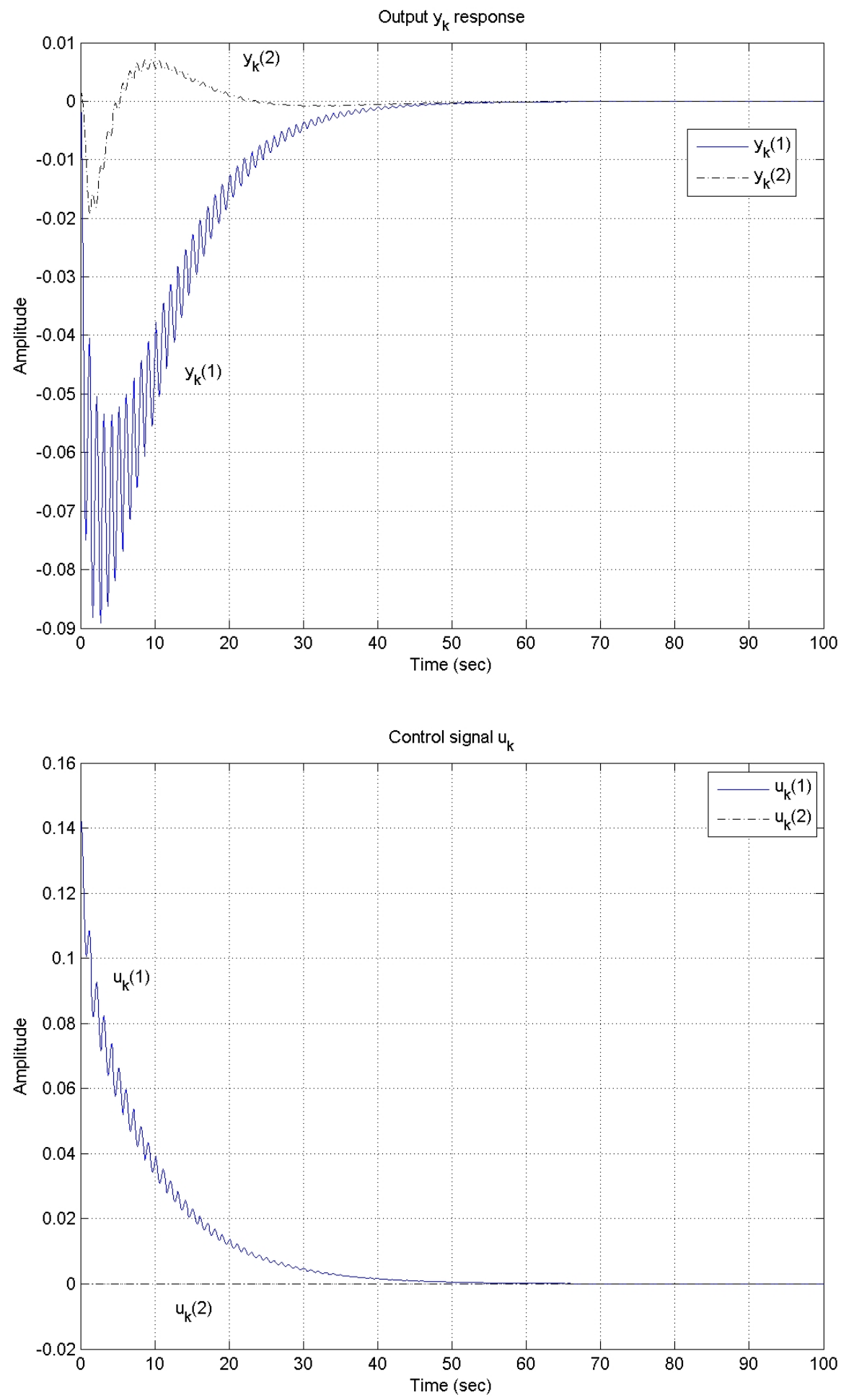

Figure 6.3-4: Turbo-Generator Example, Closed-Loop Response Two-Term Controller (2.6), $\theta=10$ with Controller Gains (6.5) (Better Controller) Input $\eta_{0}=\left[\begin{array}{ll}1 & 0\end{array}\right]^{\top}$

Design 2 . 

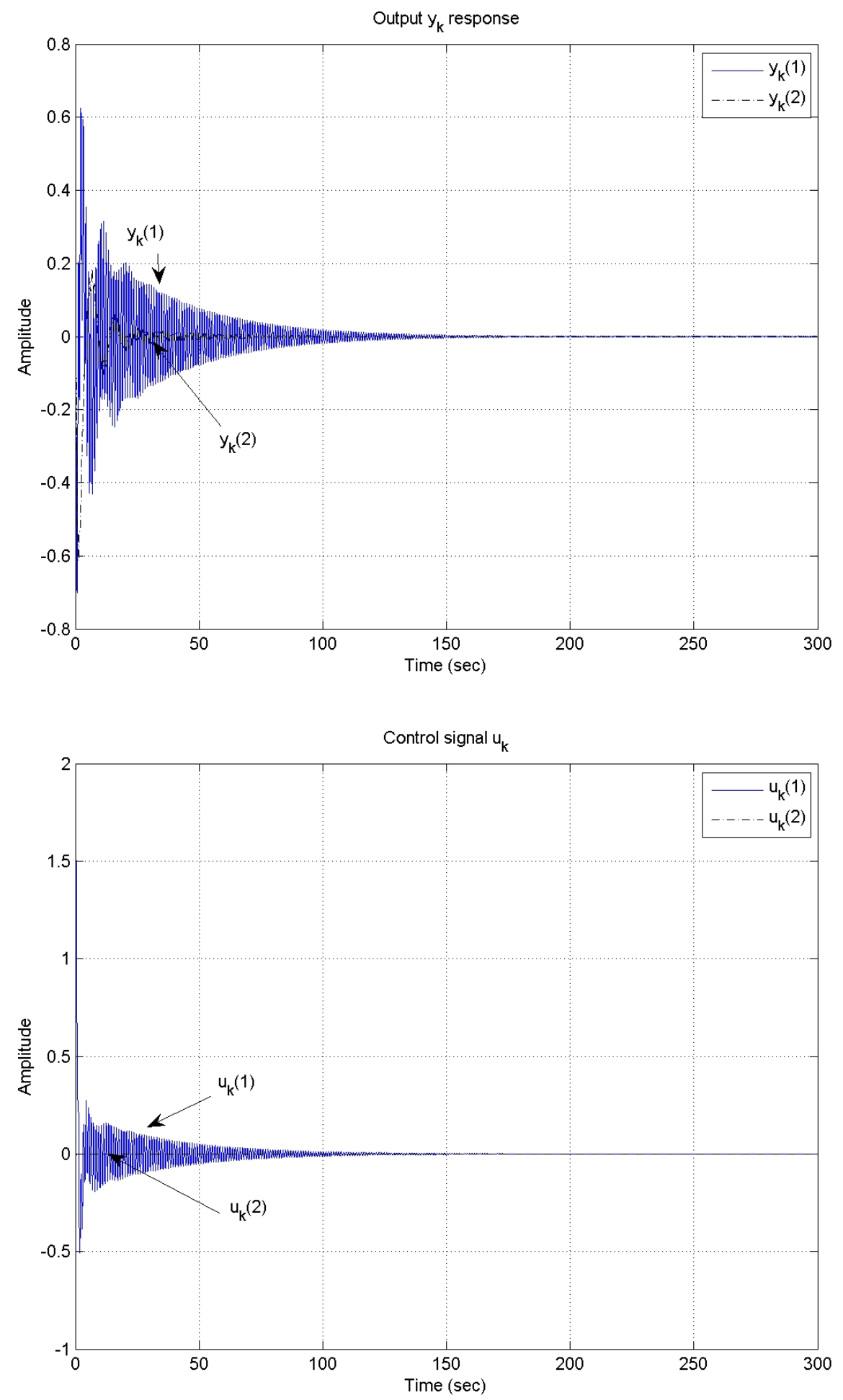

Figure 6.3-5: Turbo-Generator Example, Closed-Loop Response Two-Term Controller (2.6), $\theta=0$ with Controller Gains (6.4) Input $\eta_{0}=\left[\begin{array}{ll}0 & 1\end{array}\right]^{\top}$

Design 2 . 

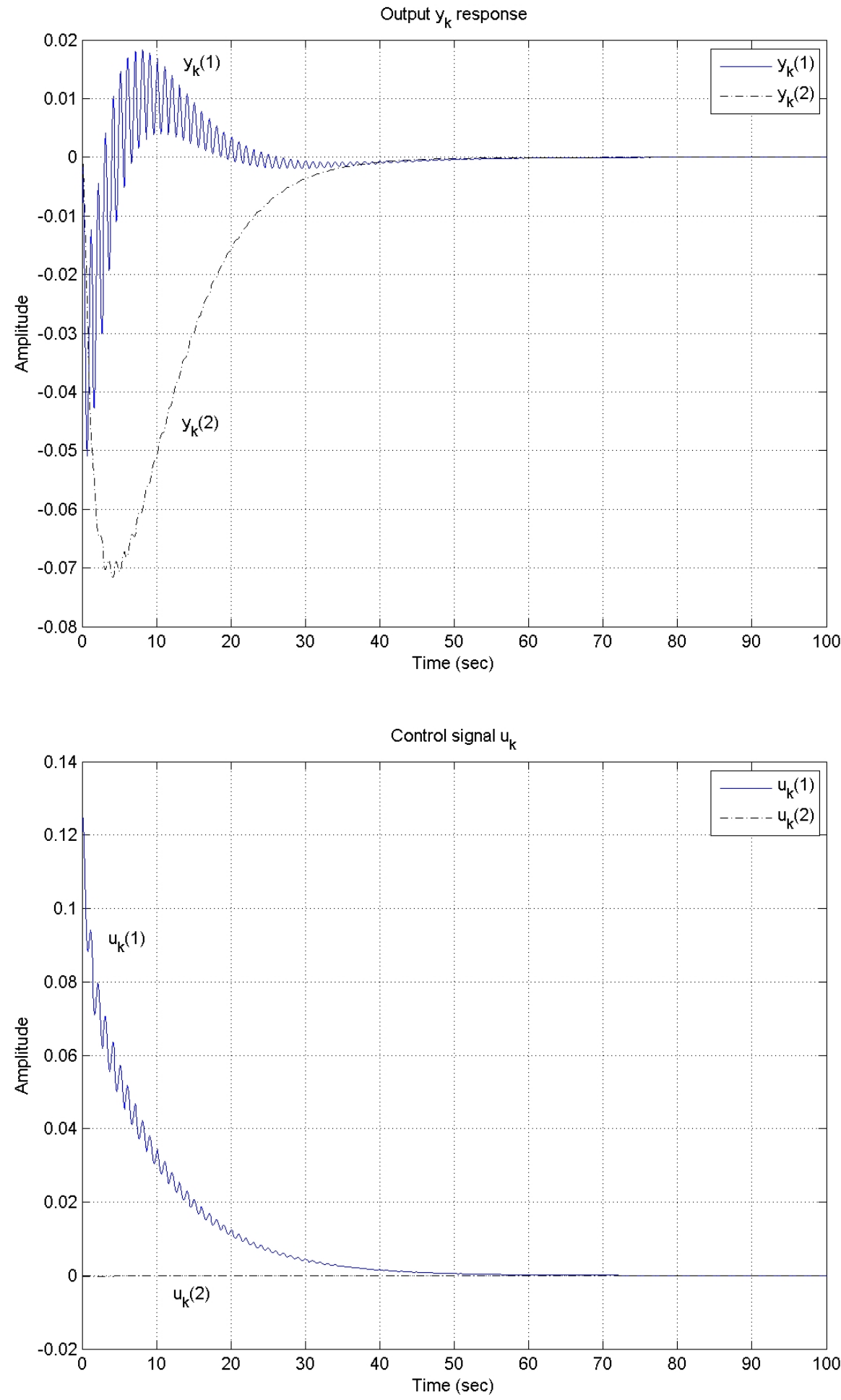

Figure 6.3-6: Turbo-Generator Example, Closed-Loop Response Two-Term Controller (2.6), $\theta=10$ with Controller Gains (6.5) (Better Controller) Input $\eta_{0}=\left[\begin{array}{ll}0 & 1\end{array}\right]^{\top}$

Design 2 . 


\subsection{Summary}

The proposed controller design approach is applied to a medium-scale system that is open-loop stable and nonminimum phase. During the design phase, the plant model is assumed to be unknown and there is no knowledge that the plant is nonminimum phase nor that it has a high resonance frequency with a low damping factor. By setting $\theta$ to be non-zero and $\mu$ to be sufficiently small in the performance indices $J_{A}(2.102)$ or $J_{B}$ (2.103), a relatively fast and smooth closed-loop output response is obtained for a given tracking reference input. 


\section{Chapter 7}

\section{Comparison Example}

In this example, we will compare our proposed controller design approach with the extremum seeking (ES) controller tuning scheme using one of the simulation examples presented in [13] with some modifications.

\subsection{Description of the Original Problem}

In [13], the objective is to tune the parameters of a PID controller of the form

$$
u(s)=K e(s)+\frac{K}{T_{i} s} e(s)+K T_{d} s y(s)
$$

for the plant

$$
G(s)=\frac{1}{1+20 s} e^{-5 s}
$$

using the performance index of the form

$$
J(\theta) \triangleq \frac{1}{T-t_{0}} \int_{t_{0}}^{T} e^{2}(t, \theta) \mathrm{d} t
$$

where $\theta \triangleq\left[\begin{array}{lll}K & T_{i} & T_{d}\end{array}\right]^{\top}$ and the cost $J(\theta)$ is calculated for each step-response experiment performed. The open-loop response of this system is given in Figure 7.1. The PID controller parameters given by the Ziegler-Nichols (ZN) tuning rules [3] are used as a starting point for the ES tuning, i.e., $\theta(0)=\left[\begin{array}{lll}4.06 & 9.25 & 2.31\end{array}\right]^{\top}$ in [13]. The cost function spans from $t_{0}=10 \mathrm{~s}$ to $T=100 \mathrm{~s}$, where $t_{0}$ is approximately the time at which the step response of the closed-loop system reaches the first peak under the controller gains given by ZN. This essentially puts zero weighting on the initial transient response. 
The ES tuning scheme is configured with adaptation gains $\gamma=\left[\begin{array}{lll}200 & 1200 & 200\end{array}\right]^{\top}$, perturbation amplitudes $\alpha=\left[\begin{array}{lll}0.1 & 1 & 0.1\end{array}\right]^{\top}$, dither frequencies $\omega=\left[\begin{array}{lll}0.8 \pi & 0.8^{2} \pi & 0.8^{3} \pi\end{array}\right]^{\top}$, and filter parameter $h=0.5[13]$.

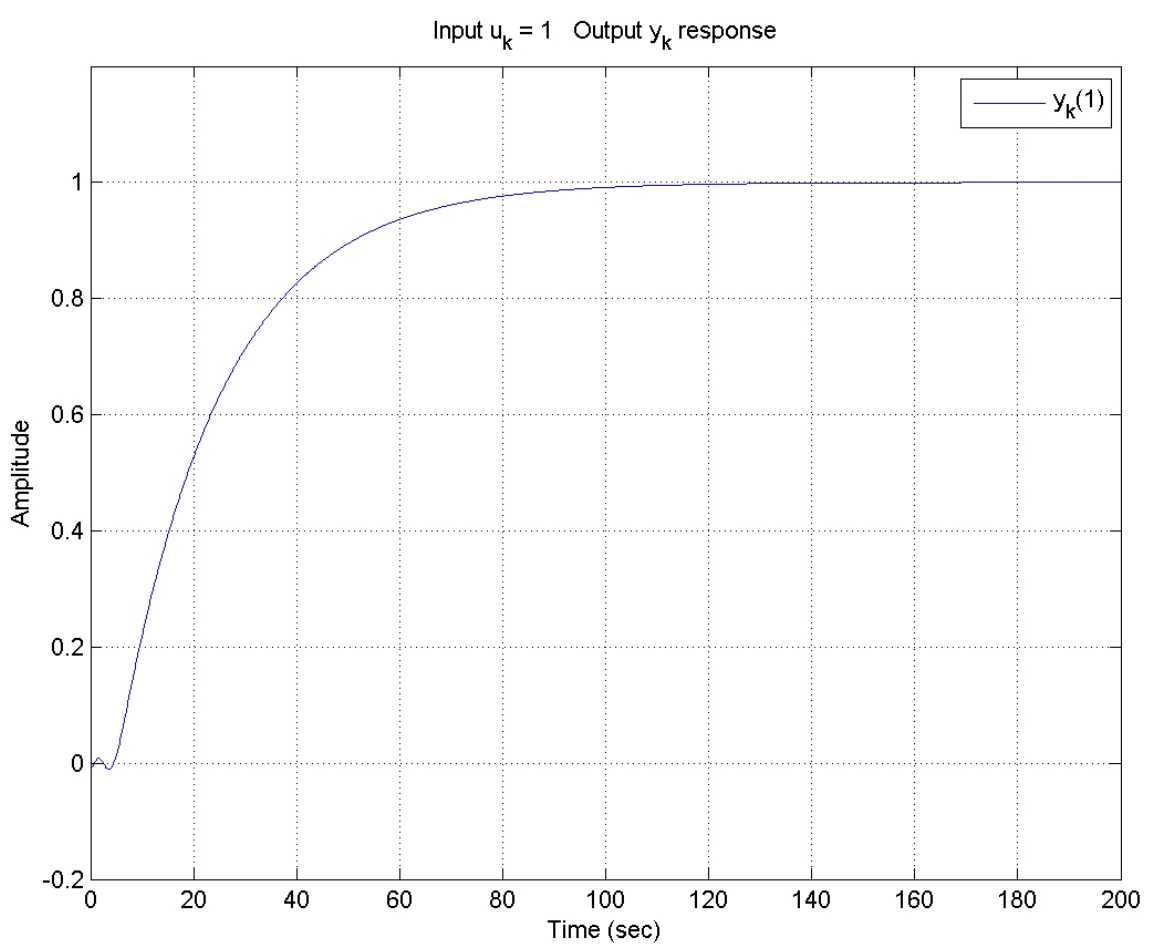

Figure 7.1: Comparison Example, Open-Loop Response. 


\subsection{Modifications to the Original Problem}

In order to be able to compare results obtained using ES tuning against those obtained using our proposed design approach with a two-term controller, we make the following modifications to the ES setup for the example presented in [13]:

- The PID controller is simplified to a PI controller of the form

$$
u(s)=K e(s)+\frac{K}{T_{i} s} e(s) .
$$

- The starting controller parameters for ES tuning are found to be $\theta(0)=\left[\begin{array}{ll}2.8 & 14.4\end{array}\right]^{\top}$ using the ZN method.

- The cost function now spans from $t_{0}=18 \mathrm{~s}$ to $T=100 \mathrm{~s}$, where $t_{0}$ is still approximately the time at which the step response of the closed-loop system reaches the first peak under the controller gains given by $\mathrm{ZN}$.

- The initial conditions of $\hat{\theta}(0)$ and $\zeta(0)$ in Equations (1.12) to (1.14) are set to be $\hat{\theta}(0)=\theta(0)$ and $\zeta(0)=J(\theta(0))$, as the values for $\hat{\theta}(0), \zeta(0)$ were not given in the original example [13]. 


\subsection{Control Design using Extremum Seeking}

The PI controller (7.4) is obtained by minimizing the performance index (7.3). The optimization is carried out under the ES parameter settings of:

$$
\gamma=\left[\begin{array}{c}
200 \\
1200
\end{array}\right], \alpha=\left[\begin{array}{c}
0.1 \\
1
\end{array}\right], \omega=\left[\begin{array}{c}
0.8 \pi \\
0.64 \pi
\end{array}\right], \text { and } h=0.5 ;
$$

in this case the optimal control parameters $\theta=\left[\begin{array}{ll}K & T_{i}\end{array}\right]^{\top}$ are found to be

$$
K=1.9351, \quad T_{i}=21.0554 .
$$

Simulation of the tracking reference input is given in Figure 7.2 for the resulting closedloop system. Figures 7.3-1 to 7.3-4 illustrate the impact of the choice of ES parameters on the stability and convergence rate of the ES tuning scheme.

Remark 7.1. It is seen that ES converges at different rates depending on the choice of $\gamma, \alpha$ and $\omega$ values. There are also cases where ES diverges and causes the overall closed-loop system to become unstable. For example, setting $\zeta(0)=0.5 J(\theta(0))$ or $\alpha=\left[\begin{array}{ll}0.4 & 4\end{array}\right]^{\top}$ destabilizes the system. Furthermore, if the starting controller parameters $\theta(0)=\left[\begin{array}{ll}2.8 & 14.4\end{array}\right]^{\top}$ are changed to $\theta(0)=\left[\begin{array}{ll}0.28 & 14.4\end{array}\right]^{\top}$, the ES tuning scheme does not converge. The domain of attraction can be increased by decreasing $\gamma$ at the cost of a slower convergence rate.

Remark 7.2. In the simulation results presented, ES converges after several hundreds of function evaluations or more. This amounts to a long period of time to simulate the ES tuning process using the simulation procedure described in Chapter 3.3. In order to demonstrate the need of increasing the perturbation amplitude $\alpha$ when the plant is subjected to noise, the ES tuning example presented would need to be repeated starting with smaller $\alpha$ values. However, this also means that the convergence rate is much slower and much more time is required for simulation. As a result, noise sensitivity analysis via Simulink simulations was not performed. 

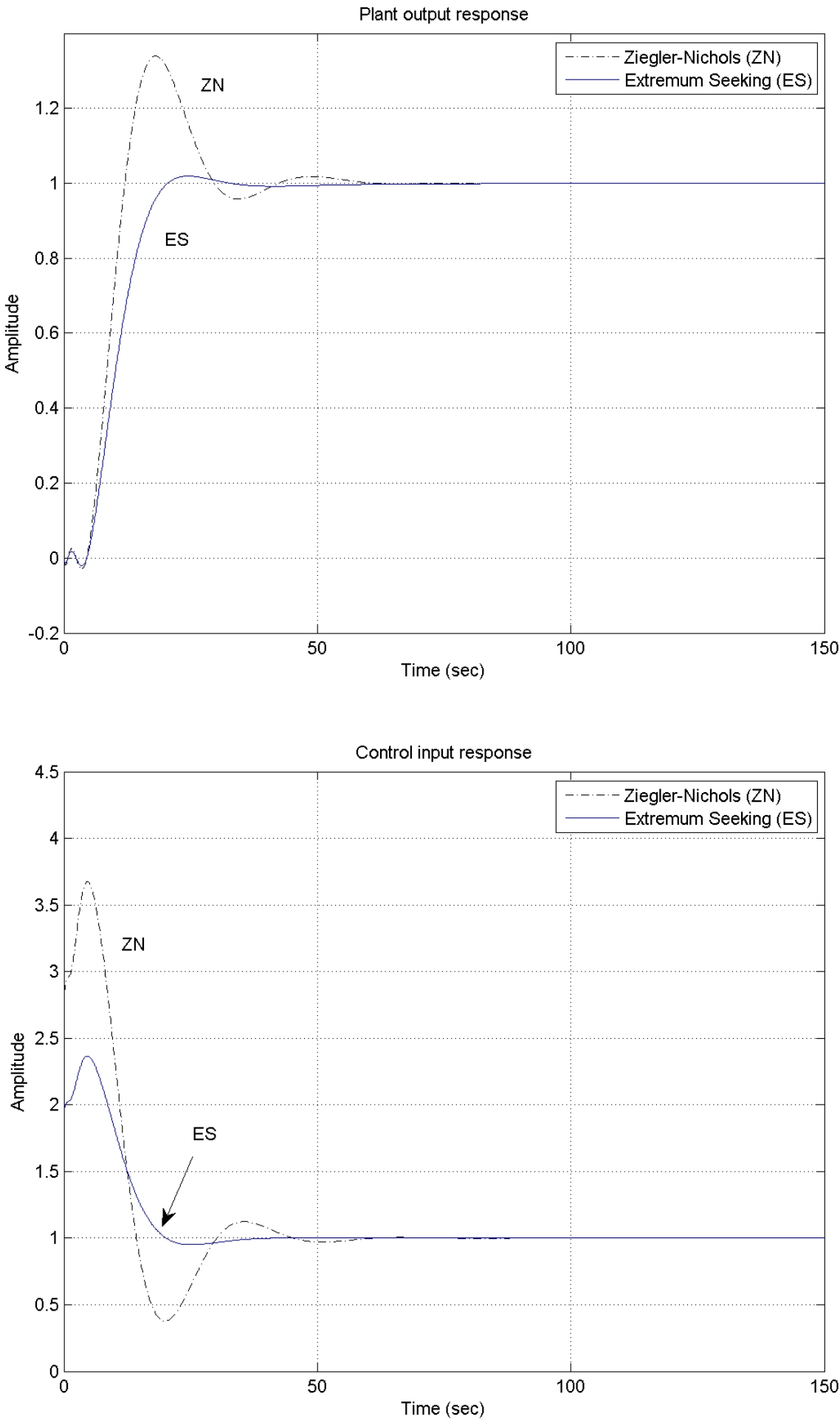

Figure 7.2: Comparison Example, Closed-Loop Response PI Controller

Input $y_{\text {ref }}=1$. 

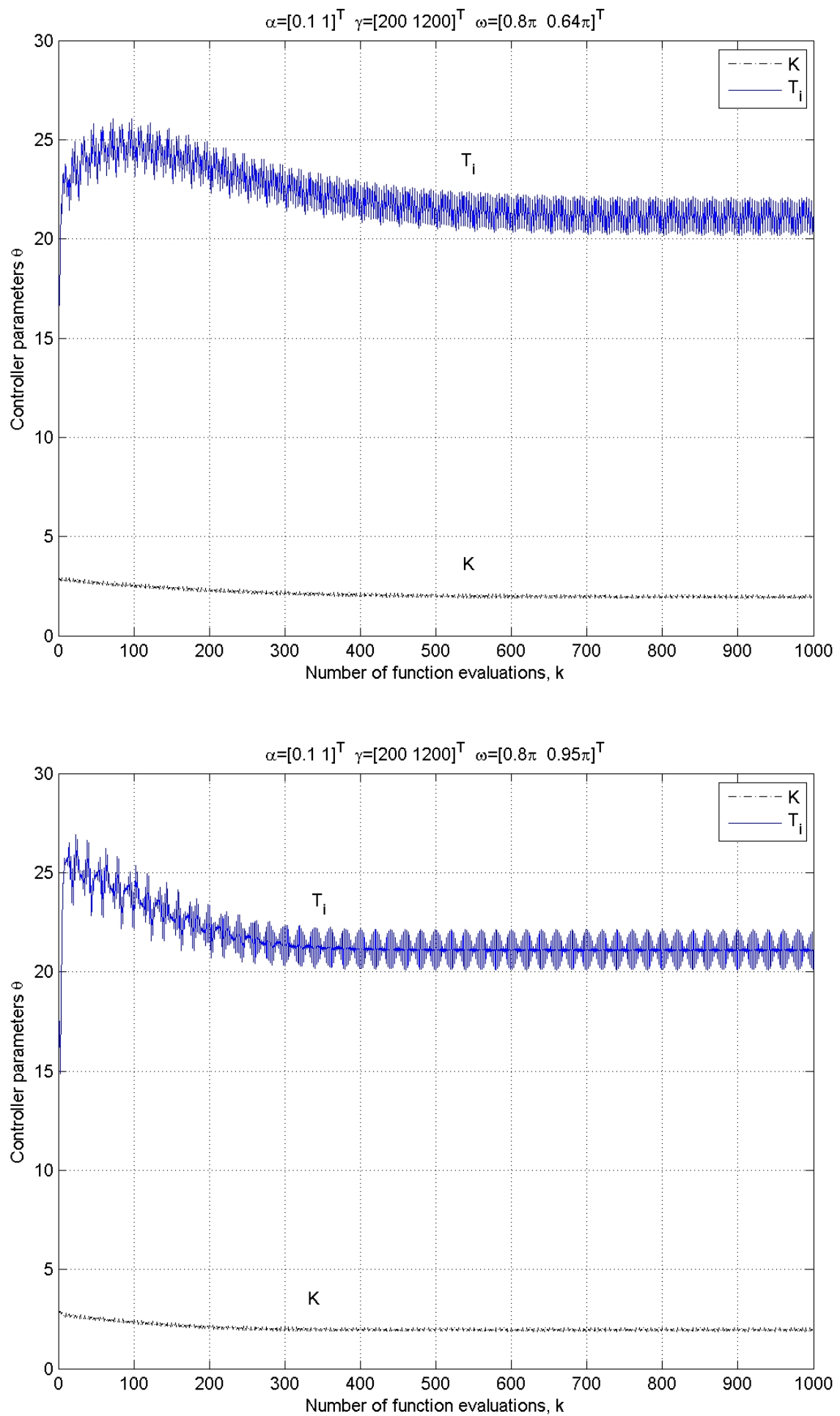

Figure 7.3-1: Comparison Example, Evolution of PI Parameters. 

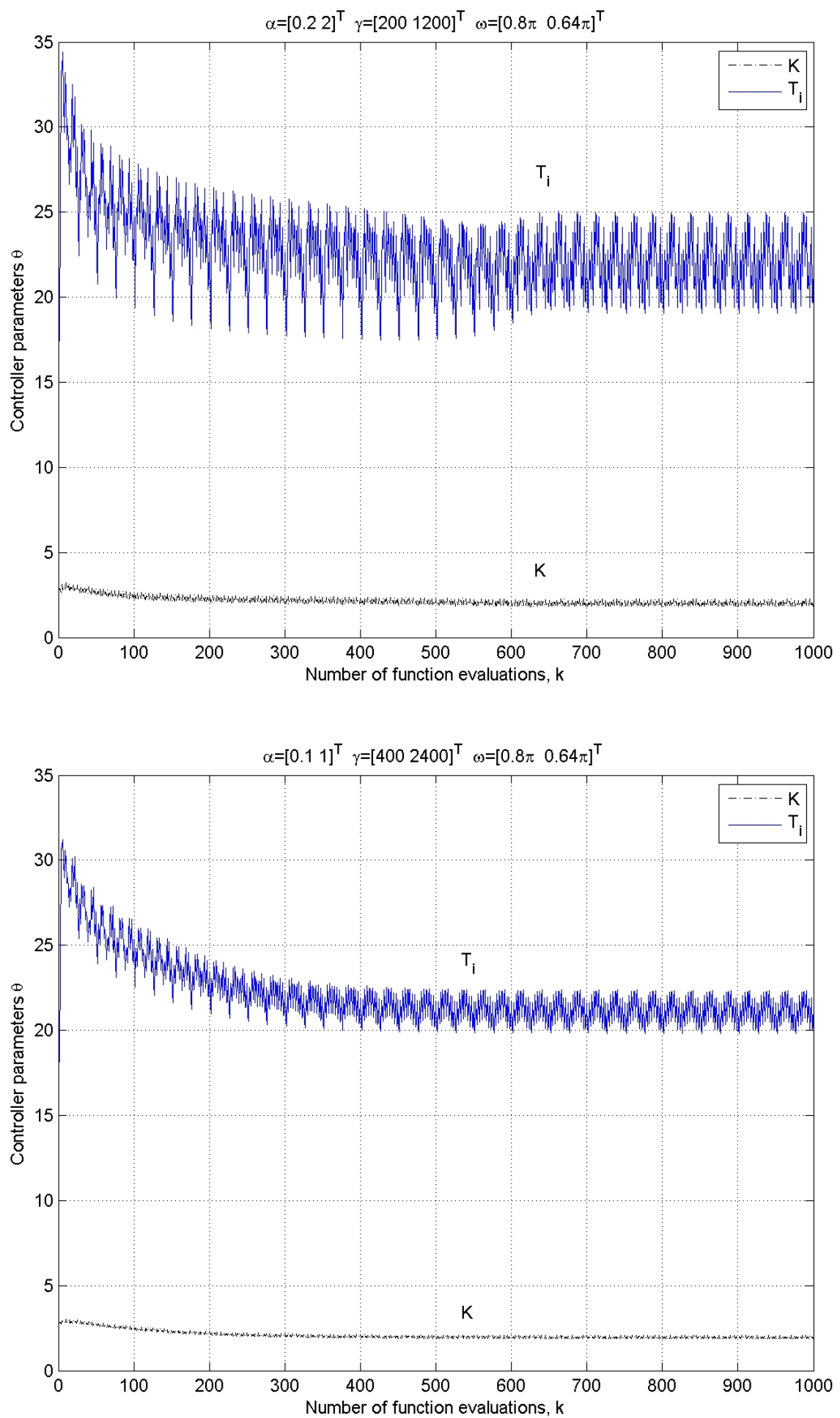

Figure 7.3-2: Comparison Example, Evolution of PI Parameters. 

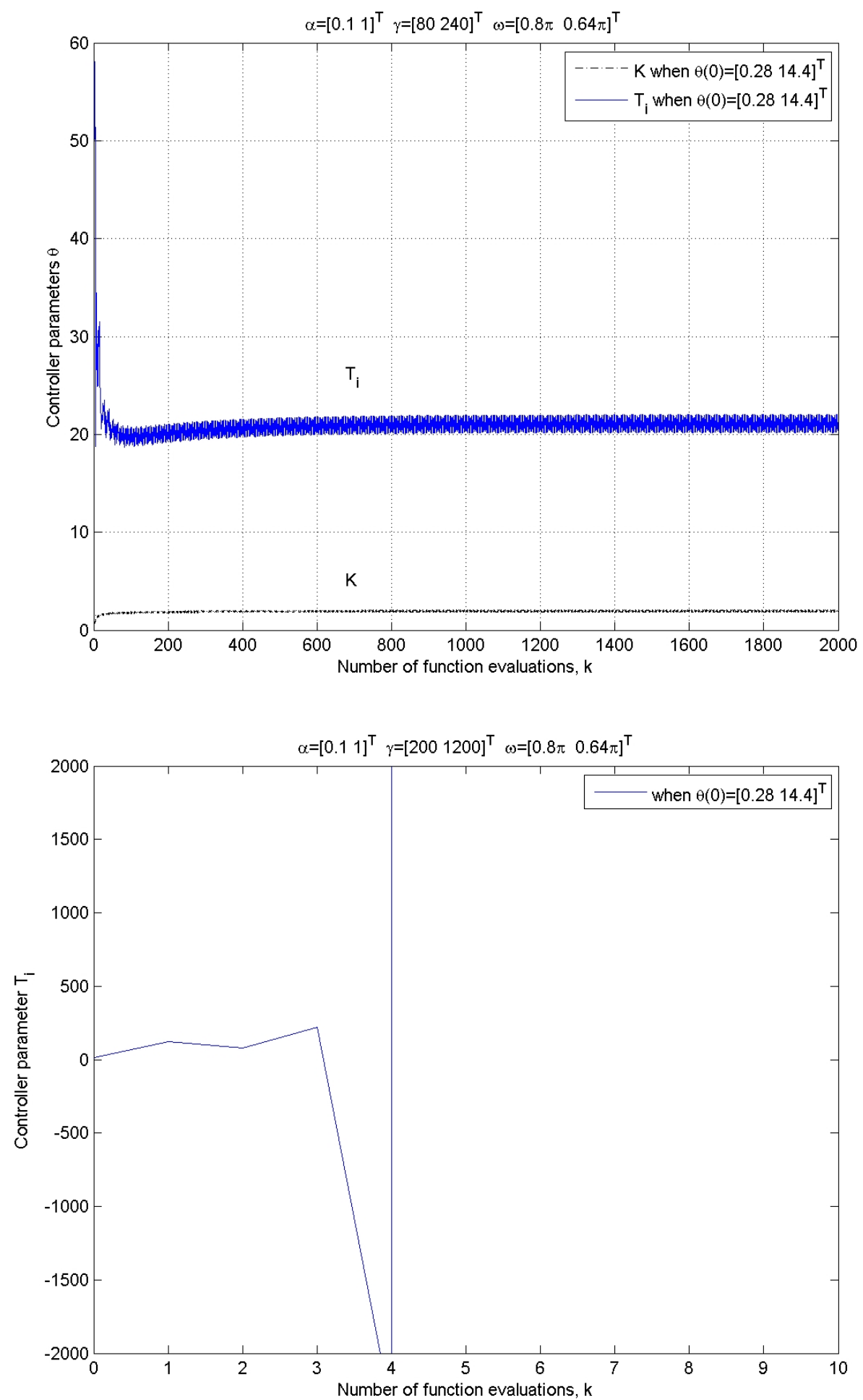

Figure 7.3-3: Comparison Example, Evolution of PI Parameters. 

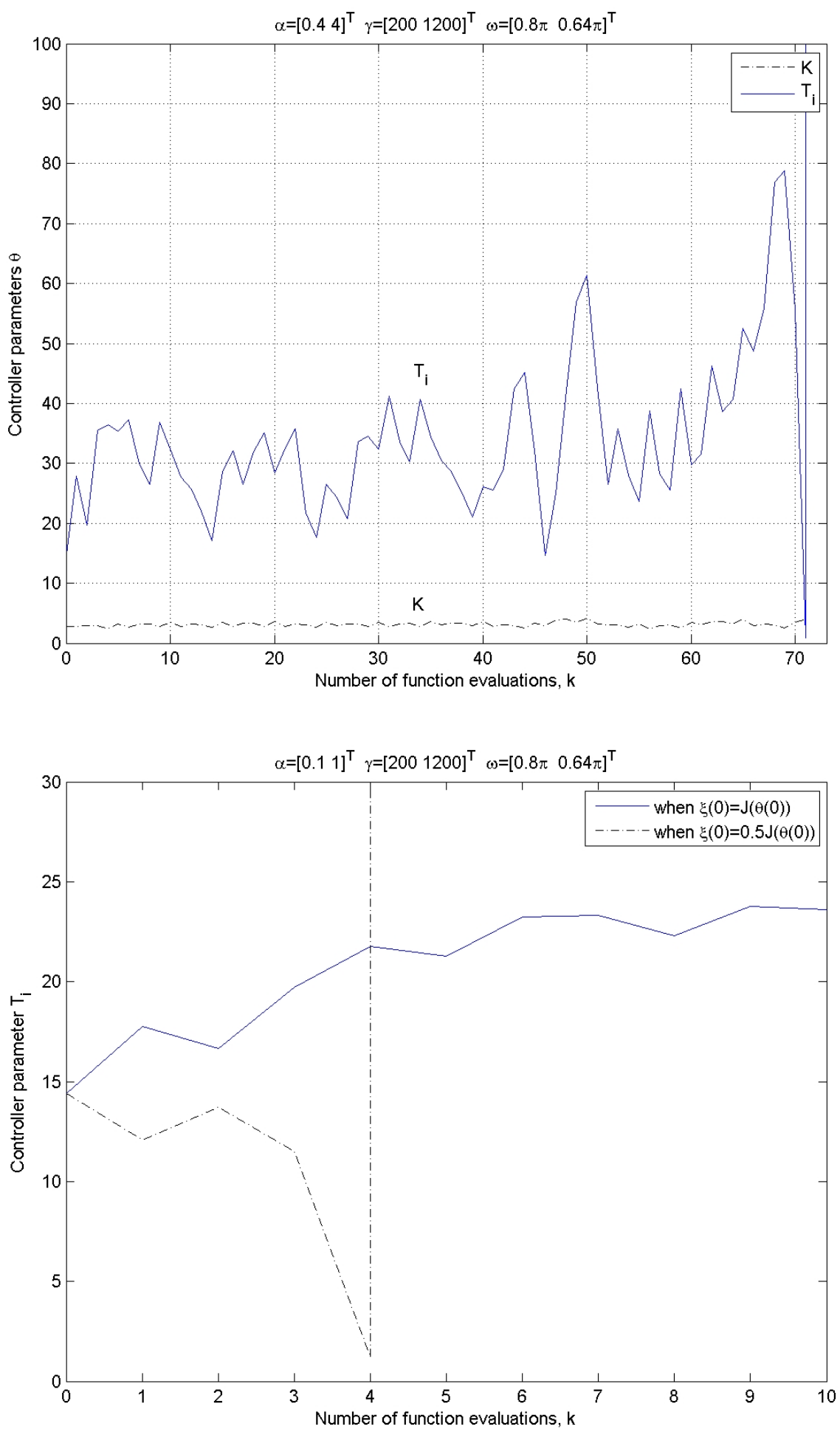

Figure 7.3-4: Comparison Example, Evolution of PI Parameters. 


\subsection{Control Design using Proposed Approach}

In order to be able to compare the proportional and integral gains obtained using our proposed design approach against those obtained using the ES tuning, we modify our two-term controller (2.6) to become

$$
u_{k}=-S\left(K_{P} e_{k}+K_{I} \eta_{k}\right)
$$

To design the controller (7.6), we first sample $G(s)$ with a sampling period of $h=0.1$ seconds and implement the time delay by using a $3^{\text {rd }}$ order Padé approximation. The sampling period is chosen to be sufficiently small so that our discrete domain results can be compared with the continuous domain results obtained in Section 7.3 using extremum seeking. The steady-state tracking matrix $\Gamma(2.2)$ for plant $G(s)(7.2)$ is obtained experimentally to be $\Gamma=1$. The two-term controller is then obtained by minimizing performance index $J_{A y_{r e f}}$ given in (2.59) using $\theta=0, \mu=10^{-8}$. The optimization is carried out under the system settings of:

$$
x_{0}=0, \eta_{0}=0, \text { and } y_{r e f}=1
$$

in this case the optimal controller gains $K_{P}$ and $K_{I}$ are found to be

$$
\text { Case } t_{0}=0 s: \quad K_{P}=3.2519, \quad K_{I}=1.0581 \times 10^{-1},
$$

or equivalently,

$$
\text { Case } t_{0}=0 s: \quad K=K_{P}=3.2519, \quad T_{i}=\frac{K_{P}}{K_{I}}=3.0732 \times 10^{1} .
$$

If we modify the summation boundaries of the performance index $J_{A}(2.102)$ such that it corresponds to a time span from $t_{0}=10 \mathrm{~s}$ to $T \approx \infty$, we obtain

$$
\text { Case } t_{0}=10 s: \quad K=2.6821, \quad T_{i}=2.5596 \times 10^{1} .
$$

If the cost function is further modified to span from $t_{0}=18 s$ to $T \approx \infty$, the optimal gains are found to be

$$
\underline{\text { Best Controller }} \text { Case } t_{0}=18 s: \quad K=1.9489, \quad T_{i}=2.1151 \times 10^{1} .
$$

Simulation of the tracking reference input is given in Figure 7.4 for the resulting closedloop system. A summary of the optimization results is given in Tables 7.1 to 7.3 , and the detailed optimization output can be found in Appendix C.3. 
Remark 7.3. There is a slight discrepency between the optimal gains obtained using our proposed method and those obtained using the sinusoidal perturbation-based extremum seeking scheme. Under extremum seeking, the controller gains locally exponentially converge to an $O(\alpha)$ neighborhood of the true optimal gains, where $\alpha$ is the perturbation amplitude [10]. We will, however, treat the optimal gains obtained using the two different methods to be identical.

Remark 7.4. Even though a "truncated" cost function, which places zero weighting on the initial transient response, gives rise to a faster system response with smaller overshoot, choosing a good value for the starting time $t_{0}$ is not straightforward. Unlike the ES tuning method, changing the time span of the performance index or performing the optimization from a new set of initial controller gains did not require any changes in our setup. We obtained the same optimal controller gains and closed-loop response as those obtained using ES tuning but with a much simpler setup.

\begin{tabular}{ccccccc}
\hline Initial & Initial & Final & Final & Initial & Final & $\begin{array}{c}\text { Number of } \\
\text { function } \\
K_{P}\end{array}$ \\
$K_{I}$ & $K_{P}$ & $K_{I}$ & cost $J$ & cost $J$ & $\begin{array}{c}\text { coluations } \\
\text { evaluation }\end{array}$ \\
\hline $5.00 \times$ & $1.00 \times$ & & $1.06 \times$ & & & 126 \\
$10^{-3}$ & $10^{-3}$ & 3.25 & $10^{-1}$ & 510.06 & 7.53 & \\
\hline
\end{tabular}

Table 7.1: Summary of controller parameter optimization for the SISO time-delayed system with the cost function spanning from $t_{0}=0 s$ to $T \approx \infty$.

\begin{tabular}{ccccccc}
\hline Initial & Initial & Final & Final & Initial & Final & $\begin{array}{c}\text { Number of } \\
\text { function } \\
K_{P}\end{array}$ \\
$K_{I}$ & $K_{P}$ & $K_{I}$ & cost $J$ & cost $J$ & $\begin{array}{c}\text { coluations } \\
\text { evaluation }\end{array}$ \\
\hline $5.00 \times$ & $1.00 \times$ & 2.68 & $\begin{array}{c}1.05 \times \\
10^{-1}\end{array}$ & 500.07 & $\begin{array}{c}2.40 \times \\
10^{-1}\end{array}$ & 118 \\
\hline $10^{-3}$ & $10^{-3}$ & & & & \\
\hline
\end{tabular}

Table 7.2: Summary of controller parameter optimization for the SISO time-delayed system with the cost function spanning from $t_{0}=10 \mathrm{~s}$ to $T \approx \infty$. 


\begin{tabular}{ccccccc}
\hline Initial & Initial & Final & Final & Initial & Final & $\begin{array}{c}\text { Number of } \\
\text { function } \\
K_{P}\end{array}$ \\
$K_{I}$ & $K_{P}$ & $K_{I}$ & $\operatorname{cost} J$ & $\begin{array}{c}\text { cost } J \\
\text { evaluations }\end{array}$ \\
\hline $1.00 \times$ & $1.00 \times$ & 1.95 & $\begin{array}{c}9.21 \times \\
10^{-2}\end{array}$ & 494.59 & $\begin{array}{c}4.49 \times \\
10^{-3}\end{array}$ & 175 \\
\hline $10^{-4}$ & $10^{-3}$ & & & & & \\
\hline
\end{tabular}

Table 7.3: Summary of controller parameter optimization for the SISO time-delayed system with the cost function spanning from $t_{0}=18 \mathrm{~s}$ to $T \approx \infty$. 

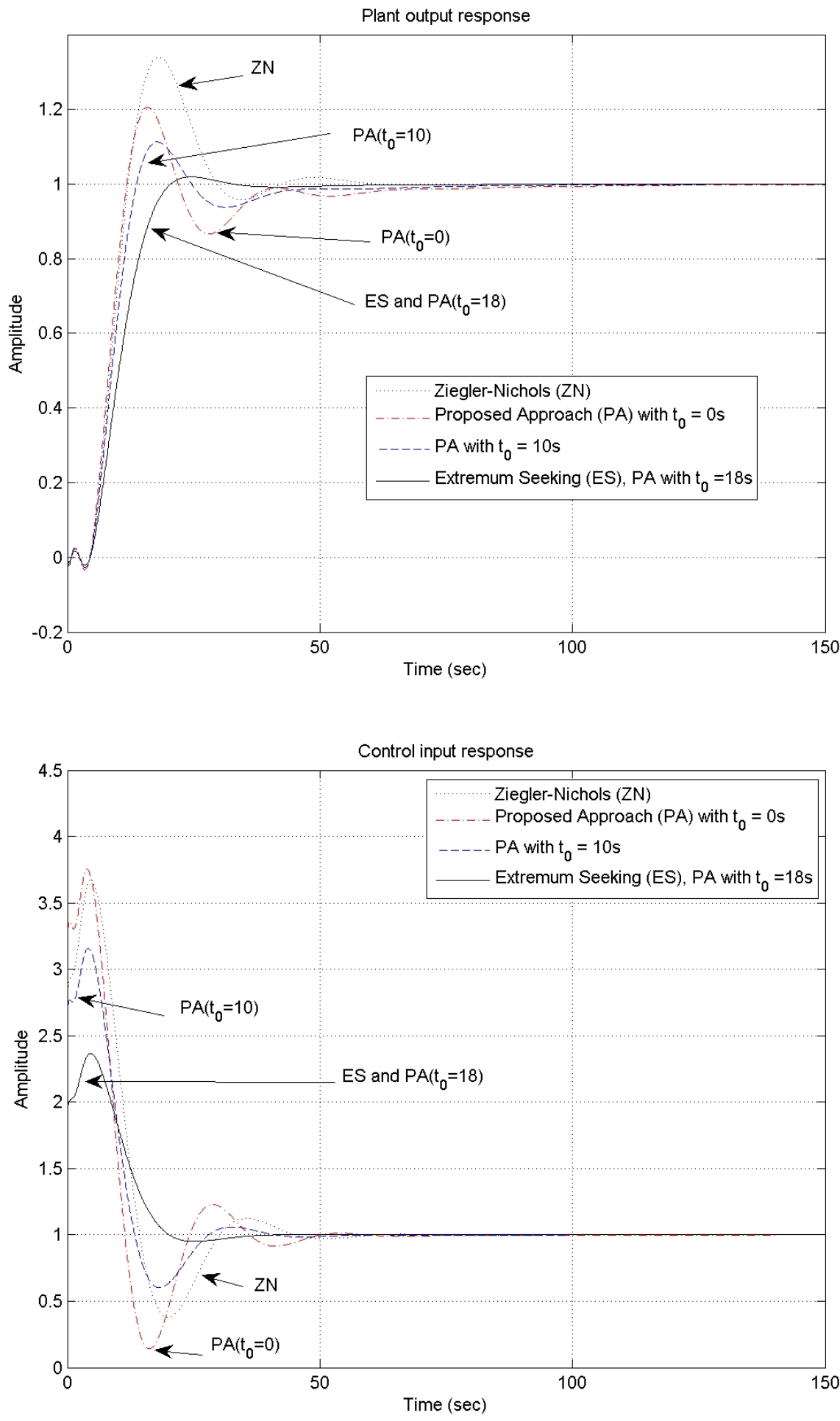

Figure 7.4: Comparison Example, Closed-Loop Response PI Controller

Input $y_{\text {ref }}=1$. 


\subsection{Summary}

The proposed controller design approach is applied to a single-input single-output system with time delay. During the design phase, no knowledge of the plant model or presence of time delay was required. Even though the "Window" cost function used in ES tuning gives rise to a response with the smallest overshoot and fastest settling time, there are no guarantees for bounded peaking of the state trajectories or the stability of the closed-loop system. If a different plant, performance index or starting controller gains is of interest, one would have to maintain the stability of ES through the careful selection of the ES parameters. Unlike ES, our proposed method is more "robust" in the sense that stability is maintained for every non-negative $\theta$ and positive $\mu$ values in our performance indices. 


\section{Chapter 8}

\section{MARTS Experiment}

The purpose of this chapter is to validate, experimentally, the effectiveness of the proposed controller design approach via the use of an experimental test bed known as MARTS. A description of MARTS and the details of the experimental setup are initially provided. The process of developing linear control valve characteristics, measuring steady-state tracking matrix and setting up the optimization problem on MARTS are also described. Thereafter, we concentrate on the experimental results.

\subsection{Description of MARTS}

The experimental test bed is called MARTS(Multivariable Apparatus for Real Time Systems) [42] and is composed of two interconnected vessels, a network of pipes and valves, and a variety of industrial commercial process-control sensors and actuators that monitor/control a hydraulic dynamic process. The setup which we are concerned with in this thesis is shown in Figure 8.1. The objective in using this configuration is to find a controller to control the position of the water level in both vessels by using only the two control valves. The controller design process can be challenging due to nonlinear effects of the plant, time delays, actuator valve dynamics, as well as actuator saturation and sizing effects, and interaction effects when the interconnecting valve 1 linking the two vessels is "widely" open.

In Figure 8.1, the control inputs to the plant are valve control voltages for the two control valves ( $-5 \mathrm{~V}$ corresponds to fully closed and $5 \mathrm{~V}$ corresponds to fully opened) that affect the amount of water inflow into the two vessels. The outputs of the plant are the water height measurements in the two water vessels via sensors which provide 
voltage levels ( $1 \mathrm{~V}$ corresponds to near empty and $5 \mathrm{~V}$ corresponds to near full, with $1 \mathrm{~V}$ increase/decrease representing approximately $2.4 \mathrm{~L}$ of water rise/drop or a $30 \mathrm{~cm}$ change in water level height). There are two disturbance inputs to the plant that provide unmeasurable water inflow disturbances via digital on/off control inputs. To perform real-time control, a Windows-based PC running MATLAB Version 7.2.0.232(R2006a) with the Real-Time Windows Target software is inserted within the control loop. Furthermore, the online optimization process is carried out using the combination of MATLAB and Simulink as described in Section 3.3, where the two-term controller (2.6) is implemented in Simulink and the Nelder-Mead algorithm is executed in MATLAB. A photograph of the experimental setup is shown in Figure 8.2, and a full list of components used within the experiment can be found in [43].

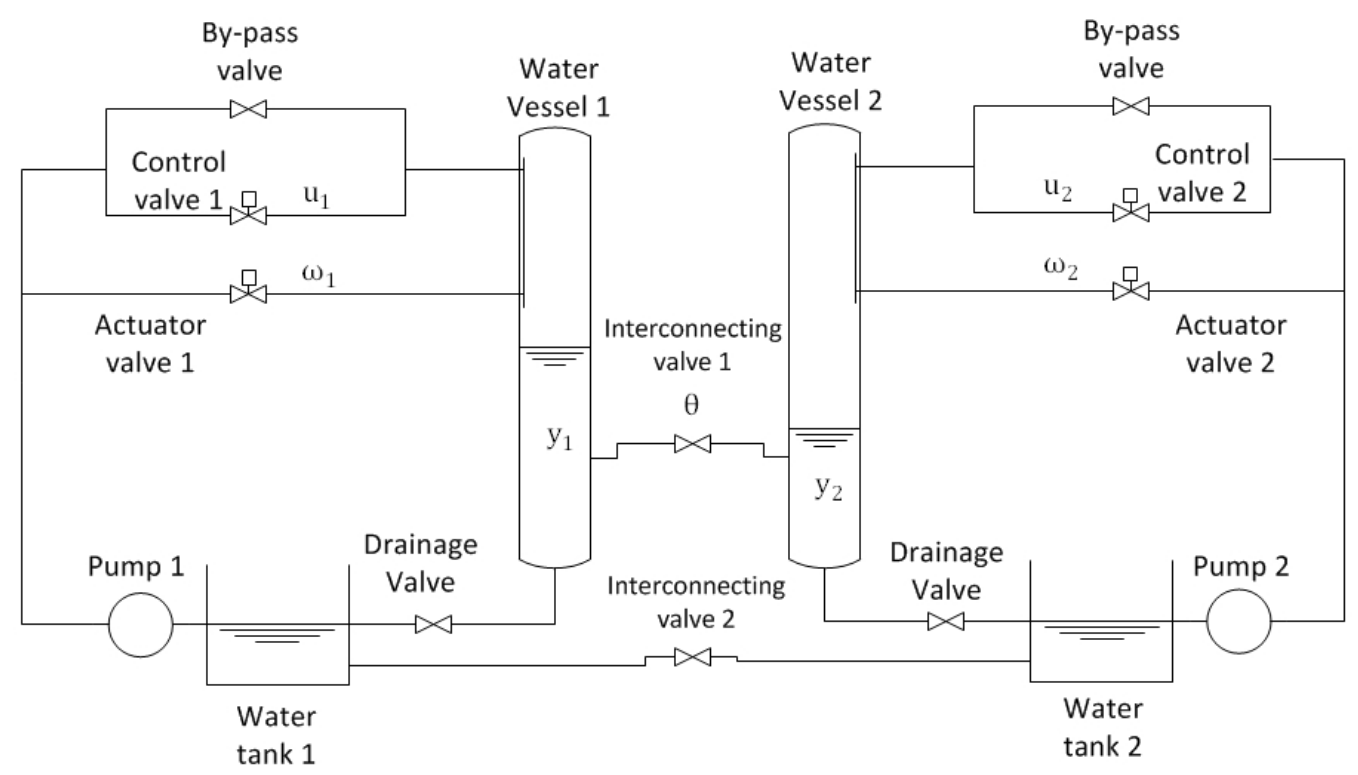

Figure 8.1: Schematic of MARTS setup. 


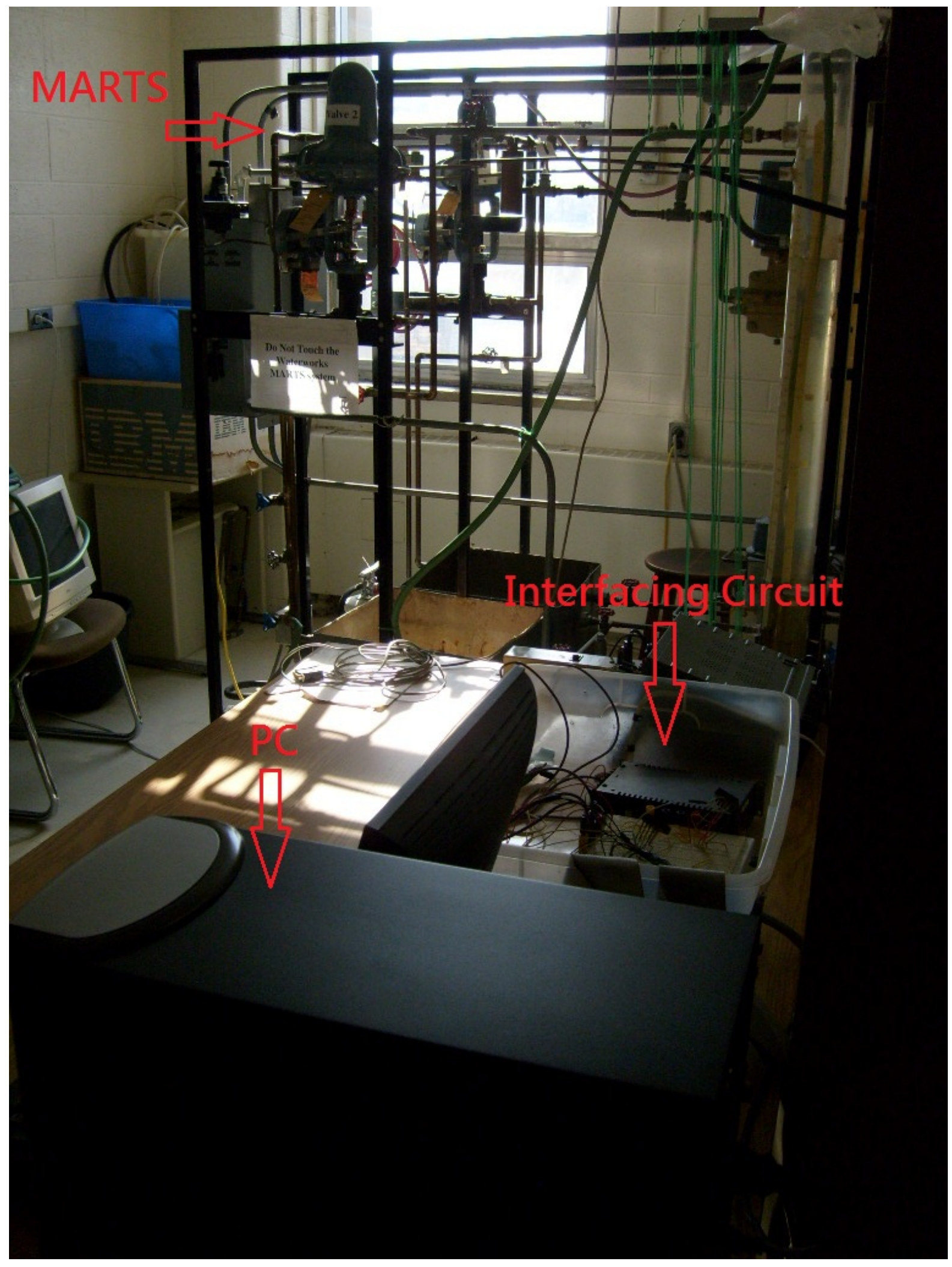

Figure 8.2: MARTS experimental setup. 


\subsection{Developing Linear Control Valve Characteristics}

The control valves on MARTS exhibit the non-linear characteristics as shown in Figure 8.3-1. In an attempt to "remove" the nonlinear valve characteristics, a square root function of the form

$$
u=\alpha \sqrt{(\hat{u}+5)}-5
$$

is applied, where $\hat{u}$ is the output of a controller and $u$ is the valve control voltage. $\alpha$ is chosen to be 2.8 for control valve 2 , and no compensation was done for control valve 1 since a better linear fit can only be obtained, using the square root function, at the cost of reducing the maximum flow rate by $50 \%$. The linearized valve characteristics are shown in Figure 8.3-2. A $1 V$ change in the control input now corresponds to a change of approximately $0.0072 \mathrm{~L} / \mathrm{s}$ in flow rate for control valve 1 and $0.0108 \mathrm{~L} / \mathrm{s}$ for control valve 2 . 

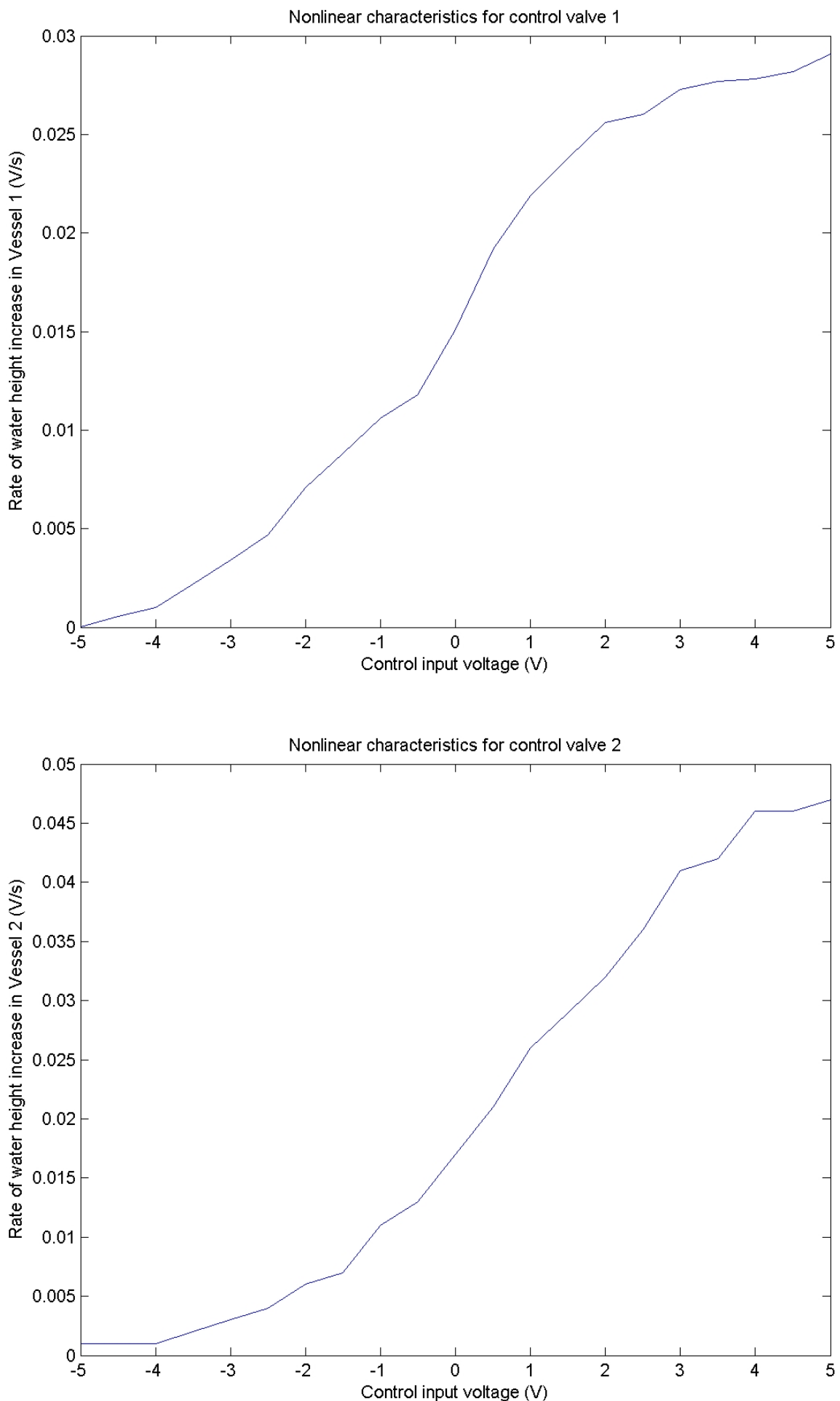

Figure 8.3-1: Nonlinear valve characteristics obtained experimentally. 

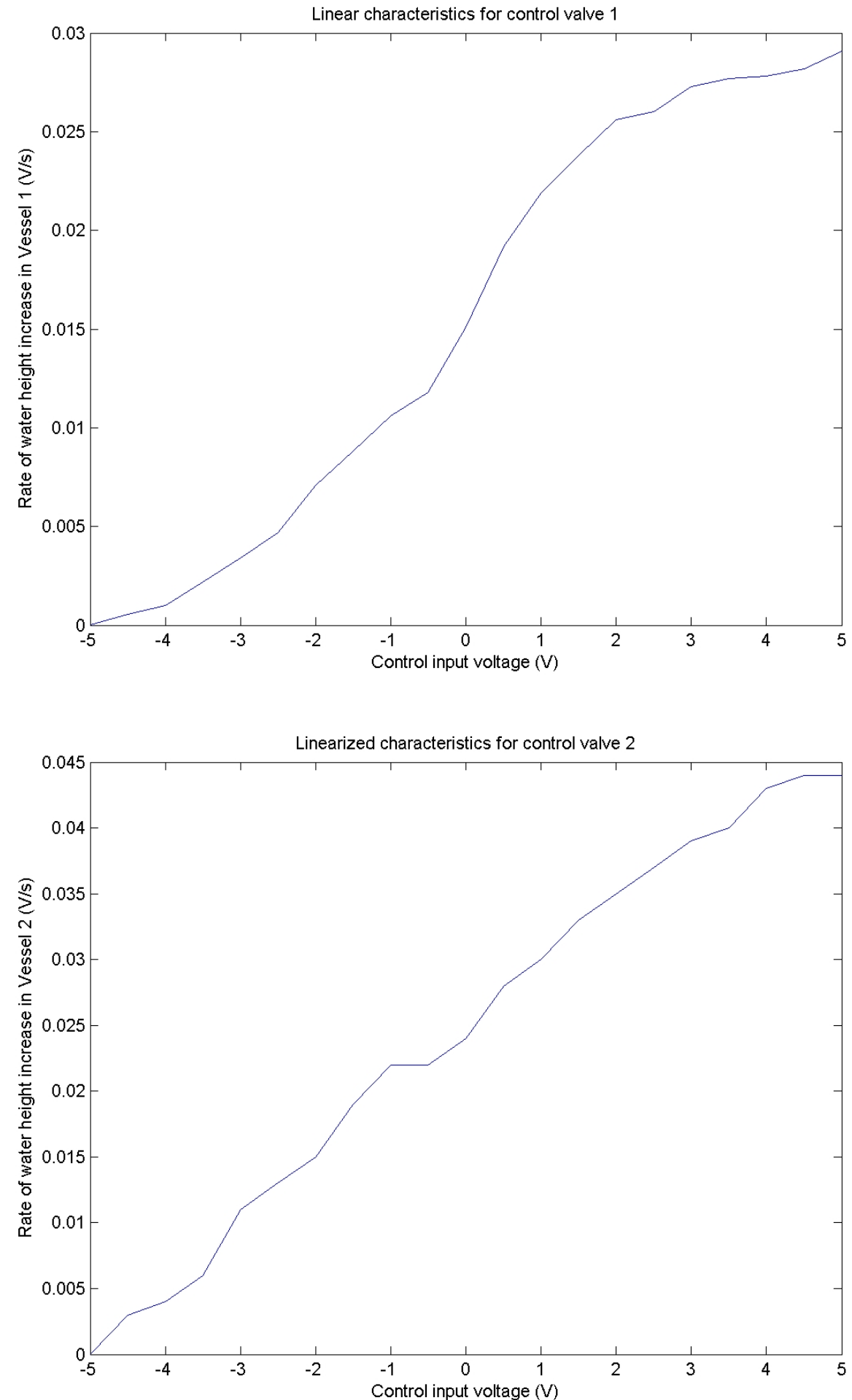

Figure 8.3-2: Linearized valve characteristics obtained experimentally. 


\subsection{Unexpected Observation}

Figure 8.4 shows the plant output over a 12 hours period with control valve inputs fixed at $0 \mathrm{~V}$. The water inflow and outflow rates were initially balanced by manually adjusting the drainage and by-pass valves. Due to the fact that the system is not openloop asymptotically stable (but is only stable), one would expect the water level in the vessels to remain at constant values or possibly to "drift" somewhat over a 12 hours period; however, this was not the case as shown in Figure 8.4 with the appearance of non-stationary saw-tooth like waveforms. The behavior observed in the two water vessels are different from each other, and we suspect that it may be due to various aging effects in the pumps and/or control valves; thus it was observed that the amount of control effort required to maintain the water level at a specific height changes overtime. Combined with the high frequency signal noise that arises as a result of water splashes and quantization noise, the saw-tooth like waveform poses difficulties in accurately comparing costs of different controllers. As a work around solution, we can

1. enlarge the initial Nelder-Mead simplex so that the performance of the various controllers being compared can be distinguished from each other, and

2. re-adjust the by-pass and drainage valves such that the water inflow and outflow rates are balanced at the desired water level height upon each restart of the NelderMead algorithm. 

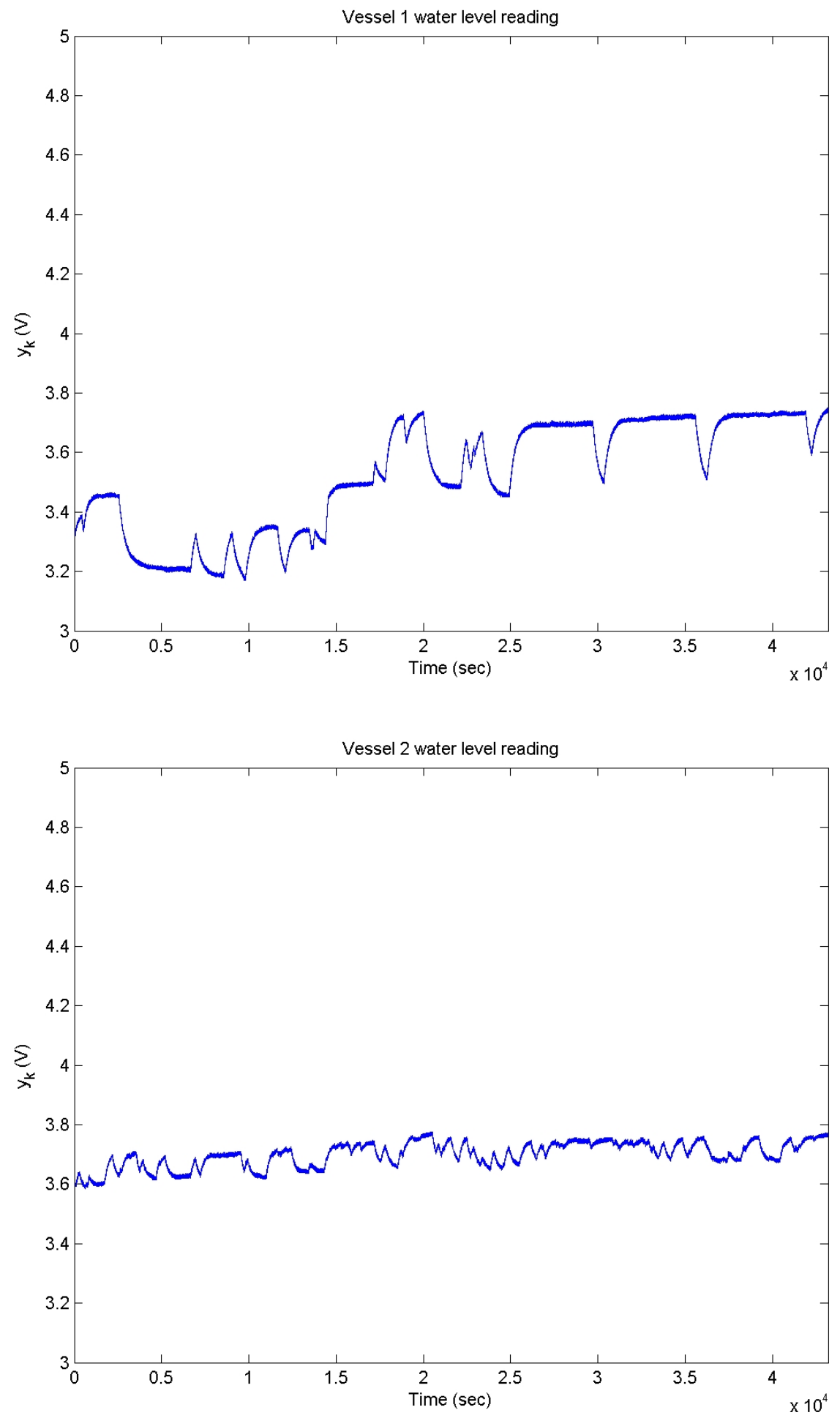

Figure 8.4: A 12 hour record of water level measurements with zero input flow applied to the two vessels. 


\subsection{Measuring the Steady-State Tracking Matrix}

MARTS is an open-loop stable plant (but not asymptotically stable) under the configuration shown in Figure 8.1. Thus to stabilize the system, we precondition the system with a stabilizing output feedback $u_{k}(1)=-10 y_{k}(1)$ and $u_{k}(2)=-10 y_{k}(2)$ so that steadystate measurements can be made. Recall that the outputs $y_{k}(1), y_{k}(2)$ of the vessels are measured in volts $(V)$ and that the control valve inputs $u_{k}(1), u_{k}(2)$ are also measured in $V$. With MARTS sampled at $h=0.1$ seconds, the steady-state tracking matrix $\Gamma$ was measured via the following procedure:

1. With the interconnecting valve between the water vessels set as $\theta=30^{\circ}$ and with the control valves set as half-opened, the by-pass and drainage valves were manually adjusted till the water level settled roughly at the chosen equilibrium heights in the water vessels.

2. A step input of magnitude $-1 V$ was applied to control valve 1 and the corresponding steady-state outputs were measured.

3. The water level was then restored back to the same equilibrium height chosen in step 1 , followed by a step input of magnitude $-1 V$ applied to control valve 2 .

4. $\Gamma$ was obtained experimentally to be:

$$
\Gamma=\left[\begin{array}{ll}
8.5 \times 10^{-2} & 1.0 \times 10^{-2} \\
1.0 \times 10^{-2} & 7.5 \times 10^{-2}
\end{array}\right]
$$

Open-loop responses of the system are given in Figures 8.5-1 and 8.5-2. 

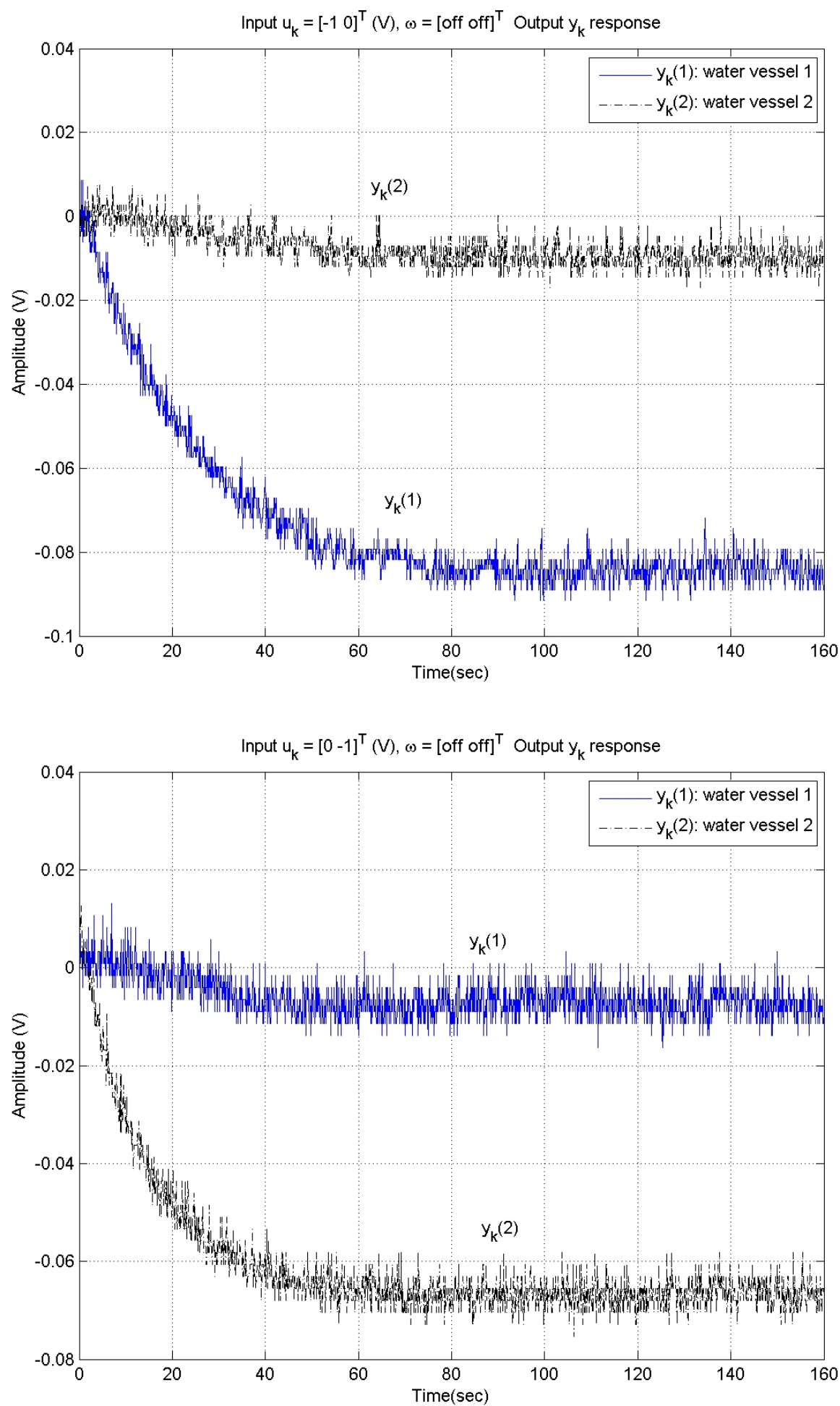

Figure 8.5-1: MARTS experiment, open-loop response. 

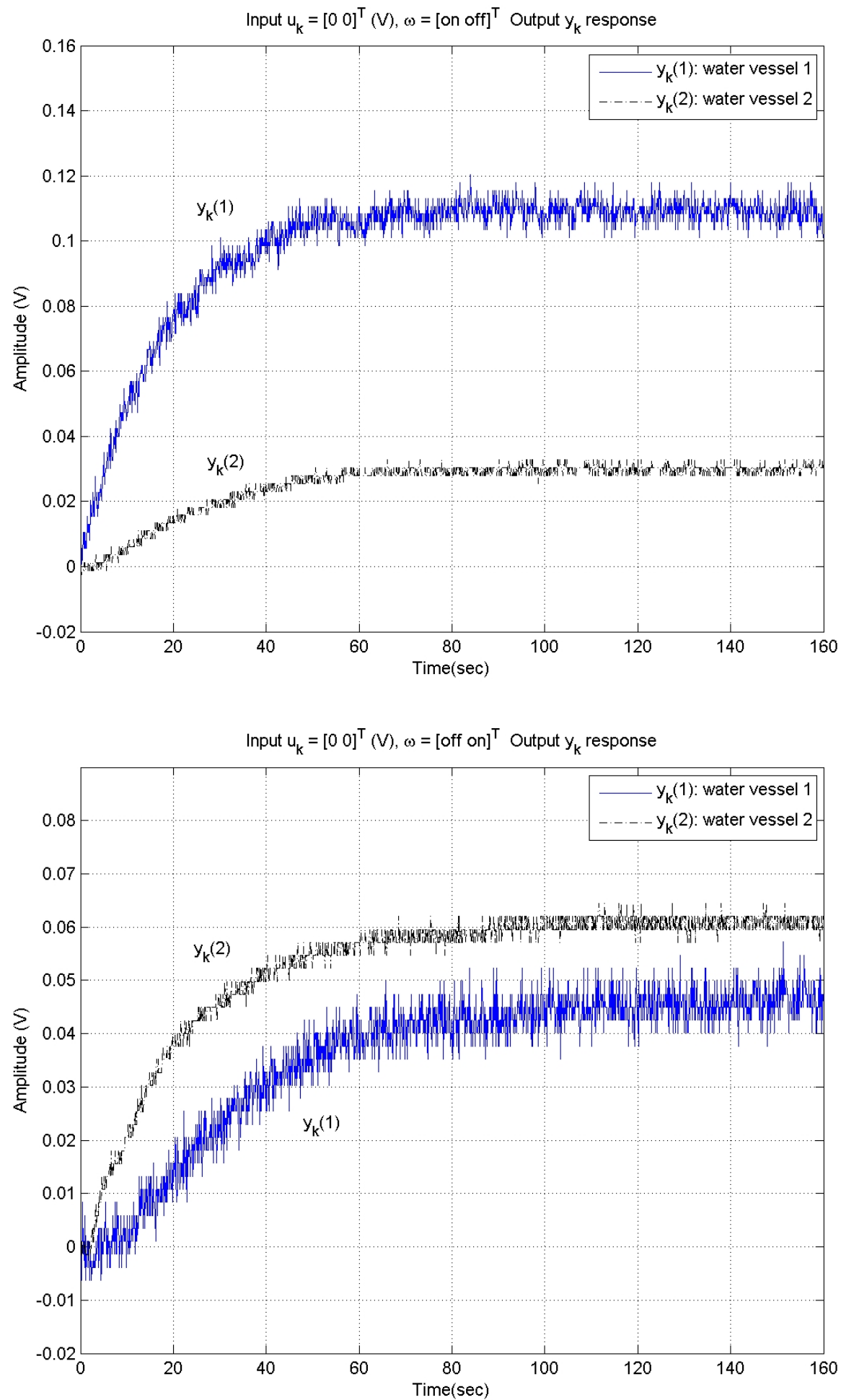

Figure 8.5-2: MARTS experiment, open-loop response. 


\subsection{Control Design for MARTS}

The two-term controller (2.6) is obtained by minimizing the performance index $J_{B y_{0}}$ (2.78) using $\theta=0, \mu=1$. The optimization is carried out under the system settings of:

$$
y_{0}=\left[\begin{array}{c}
-0.1 \\
-0.15
\end{array}\right], \quad \eta_{0}=\left[\begin{array}{l}
0 \\
0
\end{array}\right], \quad y_{\text {ref }}=\left[\begin{array}{l}
0 \\
0
\end{array}\right], \text { and } \quad \omega=\left[\begin{array}{l}
\text { off } \\
\text { off }
\end{array}\right] \text {. }
$$

The physical setup that corresponds to the above system settings is given as follows: On MARTS, with the interconnecting valve between the water vessels set as $\theta=30^{\circ}$ and with the control valves set as half-opened, the equilibrium water level was adjusted to be at $4.0 \mathrm{~V}$ for water vessel 1 and $4.2 \mathrm{~V}$ for vessel 2 by adjusting the by-pass and drainage valves manually. Control valve 1 was set at $0 \mathrm{~V}$ and control valve 2 at $1.26 \mathrm{~V}$ so that the valves were only half-opened when trying to achieve the desired equilibrium water level. Control valve 2 does not start at $0 \mathrm{~V}$ due to the linearization of the control valve characteristics. Before the start of each experiment, the water level was regulated to be at $3.9 \mathrm{~V}$ for vessel 1 and $4.05 \mathrm{~V}$ for vessel 2 to achieve the required setting of $y_{0}=\left[\begin{array}{ll}-0.1 & -0.15\end{array}\right]^{\top}$.

By carrying out the optimization process, the optimal control gains $K_{P}$ and $K_{I}$ are found to be

$$
K_{P}=6.4262 \times 10^{-1}, \quad K_{I}=7.7555 \times 10^{-2} .
$$

The measured closed-loop system waveforms are given in Figures 8.6-1 to 8.6-7. It is seen that the response of the system is fast and non-oscillatory. The controller gains and system responses obtained experimentally are very similar to those obtained in simulation (under the "Control Design 3 for MARTS" simulation example in Section 4.6). Furthermore, the peak output values apparent in Figures 8.6-6 and 8.6-7 are much smaller than the open-loop steady-state outputs for the input disturbances shown in Figure 8.5-2. It should be emphasized that at no time during the design phase, was system identification performed. The optimization process took a total of 10.5 hours to complete, and the results show that the proposed design approach works well on experimental systems. It is to be noted that the choice of carrying out the experiments by perturbing $y_{0}$ was made,

\footnotetext{
${ }^{1}$ The two-term controller (2.6) with a fixed $K_{P}$ and $K_{I}$ was used to ensure that the water level is at $3.9 \mathrm{~V}$ and $4.05 \mathrm{~V}$ before the start of each experiment. When an experiment begins, the controller gains are switched to the $K_{P}$ and $K_{I}$ values that we would like to compute a cost value for. The switch results in a discontinuous jump in the control input. Since $y_{0}$ is set to be relatively small in value, the jump in $u_{k}$ is also small in magnitude; thus, the amount of inrush water added over that small period of time is little and the effects on the calculation of the performance index is negligible compared to those from the measurement noise. Furthermore, this conclusion drawn was re-confirmed by turning on and off the pumps in rapid intermittent fashion with no change in the output waveform observed.
} 
to show that it is not essential to have actually apply physical tracking signals or disturbance signals to design a controller for a plant. A summary of the optimization results is given in Table 8.1, and the detailed optimization output can be found in Appendix C.4.

\begin{tabular}{ccccccc}
\hline $\begin{array}{c}\text { Initial } \\
K_{P}\end{array}$ & $\begin{array}{c}\text { Initial } \\
K_{I}\end{array}$ & $\begin{array}{c}\text { Final } \\
K_{P}\end{array}$ & $\begin{array}{c}\text { Final } \\
K_{I}\end{array}$ & $\begin{array}{c}\text { Initial } \\
\text { cost } J\end{array}$ & $\begin{array}{c}\text { Final } \\
\text { cost } J\end{array}$ & $\begin{array}{c}\text { Number of } \\
\text { function } \\
\text { evaluations }\end{array}$ \\
\hline $\begin{array}{c}1.00 \times \\
10^{-2}\end{array}$ & $5.00 \times$ & $6.43 \times$ & $7.76 \times$ & 1068.18 & 63.28 & 58 \\
\hline
\end{tabular}

Table 8.1: Summary of controller parameter optimization for the experimental MARTS system with $\theta=0$ and $\mu=1$. 

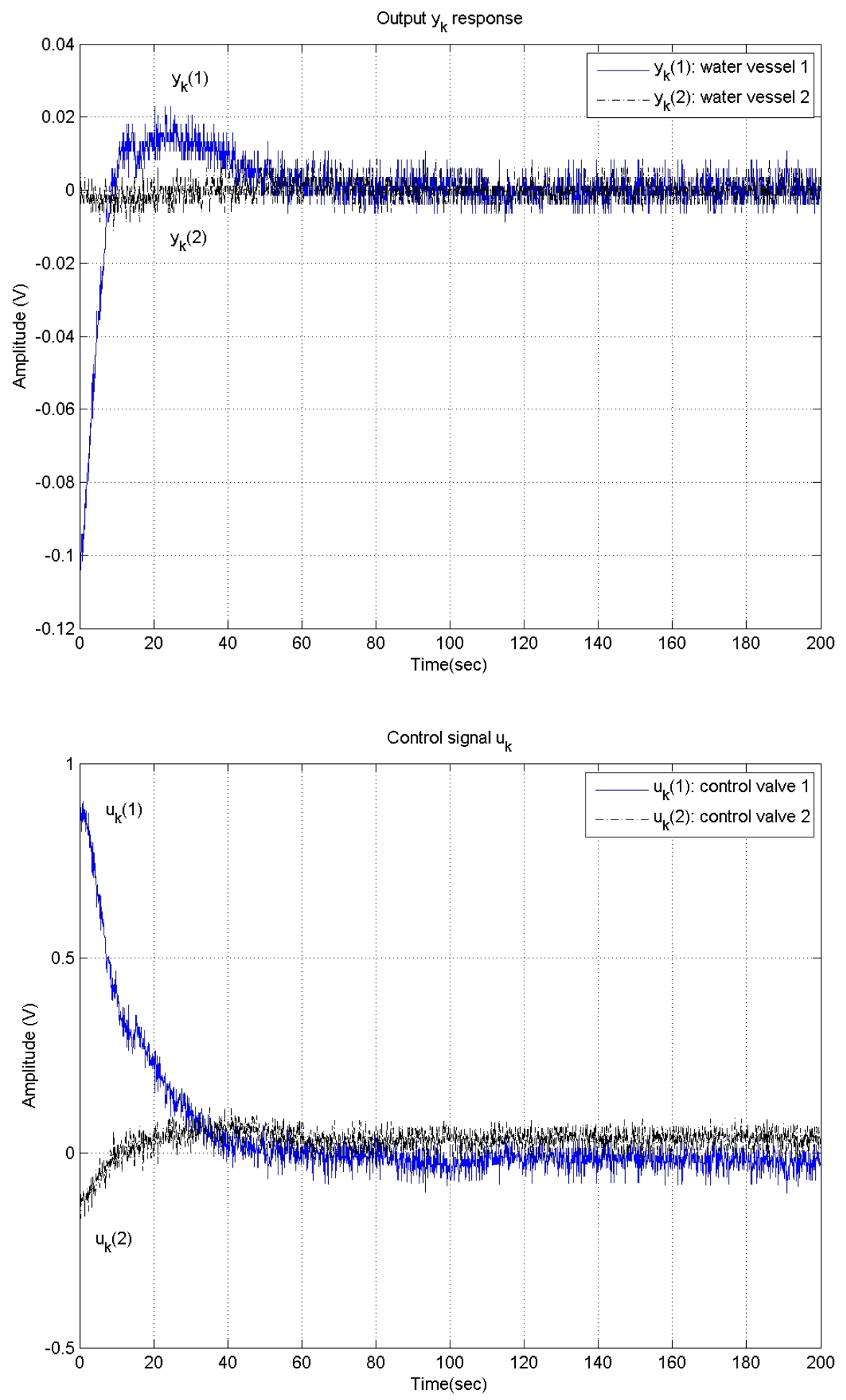

Figure 8.6-1: MARTS Experiment, Closed-Loop Response Two-Term Controller (2.6) with Controller Gains (8.1) Input $y_{0}=\left[\begin{array}{ll}-0.1 & 0\end{array}\right]^{\top}$. 

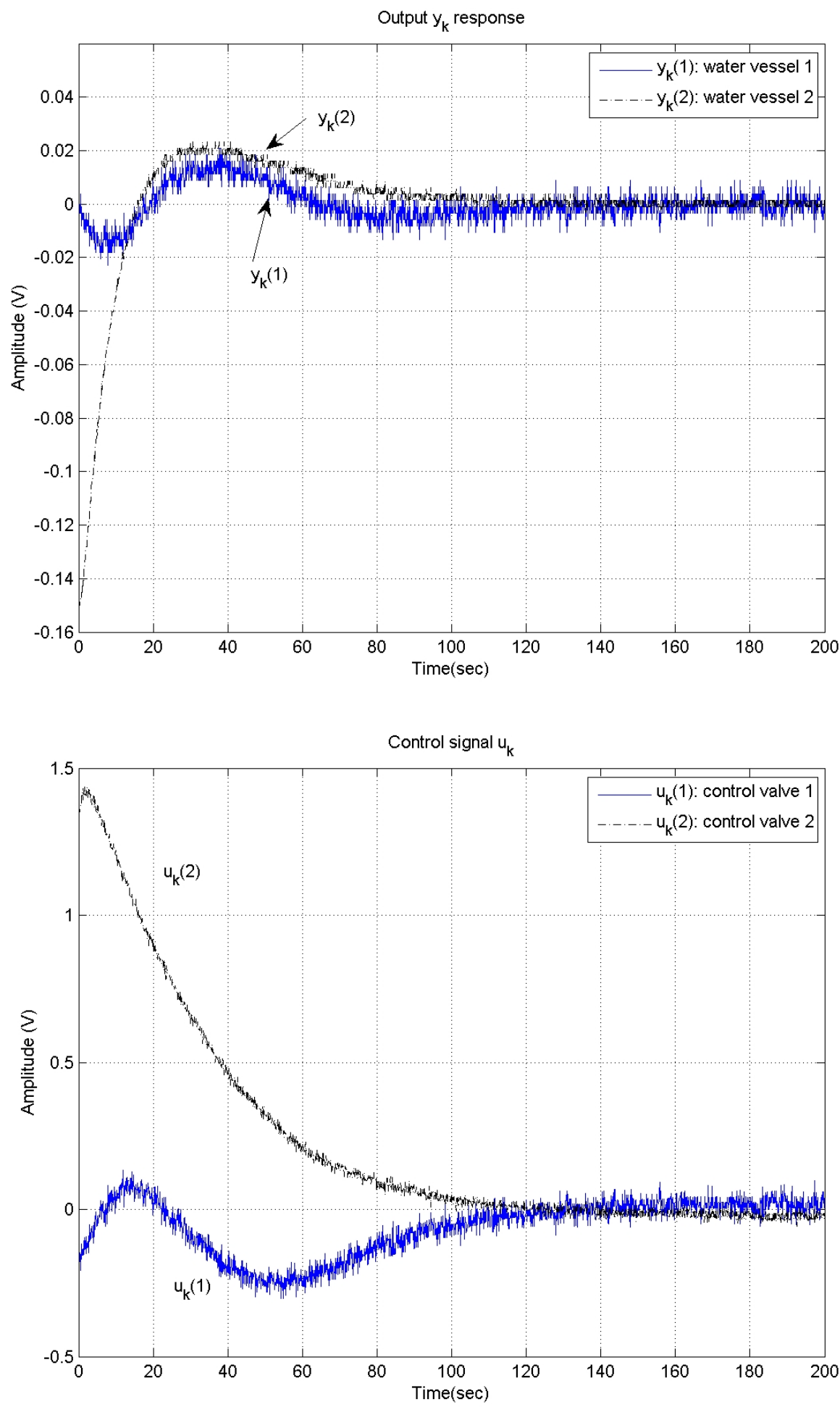

Figure 8.6-2: MARTS Experiment, Closed-Loop Response Two-Term Controller (2.6) with Controller Gains (8.1) Input $y_{0}=\left[\begin{array}{ll}0 & -0.15\end{array}\right]^{\top}$. 

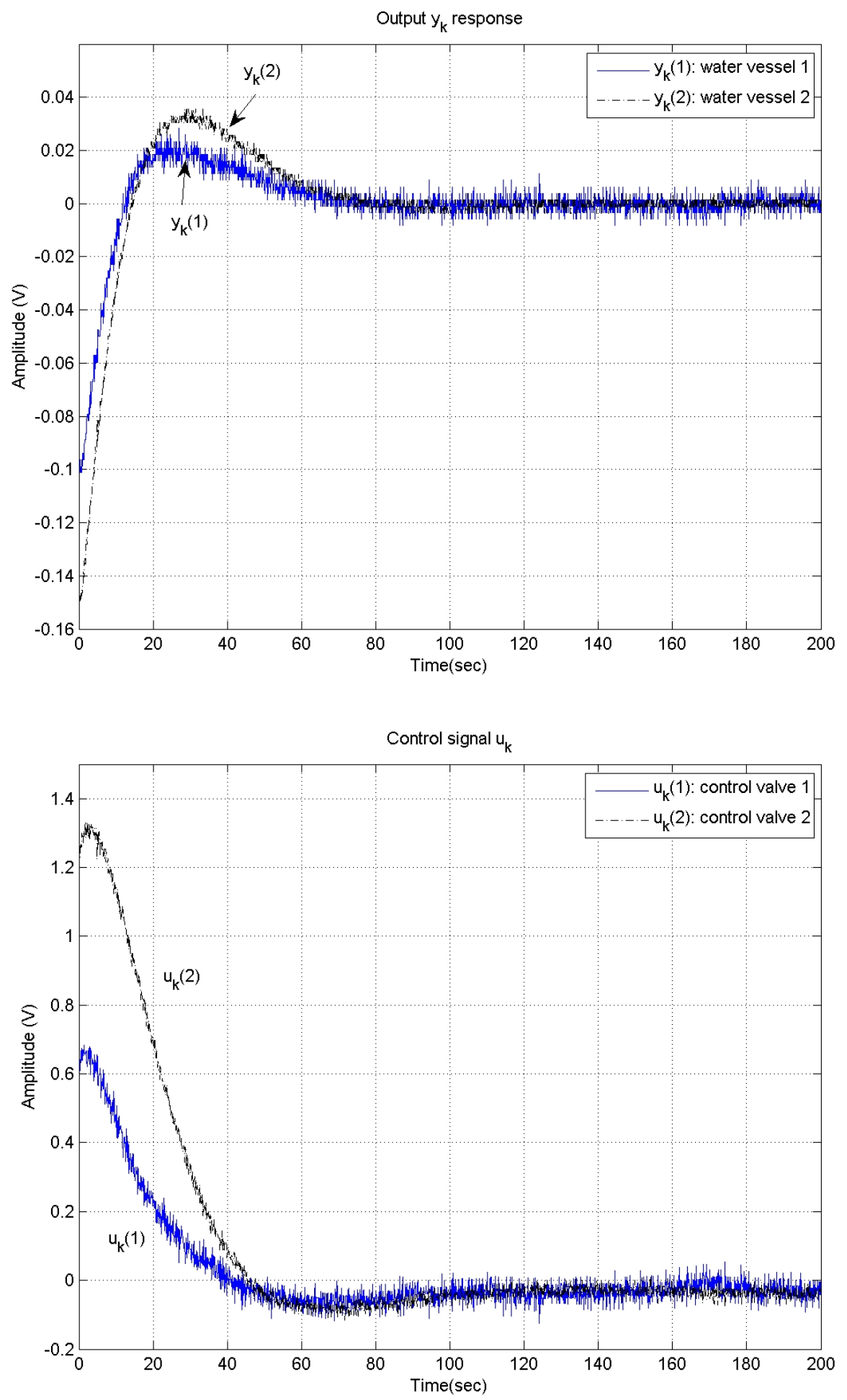

Figure 8.6-3: MARTS Experiment, Closed-Loop Response Two-Term Controller (2.6) with Controller Gains (8.1) Input $y_{0}=\left[\begin{array}{ll}-0.1 & -0.15\end{array}\right]^{\top}$. 

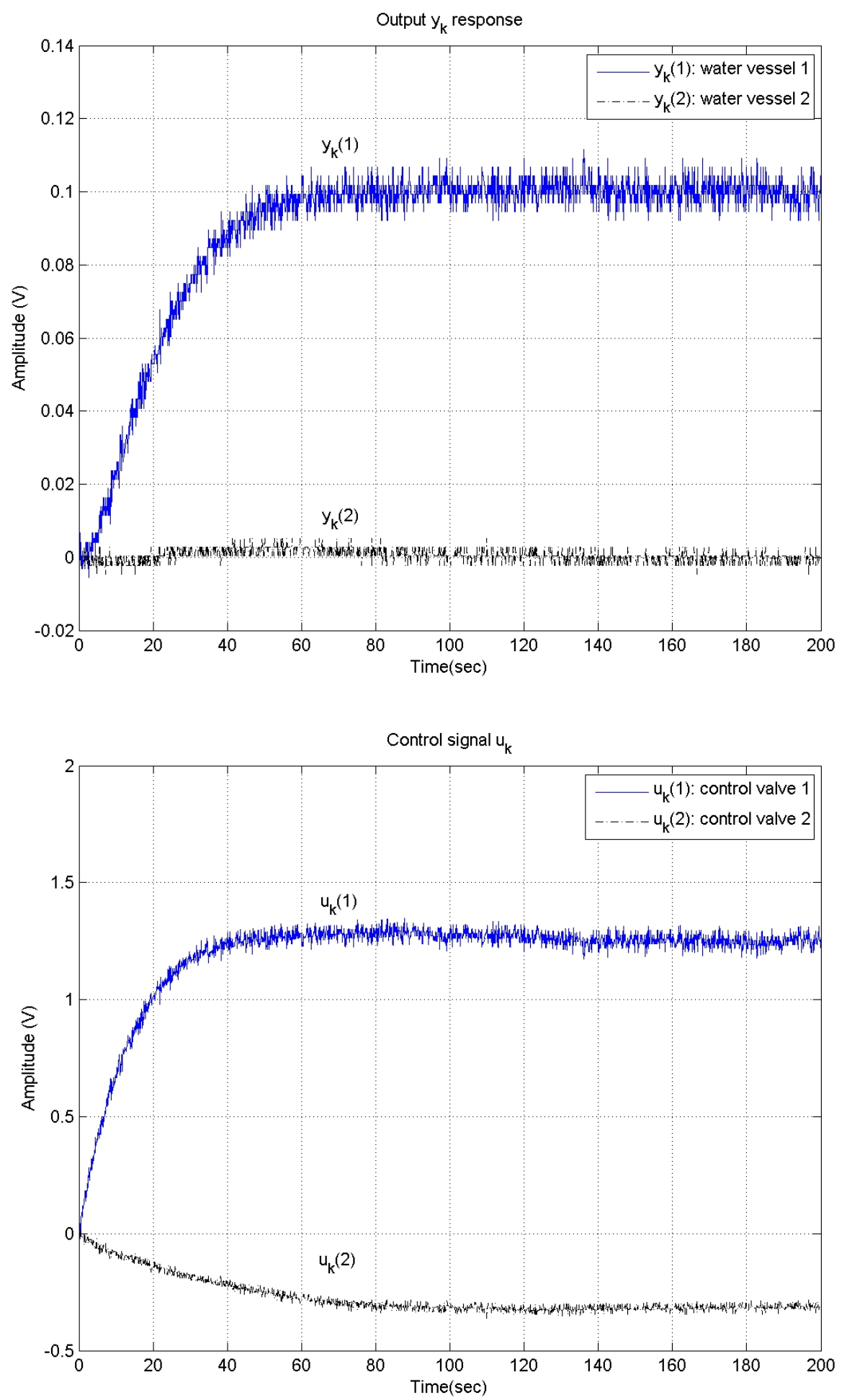

Figure 8.6-4: MARTS Experiment, Closed-Loop Response Two-Term Controller (2.6) with Controller Gains (8.1) Input $y_{\text {ref }}=\left[\begin{array}{ll}0.1 & 0\end{array}\right]^{\top}$. 

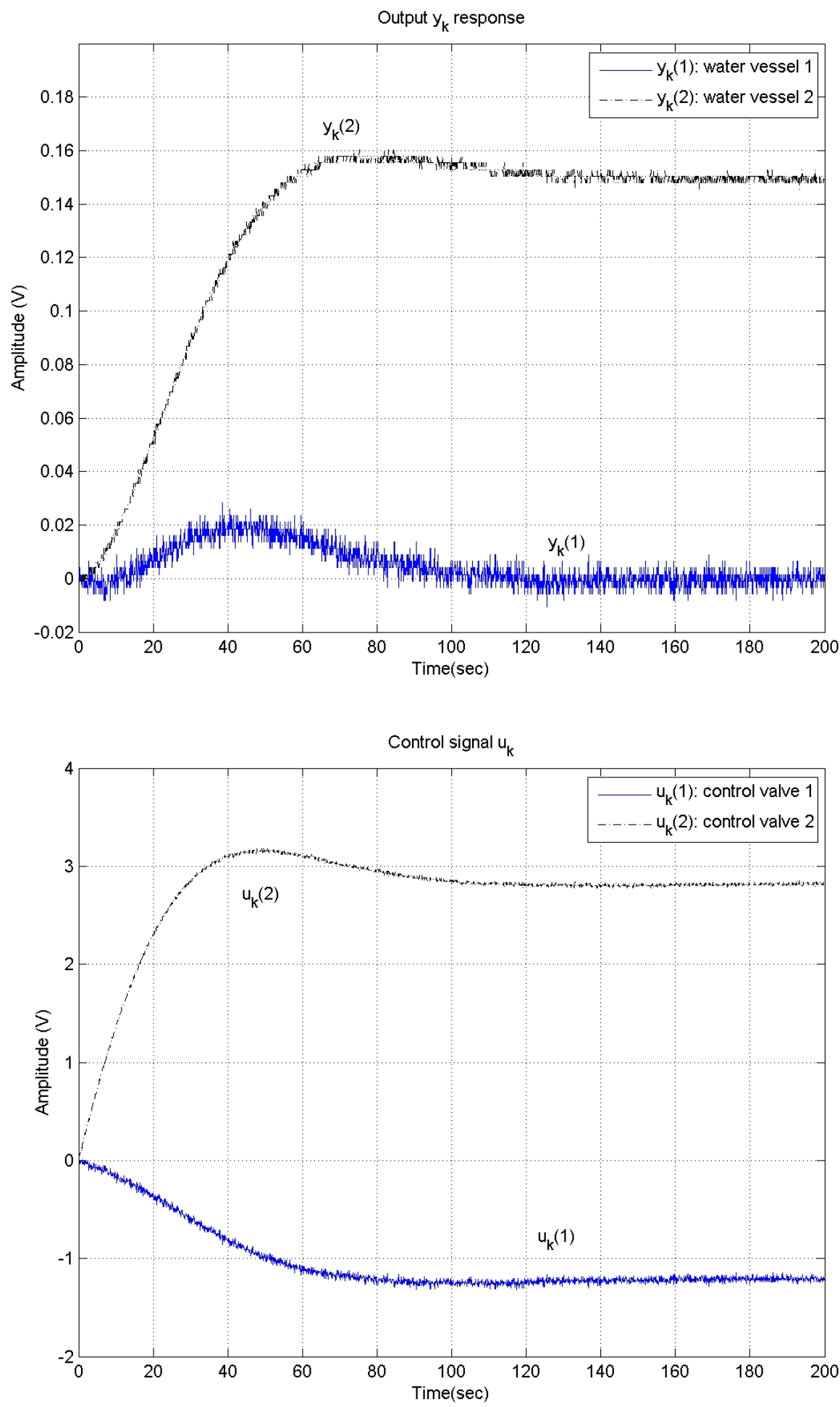

Figure 8.6-5: MARTS Experiment, Closed-Loop Response Two-Term Controller (2.6) with Controller Gains (8.1) Input $y_{r e f}=\left[\begin{array}{ll}0 & 0.15\end{array}\right]^{\top}$. 

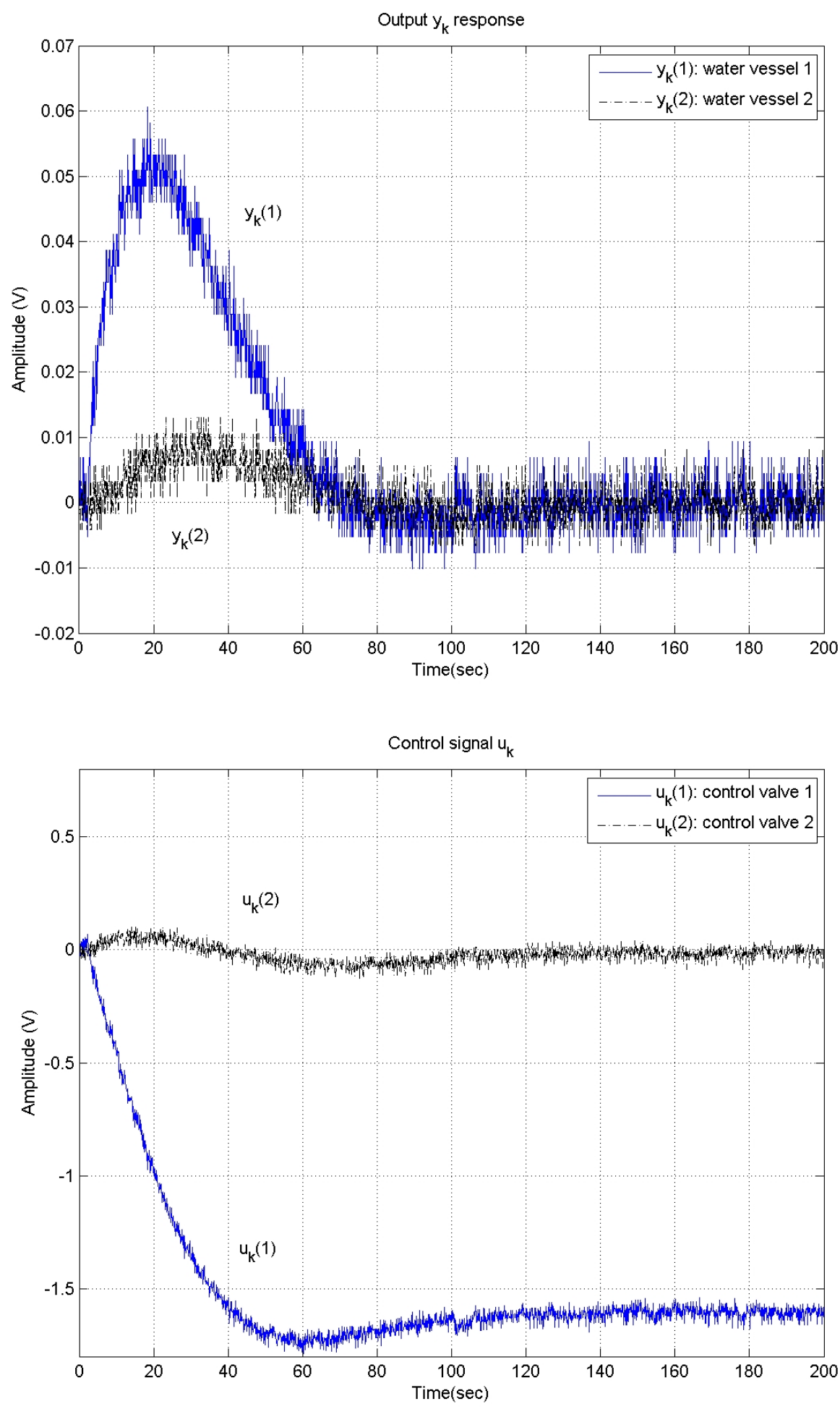

Figure 8.6-6: MARTS Experiment, Closed-Loop Response Two-Term Controller (2.6) with Controller Gains (8.1) Input $\omega(1)=$ on, and $\omega(2)=$ off . 

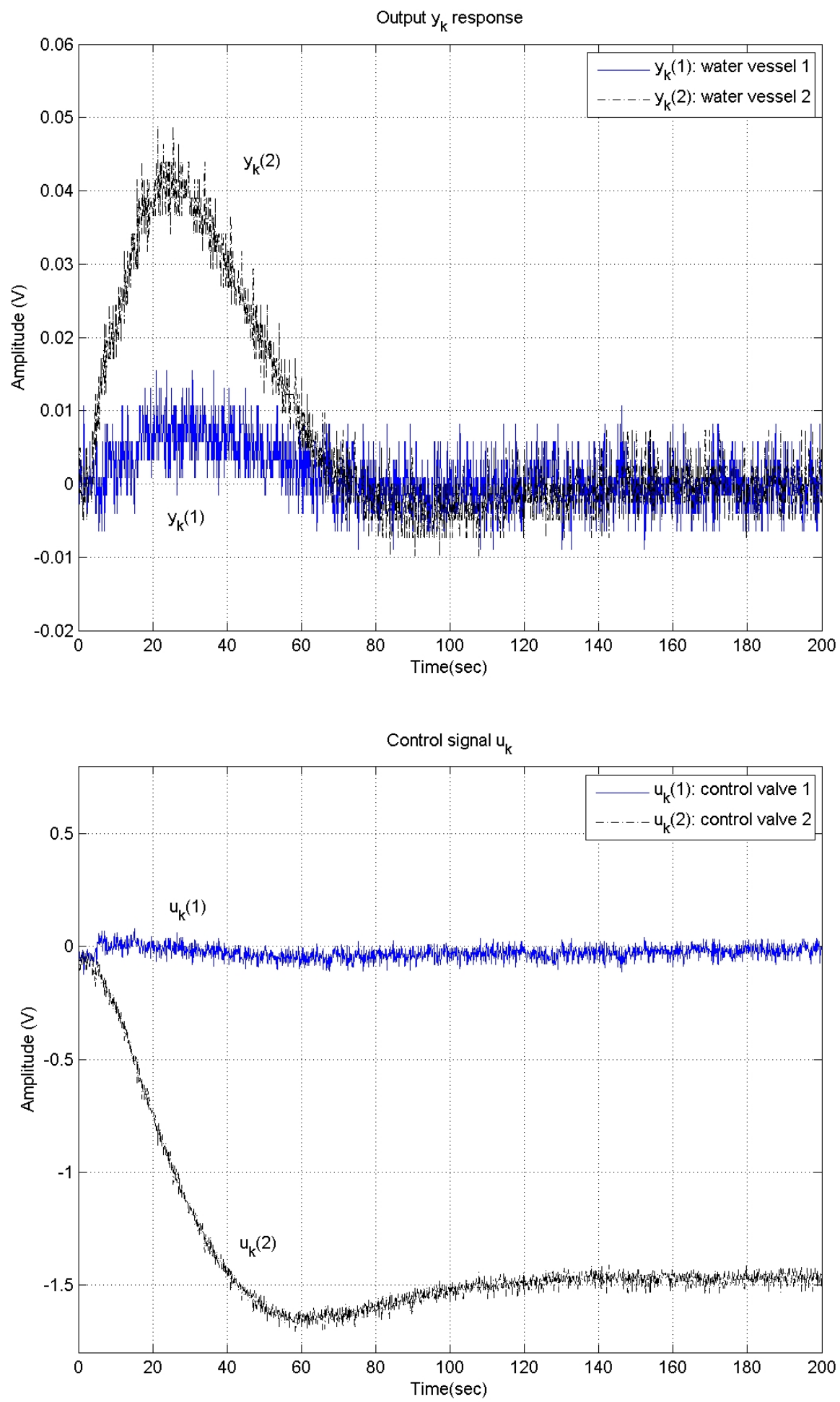

Figure 8.6-7: MARTS Experiment, Closed-Loop Response Two-Term Controller (2.6) with Controller Gains (8.1) Input $\omega(1)=$ off, and $\omega(2)=$ on . 


\section{Chapter 9}

\section{Conclusion}

In this chapter, the main results in this thesis are summarized, and possible future work is discussed.

\subsection{Summary of Results}

In this thesis, a new method of designing multivariable robust servomechanism controllers for MIMO open-loop stable systems, subject to constant references and disturbances, has been proposed and successfully applied to a real physical plant. The method requires no knowledge of the plant model nor of the order of the system, but assumes the inputs can be excited, and the outputs can be measured. The design process is carried out within a closed-loop setting. The controller parameters are optimized by minimizing the average cost of a performance index over the plant initial conditions, or disturbance/ tracking signals uniformly distributed on a unit disk ball. The performance indices are expressed in a form where the cost can be directly computed via measured data. Once the cost value is computed, it is used as a single data point in the controller parameter optimization and an algorithm such as the Nelder-Mead algorithm is then employed to find the local minimizer. An important advantage that the method offers is that optimizing for tracking performance can be achieved by perturbing only the initial conditions of the servocompensator while minimizing the noise insensitive performance index. The method is well suited for applications that exhibit actuator saturation, and the order of the resulting controller obtained is low and is independent of the system order. An experimental application study of the proposed controller design approach was then performed on a multivariable hydraulic system (MARTS), which had industrial 
actuators and sensors, and the results obtained indicate that the design method is feasible to use in an industrial environment.

\section{$9.2 \quad$ Future Work}

A natural extension of this work is to consider ways of applying more general controller structures that are optimal in some sense for different classes of systems or if more a priori knowledge of the plant is known. For example, one can consider three-term controllers or state based controllers to replace the PI controllers used in the thesis. Once the controller structure is determined, one can proceed to find the optimal controller parameters using the same method presented in this thesis. Finally, it would be of interest to extend the theory and application of the proposed method to the design of decentralized controllers. 


\section{References}

[1] Y. Zhu, Multivariable system identification for process control, $1^{\text {st }}$ ed. Pergamon, Amsterdam, New York; 2001.

[2] J. G. Ziegler and N. B. Nichols, "Optimum settings for automatic controllers," Trans. ASME, vol. 64, 1942, pp. 759-768.

[3] K. J. Åström and B. Wittenmark, Computer controlled systems: Theory and design, $3^{\text {rd }}$ ed. Prentice-Hall, Upper Saddle River, New Jersey; 1997.

[4] B. W. Bequette, Process control: Modeling, design and simulation, Prentice-Hall, Upper Saddle River, New Jersey; 2003.

[5] K. J. Åström and T. Hägglund, PID controllers: Theory, design and tuning, $2^{\text {nd }}$ ed. Instrument Society of America, Research Triangle Park, North Carolina; 1995.

[6] K. J. Åström and T. Hägglund, "Automatic tuning of simple regulators with specifications on phase and amplitude margins," Automatica, vol. 20, no. 5, 1984, pp. 645651.

[7] K. J. Åström, T. Hägglund, C. C. Hang and W. K. Ho, "Automatic tuning and adaptation for PID controllers - a survey," Control Eng. Practice, vol. 1, No. 4, 1993, pp. 699-714.

[8] E. H. Bristol, "On a new measure of interaction for multivariable process control," IEEE Trans. on Auto. Control, vol. 11, 1966, pp. 133-134.

[9] K. E. Häggblom, "Limitations and use of the RGA as a controllability measure," Proc. Finnish Automation Days, Helsinki, Finland, 1995, pp. 178-183.

[10] K. B. Ariyur and M. Krstić, Real-time optimization by extremum seeking control, Wiley-Interscience, Hoboken, New Jersey; 2003. 
[11] M. Krstić and H. Wang, "Stability of extremum seeking feedback for general nonlinear dynmaic systems," Automatica, vol. 36, 2000, pp. 595-601.

[12] Nusawardhana and S. H. Żak, "Extremum seeking using analog nonderivative optimizers," Proc. of the American Control Conf., Colorado, USA, June 4-6, 2003, pp. 3242-3247.

[13] N. J. Killingsworth and M. Krstić, "PID tuning using extremum seeking: Online, model-free performance optimization," IEEE Control Syst. Mag., vol. 26, no. 1, Feb. 2006, pp. 70-79.

[14] Y. Tan, D. Nešić and I. Mareels, "On non-local stability properties of extremum seeking control," Automatica, vol. 42, 2006, pp. 889-903.

[15] M. Krstić, "Performance improvement and limitations in extremum seeking control," Systems 8 Control Letters, vol. 39, 2000, pp. 313-326.

[16] P. Li, Y. Li, and J. E. Seem, "Efficient operation of air-side economizer using extremum seeking control," ASME Trans. - J. of Dynamic Systems, Measurement and Control, vol. 132, no. 3, 2010, pp. 031009 (10 pages).

[17] H. Hjalmarsson, M. Gevers, S. Gunnarsson, and O. Lequin, "Iterative feedback tuning: Theory and applications," IEEE Control Syst. Mag., vol. 18, no. 4, 1998, pp. 26-41.

[18] H. Hjalmarsson and T. Birkeland, "Iterative feedback tuning of linear time-invariant MIMO systems," Proc. of the $37^{\text {th }}$ IEEE Conf. on Decision and Control, Tampa, Florida, Dec. 1998, pp. 3893-3898.

[19] A. S. Foss, "Critique of chemical process control theory," IEEE Trans. Auto. Control, vol. AC-18, 1973, pp. 646-652.

[20] L. B. Jemaa and E. J. Davison, "Performance limitations in the robust servomechanism problem for discrete-time LTI systems," IEEE Trans. on Auto. Control, vol. 48, no. 8, Aug. 2003, pp. 1299-1311.

[21] E. J. Davison, "Multivariable tuning regulators: The feedforward and robust control of a general servomechanism problem," IEEE Trans. on Auto. Control, vol. AC-21, Feb. 1976, pp. 35-47. 
[22] E. J. Davison and B. M. Scherzinger, "Perfect control of the robust servomechanism problem," IEEE Trans. on Auto. Control, vol. AC-32, no. 8, Aug. 1987, pp. 689-702.

[23] E. J. Davison, "CAD of controllers for the servomechanism problem subject to control signal saturation constraints," $13^{\text {th }}$ IFAC World Congress, San Francisco, CA, July 1996, vol. D, paper 29-166, pp. 61-67.

[24] J. Jiao, "Multivariable controller design for systems with time delays," master's thesis, University of Toronto, Department of Electrical and Computer Engineering, 1988.

[25] E. J. Davison and I. Ferguson, "The design of controllers for the multivariable robust servomechanism problems using parameter optimization methods," IEEE Trans. on Auto. Control, vol. AC-26, no. 1, February 1981, pp. 93-110.

[26] B. Roszak and E. J. Davison, "The servomechanism problem for unknown MIMO LTI positive systems: Feedforward and robust tuning regulators," 2008 American Control Conf., Washington, USA, June 11-13, 2008, pp. 4821-4826.

[27] H. K. Khalil, "Singular perturbations," in Nonlinear Systems, $3^{\text {rd }}$ ed. Prentice Hall, Upper Saddle River, New Jersey; 2002, ch. 11, pp. 423-459.

[28] B. Roszak and E. J. Davison, "Tuning regulators for tracking MIMO positive LTI systems," Systems Control Report No. 0701, University of Toronto, Department of Electrical and Computer Engineering, Jan. 10, 2007.

[29] D. E. Davison and E. J. Davison, "Optimal transient response shaping for the discrete-time servomechanism problem," 2003 European Control Conf. (ECC2003), Cambridge, UK, Sept. 2003, presented 6 pages in Technical Session 8, Sept. 8.

[30] D. E. Davison and E. J. Davison, "Optimal transient response shaping of the servomechanism problem," J. of Optim. Theory and Appl., vol. 115, no. 3, December 2002, pp. 491-515.

[31] E. J. Davison, D. E. Davison, and S. Lam, "Multivariable three-term optimal controller design for large-scale systems," 2009 IEEE Control and Decision Conf., Shanghai, P. R. China, Dec. 16-18, 2009, pp. 940-945. 
[32] M. Loéve, "Probability theory I," Graduate Texts in Mathematics, vol. 45, $4^{\text {th }}$ ed. Springer-Verlag, 1977, pp. 12.

[33] J. A. Nelder and R. Mead, "A simplex method for function minimization," The Comp. J., vol. 7 issue 4, 1965, pp. 308-313.

[34] J. H. Mathews and K. K. Fink, "Numerical optimization," in Numerical Methods Using Matlab, $4^{\text {th }}$ ed. Prentice Hall, Upper Saddle River, New Jersey; 2004.

[35] J. C. Lagarias, J. A. Reeds, M. H. Wright, and P. E. Wright, "Convergence properties of the Nelder-Mead simplex method in low dimensions," SIAM J. Optim., vol. 9, no. 1, 1998, pp. 112-147.

[36] T. G. Kolda, R. M. Lewis, and V. Torczon, "Optimization by direct search: New perspectives on some classical and modern methods," SIAM Review, vol. 45, no. 3, 2003, pp. 385-482.

[37] S. Singer and S. Singer, "Efficient implementation of the Nelder-Mead search algorithm ," Appl. Num. Anal. Comp. Math, vol. 1, no. 2, Dec. 2004, pp. 524-534.

[38] D. Whitley, "An overview of evolutionary algorithms: Practical issues and common pitfalls," Info. and Software Tech., vol. 43, 2001, pp. 817-831.

[39] M. Chang and E. J. Davison, "Adaptive control applied to unmodelled multivariable systems," Systems Control Report 9306, University of Toronto, Department of Electrical and Computer Engineering, June 1993.

[40] T. J. Williams and R. E. Otto, "A generalized chemical model for the investigation of computer control," AIEE Trans. Part I (Comm. \& Electronics), vol. 79, Nov. 1960, pp. $458-473$.

[41] D. J. N. Limebeer, R. G. Harley and S. M. Schuck, "Subsynchronous resonance of the Koeberg turbogenerators and of a laboratory micro-alternator system," Trans. S. Afr. Inst. Electr. Eng., vol. 70, 1979, pp. 278-297.

[42] E. J. Davison and H. Fathi, "Description of control laboratory multivariable experiment: 'MARTS'," Proc. of the $38^{\text {th }}$ Conf. on Decision and Control, Phoenix, USA, 1999, pp. 813-818. 
[43] B. Roszak and E. J. Davison, "Tuning clamping regulators for positive SISO unknown LTI systems: Industrial hydraulic systems results - Waterworks experimental results," 2009 American Control Conf., St. Louis, USA, June 2009, pp. 3089-3094. 


\section{Appendix A}

\section{Mathematical Derivations}

This appendix contains the derivation of some equations that were used in the chapters of the thesis document.

\section{A.1 Equivalent Steady-State Control Input Expres- sions}

The relationship of $\left(C\left(I-\left(A-B S K_{P} C\right)\right)^{-1} B\right)^{-1}=\left(C(I-A)^{-1} B\right)^{-1}+S K_{P}$ in Section 2.2.1 can be derived as follows. Consider the system

$$
\begin{aligned}
x_{k+1} & =A x_{k}+B u_{k} \\
y_{k} & =C x_{k} \\
u_{k} & =K y_{k}+\hat{u}_{k}
\end{aligned}
$$

where $K=-S K_{P}$. There are two approaches in determining the steady-state relationship between $\hat{u}_{k}$ and $y_{k}$ :

Approach 1

$$
\begin{aligned}
x_{\infty} & =A x_{\infty}+B K C x_{\infty}+B \hat{u}_{\infty} \\
& =(A+B K C) x_{\infty}+B \hat{u}_{\infty} \\
\Rightarrow \quad x_{\infty} & =\left(I-\left(A-B S K_{P} C\right)\right)^{-1} B \hat{u}_{\infty} \\
y_{\infty} & =C\left(I-\left(A-B S K_{P} C\right)\right)^{-1} B \hat{u}_{\infty} \\
\hat{u}_{\infty} & =\left(C\left(I-\left(A-B S K_{P} C\right)\right)^{-1} B\right)^{-1} y_{\infty} .
\end{aligned}
$$


Approach 2

$$
\begin{aligned}
x_{\infty} & =A x_{\infty}+B u_{\infty} \\
\Rightarrow \quad x_{\infty} & =(I-A)^{-1} B u_{\infty} \\
& =(I-A)^{-1} B\left(K y_{\infty}+\hat{u}_{\infty}\right) \\
& =-(I-A)^{-1} B S K_{P} y_{\infty}+(I-A)^{-1} B \hat{u}_{\infty} \\
y_{\infty} & =-C(I-A)^{-1} B S K_{P} y_{\infty}+C(I-A)^{-1} B \hat{u}_{\infty} \\
\hat{u}_{\infty} & =\left(C(I-A)^{-1} B\right)^{-1}\left(I+C(I-A)^{-1} B S K_{P}\right) y_{\infty} \\
& =\left(\left(C(I-A)^{-1} B\right)^{-1}+S K_{P}\right) y_{\infty} .
\end{aligned}
$$

Therefore, $\left(C(I-(A-B K C))^{-1} B\right)^{-1}=\left(C(I-A)^{-1} B\right)^{-1}+S K_{P}$.

\section{A.2 Initial Conditions for Optimization Problem B}

The expression for $\left[\begin{array}{ll}\Delta x_{0} & \Delta \eta_{0}\end{array}\right]^{\top}=\left[\begin{array}{ll}x_{0}-x_{\infty} & \eta_{0}-\eta_{\infty}\end{array}\right]^{\top}$ given in Section 2.4 .2 can be derived as follows. On applying the controller (2.6) to the plant (2.1) we obtain the following closed-loop augmented system:

$$
\left[\begin{array}{l}
x_{k+1} \\
\eta_{k+1}
\end{array}\right]=\left[\begin{array}{cc}
A-B S K_{P} C & -B S K_{I} \\
h C & I
\end{array}\right]\left[\begin{array}{l}
x_{k} \\
\eta_{k}
\end{array}\right]+\left[\begin{array}{cc}
E-B S K_{P} F & 0 \\
h F & -h
\end{array}\right]\left[\begin{array}{c}
\omega \\
y_{r e f}
\end{array}\right] .
$$

At steady state, the system becomes

$$
\begin{aligned}
{\left[\begin{array}{l}
x_{\infty} \\
\eta_{\infty}
\end{array}\right] } & =\left[\begin{array}{cc}
A-B S K_{P} C & -B S K_{I} \\
h C & I
\end{array}\right]\left[\begin{array}{l}
x_{\infty} \\
\eta_{\infty}
\end{array}\right]+\left[\begin{array}{cc}
E-B S K_{P} F & 0 \\
h F & -h
\end{array}\right]\left[\begin{array}{c}
\omega \\
y_{r e f}
\end{array}\right] \\
\Rightarrow \quad\left[\begin{array}{l}
x_{\infty} \\
\eta_{\infty}
\end{array}\right] & =\left[\begin{array}{cc}
I-\left(A-B S K_{P} C\right) & B S K_{I} \\
-h C & 0
\end{array}\right]\left[\begin{array}{cc}
E-B S K_{P} F & 0 \\
h F & -h
\end{array}\right]\left[\begin{array}{c}
\omega \\
y_{r e f}
\end{array}\right] .
\end{aligned}
$$

Therefore, we obtain

$$
\left[\begin{array}{c}
\Delta x_{0} \\
\Delta \eta_{0}
\end{array}\right]=\left[\begin{array}{l}
x_{0} \\
\eta_{0}
\end{array}\right]-\left[\begin{array}{cc}
I-\left(A-B S K_{P} C\right) & B S K_{I} \\
-h C & 0
\end{array}\right]^{-1}\left[\begin{array}{cc}
E-B S K_{P} F & 0 \\
h F & -h
\end{array}\right]\left[\begin{array}{c}
\omega \\
y_{r e f}
\end{array}\right] .
$$




\section{Appendix B}

\section{Model of Williams and Otto Chemical Plant}

The following continuous state-space model is used in the "Williams and Otto Chemical Plant Example" in Chapter 5. In the example, the discrete model used was generated by sampling the continuous-time model with a sampling period $h=0.01$ seconds.

$$
\begin{aligned}
\dot{x}(t) & =\mathcal{A} x(t)+\mathcal{B} u(t)+\mathcal{E} \omega \\
y(t) & =\mathcal{C} x(t)+\mathcal{D} u(t)+\mathcal{F} \omega
\end{aligned}
$$

\begin{tabular}{|c|c|c|c|c|}
\hline \multicolumn{5}{|c|}{ Columns 1 through 5} \\
\hline$-4.0740 e+001$ & $-6.3600 e+000$ & 0 & 0 & 0 \\
\hline$-2.0100 e+001$ & $-3.1470 e+001$ & $-6.8400 e+001$ & 0 & 0 \\
\hline $4.0200 e+001$ & $3.7800 e+000$ & $-1.8244 e+002$ & 0 & $-7.3500 e+000$ \\
\hline 0 & $8.9400 e+000$ & $1.3680 e+002$ & $-2.0640 e+001$ & 0 \\
\hline 0 & $4.4700 e+000$ & $5.5900 e+001$ & 0 & $-2.4315 e+001$ \\
\hline 0 & 0 & $3.7500 e+001$ & 0 & $1.1025 e+001$ \\
\hline $3.0300 e+003$ & $1.4495 e+003$ & $8.1680 e+003$ & 0 & $1.9060 e+002$ \\
\hline 0 & 0 & 0 & 0 & 0 \\
\hline 0 & 0 & 0 & 0 & 0 \\
\hline $3.5000 e+002$ & 0 & 0 & 0 & 0 \\
\hline 0 & $3.5000 e+002$ & 0 & 0 & 0 \\
\hline 0 & 0 & $3.5000 e+002$ & 0 & 0 \\
\hline 0 & 0 & 0 & $3.5000 e+002$ & 0 \\
\hline 0 & 0 & 0 & 0 & $3.5000 e+002$ \\
\hline 0 & 0 & 0 & 0 & 0 \\
\hline
\end{tabular}

where 


$\begin{array}{lllll}0 & 0 & 0 & 0 & 0 \\ 0 & 0 & 0 & 0 & 0 \\ 0 & 0 & 0 & 0 & 0 \\ 0 & 0 & 0 & 0 & 0 \\ 0 & 0 & 0 & 0 \\ 0 & 0 & 0 & 0 & 0 \\ 0 & 0 & 0 & 0 & 0 \\ 0 & 0 & 0 & 0 & 0 \\ 0 & 0 & 0 & 0 & 0 \\ 0 & 0 & 0 & 0 & 0 \\ 0 & 0 & 0 & 0 & 0 \\ 0 & 0 & 0 & 0 & 0 \\ 0 & 0 & 0 & 0 & 0 \\ 0 & 0 & 0 & 0 & 0 \\ 0 & 0 & 0 & 0 & 0 \\ 0 & 0 & 0 & 0 & 0 \\ 0 & 0 & 0 & 0 & 0 \\ 0 & 0 & 0 & 0 & 0 \\ 0 & 0 & 0 & 0 & 0 \\ 0 & 0 & 0 & 0 & 0 \\ 0 & 0 & 0 & 0 & 0 \\ 0 & 0 & 0 & 0 & 0 \\ 0 & 0 & 0 & 0 & 0 \\ 0 & 0 & 0 & 0 & 0 \\ 0 & 0 & 0 & 0 & 0 \\ 0 & 0 & 0 & 0 & 0\end{array}$

Columns 6 through 10

$\begin{array}{rrrrr}0 & 0 & 0 & 0 & 0 \\ 0 & 0 & 0 & 0 & 0 \\ 0 & 0 & 0 & 0 & 0 \\ 0 & 0 & 0 & 0 & 0 \\ 0 & 0 & 0 & 0 & 0 \\ -2.0640 e+001 & 0 & 0 & 0 & 0 \\ 0 & -3.5820 e+001 & 0 & 0 & 0 \\ 0 & 4.3600 e+002 & -6.0300 e+002 & 1.6700 e+002 & 0 \\ 0 & 0 & 1.7700 e+001 & -2.0269 e+002 & 0 \\ 0 & 0 & 0 & 0 & -3.5000 e+002 \\ 0 & 0 & 0 & 0 & 0 \\ 0 & 0 & 0 & 0 & 0 \\ 0 & 0 & 0 & 0 & 0 \\ 0 & 0 & 0 & 0 & 0 \\ 3.5000 e+00 & 0 & 0 & 0 & 0 \\ 0 & 0 & 0 & 0 & 0 \\ 0 & 0 & 0 & 0 & 0 \\ 0 & 0 & 0 & 0 & 0 \\ 0 & 0 & 0 & 0 & 0 \\ 0 & 0 & 0 & 0 & 0 \\ 0 & 0 & 0 & 0 & 0 \\ 0 & 0 & 0 & 0 & 0 \\ 0 & 0 & 0 & 0 & 0\end{array}$




$\begin{array}{lllll}0 & 0 & 0 & 0 & 0 \\ 0 & 0 & 0 & 0 & 0 \\ 0 & 0 & 0 & 0 & 0 \\ 0 & 0 & 0 & 0 & 0 \\ 0 & 0 & 0 & 0 & 0 \\ 0 & 0 & 0 & 0 & 0 \\ 0 & 0 & 0 & 0 & 0 \\ 0 & 0 & 0 & 0 & 0 \\ 0 & 0 & 0 & 0 & 0 \\ 0 & 0 & 0 & 0 & 0 \\ 0 & 0 & 0 & 0 & 0 \\ 0 & 0 & 0 & 0 & 0 \\ 0 & 0 & 0 & 0 & 0 \\ 0 & 0 & 0 & 0 & 0 \\ 0 & 0 & 0 & 0 & 0 \\ 0 & 0 & 0 & 0 & 0\end{array}$

Columns 11 through 15

\begin{tabular}{|c|c|c|c|c|}
\hline 0 & 0 & 0 & 0 & 0 \\
\hline 0 & 0 & 0 & 0 & 0 \\
\hline 0 & 0 & 0 & 0 & 0 \\
\hline 0 & 0 & 0 & 0 & 0 \\
\hline 0 & 0 & 0 & 0 & 0 \\
\hline 0 & 0 & 0 & 0 & 0 \\
\hline 0 & 0 & 0 & 0 & 0 \\
\hline 0 & 0 & 0 & 0 & 0 \\
\hline 0 & 0 & 0 & 0 & 0 \\
\hline 0 & 0 & 0 & 0 & 0 \\
\hline$-3.5000 e+002$ & 0 & 0 & 0 & 0 \\
\hline 0 & $-3.5000 e+002$ & 0 & 0 & 0 \\
\hline 0 & 0 & $-3.5000 e+002$ & 0 & 0 \\
\hline 0 & 0 & 0 & $-3.5000 e+002$ & 0 \\
\hline 0 & 0 & 0 & 0 & $-3.5000 e+002$ \\
\hline 0 & 0 & 0 & 0 & 0 \\
\hline $1.3700 e+001$ & 0 & 0 & 0 & 0 \\
\hline 0 & $1.3700 e+001$ & 0 & 0 & 0 \\
\hline 0 & 0 & $1.3700 e+001$ & 0 & 0 \\
\hline 0 & 0 & 0 & $1.3700 e+001$ & 0 \\
\hline 0 & 0 & 0 & 0 & 0 \\
\hline 0 & 0 & 0 & 0 & 0 \\
\hline 0 & 0 & 0 & 0 & 0 \\
\hline 0 & 0 & 0 & 0 & 0 \\
\hline 0 & 0 & 0 & 0 & 0 \\
\hline 0 & 0 & 0 & 0 & 0 \\
\hline 0 & 0 & 0 & 0 & 0 \\
\hline 0 & 0 & 0 & 0 & 0 \\
\hline 0 & 0 & 0 & 0 & 0 \\
\hline 0 & 0 & 0 & 0 & 0 \\
\hline 0 & 0 & 0 & 0 & 0 \\
\hline 0 & 0 & 0 & 0 & 0 \\
\hline 0 & 0 & 0 & 0 & 0 \\
\hline 0 & 0 & 0 & 0 & 0 \\
\hline 0 & 0 & 0 & 0 & 0 \\
\hline
\end{tabular}




$\begin{array}{lllll}0 & 0 & 0 & 0 & 0 \\ 0 & 0 & 0 & 0 & 0 \\ 0 & 0 & 0 & 0 & 0 \\ 0 & 0 & 0 & 0 & 0 \\ 0 & 0 & 0 & 0 & 0 \\ 0 & 0 & 0 & 0 & 0\end{array}$

Columns 16 through 20

\begin{tabular}{|c|c|c|c|c|}
\hline 0 & 0 & 0 & 0 & 0 \\
\hline 0 & 0 & 0 & 0 & 0 \\
\hline 0 & 0 & 0 & 0 & 0 \\
\hline 0 & 0 & 0 & 0 & 0 \\
\hline 0 & 0 & 0 & 0 & 0 \\
\hline 0 & 0 & 0 & 0 & 0 \\
\hline 0 & 0 & 0 & 0 & 0 \\
\hline 0 & 0 & 0 & 0 & 0 \\
\hline 0 & 0 & 0 & 0 & 0 \\
\hline 0 & 0 & 0 & 0 & 0 \\
\hline 0 & 0 & 0 & 0 & 0 \\
\hline 0 & 0 & 0 & 0 & 0 \\
\hline 0 & 0 & 0 & 0 & 0 \\
\hline 0 & 0 & 0 & 0 & 0 \\
\hline 0 & 0 & 0 & 0 & 0 \\
\hline$-1.3200 e+001$ & 0 & 0 & 0 & 0 \\
\hline 0 & $-1.3200 e+001$ & 0 & 0 & 0 \\
\hline 0 & 0 & $-1.3200 e+001$ & 0 & 0 \\
\hline 0 & 0 & 0 & $-1.3200 e+001$ & 0 \\
\hline 0 & 0 & 0 & 0 & $-1.3200 e+001$ \\
\hline 0 & 0 & 0 & 0 & 0 \\
\hline 0 & 0 & 0 & 0 & 0 \\
\hline 0 & 0 & 0 & 0 & 0 \\
\hline 0 & 0 & 0 & 0 & 0 \\
\hline 0 & 0 & 0 & 0 & 0 \\
\hline 0 & 0 & 0 & 0 & 0 \\
\hline $1.5600 e+001$ & 0 & 0 & 0 & 0 \\
\hline 0 & $1.5600 e+001$ & 0 & 0 & 0 \\
\hline 0 & 0 & $1.5600 e+001$ & 0 & 0 \\
\hline 0 & 0 & 0 & $1.5600 e+001$ & 0 \\
\hline 0 & 0 & 0 & 0 & $1.5600 \mathrm{e}+001$ \\
\hline 0 & 0 & 0 & 0 & 0 \\
\hline 0 & 0 & 0 & 0 & 0 \\
\hline 0 & 0 & 0 & 0 & 0 \\
\hline 0 & 0 & 0 & 0 & 0 \\
\hline 0 & 0 & 0 & 0 & 0 \\
\hline 0 & 0 & 0 & 0 & 0 \\
\hline 0 & 0 & 0 & 0 & 0 \\
\hline 0 & 0 & 0 & 0 & 0 \\
\hline 0 & 0 & 0 & 0 & 0 \\
\hline 0 & 0 & 0 & 0 & 0 \\
\hline
\end{tabular}

Columns 21 through 25

0

0

0

0 


\begin{tabular}{|c|c|c|c|c|}
\hline 0 & 0 & 0 & 0 & 0 \\
\hline 0 & 0 & 0 & 0 & 0 \\
\hline 0 & 0 & 0 & 0 & 0 \\
\hline 0 & 0 & 0 & 0 & 0 \\
\hline 0 & 0 & 0 & 0 & 0 \\
\hline 0 & 0 & 0 & 0 & 0 \\
\hline 0 & 0 & 0 & 0 & 0 \\
\hline 0 & 0 & 0 & 0 & 0 \\
\hline 0 & 0 & 0 & 0 & 0 \\
\hline 0 & 0 & 0 & 0 & 0 \\
\hline 0 & 0 & 0 & 0 & 0 \\
\hline 0 & 0 & 0 & 0 & 0 \\
\hline 0 & 0 & 0 & 0 & 0 \\
\hline 0 & 0 & 0 & 0 & 0 \\
\hline 0 & 0 & 0 & 0 & 0 \\
\hline 0 & 0 & 0 & 0 & 0 \\
\hline 0 & 0 & 0 & 0 & 0 \\
\hline 0 & 0 & 0 & 0 & 0 \\
\hline 0 & 0 & 0 & 0 & 0 \\
\hline$-1.6420 e+001$ & 0 & 0 & 0 & 0 \\
\hline 0 & $-1.5600 e+000$ & 0 & 0 & 0 \\
\hline 0 & 0 & $-1.0400 e+000$ & 0 & 0 \\
\hline 0 & 0 & 0 & $-2.0800 e+000$ & 0 \\
\hline 0 & 0 & 0 & 0 & $-3.6400 e+000$ \\
\hline 0 & 0 & 0 & 0 & 0 \\
\hline 0 & $1.4800 e+001$ & 0 & 0 & 0 \\
\hline 0 & 0 & $1.4800 e+001$ & 0 & 0 \\
\hline 0 & 0 & 0 & $1.4800 e+001$ & 0 \\
\hline 0 & 0 & 0 & 0 & $1.4800 e+001$ \\
\hline 0 & 0 & 0 & 0 & 0 \\
\hline 0 & 0 & 0 & 0 & 0 \\
\hline 0 & 0 & 0 & 0 & 0 \\
\hline 0 & 0 & 0 & 0 & 0 \\
\hline 0 & 0 & 0 & 0 & 0 \\
\hline 0 & 0 & 0 & 0 & 0 \\
\hline 0 & 0 & 0 & 0 & 0 \\
\hline 0 & 0 & 0 & 0 & 0 \\
\hline 0 & 0 & 0 & 0 & 0 \\
\hline 0 & 0 & 0 & 0 & 0 \\
\hline 0 & 0 & 0 & 0 & 0 \\
\hline
\end{tabular}

Columns 26 through 30

$\begin{array}{lllll}0 & 0 & 0 & 0 & 0 \\ 0 & 0 & 0 & 0 & 0 \\ 0 & 0 & 0 & 0 & 0 \\ 0 & 0 & 0 & 0 & 0 \\ 0 & 0 & 0 & 0 & 0 \\ 0 & 0 & 0 & 0 & 0 \\ 0 & 0 & 0 & 0 & 0 \\ 0 & 0 & 0 & 0 & 0 \\ 0 & 0 & 0 & 0 & 0 \\ 0 & 0 & 0 & 0 & 0 \\ 0 & 0 & 0 & 0 & 0\end{array}$




\begin{tabular}{|c|c|c|c|c|}
\hline 0 & 0 & 0 & 0 & 0 \\
\hline 0 & 0 & 0 & 0 & 0 \\
\hline 0 & 0 & 0 & 0 & 0 \\
\hline 0 & 0 & 0 & 0 & 0 \\
\hline 0 & 0 & 0 & 0 & 0 \\
\hline 0 & 0 & 0 & 0 & 0 \\
\hline 0 & 0 & 0 & 0 & 0 \\
\hline 0 & 0 & 0 & 0 & 0 \\
\hline 0 & 0 & 0 & 0 & 0 \\
\hline 0 & 0 & 0 & 0 & 0 \\
\hline 0 & $1.5600 \mathrm{e}+000$ & 0 & 0 & 0 \\
\hline 0 & 0 & $1.0400 \mathrm{e}+000$ & 0 & 0 \\
\hline 0 & 0 & 0 & $2.0800 e+000$ & 0 \\
\hline 0 & 0 & 0 & 0 & $3.6400 e+000$ \\
\hline$-3.6400 e+000$ & 0 & 0 & 0 & 0 \\
\hline 0 & $-4.6000 e+001$ & 0 & 0 & 0 \\
\hline 0 & 0 & $-4.6000 e+001$ & 0 & 0 \\
\hline 0 & 0 & 0 & $-4.6000 e+001$ & 0 \\
\hline 0 & 0 & 0 & 0 & $-4.6000 e+001$ \\
\hline $1.4800 e+001$ & 0 & 0 & 0 & 0 \\
\hline 0 & $3.5650 e+001$ & 0 & 0 & 0 \\
\hline 0 & 0 & $3.5650 e+001$ & 0 & 0 \\
\hline 0 & 0 & 0 & $3.5650 e+001$ & 0 \\
\hline 0 & 0 & 0 & 0 & $3.5650 \mathrm{e}+001$ \\
\hline 0 & 0 & 0 & 0 & 0 \\
\hline 0 & 0 & 0 & 0 & 0 \\
\hline 0 & 0 & 0 & 0 & 0 \\
\hline 0 & 0 & 0 & 0 & 0 \\
\hline 0 & 0 & 0 & 0 & 0 \\
\hline 0 & 0 & 0 & 0 & \\
\hline
\end{tabular}

Columns 31 through 35

$\begin{array}{lllll}0 & 0 & 0 & 0 & 0 \\ 0 & 0 & 0 & 0 & 0 \\ 0 & 0 & 0 & 0 & 0 \\ 0 & 0 & 0 & 0 & 0 \\ 0 & 0 & 0 & 0 & 0 \\ 0 & 0 & 0 & 0 & 0 \\ 0 & 0 & 0 & 0 & 0 \\ 0 & 0 & 0 & 0 & 0 \\ 0 & 0 & 0 & 0 & 0 \\ 0 & 0 & 0 & 0 & 0 \\ 0 & 0 & 0 & 0 & 0 \\ 0 & 0 & 0 & 0 & 0 \\ 0 & 0 & 0 & 0 & 0 \\ 0 & 0 & 0 & 0 & 0 \\ 0 & 0 & 0 & 0 & 0 \\ 0 & 0 & 0 & 0 & 0 \\ 0 & 0 & 0 & 0 & 0 \\ 0 & 0 & 0 & 0 & 0 \\ 0 & 0 & 0 & 0 & 0 \\ 0 & 0 & 0 & 0 & 0 \\ 0 & 0 & 0 & 0\end{array}$




\begin{tabular}{|c|c|c|c|c|}
\hline 0 & 0 & 0 & 0 & 0 \\
\hline 0 & 0 & 0 & 0 & 0 \\
\hline 0 & 0 & 0 & 0 & 0 \\
\hline 0 & 0 & 0 & 0 & 0 \\
\hline $3.6400 e+000$ & 0 & 0 & 0 & 0 \\
\hline 0 & $1.5600 \mathrm{e}+001$ & 0 & 0 & 0 \\
\hline 0 & 0 & $1.5600 \mathrm{e}+001$ & 0 & 0 \\
\hline 0 & 0 & 0 & $1.5600 \mathrm{e}+001$ & 0 \\
\hline 0 & 0 & 0 & 0 & $1.5600 \mathrm{e}+001$ \\
\hline$-4.6000 e+001$ & 0 & 0 & 0 & 0 \\
\hline 0 & $-5.3950 e+001$ & 0 & 0 & 0 \\
\hline 0 & 0 & $-5.3950 e+001$ & 0 & 0 \\
\hline 0 & 0 & 0 & $-5.3950 e+001$ & 0 \\
\hline 0 & 0 & 0 & 0 & $-5.3950 e+001$ \\
\hline $3.5650 e+001$ & 0 & 0 & 0 & 0 \\
\hline 0 & $3.5650 e+001$ & 0 & 0 & \\
\hline 0 & 0 & $3.5650 e+001$ & 0 & 0 \\
\hline 0 & 0 & 0 & $3.5650 e+001$ & 0 \\
\hline 0 & 0 & 0 & 0 & $3.5650 e+001$ \\
\hline 0 & 0 & 0 & 0 & \\
\hline
\end{tabular}

Columns 36 through 40

\begin{tabular}{|c|c|c|c|c|}
\hline 0 & $1.0750 e+001$ & c & & \\
\hline 0 & 0 & $1.0750 e+001$ & & \\
\hline 0 & 0 & c & $1.0750 e+00$ & \\
\hline 0 & 0 & 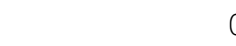 & & $1.0750 \mathrm{e}+00$ \\
\hline 0 & 0 & c & & \\
\hline 0 & 0 & c & & \\
\hline 0 & 0 & c & & \\
\hline 0 & 0 & c & & \\
\hline 0 & 0 & c & & \\
\hline 0 & 0 & c & & \\
\hline 0 & 0 & c & & \\
\hline 0 & 0 & c & & \\
\hline 0 & 0 & c & & \\
\hline 0 & 0 & c & & \\
\hline 0 & 0 & c & & \\
\hline 0 & 0 & c & & \\
\hline 0 & 0 & c & & \\
\hline 0 & 0 & c & & \\
\hline 0 & 0 & c & & \\
\hline 0 & 0 & c & & \\
\hline 0 & 0 & c & & \\
\hline 0 & 0 & c & & \\
\hline 0 & 0 & c & & \\
\hline 0 & 0 & c & & \\
\hline 0 & 0 & c & & \\
\hline 0 & 0 & c & & \\
\hline 0 & 0 & c & & \\
\hline 0 & 0 & c & & \\
\hline 0 & 0 & c & & \\
\hline 0 & 0 & c & & \\
\hline $500 e$ & 0 & c & & \\
\hline
\end{tabular}




$\begin{array}{rrrrr}0 & 1.8300 e+001 & 0 & 0 & 0 \\ 0 & 0 & 1.8300 e+001 & 0 & 0 \\ 0 & 0 & 0 & 1.8300 e+001 & 0 \\ 0 & 0 & 0 & 0 & 1.8300 e+001 \\ -5.3950 e+001 & 0 & 0 & 0 & 0 \\ 0 & -3.5650 e+001 & 0 & 0 & 0 \\ 0 & 0 & 0 & 0 & 0 \\ 0 & 0 & 0 & -3.5650 e+001 & 0 \\ 0 & 0 & 0 & 0 & -3.5650 e+001 \\ 3.5650 e+001 & 0 & 0 & & 0\end{array}$

Column 41

0
0
0
0
$1.0750+0$
0
0
0
0
0
0
0
0
0
0
0
0
0
0
0
0
0
0
0
0
0
0
0
0
0
0
0
0
0
0
0
0
0
0
0
0
0
0
0
0


$\mathcal{B}=$

\begin{tabular}{|c|c|c|c|}
\hline 0 & 0 & 0 & 0 \\
\hline 0 & 0 & 0 & 0 \\
\hline 0 & 0 & 0 & 0 \\
\hline 0 & 0 & 0 & 0 \\
\hline 0 & 0 & 0 & 0 \\
\hline 0 & 0 & 0 & 0 \\
\hline $3.3600 e+000$ & 0 & 0 & 0 \\
\hline 0 & 0 & 0 & 0 \\
\hline 0 & $1.8500 e+002$ & 0 & 0 \\
\hline 0 & 0 & 0 & 0 \\
\hline 0 & 0 & 0 & 0 \\
\hline 0 & 0 & 0 & 0 \\
\hline 0 & 0 & 0 & 0 \\
\hline 0 & 0 & 0 & 0 \\
\hline 0 & 0 & 0 & 0 \\
\hline 0 & 0 & 0 & 0 \\
\hline 0 & 0 & 0 & 0 \\
\hline 0 & 0 & 0 & 0 \\
\hline 0 & 0 & 0 & 0 \\
\hline 0 & 0 & 0 & 0 \\
\hline 0 & 0 & $1.0000 e-002$ & 0 \\
\hline 0 & 0 & 0 & $-1.3300 e-005$ \\
\hline 0 & 0 & 0 & $-4.2100 e-005$ \\
\hline 0 & 0 & 0 & $-2.7000 e-006$ \\
\hline 0 & 0 & 0 & $-3.8100 e-005$ \\
\hline 0 & 0 & 0 & $-3.8000 e-006$ \\
\hline 0 & 0 & 0 & 0 \\
\hline 0 & 0 & 0 & 0 \\
\hline 0 & 0 & 0 & 0 \\
\hline 0 & 0 & 0 & 0 \\
\hline 0 & 0 & 0 & 0 \\
\hline 0 & 0 & 0 & 0 \\
\hline 0 & 0 & 0 & 0 \\
\hline 0 & 0 & 0 & 0 \\
\hline 0 & 0 & 0 & 0 \\
\hline 0 & 0 & 0 & 0 \\
\hline 0 & 0 & 0 & 0 \\
\hline 0 & 0 & 0 & 0 \\
\hline 0 & 0 & 0 & 0 \\
\hline 0 & 0 & 0 & 0 \\
\hline 0 & 0 & 0 & 0 \\
\hline
\end{tabular}

$\mathcal{C}=$

Columns 1 through 11

$\begin{array}{lllllllllll}0 & 0 & 0 & 0 & 0 & 0 & 1 & 0 & 0 & 0 & 0 \\ 0 & 0 & 0 & 0 & 0 & 0 & 0 & 1 & 0 & 0 & 0 \\ 0 & 0 & 0 & 0 & 0 & 0 & 0 & 0 & 0 & 0 & 0\end{array}$




$\begin{array}{llllllllll}0 & 0 & 0 & 0 & 0 & 0 & 0 & 0 & 0 & 0\end{array}$

Columns 12 through 22

$\begin{array}{lllllllllll}0 & 0 & 0 & 0 & 0 & 0 & 0 & 0 & 0 & 0 & 0 \\ 0 & 0 & 0 & 0 & 0 & 0 & 0 & 0 & 0 & 0 & 0 \\ 0 & 0 & 0 & 0 & 0 & 0 & 0 & 0 & 0 & 1 & 0 \\ 0 & 0 & 0 & 0 & 0 & 0 & 0 & 0 & 0 & 0 & 0\end{array}$

Columns 23 through 33

$\begin{array}{lllllllllll}0 & 0 & 0 & 0 & 0 & 0 & 0 & 0 & 0 & 0 & 0 \\ 0 & 0 & 0 & 0 & 0 & 0 & 0 & 0 & 0 & 0 & 0 \\ 0 & 0 & 0 & 0 & 0 & 0 & 0 & 0 & 0 & 0 & 0 \\ 0 & 0 & 0 & 1 & 0 & 0 & 0 & 0 & 0 & 0 & 0\end{array}$

Columns 34 through 41

$\begin{array}{llllllll}0 & 0 & 0 & 0 & 0 & 0 & 0 & 0 \\ 0 & 0 & 0 & 0 & 0 & 0 & 0 & 0 \\ 0 & 0 & 0 & 0 & 0 & 0 & 0 & 0 \\ 0 & 0 & 0 & 0 & 0 & 0 & 0 & 0\end{array}$

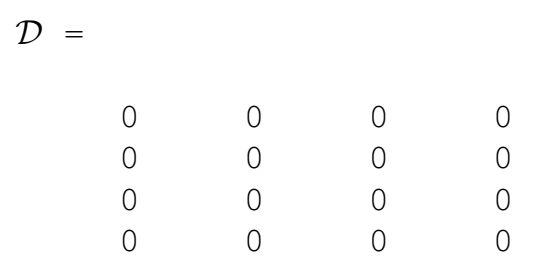

$\begin{array}{rrr}\mathcal{E}= & & \\ 2.1600 e-004 & 0 & 2.8700 e-005 \\ 0 & 2.1600 e-004 & 9.0700 e-005 \\ 0 & 0 & 5.8200 e-006 \\ 0 & 0 & 8.2100 e-005 \\ 0 & 0 & 8.1900 e-006 \\ -1.1500 e-002-1.1500 e-002 & -1.3450 e-002 \\ 0 & 0 & 0 \\ 0 & 0 & 0 \\ 0 & 0 & 0 \\ 0 & 0 & 0 \\ 0 & 0 & 0 \\ 0 & 0 & 0 \\ 0 & 0 & 0 \\ 0 & 0 & 0 \\ 0 & 0 & 0 \\ 0 & 0 & 0 \\ 0 & 0 & 0 \\ 0 & 0 & 0 \\ 0 & 0 & 0\end{array}$




$\begin{array}{rll}\mathcal{F}= & & \\ & & \\ 0 & 0 & 0 \\ 0 & 0 & 0 \\ 0 & 0 & 0 \\ 0 & 0 & 0\end{array}$




\section{Appendix C}

\section{Detailed Optimization Outputs}

This appendix contains the MATLAB optimization outputs for the control design examples presented in Chapters 4 to 8 .

\section{C.1 Control Design for Chemical Plant}

The following optimization outputs were obtained when designing the two-term controller (2.6) for the Williams and Otto chemical plant in Section 5.2.

Design of Controller for the Case $\theta=0$ :

\begin{tabular}{|c|c|c|c|c|}
\hline $\begin{array}{c}\text { Starting KP } \\
0.0005\end{array}$ & $\begin{array}{r}\text { Starting } \\
1 e-005\end{array}$ & $\begin{array}{c}\text { theta } \\
0\end{array}$ & $\begin{array}{c}\mathrm{mu} \\
1 e-006\end{array}$ & $\begin{array}{l}\text { Duration } \mathrm{T} \text { (sec) } \\
\quad 1000\end{array}$ \\
\hline Iteration & Func-count & $\min f(x)$ & & Procedure \\
\hline 0 & 9 & $6.57684 e-006$ & & \\
\hline 1 & 27 & $6.57502 e-006$ & & initial simplex \\
\hline 2 & 45 & $6.57192 e-006$ & & expand \\
\hline 3 & 63 & $6.56965 e-006$ & & expand \\
\hline 4 & 81 & $6.56233 e-006$ & & expand \\
\hline 5 & 99 & $6.55416 e-006$ & & expand \\
\hline 6 & 117 & $6.53553 e-006$ & & expand \\
\hline 7 & 135 & $6.51007 e-006$ & & expand \\
\hline 8 & 153 & $6.46075 e-006$ & & expand \\
\hline 9 & 171 & $6.38686 e-006$ & & expand \\
\hline 10 & 189 & $6.25634 e-006$ & & expand \\
\hline 11 & 207 & $6.05628 e-006$ & & expand \\
\hline 12 & 225 & $5.73105 e-006$ & & expand \\
\hline 13 & 243 & $5.25628 e-006$ & & expand \\
\hline 14 & 261 & $4.58099 e-006$ & & expand \\
\hline 15 & 279 & $3.74244 e-006$ & & expand \\
\hline
\end{tabular}




\begin{tabular}{|c|c|c|c|}
\hline 16 & 297 & $2.8289 e-006$ & expand \\
\hline 17 & 315 & $2.06782 e-006$ & expand \\
\hline 18 & 333 & $1.80004 e-006$ & expand \\
\hline 19 & 342 & $1.80004 e-006$ & reflect \\
\hline 20 & 360 & $1.79615 e-006$ & contract \\
\hline 21 & 396 & $1.78695 e-006$ & shrink \\
\hline 22 & 414 & $1.78695 e-006$ & contract \\
\hline 23 & 432 & $1.78695 e-006$ & contract \\
\hline 24 & 468 & $1.78679 e-006$ & shrink \\
\hline 25 & 504 & $1.78678 e-006$ & shrink \\
\hline 26 & 522 & $1.7867 e-006$ & contract \\
\hline 27 & 540 & $1.7867 e-006$ & contract \\
\hline 28 & 558 & $1.78652 e-006$ & expand \\
\hline 29 & 567 & $1.78652 e-006$ & reflect \\
\hline 30 & 585 & $1.78625 e-006$ & expand \\
\hline 31 & 603 & $1.78602 e-006$ & expand \\
\hline 32 & 621 & $1.78526 e-006$ & expand \\
\hline 33 & 639 & $1.78474 e-006$ & expand \\
\hline 34 & 657 & $1.78262 e-006$ & expand \\
\hline 35 & 675 & $1.78211 e-006$ & expand \\
\hline 36 & 693 & $1.77708 e-006$ & expand \\
\hline 37 & 702 & $1.77708 e-006$ & reflect \\
\hline 38 & 720 & $1.77176 e-006$ & expand \\
\hline 39 & 738 & $1.76241 e-006$ & expand \\
\hline 40 & 756 & $1.75227 e-006$ & expand \\
\hline 41 & 774 & $1.723 e-006$ & expand \\
\hline 42 & 792 & $1.71363 e-006$ & expand \\
\hline 43 & 810 & $1.64124 e-006$ & expand \\
\hline 44 & 819 & $1.64124 e-006$ & reflect \\
\hline 45 & 837 & $1.57286 e-006$ & expand \\
\hline 46 & 855 & $1.45823 e-006$ & expand \\
\hline 47 & 873 & $1.38373 e-006$ & expand \\
\hline 48 & 891 & $1.11937 e-006$ & expand \\
\hline 49 & 900 & $1.11937 e-006$ & reflect \\
\hline 50 & 918 & $9.02927 e-007$ & expand \\
\hline 51 & 936 & $8.4313 e-007$ & expand \\
\hline 52 & 954 & $4.96447 e-007$ & expand \\
\hline 53 & 963 & $4.96447 e-007$ & reflect \\
\hline 54 & 981 & $3.3263 e-007$ & expand \\
\hline 55 & 999 & $3.3263 e-007$ & contract \\
\hline 56 & 1017 & $2.21986 e-007$ & expand \\
\hline 57 & 1026 & $2.21986 e-007$ & reflect \\
\hline 58 & 1044 & $2.13742 e-007$ & expand \\
\hline 59 & 1062 & $2.10425 e-007$ & contract \\
\hline 60 & 1080 & $2.03483 e-007$ & contract \\
\hline 61 & 1098 & $1.99425 e-007$ & contract \\
\hline 62 & 1116 & $1.99425 e-007$ & contract \\
\hline 63 & 1134 & $1.97915 e-007$ & reflect \\
\hline 64 & 1152 & $1.97469 e-007$ & contract \\
\hline 65 & 1170 & $1.97169 e-007$ & contract \\
\hline 66 & 1188 & $1.97026 e-007$ & contract \\
\hline 67 & 1206 & $1.96974 \mathrm{e}-007$ & reflect \\
\hline 68 & 1224 & $1.96879 e-007$ & contract \\
\hline 69 & 1242 & $1.96819 e-007$ & contract \\
\hline
\end{tabular}




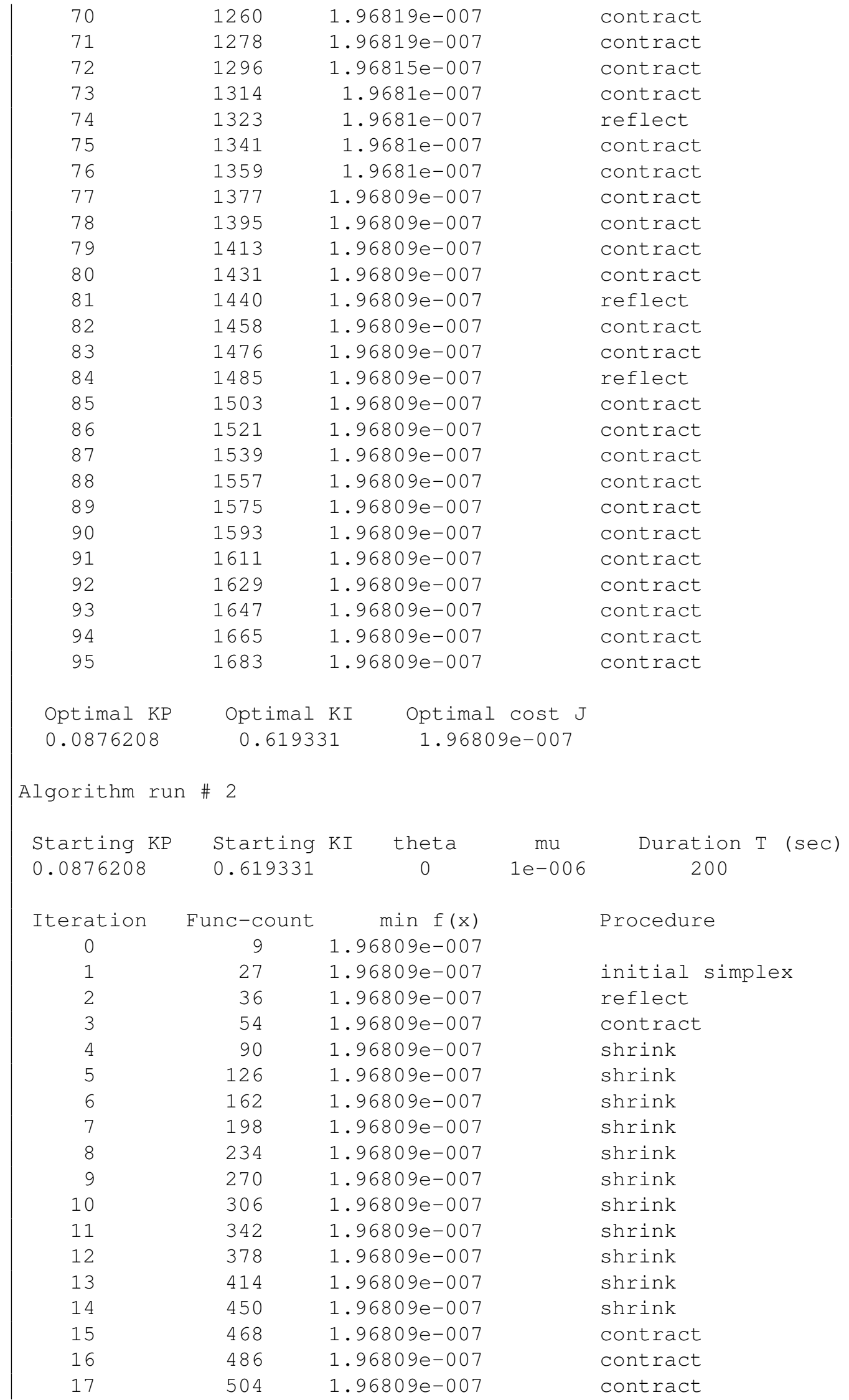


Optimal KP Optimal KI Optimal cost J
0.0876208
0.619331
$1.96809 e-007$

Design of Controller for the Case $\theta=20$ :

\begin{tabular}{|c|c|c|c|c|}
\hline \multicolumn{5}{|c|}{ Algorithm run \# 1} \\
\hline $\begin{array}{c}\text { Starting KP } \\
0.0005\end{array}$ & $\begin{array}{r}\text { Starting } \\
1 e-005\end{array}$ & $\begin{array}{c}\text { theta } \\
20\end{array}$ & $\begin{array}{c}\mathrm{mu} \\
1 e-006\end{array}$ & $\begin{array}{c}\text { Duration } \mathrm{T} \text { (sec) } \\
1000\end{array}$ \\
\hline Iteration & Func-count & $\min f(x)$ & & Procedure \\
\hline 0 & 9 & 0.000827682 & & \\
\hline 1 & 27 & 0.000827632 & & initial simplex \\
\hline 2 & 45 & 0.000827568 & & expand \\
\hline 3 & 63 & 0.000827488 & & expand \\
\hline 4 & 81 & 0.00082732 & & expand \\
\hline 5 & 99 & 0.000827077 & & expand \\
\hline 6 & 117 & 0.000826621 & & expand \\
\hline 7 & 135 & 0.00082591 & & expand \\
\hline 8 & 153 & 0.00082465 & & expand \\
\hline 9 & 171 & 0.00082262 & & expand \\
\hline 10 & 189 & 0.000819151 & & expand \\
\hline 11 & 207 & 0.000813532 & & expand \\
\hline 12 & 225 & 0.000804274 & & expand \\
\hline 13 & 243 & 0.0007897 & & expand \\
\hline 14 & 261 & 0.000767263 & & expand \\
\hline 15 & 279 & 0.000735047 & & expand \\
\hline 16 & 297 & 0.000692522 & & expand \\
\hline 17 & 315 & 0.000644765 & & expand \\
\hline 18 & 333 & 0.000619763 & & expand \\
\hline 19 & 369 & 0.000619763 & & shrink \\
\hline 20 & 405 & 0.000617309 & & shrink \\
\hline 21 & 423 & 0.000617086 & & contract \\
\hline 22 & 459 & 0.00061708 & & shrink \\
\hline 23 & 495 & 0.000617052 & & shrink \\
\hline 24 & 513 & 0.000617052 & & contract \\
\hline 25 & 531 & 0.000617052 & & contract \\
\hline 26 & 549 & 0.000617052 & & contract \\
\hline 27 & 567 & 0.000617052 & & contract \\
\hline 28 & 603 & 0.000617052 & & shrink \\
\hline 29 & 639 & 0.000617052 & & shrink \\
\hline 30 & 675 & 0.000617052 & & shrink \\
\hline 31 & 711 & 0.000617052 & & shrink \\
\hline 32 & 729 & 0.000617052 & & contract \\
\hline 33 & 747 & 0.000617052 & & contract \\
\hline 34 & 765 & 0.000617052 & & expand \\
\hline 35 & 774 & 0.000617052 & & reflect \\
\hline 36 & 792 & 0.000617052 & & expand \\
\hline 37 & 810 & 0.000617052 & & expand \\
\hline 38 & 828 & 0.000617052 & & expand \\
\hline 39 & 846 & 0.000617052 & & expand \\
\hline 40 & 864 & 0.000617052 & & expand \\
\hline
\end{tabular}




\begin{tabular}{|c|c|c|c|}
\hline 41 & 882 & 0.000617052 & expand \\
\hline 42 & 900 & 0.000617051 & expand \\
\hline 43 & 909 & 0.000617051 & reflect \\
\hline 44 & 927 & 0.000617051 & expand \\
\hline 45 & 945 & 0.000617051 & expand \\
\hline 46 & 963 & 0.000617051 & expand \\
\hline 47 & 981 & 0.00061705 & expand \\
\hline 48 & 999 & 0.000617049 & expand \\
\hline 49 & 1017 & 0.000617048 & expand \\
\hline 50 & 1035 & 0.000617045 & expand \\
\hline 51 & 1053 & 0.000617041 & expand \\
\hline 52 & 1071 & 0.000617035 & expand \\
\hline 53 & 1089 & 0.000617023 & expand \\
\hline 54 & 1107 & 0.000617013 & expand \\
\hline 55 & 1125 & 0.000616986 & expand \\
\hline 56 & 1134 & 0.000616986 & reflect \\
\hline 57 & 1152 & 0.000616967 & expand \\
\hline 58 & 1170 & 0.000616925 & expand \\
\hline 59 & 1179 & 0.000616925 & reflect \\
\hline 60 & 1197 & 0.000616859 & expand \\
\hline 61 & 1215 & 0.000616847 & expand \\
\hline 62 & 1233 & 0.000616701 & expand \\
\hline 63 & 1242 & 0.000616701 & reflect \\
\hline 64 & 1260 & 0.000616643 & expand \\
\hline 65 & 1278 & 0.000616496 & expand \\
\hline 66 & 1287 & 0.000616496 & reflect \\
\hline 67 & 1305 & 0.000616466 & expand \\
\hline 68 & 1323 & 0.000616442 & contract \\
\hline 69 & 1341 & 0.00061643 & reflect \\
\hline 70 & 1359 & 0.000616422 & contract \\
\hline 71 & 1377 & 0.000616418 & contract \\
\hline 72 & 1395 & 0.000616414 & contract \\
\hline 73 & 1413 & 0.000616414 & contract \\
\hline 74 & 1431 & 0.000616414 & contract \\
\hline 75 & 1449 & 0.000616414 & reflect \\
\hline 76 & 1467 & 0.000616414 & contract \\
\hline 77 & 1485 & 0.000616414 & contract \\
\hline 78 & 1503 & 0.000616414 & contract \\
\hline 79 & 1521 & 0.000616414 & contract \\
\hline 80 & 1539 & 0.000616414 & contract \\
\hline 81 & 1557 & 0.000616414 & reflect \\
\hline 82 & 1575 & 0.000616414 & contract \\
\hline 83 & 1593 & 0.000616414 & contract \\
\hline 84 & 1611 & 0.000616414 & contract \\
\hline 85 & 1629 & 0.000616414 & contract \\
\hline 86 & 1647 & 0.000616414 & contract \\
\hline 87 & 1683 & 0.000616414 & shrink \\
\hline 88 & 1701 & 0.000616414 & contract \\
\hline 89 & 1719 & 0.000616414 & contract \\
\hline 90 & 1728 & 0.000616414 & reflect \\
\hline 91 & 1746 & 0.000616414 & contract \\
\hline 92 & 1764 & 0.000616414 & contract \\
\hline 93 & 1782 & 0.000616414 & contract \\
\hline 94 & 1800 & 0.000616414 & contract \\
\hline
\end{tabular}




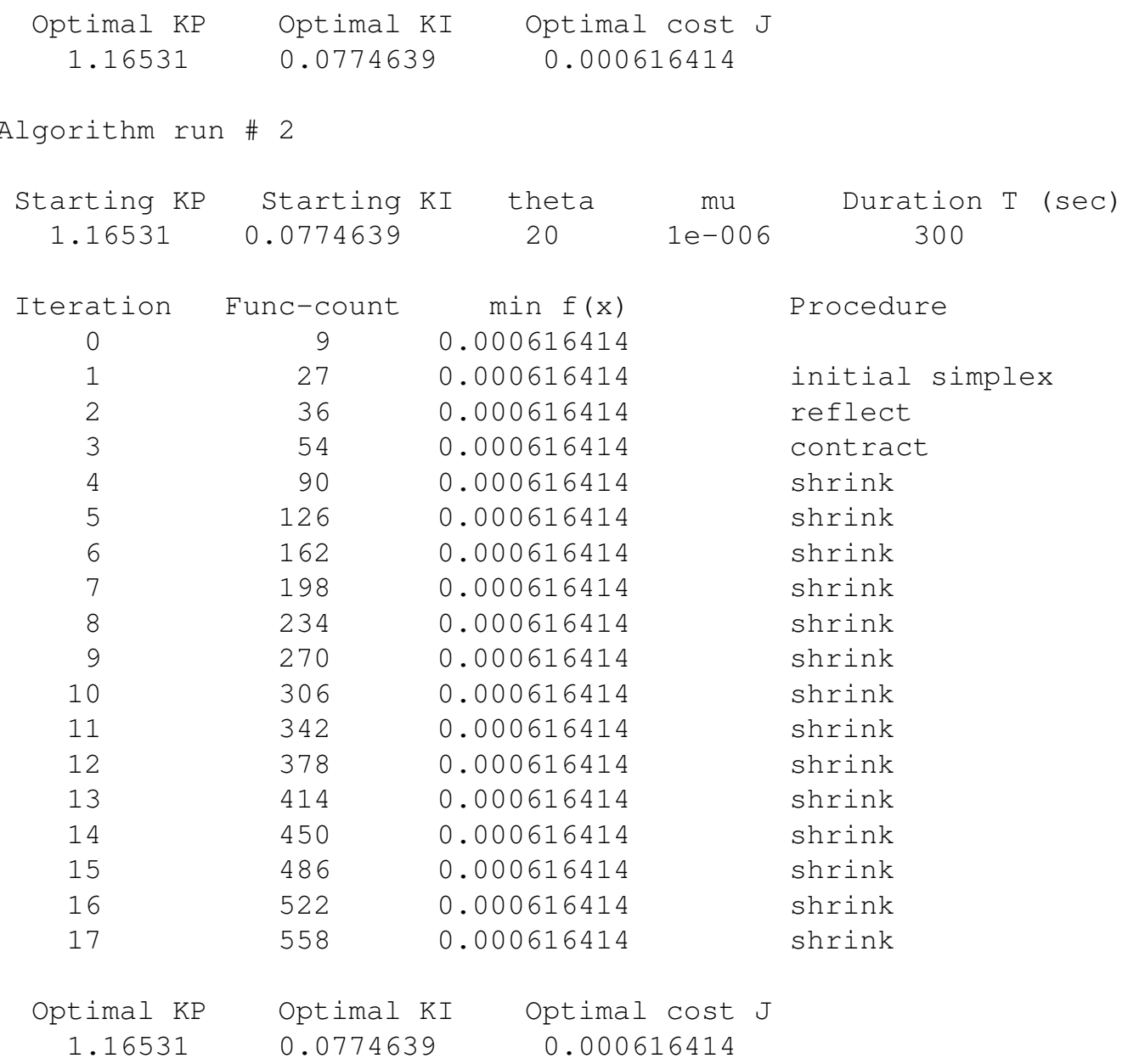

\section{C.2 Control Design for Turbo-Generator}

The following optimization outputs were obtained when designing the two-term controller (2.6) for the Turbo-Generator in Sections 6.2 and 6.3 .

$\underline{\text { Design } 1 \text { with } \theta=0 \text { : }}$

\begin{tabular}{|ccccc}
\hline Algorithm run \# & & & \\
Starting KP & Starting KI & theta & mu & Duration $\mathrm{T}$ (sec) \\
0.0005 & le-005 & 0 & 0.0001 & 10000 \\
Iteration & Func-count & min $\mathrm{f}(\mathrm{x})$ & Procedure \\
0 & 4 & 496.693 & \\
1 & 12 & 495.633 & initial simplex \\
2 & 20 & 494.376 & expand
\end{tabular}




\begin{tabular}{|c|c|c|c|}
\hline 3 & 28 & 492.622 & expand \\
\hline 4 & 36 & 489.283 & expand \\
\hline 5 & 44 & 484.231 & expand \\
\hline 6 & 52 & 475.415 & expand \\
\hline 7 & 60 & 461.875 & expand \\
\hline 8 & 68 & 440.139 & expand \\
\hline 9 & 76 & 408.556 & expand \\
\hline 10 & 84 & 364.228 & expand \\
\hline 11 & 92 & 308.999 & expand \\
\hline 12 & 100 & 246.97 & expand \\
\hline 13 & 108 & 186.435 & expand \\
\hline 14 & 116 & 134.291 & expand \\
\hline 15 & 124 & 94.1337 & expand \\
\hline 16 & 132 & 65.8417 & expand \\
\hline 17 & 140 & 46.9734 & expand \\
\hline 18 & 148 & 41.3391 & reflect \\
\hline 19 & 164 & 41.3391 & shrink \\
\hline 20 & 180 & 41.3391 & shrink \\
\hline 21 & 188 & 39.5219 & reflect \\
\hline 22 & 196 & 38.5712 & reflect \\
\hline 23 & 212 & 38.5712 & shrink \\
\hline 24 & 228 & 38.5712 & shrink \\
\hline 25 & 244 & 38.5712 & shrink \\
\hline 26 & 252 & 38.3787 & reflect \\
\hline 27 & 268 & 38.3787 & shrink \\
\hline 28 & 276 & 38.3238 & reflect \\
\hline 29 & 292 & 38.3238 & shrink \\
\hline 30 & 308 & 38.3238 & shrink \\
\hline 31 & 324 & 38.3238 & shrink \\
\hline 32 & 332 & 38.3192 & reflect \\
\hline 33 & 348 & 38.3192 & shrink \\
\hline 34 & 364 & 38.3183 & shrink \\
\hline 35 & 372 & 38.3183 & contract \\
\hline 36 & 388 & 38.3182 & shrink \\
\hline 37 & 404 & 38.3182 & shrink \\
\hline 38 & 420 & 38.3182 & shrink \\
\hline 39 & 428 & 38.3182 & contract \\
\hline Optimal KP & Optimal KI & Optimal cost $\mathrm{J}$ & \\
\hline 0.667782 & 0.0212588 & 38.3182 & \\
\hline Algorithm run & $n \# 2$ & & \\
\hline Starting KP & Starting $\mathrm{KI}$ & theta & Duration $\mathrm{T}$ (sec) \\
\hline 0.667782 & 0.0212588 & 0.0001 & 1000 \\
\hline Iteration & Func-count & $\min f(x)$ & Procedure \\
\hline 0 & 4 & 38.3134 & \\
\hline 1 & 12 & 38.3134 & initial simplex \\
\hline 2 & 28 & 38.3134 & shrink \\
\hline 3 & 44 & 38.3134 & shrink \\
\hline 4 & 52 & 36.354 & expand \\
\hline 5 & 56 & 36.354 & reflect \\
\hline 6 & 64 & 34.6553 & expand \\
\hline
\end{tabular}




\begin{tabular}{|c|c|c|}
\hline 72 & 31.329 & expand \\
\hline 80 & 26.4811 & expand \\
\hline 88 & 19.9677 & expand \\
\hline 96 & 10.7447 & expand \\
\hline 104 & 9.2205 & reflect \\
\hline 120 & 9.2205 & shrink \\
\hline 136 & 9.2205 & shrink \\
\hline 152 & 9.2205 & shrink \\
\hline 168 & 9.2205 & shrink \\
\hline 176 & 9.20384 & contract \\
\hline 184 & 9.16041 & expand \\
\hline 188 & 9.16041 & reflect \\
\hline 196 & 9.10263 & expand \\
\hline 204 & 9.07892 & expand \\
\hline 212 & 8.88061 & expand \\
\hline 216 & 8.88061 & reflect \\
\hline 224 & 8.56901 & expand \\
\hline 232 & 8.50489 & expand \\
\hline 240 & 7.84742 & expand \\
\hline 244 & 7.84742 & reflect \\
\hline 252 & 7.11033 & expand \\
\hline 260 & 6.55647 & expand \\
\hline 268 & 5.67518 & expand \\
\hline 276 & 4.8346 & expand \\
\hline 284 & 4.17743 & expand \\
\hline 292 & 3.40633 & expand \\
\hline 300 & 3.40633 & contract \\
\hline 308 & 3.0921 & expand \\
\hline 316 & 2.78411 & reflect \\
\hline 324 & 2.3917 & expand \\
\hline 332 & 2.3917 & contract \\
\hline 336 & 2.3917 & reflect \\
\hline 344 & 2.22491 & expand \\
\hline 352 & 1.90301 & expand \\
\hline 356 & 1.90301 & reflect \\
\hline 364 & 1.72055 & reflect \\
\hline 372 & 1.72055 & contract \\
\hline 376 & 1.72055 & reflect \\
\hline 384 & 1.64056 & expand \\
\hline 392 & 1.64056 & contract \\
\hline 400 & 1.60404 & reflect \\
\hline 408 & 1.60404 & contract \\
\hline 416 & 1.59117 & contract \\
\hline 424 & 1.59117 & contract \\
\hline 432 & 1.59117 & contract \\
\hline 440 & 1.59117 & contract \\
\hline 444 & 1.59117 & reflect \\
\hline 448 & 1.59117 & reflect \\
\hline 456 & 1.59074 & contract \\
\hline 464 & 1.59068 & contract \\
\hline 472 & 1.59065 & contract \\
\hline 480 & 1.59056 & contract \\
\hline 488 & 1.59056 & contract \\
\hline 496 & 1.59056 & contract \\
\hline
\end{tabular}




\begin{tabular}{|c|c|c|c|}
\hline 61 & 504 & 1.59055 & contract \\
\hline 62 & 512 & 1.59055 & contract \\
\hline 63 & 520 & 1.59055 & contract \\
\hline 64 & 528 & 1.59055 & contract \\
\hline 65 & 532 & 1.59055 & reflect \\
\hline 66 & 540 & 1.59055 & contract \\
\hline 67 & 548 & 1.59055 & contract \\
\hline 68 & 556 & 1.59055 & contract \\
\hline 69 & 564 & 1.59055 & contract \\
\hline 70 & 572 & 1.59055 & contract \\
\hline 71 & 580 & 1.59055 & contract \\
\hline 72 & 588 & 1.59055 & contract \\
\hline 73 & 596 & 1.59055 & contract \\
\hline 74 & 604 & 1.59055 & contract \\
\hline 75 & 612 & 1.59055 & contract \\
\hline 76 & 620 & 1.59055 & contract \\
\hline 77 & 628 & 1.59055 & contract \\
\hline 78 & 636 & 1.59055 & contract \\
\hline 79 & 644 & 1.59055 & contract \\
\hline 80 & 652 & 1.59055 & contract \\
\hline 81 & 660 & 1.59055 & contract \\
\hline 82 & 664 & 1.59055 & reflect \\
\hline 83 & 672 & 1.59055 & contract \\
\hline 84 & 680 & 1.59055 & contract \\
\hline $\begin{array}{l}\text { Optimal KP } \\
0.712985\end{array}$ & $\begin{array}{c}\text { Optimal KI } \\
2.00619\end{array}$ & $\begin{array}{c}\text { Optimal cost } \mathrm{J} \\
1.59055\end{array}$ & \\
\hline Algorithm run & $n \# 3$ & & \\
\hline $\begin{array}{l}\text { Starting KP } \\
0.712985\end{array}$ & $\begin{array}{l}\text { Starting KI } \\
2.00619\end{array}$ & $\begin{array}{cc}\text { theta } & \mathrm{mu} \\
0 & 0.0001\end{array}$ & $\begin{array}{c}\text { Duration } \mathrm{T} \text { (sec) } \\
300\end{array}$ \\
\hline Iteration & Func-count & $\min f(x)$ & Procedure \\
\hline 0 & 4 & 1.59055 & \\
\hline 1 & 12 & 1.59055 & initial simplex \\
\hline 2 & 16 & 1.59055 & reflect \\
\hline 3 & 24 & 1.59055 & contract \\
\hline 4 & 28 & 1.59055 & reflect \\
\hline 5 & 44 & 1.59055 & shrink \\
\hline 6 & 60 & 1.59055 & shrink \\
\hline 7 & 76 & 1.59055 & shrink \\
\hline 8 & 92 & 1.59055 & shrink \\
\hline 9 & 108 & 1.59055 & shrink \\
\hline 10 & 124 & 1.59055 & shrink \\
\hline 11 & 140 & 1.59055 & shrink \\
\hline 12 & 156 & 1.59055 & shrink \\
\hline 13 & 172 & 1.59055 & shrink \\
\hline 14 & 188 & 1.59055 & shrink \\
\hline 15 & 204 & 1.59055 & shrink \\
\hline 16 & 220 & 1.59055 & shrink \\
\hline 17 & 236 & 1.59055 & shrink \\
\hline 18 & 252 & 1.59055 & shrink \\
\hline 19 & 268 & 1.59055 & shrink \\
\hline
\end{tabular}


Optimal KP Optimal KI Optimal cost J
0.712985
2.00619
1.59055

Design 1 with $\theta=10$ :

\begin{tabular}{|c|c|c|c|c|}
\hline \multicolumn{5}{|c|}{ Algorithm run \# 1} \\
\hline $\begin{array}{c}\text { Starting KP } \\
0.0005\end{array}$ & $\begin{array}{r}\text { Starting KI } \\
1 e-005\end{array}$ & theta & mu & $\begin{array}{l}\text { Duration } \mathrm{T} \text { (sec) } \\
10000\end{array}$ \\
\hline Iteration & Func-count & $\min \mathrm{f}(\mathrm{x})$ & & Procedure \\
\hline 0 & 4 & 486.743 & & \\
\hline 1 & 12 & 485.683 & & initial simplex \\
\hline 2 & 20 & 484.426 & & expand \\
\hline 3 & 28 & 482.672 & & expand \\
\hline 4 & 36 & 479.333 & & expand \\
\hline 5 & 44 & 474.282 & & expand \\
\hline 6 & 52 & 465.467 & & expand \\
\hline 7 & 60 & 451.929 & & expand \\
\hline 8 & 68 & 430.196 & & expand \\
\hline 9 & 76 & 398.617 & & expand \\
\hline 10 & 84 & 354.296 & & expand \\
\hline 11 & 92 & 299.08 & & expand \\
\hline 12 & 100 & 237.071 & & expand \\
\hline 13 & 108 & 176.569 & & expand \\
\hline 14 & 116 & 124.478 & & expand \\
\hline 15 & 124 & 84.4005 & & expand \\
\hline 16 & 132 & 56.2252 & & expand \\
\hline 17 & 140 & 37.5146 & & expand \\
\hline 18 & 148 & 31.9591 & & reflect \\
\hline 19 & 164 & 31.9591 & & shrink \\
\hline 20 & 180 & 31.9591 & & shrink \\
\hline 21 & 188 & 30.1804 & & reflect \\
\hline 22 & 196 & 29.3101 & & reflect \\
\hline 23 & 212 & 29.3101 & & shrink \\
\hline 24 & 228 & 29.3101 & & shrink \\
\hline 25 & 244 & 29.3101 & & shrink \\
\hline 26 & 248 & 29.3101 & & reflect \\
\hline 27 & 256 & 29.3101 & & contract \\
\hline 28 & 264 & 29.265 & & contract \\
\hline 29 & 268 & 29.265 & & reflect \\
\hline 30 & 284 & 29.265 & & shrink \\
\hline 31 & 300 & 29.265 & & shrink \\
\hline 32 & 308 & 29.2649 & & reflect \\
\hline 33 & 316 & 29.2641 & & reflect \\
\hline 34 & 324 & 29.2637 & & contract \\
\hline 35 & 332 & 29.2635 & & reflect \\
\hline 36 & 340 & 29.2635 & & contract \\
\hline 37 & 348 & 29.2628 & & expand \\
\hline 38 & 352 & 29.2628 & & reflect \\
\hline 39 & 360 & 29.2615 & & expand \\
\hline 40 & 364 & 29.2615 & & reflect \\
\hline
\end{tabular}




\begin{tabular}{|c|c|c|c|}
\hline 41 & 372 & 29.261 & expand \\
\hline 42 & 380 & 29.258 & expand \\
\hline 43 & 384 & 29.258 & reflect \\
\hline 44 & 392 & 29.2519 & expand \\
\hline 45 & 396 & 29.2519 & reflect \\
\hline 46 & 404 & 29.2412 & expand \\
\hline 47 & 412 & 29.2343 & expand \\
\hline 48 & 420 & 29.2075 & expand \\
\hline 49 & 428 & 29.1872 & expand \\
\hline 50 & 436 & 29.1157 & expand \\
\hline 51 & 444 & 29.1008 & expand \\
\hline 52 & 452 & 28.9211 & expand \\
\hline 53 & 460 & 28.9211 & contract \\
\hline 54 & 468 & 28.774 & expand \\
\hline 55 & 476 & 28.4249 & expand \\
\hline 56 & 484 & 28.0376 & expand \\
\hline 57 & 492 & 27.1478 & expand \\
\hline 58 & 500 & 26.1245 & expand \\
\hline 59 & 508 & 24.1048 & expand \\
\hline 60 & 516 & 21.6709 & expand \\
\hline 61 & 524 & 17.8847 & expand \\
\hline 62 & 532 & 13.6857 & expand \\
\hline 63 & 540 & 8.92275 & expand \\
\hline 64 & 548 & 4.82966 & expand \\
\hline 65 & 556 & 1.96297 & expand \\
\hline 66 & 564 & 1.32873 & expand \\
\hline 67 & 572 & 1.32873 & contract \\
\hline 68 & 580 & 1.25181 & contract \\
\hline 69 & 584 & 1.25181 & reflect \\
\hline 70 & 592 & 1.2166 & contract \\
\hline 71 & 596 & 1.2166 & reflect \\
\hline 72 & 604 & 1.21398 & contract \\
\hline 73 & 612 & 1.21398 & contract \\
\hline 74 & 620 & 1.21312 & reflect \\
\hline 75 & 628 & 1.2107 & contract \\
\hline 76 & 636 & 1.20909 & expand \\
\hline 77 & 644 & 1.20909 & contract \\
\hline 78 & 652 & 1.20613 & expand \\
\hline 79 & 656 & 1.20613 & reflect \\
\hline 80 & 664 & 1.20096 & expand \\
\hline 81 & 668 & 1.20096 & reflect \\
\hline 82 & 676 & 1.19688 & expand \\
\hline 83 & 684 & 1.19642 & reflect \\
\hline 84 & 700 & 1.19617 & shrink \\
\hline 85 & 708 & 1.19617 & contract \\
\hline 86 & 716 & 1.19617 & contract \\
\hline 87 & 724 & 1.19614 & contract \\
\hline 88 & 732 & 1.19612 & contract \\
\hline 89 & 740 & 1.19612 & contract \\
\hline 90 & 748 & 1.19612 & contract \\
\hline 91 & 756 & 1.19611 & contract \\
\hline 92 & 764 & 1.19611 & contract \\
\hline 93 & 772 & 1.19611 & contract \\
\hline 94 & 776 & 1.19611 & reflect \\
\hline
\end{tabular}




\begin{tabular}{|c|c|c|c|}
\hline 95 & 784 & 1.19611 & contract \\
\hline 96 & 792 & 1.19611 & contract \\
\hline 97 & 800 & 1.19611 & contract \\
\hline 98 & 808 & 1.19611 & contract \\
\hline 99 & 816 & 1.19611 & contract \\
\hline 100 & 824 & 1.19611 & contract \\
\hline 101 & 832 & 1.19611 & contract \\
\hline 102 & 840 & 1.19611 & contract \\
\hline 103 & 848 & 1.19611 & contract \\
\hline 104 & 856 & 1.19611 & contract \\
\hline 105 & 860 & 1.19611 & reflect \\
\hline 106 & 868 & 1.19611 & contract \\
\hline 107 & 876 & 1.19611 & contract \\
\hline Optimal KP & Optimal KI & Optimal cost $\mathrm{J}$ & \\
\hline 0.451414 & 0.137636 & 1.19611 & \\
\hline Algorithm run & \# 2 & & \\
\hline Starting KP & Starting KI & theta & Duration $\mathrm{T}$ (sec) \\
\hline 0.451414 & 0.137636 & 0.0001 & 1000 \\
\hline Iteration & Func-count & $\min f(x)$ & Procedure \\
\hline 0 & 4 & 1.19611 & \\
\hline 1 & 12 & 1.19611 & initial simplex \\
\hline 2 & 20 & 1.19611 & contract \\
\hline 3 & 28 & 1.19611 & contract \\
\hline 4 & 36 & 1.19611 & contract \\
\hline 5 & 40 & 1.19611 & reflect \\
\hline 6 & 48 & 1.19611 & contract \\
\hline 7 & 56 & 1.19611 & contract \\
\hline 8 & 64 & 1.19611 & contract \\
\hline 9 & 68 & 1.19611 & reflect \\
\hline 10 & 76 & 1.19611 & contract \\
\hline 11 & 84 & 1.19611 & contract \\
\hline 12 & 92 & 1.19611 & contract \\
\hline 13 & 100 & 1.19611 & contract \\
\hline 14 & 108 & 1.19611 & contract \\
\hline 15 & 116 & 1.19611 & contract \\
\hline 16 & 124 & 1.19611 & contract \\
\hline 17 & 132 & 1.19611 & contract \\
\hline 18 & 140 & 1.19611 & contract \\
\hline 19 & 148 & 1.19611 & contract \\
\hline 20 & 156 & 1.19611 & contract \\
\hline 21 & 164 & 1.19611 & contract \\
\hline 22 & 172 & 1.19611 & contract \\
\hline 23 & 180 & 1.19611 & contract \\
\hline 24 & 188 & 1.19611 & contract \\
\hline 25 & 196 & 1.19611 & contract \\
\hline 26 & 204 & 1.19611 & contract \\
\hline 27 & 212 & 1.19611 & contract \\
\hline 28 & 216 & 1.19611 & reflect \\
\hline 29 & 224 & 1.19611 & contract \\
\hline 30 & 232 & 1.19611 & contract \\
\hline
\end{tabular}


$31240 \quad 1.19611$ contract

Optimal KP Optimal KI Optimal cost J

$0.451414 \quad 0.137636 \quad 1.19611$

Design 2 with $\theta=0$ :

\begin{tabular}{|c|c|c|c|c|}
\hline \multicolumn{5}{|c|}{ gorithm run \# 1} \\
\hline $\begin{array}{c}\text { Starting KP } \\
0.0005\end{array}$ & $\begin{array}{l}\text { Starting KI } \\
1 e-005\end{array}$ & $\begin{array}{c}\text { theta } \\
0\end{array}$ & $\begin{array}{c}\mathrm{mu} \\
0.0001\end{array}$ & $\begin{array}{l}\text { Duration } \mathrm{T} \text { (sec) } \\
15000\end{array}$ \\
\hline Iteration & Func-count & $\min f(x)$ & & Procedure \\
\hline 0 & 4 & 496.693 & & \\
\hline 1 & 12 & 495.633 & & initial simplex \\
\hline 2 & 20 & 494.376 & & expand \\
\hline 3 & 28 & 492.621 & & expand \\
\hline 4 & 36 & 489.282 & & expand \\
\hline 5 & 44 & 484.231 & & expand \\
\hline 6 & 52 & 475.415 & & expand \\
\hline 7 & 60 & 461.875 & & expand \\
\hline 8 & 68 & 440.139 & & expand \\
\hline 9 & 76 & 408.556 & & expand \\
\hline 10 & 84 & 364.228 & & expand \\
\hline 11 & 92 & 308.999 & & expand \\
\hline 12 & 100 & 246.97 & & expand \\
\hline 13 & 108 & 186.435 & & expand \\
\hline 14 & 116 & 134.291 & & expand \\
\hline 15 & 124 & 94.1337 & & expand \\
\hline 16 & 132 & 65.8417 & & expand \\
\hline 17 & 140 & 46.9734 & & expand \\
\hline 18 & 148 & 41.3391 & & reflect \\
\hline 19 & 164 & 41.3391 & & shrink \\
\hline 20 & 180 & 41.3391 & & shrink \\
\hline 21 & 188 & 39.5219 & & reflect \\
\hline 22 & 196 & 38.5712 & & reflect \\
\hline 23 & 212 & 38.5712 & & shrink \\
\hline 24 & 228 & 38.5712 & & shrink \\
\hline 25 & 244 & 38.5712 & & shrink \\
\hline 26 & 252 & 38.3787 & & reflect \\
\hline 27 & 268 & 38.3787 & & shrink \\
\hline 28 & 276 & 38.324 & & reflect \\
\hline 29 & 292 & 38.324 & & shrink \\
\hline 30 & 308 & 38.324 & & shrink \\
\hline 31 & 324 & 38.324 & & shrink \\
\hline 32 & 340 & 38.324 & & shrink \\
\hline 33 & 348 & 38.3214 & & reflect \\
\hline 34 & 364 & 38.3214 & & shrink \\
\hline 35 & 372 & 38.3214 & & contract \\
\hline 36 & 380 & 38.3214 & & contract \\
\hline 37 & 388 & 38.3213 & & contract \\
\hline 38 & 396 & 38.3213 & & contract \\
\hline 39 & 400 & 38.3213 & & reflect \\
\hline
\end{tabular}




\begin{tabular}{|c|c|c|c|}
\hline 40 & 408 & 38.3213 & contract \\
\hline 41 & 416 & 38.3213 & contract \\
\hline Optimal KP & Optimal KI & Optimal cost $\mathrm{J}$ & \\
\hline 0.667647 & 0.0212544 & 38.3213 & \\
\hline Algorithm run & \# 2 & & \\
\hline Starting $\mathrm{KP}$ & Starting $\mathrm{KI}$ & theta & Duration $\mathrm{T}$ (sec) \\
\hline 0.667647 & 0.0212544 & 0.0001 & 15000 \\
\hline Iteration & Func-count & $\min f(x)$ & Procedure \\
\hline 0 & 4 & 38.3213 & \\
\hline 1 & 12 & 38.3213 & initial simplex \\
\hline 2 & 28 & 38.3213 & shrink \\
\hline 3 & 44 & 38.3213 & shrink \\
\hline 4 & 60 & 38.3213 & shrink \\
\hline 5 & 76 & 38.3213 & shrink \\
\hline 6 & 92 & 38.3213 & shrink \\
\hline 7 & 108 & 38.3213 & shrink \\
\hline 8 & 116 & 38.1922 & expand \\
\hline 9 & 120 & 38.1922 & reflect \\
\hline 10 & 128 & 38.0885 & expand \\
\hline 11 & 136 & 37.8421 & expand \\
\hline 12 & 144 & 37.5124 & expand \\
\hline 13 & 152 & 36.8687 & expand \\
\hline 14 & 160 & 35.9034 & expand \\
\hline 15 & 168 & 34.2218 & expand \\
\hline 16 & 176 & 31.6075 & expand \\
\hline 17 & 184 & 27.5019 & expand \\
\hline 18 & 192 & 21.4043 & expand \\
\hline 19 & 200 & 13.4983 & expand \\
\hline 20 & 208 & 10.1282 & reflect \\
\hline 21 & 224 & 10.1282 & shrink \\
\hline 22 & 232 & 9.4035 & reflect \\
\hline 23 & 248 & 9.37931 & shrink \\
\hline 24 & 264 & 9.25342 & shrink \\
\hline 25 & 268 & 9.25342 & reflect \\
\hline 26 & 276 & 9.25342 & contract \\
\hline 27 & 284 & 9.25342 & contract \\
\hline 28 & 292 & 9.25342 & contract \\
\hline 29 & 300 & 9.25342 & contract \\
\hline 30 & 308 & 9.25342 & contract \\
\hline 31 & 316 & 9.24775 & expand \\
\hline 32 & 320 & 9.24775 & reflect \\
\hline 33 & 328 & 9.24068 & expand \\
\hline 34 & 336 & 9.23092 & expand \\
\hline 35 & 344 & 9.21939 & expand \\
\hline 36 & 352 & 9.18595 & expand \\
\hline 37 & 360 & 9.17889 & expand \\
\hline 38 & 368 & 9.09544 & expand \\
\hline 39 & 372 & 9.09544 & reflect \\
\hline 40 & 380 & 8.99826 & expand \\
\hline 41 & 388 & 8.86286 & expand \\
\hline
\end{tabular}




\begin{tabular}{|c|c|c|c|}
\hline 42 & 396 & 8.64943 & expand \\
\hline 43 & 404 & 8.28432 & expand \\
\hline 44 & 412 & 7.88871 & expand \\
\hline 45 & 420 & 7.15808 & expand \\
\hline 46 & 428 & 6.66465 & expand \\
\hline 47 & 436 & 5.73331 & expand \\
\hline 48 & 444 & 5.39812 & expand \\
\hline 49 & 452 & 4.9825 & reflect \\
\hline 50 & 460 & 4.9825 & contract \\
\hline 51 & 468 & 4.72609 & expand \\
\hline 52 & 476 & 4.48985 & reflect \\
\hline 53 & 484 & 3.99258 & expand \\
\hline 54 & 492 & 3.5858 & expand \\
\hline 55 & 500 & 3.13197 & expand \\
\hline 56 & 508 & 2.50572 & expand \\
\hline 57 & 512 & 2.50572 & reflect \\
\hline 58 & 520 & 1.94473 & expand \\
\hline 59 & 528 & 1.94473 & contract \\
\hline 60 & 536 & 1.8023 & expand \\
\hline 61 & 552 & 1.78907 & shrink \\
\hline 62 & 560 & 1.64784 & reflect \\
\hline 63 & 568 & 1.64784 & contract \\
\hline 64 & 576 & 1.60724 & expand \\
\hline 65 & 592 & 1.60724 & shrink \\
\hline 66 & 596 & 1.60724 & reflect \\
\hline 67 & 604 & 1.59696 & reflect \\
\hline 68 & 612 & 1.59093 & contract \\
\hline 69 & 620 & 1.59093 & contract \\
\hline 70 & 628 & 1.59093 & contract \\
\hline 71 & 636 & 1.59093 & contract \\
\hline 72 & 644 & 1.59067 & contract \\
\hline 73 & 652 & 1.59061 & contract \\
\hline 74 & 660 & 1.59061 & contract \\
\hline 75 & 668 & 1.59058 & contract \\
\hline 76 & 676 & 1.59055 & contract \\
\hline 77 & 684 & 1.59055 & contract \\
\hline 78 & 688 & 1.59055 & reflect \\
\hline 79 & 696 & 1.59055 & contract \\
\hline 80 & 704 & 1.59055 & contract \\
\hline 81 & 712 & 1.59055 & contract \\
\hline 82 & 720 & 1.59055 & contract \\
\hline 83 & 728 & 1.59055 & contract \\
\hline 84 & 736 & 1.59055 & contract \\
\hline 85 & 744 & 1.59055 & contract \\
\hline 86 & 752 & 1.59055 & contract \\
\hline 87 & 760 & 1.59055 & contract \\
\hline 88 & 768 & 1.59055 & contract \\
\hline 89 & 776 & 1.59055 & contract \\
\hline 90 & 784 & 1.59055 & contract \\
\hline 91 & 792 & 1.59055 & contract \\
\hline 92 & 800 & 1.59055 & contract \\
\hline 93 & 808 & 1.59055 & contract \\
\hline 94 & 816 & 1.59055 & contract \\
\hline 95 & 824 & 1.59055 & contract \\
\hline
\end{tabular}




\begin{tabular}{|c|c|c|c|}
\hline 96 & 832 & 1.59055 & contract \\
\hline 97 & 840 & 1.59055 & contract \\
\hline 98 & 848 & 1.59055 & contract \\
\hline $\begin{array}{l}\text { Optimal KP } \\
0.712984\end{array}$ & $\begin{array}{c}\text { Optimal KI } \\
2.00618\end{array}$ & $\begin{array}{c}\text { Optimal cost J } \\
1.59055\end{array}$ & \\
\hline Algorithm run & 2 \# 3 & & \\
\hline $\begin{array}{l}\text { Starting KP } \\
0.712984\end{array}$ & $\begin{array}{l}\text { Starting KI } \\
2.00618\end{array}$ & $\begin{array}{c}\mathrm{mu} \\
0.0001\end{array}$ & $\begin{array}{c}\text { Duration } \mathrm{T} \text { (sec) } \\
500\end{array}$ \\
\hline Iteration & Func-count & $\min f(x)$ & Procedure \\
\hline 0 & 4 & 1.59055 & \\
\hline 1 & 12 & 1.59055 & initial simplex \\
\hline 2 & 16 & 1.59055 & reflect \\
\hline 3 & 24 & 1.59055 & contract \\
\hline 4 & 28 & 1.59055 & reflect \\
\hline 5 & 44 & 1.59055 & shrink \\
\hline 6 & 60 & 1.59055 & shrink \\
\hline 7 & 76 & 1.59055 & shrink \\
\hline 8 & 92 & 1.59055 & shrink \\
\hline 9 & 108 & 1.59055 & shrink \\
\hline 10 & 124 & 1.59055 & shrink \\
\hline 11 & 140 & 1.59055 & shrink \\
\hline 12 & 156 & 1.59055 & shrink \\
\hline 13 & 172 & 1.59055 & shrink \\
\hline 14 & 188 & 1.59055 & shrink \\
\hline 15 & 204 & 1.59055 & shrink \\
\hline 16 & 220 & 1.59055 & shrink \\
\hline 17 & 236 & 1.59055 & shrink \\
\hline 18 & 252 & 1.59055 & shrink \\
\hline 19 & 260 & 1.59055 & contract \\
\hline 20 & 264 & 1.59055 & reflect \\
\hline 21 & 272 & 1.59055 & reflect \\
\hline 22 & 280 & 1.59055 & contract \\
\hline 23 & 288 & 1.59055 & contract \\
\hline 24 & 296 & 1.59055 & contract \\
\hline $\begin{array}{l}\text { Optimal KP } \\
0.712985\end{array}$ & $\begin{array}{c}\text { Optimal KI } \\
2.00619\end{array}$ & $\begin{array}{c}\text { Optimal cost } \mathrm{J} \\
1.59055\end{array}$ & \\
\hline
\end{tabular}

$\underline{\text { Design } 2 \text { with } \theta=10}$ :

\begin{tabular}{|ccccc}
\hline $\begin{array}{c}\text { Algorithm run \# } \\
\text { Starting KP }\end{array}$ & $\begin{array}{c}\text { Starting } \\
\text { le-005 }\end{array}$ & theta & mu & Duration $\mathrm{T}$ (sec) \\
0.0005 & 10 & 0.0001 & 15000 \\
Iteration & Func-count & min $\mathrm{f}(\mathrm{x})$ & Procedure \\
0 & 4 & 486.743 & \\
1 & 12 & 485.683 & initial simplex \\
2 & 20 & 484.426 & expand
\end{tabular}




\begin{tabular}{|c|c|c|c|}
\hline 3 & 28 & 482.671 & expand \\
\hline 4 & 36 & 479.333 & expand \\
\hline 5 & 44 & 474.282 & expand \\
\hline 6 & 52 & 465.467 & expand \\
\hline 7 & 60 & 451.929 & expand \\
\hline 8 & 68 & 430.196 & expand \\
\hline 9 & 76 & 398.617 & expand \\
\hline 10 & 84 & 354.296 & expand \\
\hline 11 & 92 & 299.08 & expand \\
\hline 12 & 100 & 237.071 & expand \\
\hline 13 & 108 & 176.569 & expand \\
\hline 14 & 116 & 124.478 & expand \\
\hline 15 & 124 & 84.4005 & expand \\
\hline 16 & 132 & 56.2252 & expand \\
\hline 17 & 140 & 37.5146 & expand \\
\hline 18 & 148 & 31.9591 & reflect \\
\hline 19 & 164 & 31.9591 & shrink \\
\hline 20 & 180 & 31.9591 & shrink \\
\hline 21 & 188 & 30.1804 & reflect \\
\hline 22 & 196 & 29.3101 & reflect \\
\hline 23 & 212 & 29.3101 & shrink \\
\hline 24 & 228 & 29.3101 & shrink \\
\hline 25 & 244 & 29.3101 & shrink \\
\hline 26 & 248 & 29.3101 & reflect \\
\hline 27 & 256 & 29.3101 & contract \\
\hline 28 & 264 & 29.265 & contract \\
\hline 29 & 268 & 29.265 & reflect \\
\hline 30 & 284 & 29.265 & shrink \\
\hline 31 & 300 & 29.265 & shrink \\
\hline 32 & 308 & 29.2649 & reflect \\
\hline 33 & 316 & 29.2641 & reflect \\
\hline 34 & 324 & 29.2637 & contract \\
\hline 35 & 332 & 29.2635 & reflect \\
\hline 36 & 340 & 29.2635 & contract \\
\hline 37 & 348 & 29.2628 & expand \\
\hline 38 & 352 & 29.2628 & reflect \\
\hline 39 & 360 & 29.2615 & expand \\
\hline 40 & 364 & 29.2615 & reflect \\
\hline 41 & 372 & 29.261 & expand \\
\hline 42 & 380 & 29.258 & expand \\
\hline 43 & 384 & 29.258 & reflect \\
\hline 44 & 392 & 29.2519 & expand \\
\hline 45 & 396 & 29.2519 & reflect \\
\hline 46 & 404 & 29.2412 & expand \\
\hline 47 & 412 & 29.2343 & expand \\
\hline 48 & 420 & 29.2075 & expand \\
\hline 49 & 428 & 29.1872 & expand \\
\hline 50 & 436 & 29.1157 & expand \\
\hline 51 & 444 & 29.1008 & expand \\
\hline 52 & 452 & 28.9211 & expand \\
\hline 53 & 460 & 28.9211 & contract \\
\hline 54 & 468 & 28.774 & expand \\
\hline 55 & 476 & 28.4249 & expand \\
\hline 56 & 484 & 28.0376 & expand \\
\hline
\end{tabular}




\begin{tabular}{|c|c|c|c|}
\hline 57 & 492 & 27.1478 & expand \\
\hline 58 & 500 & 26.1245 & expand \\
\hline 59 & 508 & 24.1048 & expand \\
\hline 60 & 516 & 21.6709 & expand \\
\hline 61 & 524 & 17.8847 & expand \\
\hline 62 & 532 & 13.6857 & expand \\
\hline 63 & 540 & 8.92275 & expand \\
\hline 64 & 548 & 4.82966 & expand \\
\hline 65 & 556 & 1.96297 & expand \\
\hline 66 & 564 & 1.32873 & expand \\
\hline 67 & 572 & 1.32873 & contract \\
\hline 68 & 580 & 1.25181 & contract \\
\hline 69 & 584 & 1.25181 & reflect \\
\hline 70 & 592 & 1.2166 & contract \\
\hline 71 & 596 & 1.2166 & reflect \\
\hline 72 & 604 & 1.21398 & contract \\
\hline 73 & 612 & 1.21398 & contract \\
\hline 74 & 620 & 1.21312 & reflect \\
\hline 75 & 628 & 1.2107 & contract \\
\hline 76 & 636 & 1.20909 & expand \\
\hline 77 & 644 & 1.20909 & contract \\
\hline 78 & 652 & 1.20613 & expand \\
\hline 79 & 656 & 1.20613 & reflect \\
\hline 80 & 664 & 1.20096 & expand \\
\hline 81 & 668 & 1.20096 & reflect \\
\hline 82 & 676 & 1.19688 & expand \\
\hline 83 & 684 & 1.19642 & reflect \\
\hline 84 & 700 & 1.19617 & shrink \\
\hline 85 & 708 & 1.19617 & contract \\
\hline 86 & 716 & 1.19617 & contract \\
\hline 87 & 724 & 1.19614 & contract \\
\hline 88 & 732 & 1.19612 & contract \\
\hline 89 & 740 & 1.19612 & contract \\
\hline 90 & 748 & 1.19612 & contract \\
\hline 91 & 756 & 1.19611 & contract \\
\hline 92 & 764 & 1.19611 & contract \\
\hline 93 & 772 & 1.19611 & contract \\
\hline 94 & 776 & 1.19611 & reflect \\
\hline 95 & 784 & 1.19611 & contract \\
\hline 96 & 792 & 1.19611 & contract \\
\hline 97 & 800 & 1.19611 & contract \\
\hline 98 & 808 & 1.19611 & contract \\
\hline 99 & 816 & 1.19611 & contract \\
\hline 100 & 824 & 1.19611 & contract \\
\hline 101 & 832 & 1.19611 & contract \\
\hline 102 & 840 & 1.19611 & contract \\
\hline 103 & 848 & 1.19611 & contract \\
\hline 104 & 856 & 1.19611 & contract \\
\hline 105 & 860 & 1.19611 & reflect \\
\hline 106 & 868 & 1.19611 & contract \\
\hline 107 & 876 & 1.19611 & contract \\
\hline $\begin{array}{l}\text { ptimal KP } \\
0.451414\end{array}$ & $\begin{array}{c}\text { Optimal KI } \\
0.137636\end{array}$ & $\begin{array}{c}\text { Optimal cost } \mathrm{J} \\
1.19611\end{array}$ & \\
\hline
\end{tabular}




\begin{tabular}{|c|c|c|c|c|}
\hline $\begin{array}{l}\text { Starting KP } \\
0.451414\end{array}$ & $\begin{array}{l}\text { Starting KI } \\
0.137636\end{array}$ & $\begin{array}{c}\text { theta } \\
10\end{array}$ & $\begin{array}{c}\mathrm{mu} \\
0.0001\end{array}$ & $\begin{array}{c}\text { Duration } \\
300\end{array}$ \\
\hline Iteration & Func-count & $\min f(x)$ & & Procedure \\
\hline 0 & 4 & 1.19611 & & \\
\hline 1 & 12 & 1.19611 & & initial simplex \\
\hline 2 & 20 & 1.19611 & & contract \\
\hline 3 & 28 & 1.19611 & & contract \\
\hline 4 & 36 & 1.19611 & & contract \\
\hline 5 & 40 & 1.19611 & & reflect \\
\hline 6 & 48 & 1.19611 & & contract \\
\hline 7 & 56 & 1.19611 & & contract \\
\hline 8 & 64 & 1.19611 & & contract \\
\hline 9 & 68 & 1.19611 & & reflect \\
\hline 10 & 76 & 1.19611 & & contract \\
\hline 11 & 84 & 1.19611 & & contract \\
\hline 12 & 92 & 1.19611 & & contract \\
\hline 13 & 100 & 1.19611 & & contract \\
\hline 14 & 108 & 1.19611 & & contract \\
\hline 15 & 116 & 1.19611 & & contract \\
\hline 16 & 124 & 1.19611 & & contract \\
\hline 17 & 132 & 1.19611 & & contract \\
\hline 18 & 140 & 1.19611 & & contract \\
\hline 19 & 148 & 1.19611 & & contract \\
\hline 20 & 156 & 1.19611 & & contract \\
\hline 21 & 164 & 1.19611 & & contract \\
\hline 22 & 172 & 1.19611 & & contract \\
\hline 23 & 180 & 1.19611 & & contract \\
\hline 24 & 188 & 1.19611 & & contract \\
\hline 25 & 196 & 1.19611 & & contract \\
\hline 26 & 204 & 1.19611 & & contract \\
\hline 27 & 212 & 1.19611 & & contract \\
\hline 28 & 216 & 1.19611 & & reflect \\
\hline 29 & 224 & 1.19611 & & contract \\
\hline 30 & 232 & 1.19611 & & contract \\
\hline 31 & 240 & 1.19611 & & contract \\
\hline Optimal KP & Optimal KI & Optimal & cost $\mathrm{J}$ & \\
\hline 0.451414 & 0.137636 & 1.1 & 9611 & \\
\hline
\end{tabular}




\section{C.3 Control Design for Time-Delayed System}

The following optimization output was obtained when designing the two-term controller (7.6) for the SISO time-delayed system in Section 7.4.

Design of Controller for the Case $t_{0}=0 s$ :

\begin{tabular}{|c|c|c|c|c|}
\hline \multicolumn{5}{|c|}{ Algorithm run \# 1} \\
\hline $\begin{array}{c}\text { Starting KP } \\
0.005\end{array}$ & $\begin{array}{c}\text { Starting KI } \\
0.001\end{array}$ & $\begin{array}{c}\text { theta } \\
0\end{array}$ & $\begin{array}{c}\mathrm{mu} \\
1 e^{-008}\end{array}$ & $\begin{array}{l}\text { Duration } \mathrm{T} \text { (sec) } \\
\quad 10000\end{array}$ \\
\hline Iteration & Func-count & $\min \mathrm{f}(\mathrm{x})$ & & Procedure \\
\hline 0 & 1 & 510.057 & & \\
\hline 1 & 3 & 419.381 & & initial simplex \\
\hline 2 & 5 & 343.394 & & expand \\
\hline 3 & 7 & 279.258 & & expand \\
\hline 4 & 9 & 202.944 & & expand \\
\hline 5 & 11 & 146.48 & & expand \\
\hline 6 & 13 & 98.432 & & expand \\
\hline 7 & 15 & 67.3507 & & expand \\
\hline 8 & 17 & 45.5836 & & expand \\
\hline 9 & 19 & 32.6306 & & expand \\
\hline 10 & 21 & 24.5119 & & expand \\
\hline 11 & 23 & 20.3691 & & expand \\
\hline 12 & 25 & 18.8239 & & expand \\
\hline 13 & 26 & 18.8239 & & reflect \\
\hline 14 & 28 & 18.8239 & & contract \\
\hline 15 & 30 & 18.8239 & & contract \\
\hline 16 & 32 & 18.8239 & & contract \\
\hline 17 & 34 & 18.8239 & & contract \\
\hline 18 & 36 & 18.7215 & & expand \\
\hline 19 & 37 & 18.7215 & & reflect \\
\hline 20 & 39 & 18.5328 & & expand \\
\hline 21 & 41 & 18.4331 & & expand \\
\hline 22 & 43 & 17.9814 & & expand \\
\hline 23 & 45 & 17.7027 & & expand \\
\hline 24 & 47 & 16.6433 & & expand \\
\hline 25 & 49 & 16.2956 & & expand \\
\hline 26 & 51 & 14.1993 & & expand \\
\hline 27 & 52 & 14.1993 & & reflect \\
\hline 28 & 54 & 12.1035 & & expand \\
\hline 29 & 56 & 10.6963 & & expand \\
\hline 30 & 58 & 8.43731 & & expand \\
\hline 31 & 60 & 8.15482 & & expand \\
\hline 32 & 62 & 7.99759 & & reflect \\
\hline 33 & 64 & 7.79621 & & contract \\
\hline 34 & 66 & 7.56982 & & reflect \\
\hline 35 & 68 & 7.56982 & & contract \\
\hline 36 & 70 & 7.56982 & & contract \\
\hline 37 & 72 & 7.54407 & & reflect \\
\hline 38 & 74 & 7.54407 & & contract \\
\hline
\end{tabular}




\begin{tabular}{|c|c|c|c|}
\hline 39 & 76 & 7.538 & contract \\
\hline 40 & 78 & 7.53593 & contract \\
\hline 41 & 80 & 7.53265 & contract \\
\hline 42 & 82 & 7.53265 & contract \\
\hline 43 & 83 & 7.53265 & reflect \\
\hline 44 & 85 & 7.53176 & contract \\
\hline 45 & 87 & 7.53176 & contract \\
\hline 46 & 89 & 7.53176 & contract \\
\hline 47 & 91 & 7.53174 & contract \\
\hline 48 & 93 & 7.53172 & contract \\
\hline 49 & 95 & 7.53167 & contract \\
\hline 50 & 96 & 7.53167 & reflect \\
\hline 51 & 98 & 7.53167 & contract \\
\hline 52 & 99 & 7.53167 & reflect \\
\hline 53 & 100 & 7.53167 & reflect \\
\hline 54 & 102 & 7.53167 & contract \\
\hline 55 & 104 & 7.53167 & contract \\
\hline 56 & 106 & 7.53167 & contract \\
\hline 57 & 108 & 7.53166 & contract \\
\hline 58 & 110 & 7.53166 & contract \\
\hline 59 & 112 & 7.53166 & reflect \\
\hline 60 & 114 & 7.53166 & contract \\
\hline 61 & 116 & 7.53166 & contract \\
\hline 62 & 118 & 7.53166 & contract \\
\hline 63 & 120 & 7.53166 & contract \\
\hline 64 & 122 & 7.53166 & contract \\
\hline 65 & 124 & 7.53166 & contract \\
\hline 66 & 126 & 7.53166 & contract \\
\hline $\begin{array}{c}\text { Optimal KP } \\
3.25181\end{array}$ & $\begin{array}{l}\text { Optimal KI } \\
0.105815\end{array}$ & $\begin{array}{c}\text { Optimal cost } \mathrm{J} \\
7.53166\end{array}$ & \\
\hline Algorithm run & 1 \# 2 & & \\
\hline Starting $\mathrm{KP}$ & Starting KI & theta & Duration $\mathrm{T}$ (sec) \\
\hline 3.25181 & 0.105815 & $1 e-008$ & 10000 \\
\hline Iteration & Func-count & $\min f(x)$ & Procedure \\
\hline 0 & 1 & 7.53166 & \\
\hline 1 & 3 & 7.53166 & initial simplex \\
\hline 2 & 4 & 7.53166 & reflect \\
\hline 3 & 6 & 7.53166 & contract \\
\hline 4 & 8 & 7.53166 & contract \\
\hline 5 & 10 & 7.53166 & contract \\
\hline 6 & 12 & 7.53166 & contract \\
\hline 7 & 14 & 7.53166 & contract \\
\hline 8 & 16 & 7.53166 & contract \\
\hline 9 & 18 & 7.53166 & contract \\
\hline 10 & 20 & 7.53166 & contract \\
\hline 11 & 22 & 7.53166 & contract \\
\hline 12 & 24 & 7.53166 & contract \\
\hline 13 & 26 & 7.53166 & contract \\
\hline 14 & 28 & 7.53166 & contract \\
\hline 15 & 30 & 7.53166 & contract \\
\hline
\end{tabular}




\begin{tabular}{|llll|}
16 & 32 & 7.53166 & contract \\
17 & 34 & 7.53166 & contract \\
18 & 36 & 7.53166 & contract \\
19 & 38 & 7.53166 & contract \\
20 & 40 & 7.53166 & contract \\
21 & 42 & 7.53166 & contract \\
22 & 44 & 7.53166 & contract \\
23 & 46 & 7.53166 & contract \\
24 & 47 & 7.53166 & reflect \\
25 & 49 & 7.53166 & contract \\
26 & 50 & 7.53166 & reflect \\
27 & 52 & 7.53166 & contract \\
28 & 56 & 7.53166 & \\
Optimal KP & Optimal KI & Optimal cost J J & \\
3.25188 & 0.105814 & 7.53166 & \\
\hline
\end{tabular}

Design of Controller for the Case $t_{0}=10 \mathrm{~s}$ :

\begin{tabular}{|c|c|c|c|}
\hline \multicolumn{4}{|c|}{ Algorithm run \# 1} \\
\hline $\begin{array}{c}\text { Starting KP } \\
0.005\end{array}$ & $\begin{array}{l}\text { Starting KI } \\
0.001\end{array}$ & $\begin{array}{c}\text { theta } \\
0\end{array}$ & $\begin{array}{l}\text { Duration } \mathrm{T} \text { (sec) } \\
\quad 10000\end{array}$ \\
\hline Iteration & Func-count & $\min f(x)$ & Procedure \\
\hline 0 & 1 & 500.065 & \\
\hline 1 & 3 & 409.39 & initial simplex \\
\hline 2 & 5 & 333.405 & expand \\
\hline 3 & 7 & 269.27 & expand \\
\hline 4 & 9 & 192.961 & expand \\
\hline 5 & 11 & 136.501 & expand \\
\hline 6 & 13 & 88.4665 & expand \\
\hline 7 & 15 & 57.3994 & expand \\
\hline 8 & 17 & 35.6658 & expand \\
\hline 9 & 19 & 22.7576 & expand \\
\hline 10 & 21 & 14.7269 & expand \\
\hline 11 & 23 & 10.7147 & expand \\
\hline 12 & 25 & 9.40087 & expand \\
\hline 13 & 26 & 9.40087 & reflect \\
\hline 14 & 28 & 9.40087 & contract \\
\hline 15 & 32 & 9.40087 & shrink \\
\hline 16 & 34 & 9.40087 & contract \\
\hline 17 & 36 & 9.31978 & expand \\
\hline 18 & 38 & 9.31978 & contract \\
\hline 19 & 40 & 9.31395 & expand \\
\hline 20 & 42 & 9.16123 & expand \\
\hline 21 & 43 & 9.16123 & reflect \\
\hline 22 & 45 & 9.08315 & expand \\
\hline 23 & 47 & 8.74462 & expand \\
\hline 24 & 49 & 8.43401 & expand \\
\hline 25 & 51 & 7.67938 & expand \\
\hline 26 & 53 & 6.826 & expand \\
\hline 27 & 55 & 5.40933 & expand \\
\hline
\end{tabular}




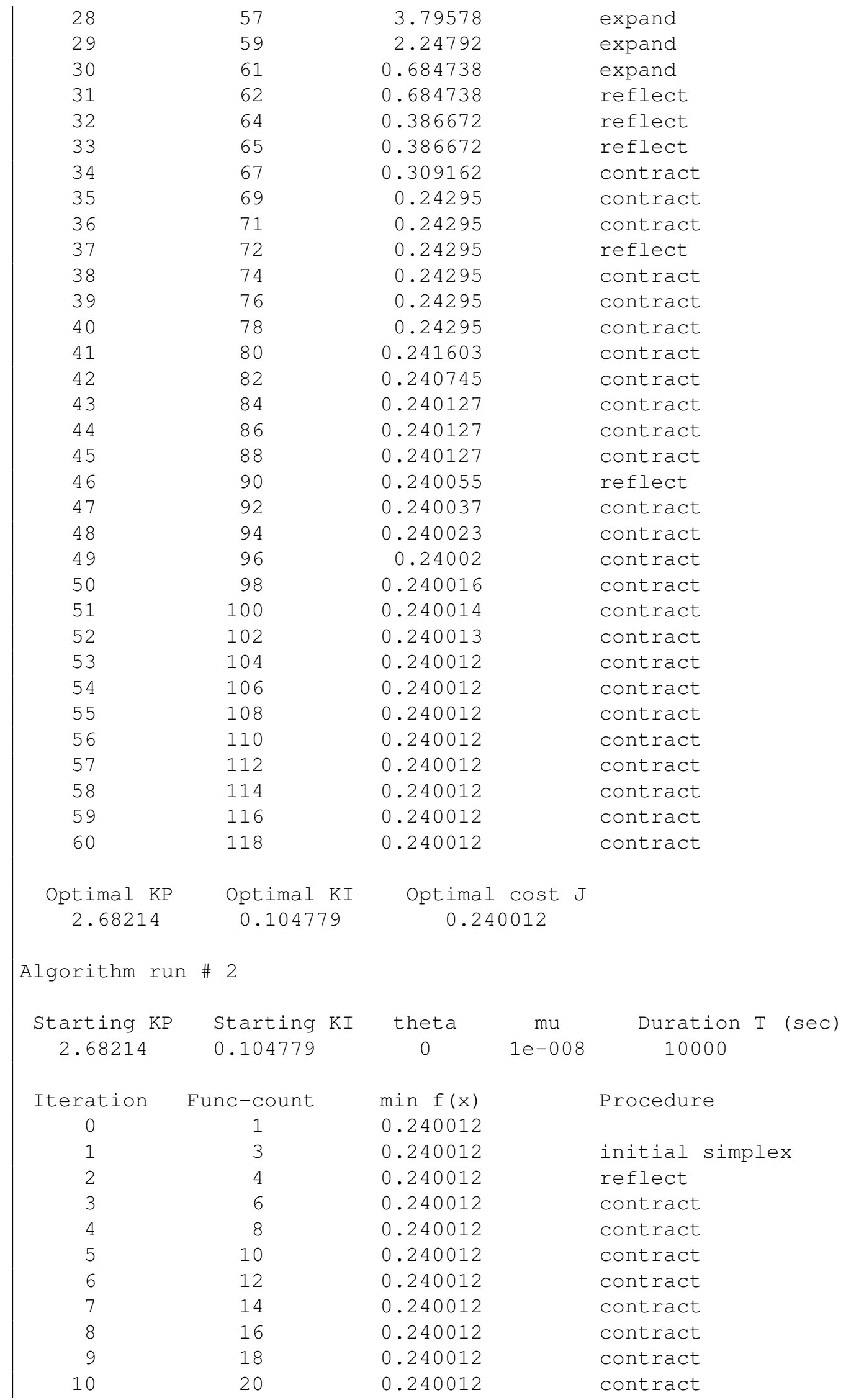




\begin{tabular}{|llll|}
11 & 22 & 0.240012 & contract \\
12 & 24 & 0.240012 & contract \\
13 & 26 & 0.240012 & contract \\
14 & 28 & 0.240012 & contract \\
15 & 30 & 0.240012 & contract \\
16 & 32 & 0.240012 & contract \\
17 & 34 & 0.240012 & contract \\
18 & 36 & 0.240012 & contract \\
19 & 38 & 0.240012 & contract \\
20 & 40 & 0.240012 & contract \\
21 & 41 & 0.240012 & reflect \\
22 & 43 & 0.240012 & contract \\
23 & 45 & 0.240012 & contract \\
24 & 47 & 0.240012 & contract \\
25 & 48 & 0.240012 & reflect \\
26 & 50 & 0.240012 & contract \\
27 & 52 & 0.240012 & \\
Optimal KP & Optimal KI & Optimal cost & J \\
2.68205 & 0.104782 & 0.240012 & \\
\hline
\end{tabular}

Design of Controller for the Case $t_{0}=18 \mathrm{~s}$ :

\begin{tabular}{|c|c|c|c|c|}
\hline \multicolumn{5}{|c|}{ Algorithm run \# 1} \\
\hline $\begin{array}{c}\text { Starting KP } \\
0.0001\end{array}$ & $\begin{array}{c}\text { Starting KI } \\
0.001\end{array}$ & $\begin{array}{c}\text { theta } \\
0\end{array}$ & $\begin{array}{c}\mathrm{mu} \\
1 \mathrm{e}-008\end{array}$ & $\begin{array}{l}\text { Duration } \mathrm{T} \text { (sec) } \\
\quad 10000\end{array}$ \\
\hline Iteration & Func-count & $\min f(x)$ & & Procedure \\
\hline 0 & 1 & 494.588 & & \\
\hline 1 & 3 & 403.703 & & initial simplex \\
\hline 2 & 5 & 327.965 & & expand \\
\hline 3 & 7 & 263.331 & & expand \\
\hline 4 & 9 & 187.25 & & expand \\
\hline 5 & 11 & 130.436 & & expand \\
\hline 6 & 13 & 82.666 & & expand \\
\hline 7 & 15 & 51.5358 & & expand \\
\hline 8 & 17 & 30.2733 & & expand \\
\hline 9 & 19 & 17.8395 & & expand \\
\hline 10 & 21 & 11.1073 & & expand \\
\hline 11 & 23 & 9.50261 & & expand \\
\hline 12 & 24 & 9.50261 & & reflect \\
\hline 13 & 26 & 9.42662 & & contract \\
\hline 14 & 30 & 9.4024 & & shrink \\
\hline 15 & 34 & 9.39781 & & shrink \\
\hline 16 & 38 & 9.39609 & & shrink \\
\hline 17 & 42 & 9.39592 & & shrink \\
\hline 18 & 46 & 9.39575 & & shrink \\
\hline 19 & 48 & 9.39575 & & contract \\
\hline 20 & 50 & 9.39573 & & contract \\
\hline 21 & 52 & 9.39573 & & contract \\
\hline 22 & 54 & 9.39564 & & expand \\
\hline 23 & 56 & 9.39564 & & contract \\
\hline
\end{tabular}




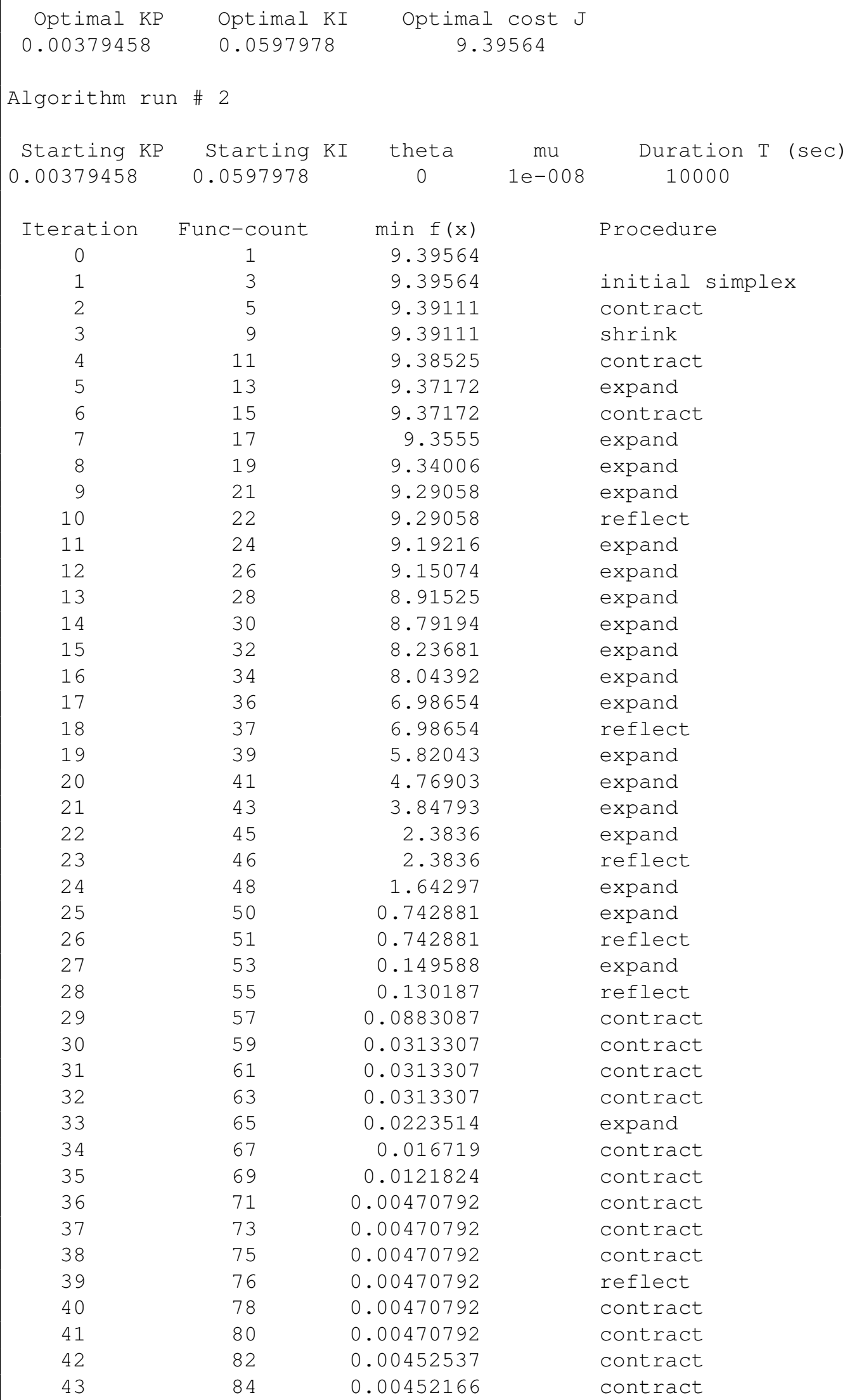




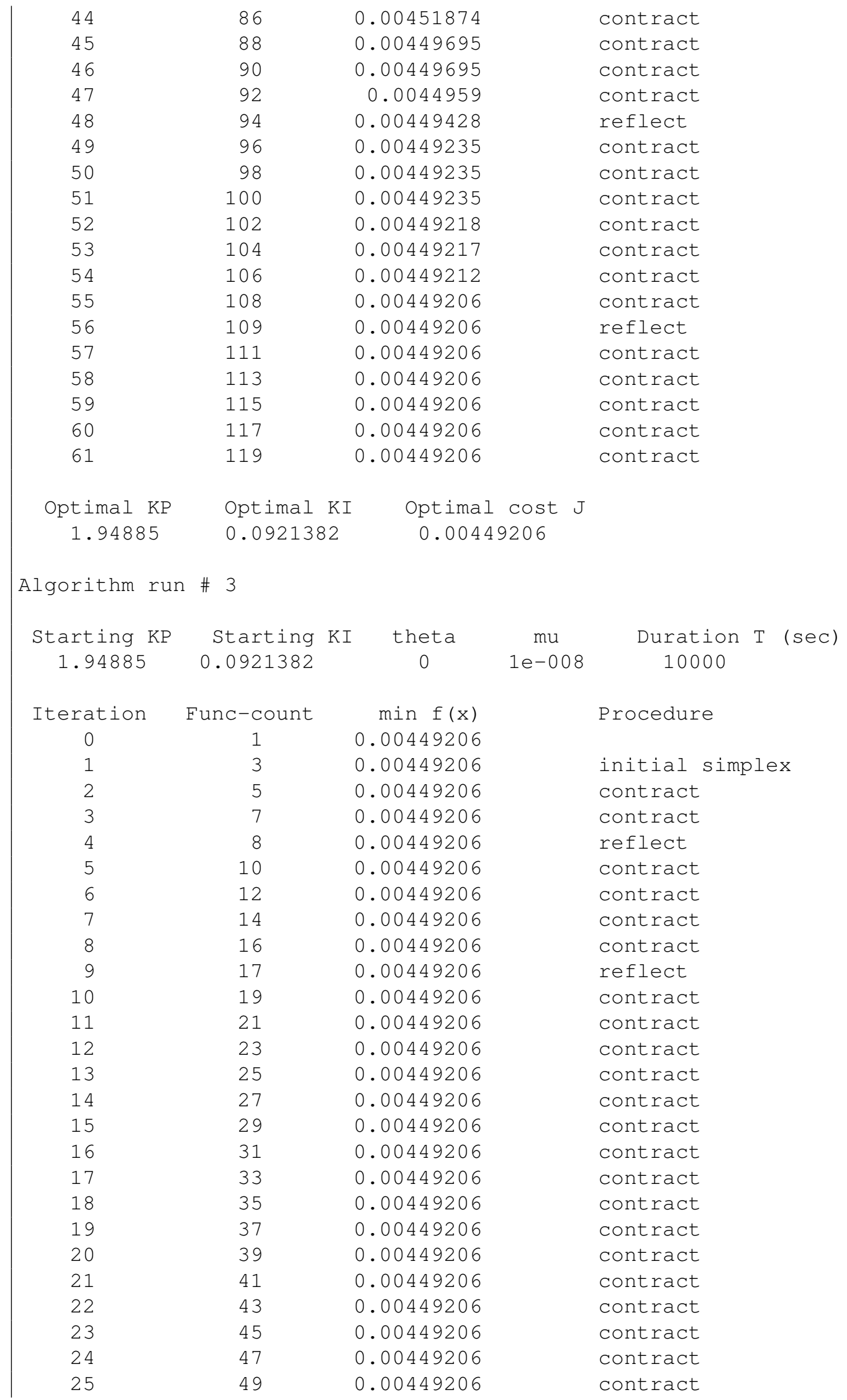




$\begin{array}{cccc}26 & 51 & 0.00449206 & \text { contract } \\ 27 & 53 & 0.00449206 & \text { contract } \\ 28 & 54 & 0.00449206 & \text { reflect } \\ & & & \\ \text { Optimal KP } & \text { Optimal KI } & \text { Optimal cost J } & \\ 1.94885 & 0.0921382 & 0.00449206 & \\ & & & \end{array}$

\section{C.4 Control Design for Experimental MARTS}

The following optimization output was obtained when designing the two-term controller (2.6) for the experimental MARTS system in Section 8.5.

\begin{tabular}{|c|c|c|c|c|c|}
\hline \multicolumn{6}{|c|}{ Algorithm run \# 1} \\
\hline $\begin{array}{c}\text { Starting KP } \\
0.01\end{array}$ & $\begin{array}{r}\text { Starting } \\
0.005\end{array}$ & $\mathrm{KI}$ & $\begin{array}{c}\text { theta } \\
0\end{array}$ & $\begin{array}{l}\mathrm{mu} \\
1\end{array}$ & $\begin{array}{l}\text { Duration } \mathrm{T} \text { (sec) } \\
500\end{array}$ \\
\hline Iteration & Func-count & & $\min \mathrm{f}(\mathrm{x})$ & & Procedure \\
\hline 0 & 1 & & 1068.18 & & \\
\hline 1 & 3 & & 246.505 & & initial simplex \\
\hline 2 & 5 & & 123.664 & & expand \\
\hline 3 & 7 & & 87.3302 & & expand \\
\hline 4 & 9 & & 79.3822 & & expand \\
\hline 5 & 11 & & 79.3822 & & contract \\
\hline 6 & 13 & & 79.3822 & & contract \\
\hline 7 & 15 & & 79.1632 & & reflect \\
\hline 8 & 19 & & 78.0586 & & shrink \\
\hline 9 & 21 & & 76.8838 & & expand \\
\hline 10 & 23 & & 76.8838 & & contract \\
\hline 11 & 25 & & 73.5444 & & expand \\
\hline 12 & 27 & & 72.0638 & & expand \\
\hline 13 & 29 & & 70.1961 & & expand \\
\hline 14 & 30 & & 70.1961 & & reflect \\
\hline 15 & 32 & & 68.231 & & expand \\
\hline 16 & 34 & & 65.8539 & & expand \\
\hline 17 & 35 & & 65.8539 & & reflect \\
\hline 18 & 36 & & 65.8539 & & reflect \\
\hline 19 & 38 & & 64.1645 & & contract \\
\hline 20 & 40 & & 64.1645 & & contract \\
\hline 21 & 42 & & 64.1607 & & reflect \\
\hline 22 & 44 & & 63.7266 & & contract \\
\hline 23 & 48 & & 63.7266 & & shrink \\
\hline 24 & 50 & & 63.282 & & reflect \\
\hline 25 & 52 & & 63.282 & & contract \\
\hline 26 & 56 & & 63.282 & & shrink \\
\hline 27 & 58 & & 63.282 & & contract \\
\hline Optimal KP & Optimal & $\mathrm{KI}$ & Optimal & st $J$ & \\
\hline
\end{tabular}




\begin{tabular}{|c|c|c|c|}
\hline \multicolumn{4}{|c|}{ Algorithm run \# 2} \\
\hline $\begin{array}{l}\text { Starting KP } \\
0.642618\end{array}$ & $\begin{array}{l}\text { Starting KI } \\
0.0775545\end{array}$ & $\begin{array}{c}\text { theta } \\
0\end{array}$ & $\begin{array}{l}\text { Duration } \mathrm{T} \text { (sec) } \\
240\end{array}$ \\
\hline Iteration & Func-count & $\min f(x)$ & Procedure \\
\hline 0 & 1 & 61.0768 & \\
\hline 1 & 3 & 61.0768 & initial simplex \\
\hline 2 & 5 & 61.0768 & contract \\
\hline 3 & 7 & 61.0768 & contract \\
\hline 4 & 8 & 61.0768 & reflect \\
\hline 5 & 10 & 61.0768 & contract \\
\hline 6 & 12 & 61.0768 & contract \\
\hline 7 & 13 & 61.0768 & reflect \\
\hline 8 & 15 & 61.0768 & contract \\
\hline 9 & 17 & 61.0768 & contract \\
\hline 10 & 19 & 61.0768 & contract \\
\hline 11 & 23 & 61.0768 & shrink \\
\hline 12 & 27 & 61.0768 & shrink \\
\hline 13 & 29 & 61.0768 & contract \\
\hline 14 & 30 & 61.0768 & reflect \\
\hline 15 & 34 & 61.0768 & shrink \\
\hline 16 & 38 & 61.0768 & shrink \\
\hline Optimal KP & Optimal KI & Optimal cost $\mathrm{J}$ & \\
\hline 0.642618 & 0.0775545 & 61.0768 & \\
\hline
\end{tabular}




\section{Appendix D}

\section{Program Listings}

This appendix contains MATLAB programs and Simulink models that were used to generate the simulation and experimental results presented in the thesis document.

\section{D.1 Valve Linearization}

The following two programs were used to obtain the square-root functions for MARTS control valve linearization.

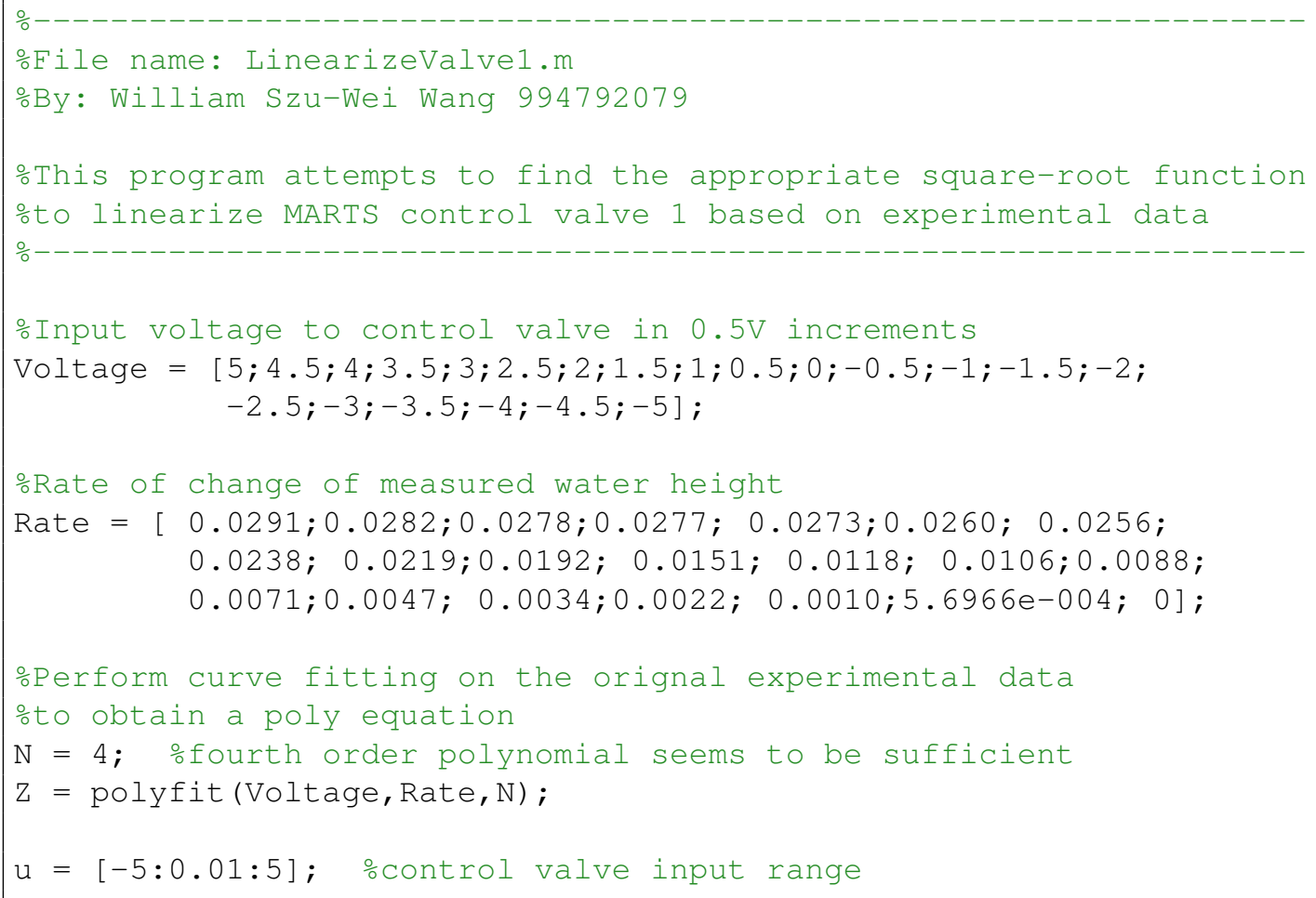


for alpha=0.1:0.1:3 oplot a curve for each alpha

new_u_to_output $=(($ alpha*sqrt $((u+5)))-5)$; \%apply square root fcn

$\mathrm{h}=$ polyval ( $\mathrm{z}, \mathrm{new} \mathrm{u}_{-}$o_output); \%obtain corresponding rates

plot $(u, h)$, hold on;

end

plot(Voltage, Rate, 'r'); \%impose the experimental data

hold off;

ylabel ('Rate of water height increase in Vessel 1 (V/S)')

xlabel ('Control input voltage (V)')

oNote: the plot shows that no alpha value gives rise to a better

olinear characteristic than the experimental data itself without

osacrificing maximum flow rate.

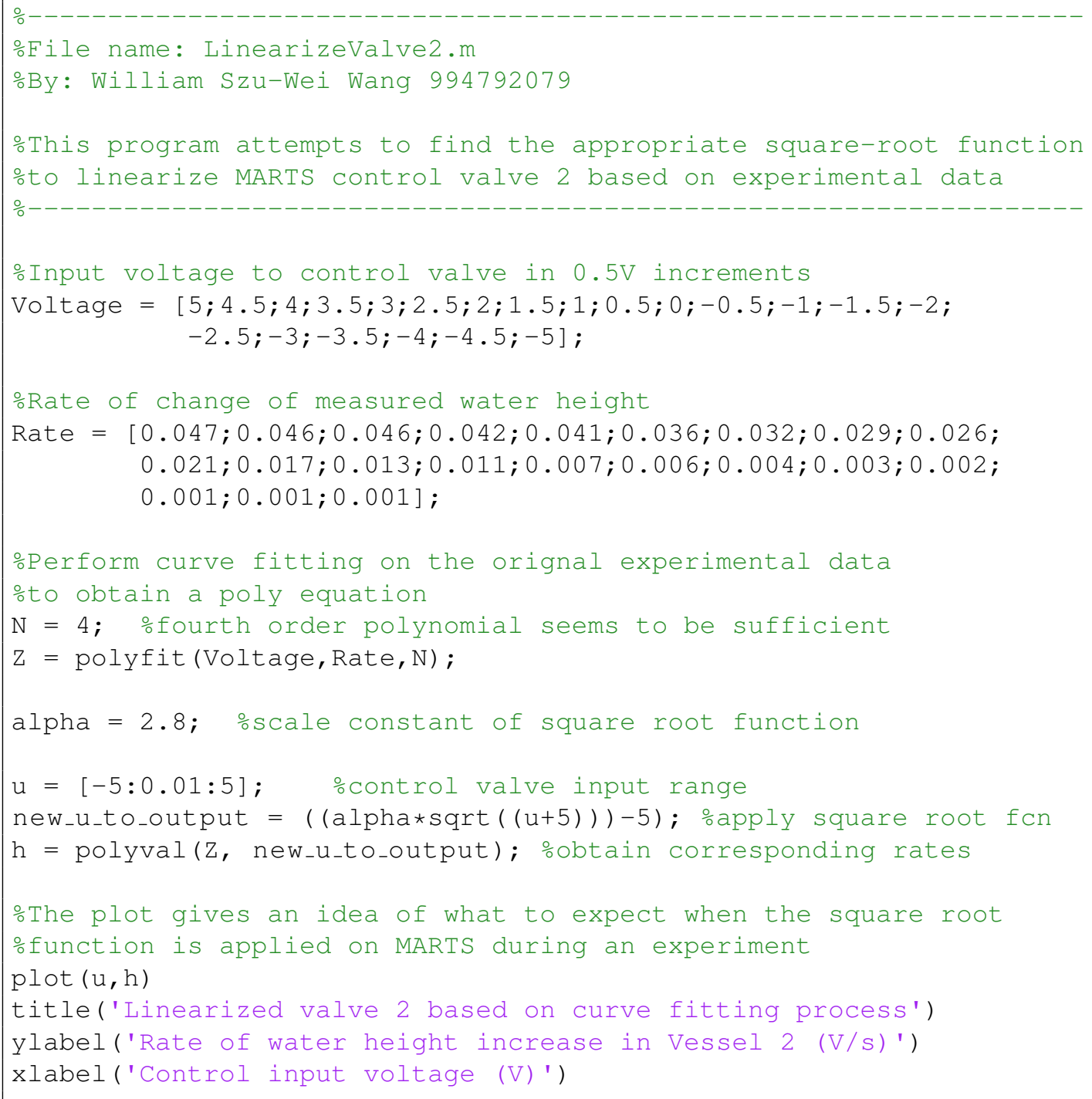




\section{D.2 Proportional Controller Design}

The following program was used to design the proportional controller in Section 3.2.

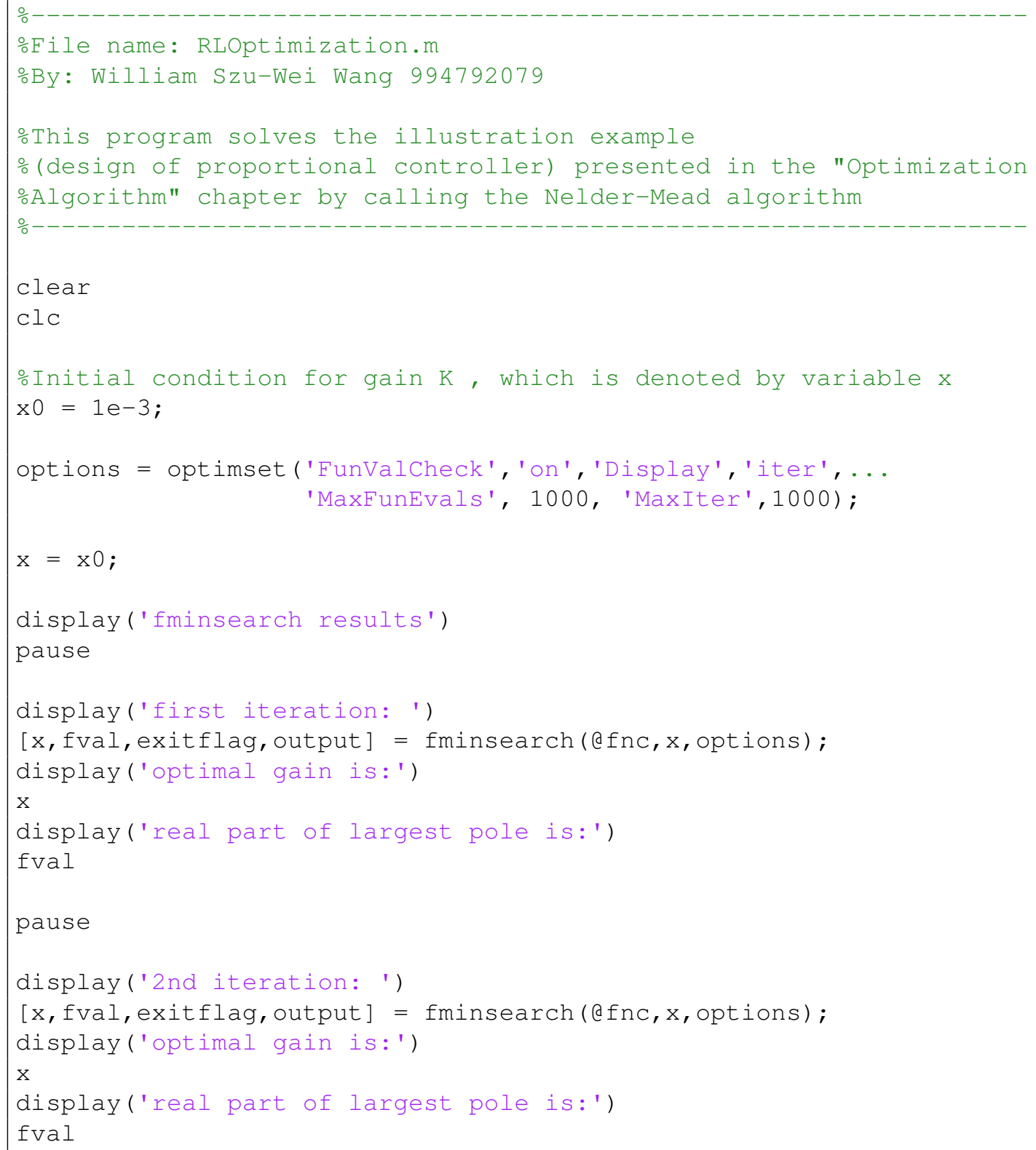




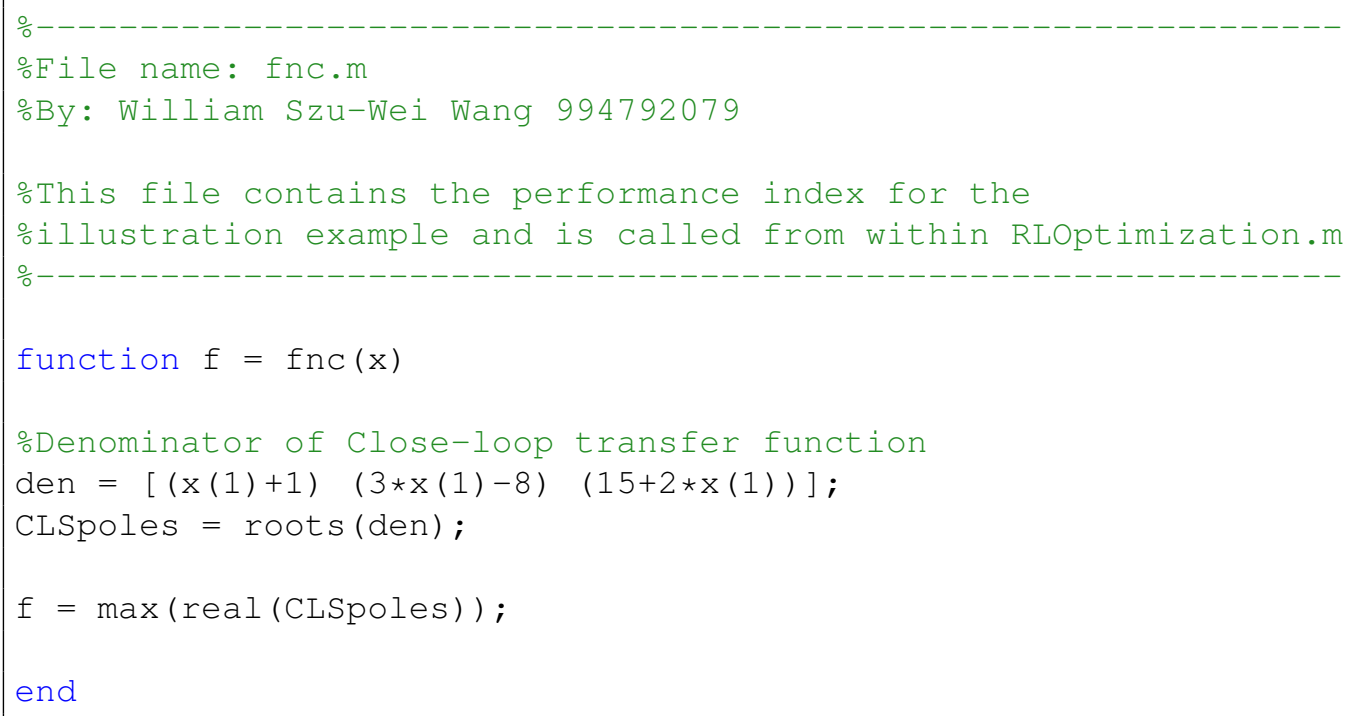

\section{D.3 Extremum Seeking}

The following program and Simulink model were used to carry out the extremum seeking controller design example in Section 7.

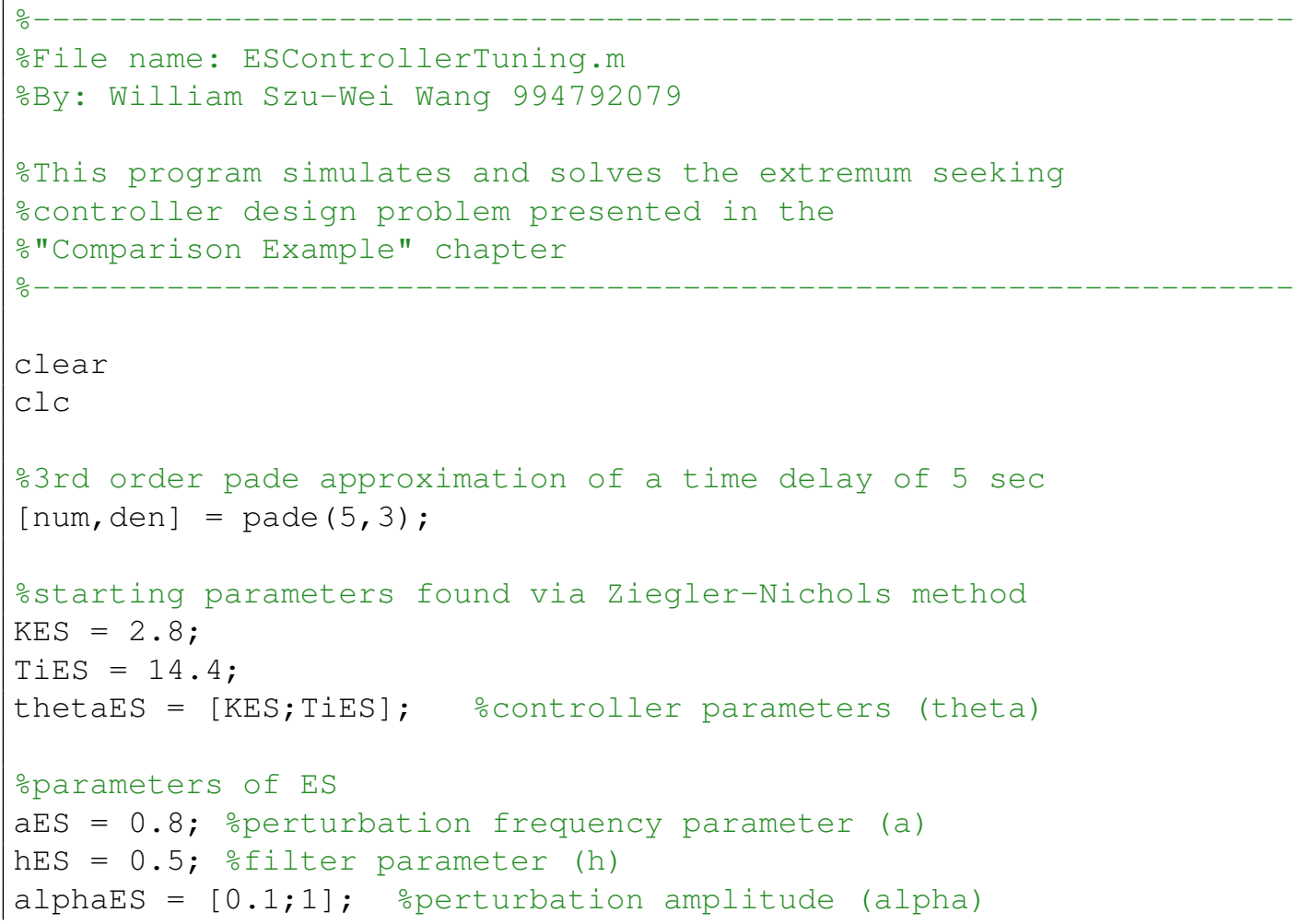




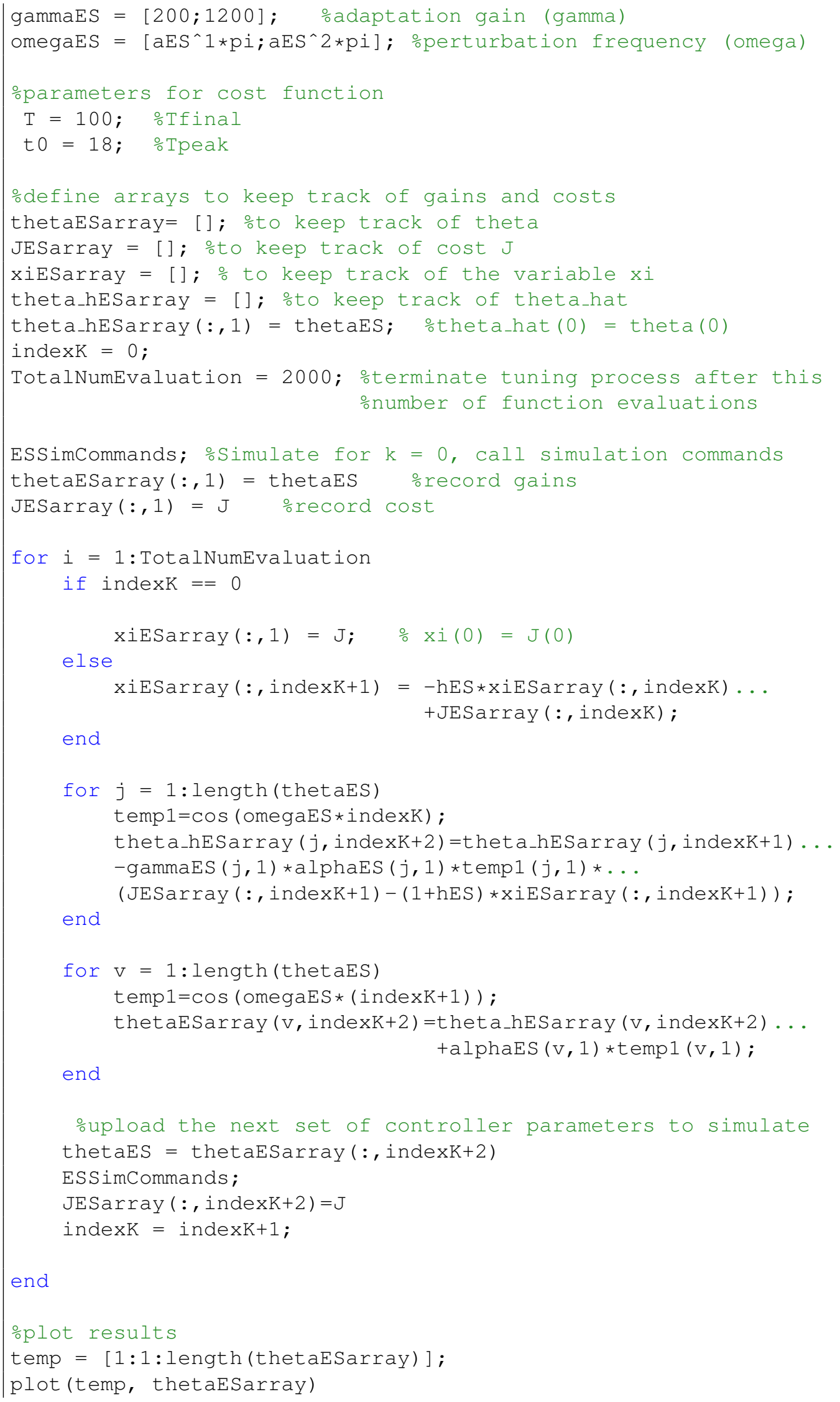


xlabel ('Number of function evaluations, k')

ylabel ('Controller parameters \theta')

legend ('K', ' $T_{-} i$ ')

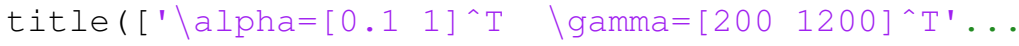

' lomega $=\left[\begin{array}{lll}0.8 \backslash p i & 0.64 \backslash p i{ }^{\wedge} \mathrm{T} & \text { ' }])\end{array}\right.$

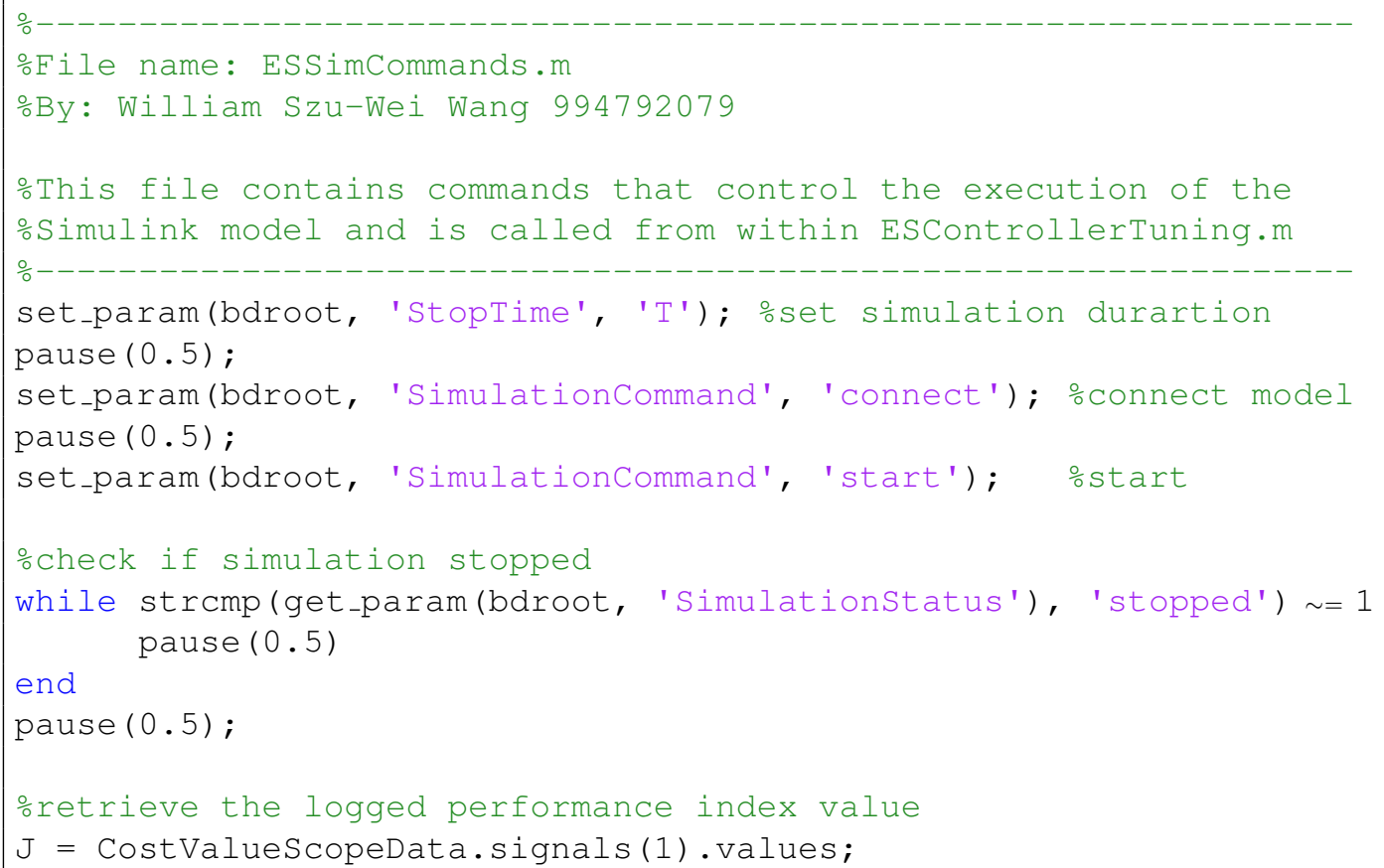




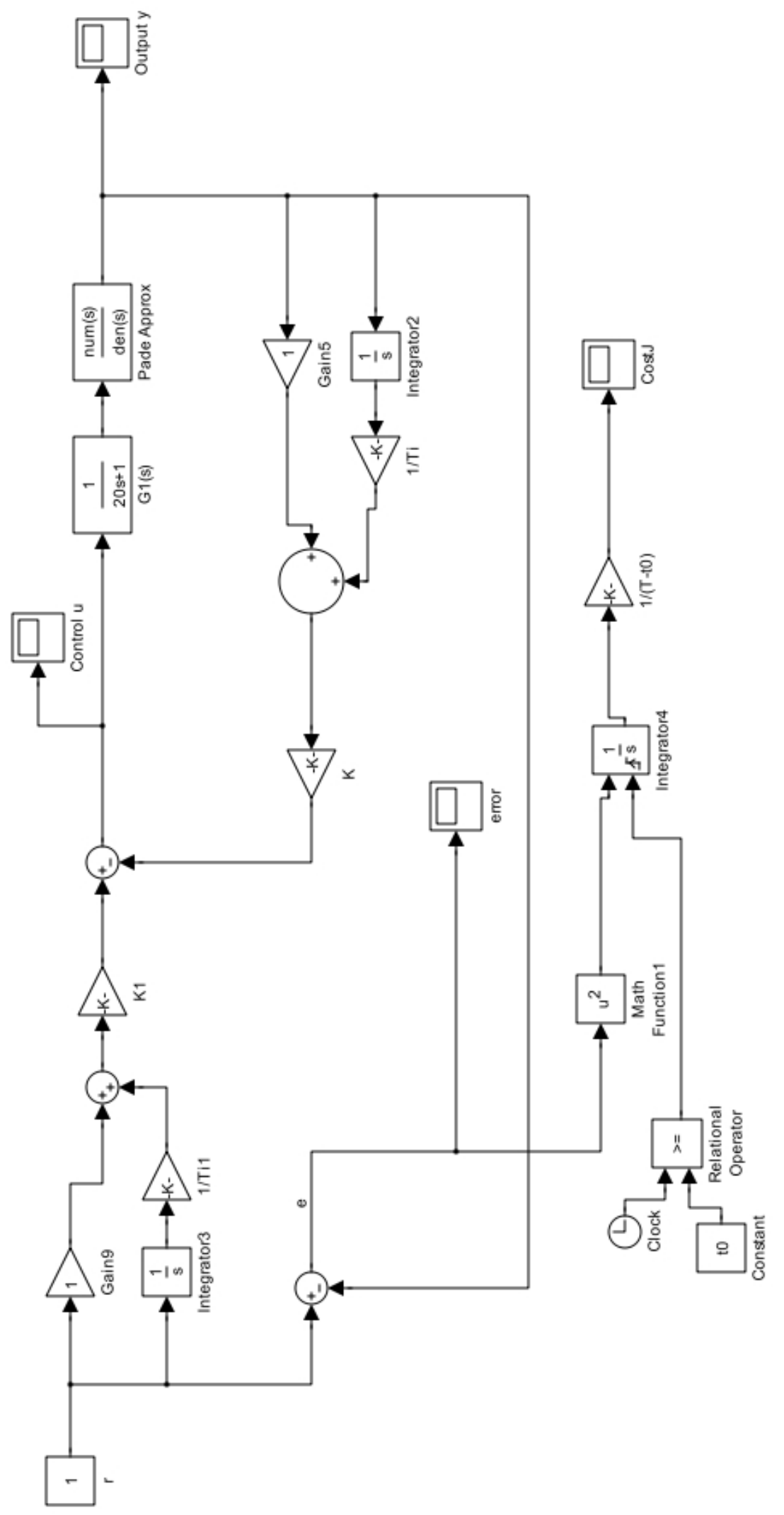

Figure D.1: Simulink model for extremum seeking controller tuning. 


\section{D.4 Optimization Problem A and B}

The following program and Simulink model were used to solve optimization problems A and $\mathrm{B}$ for the simulation examples presented in Chapters 4 to 7.

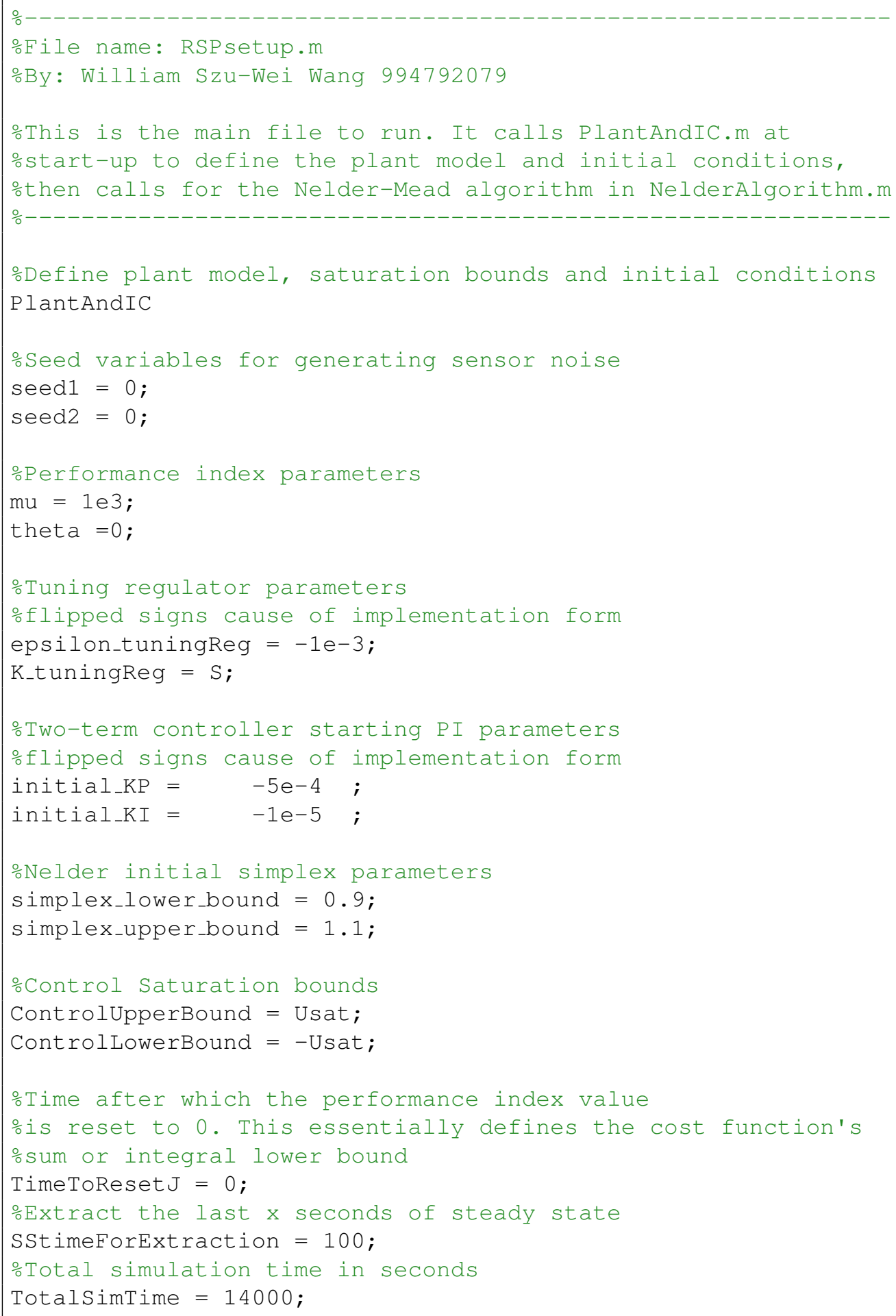




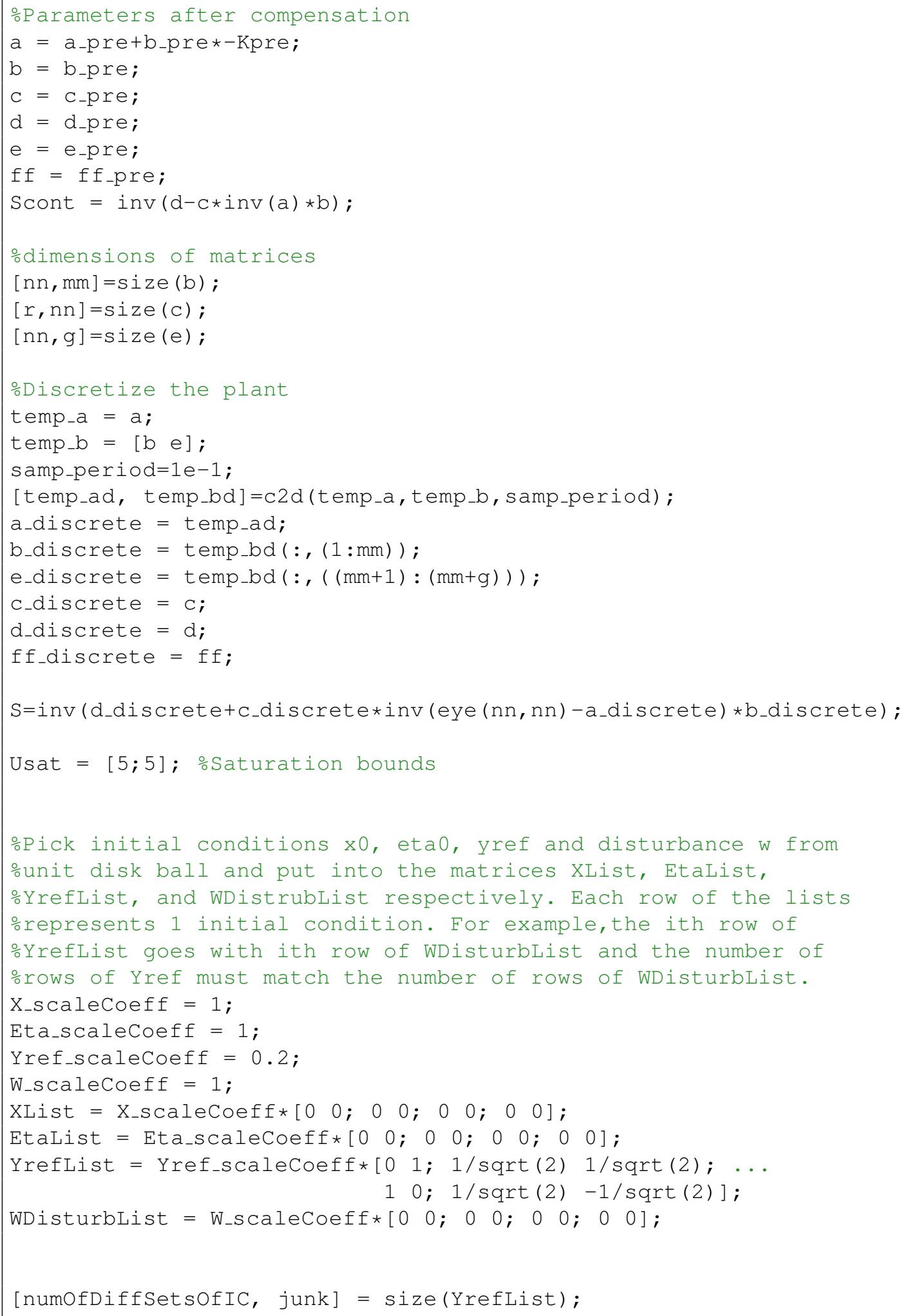




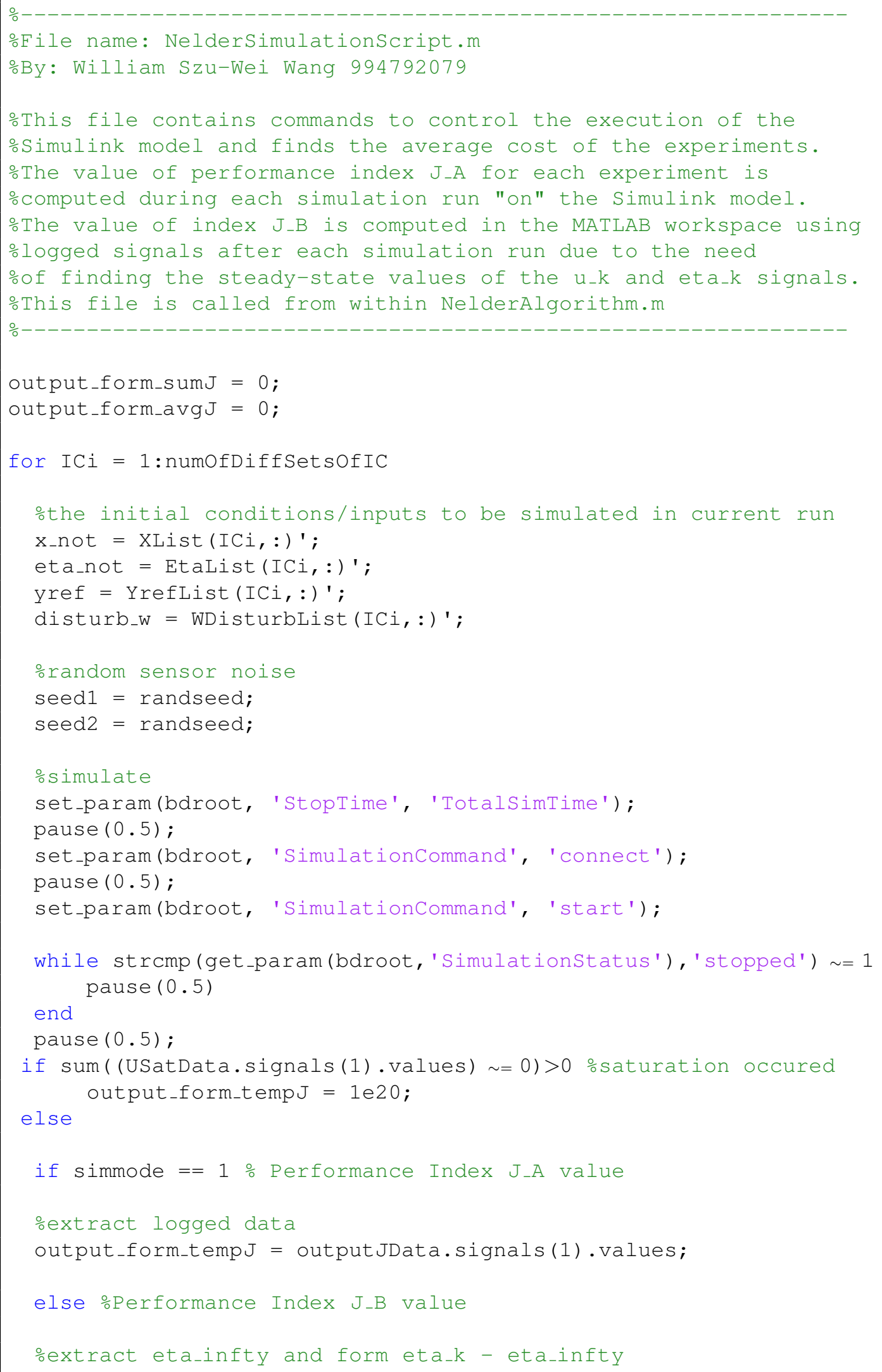




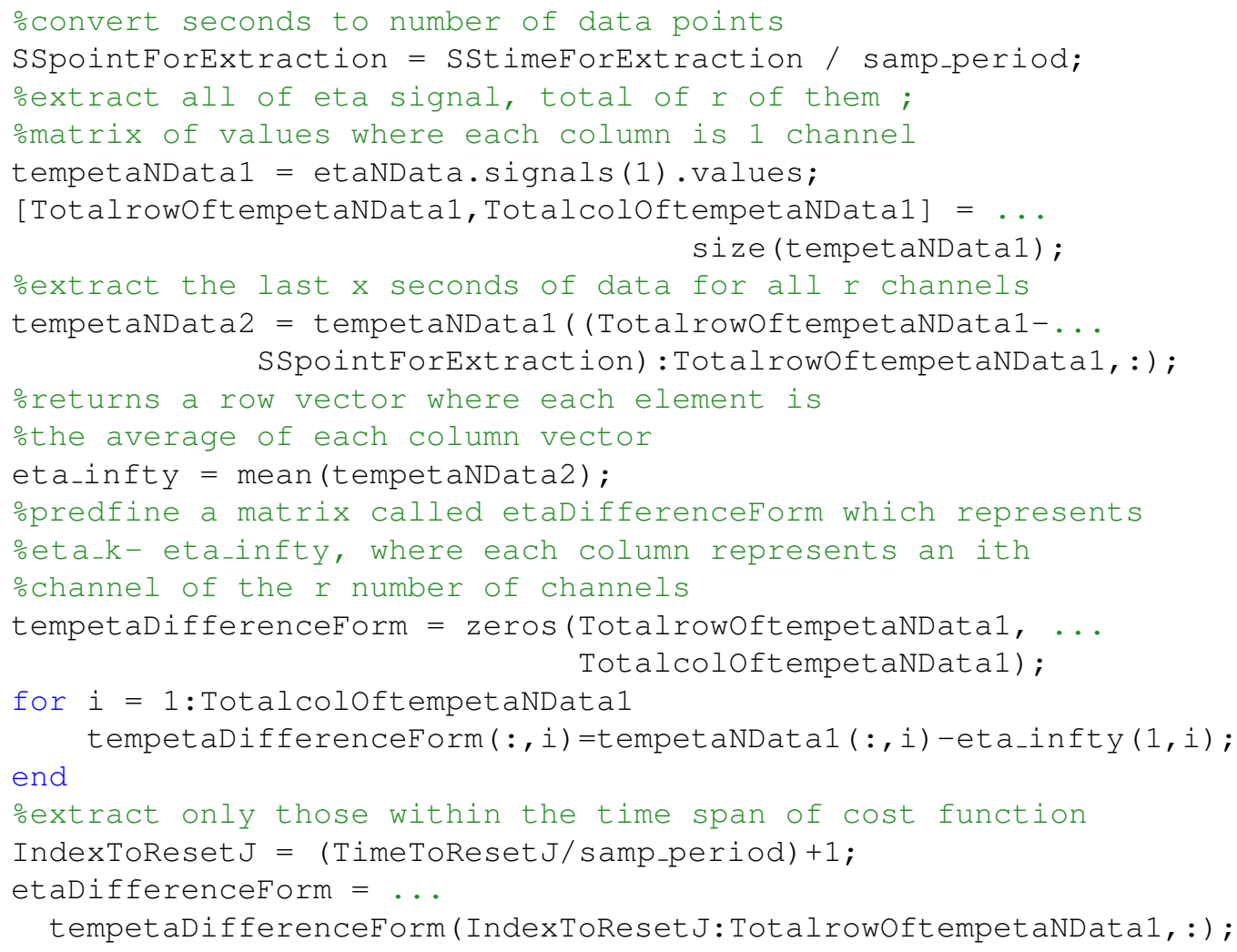




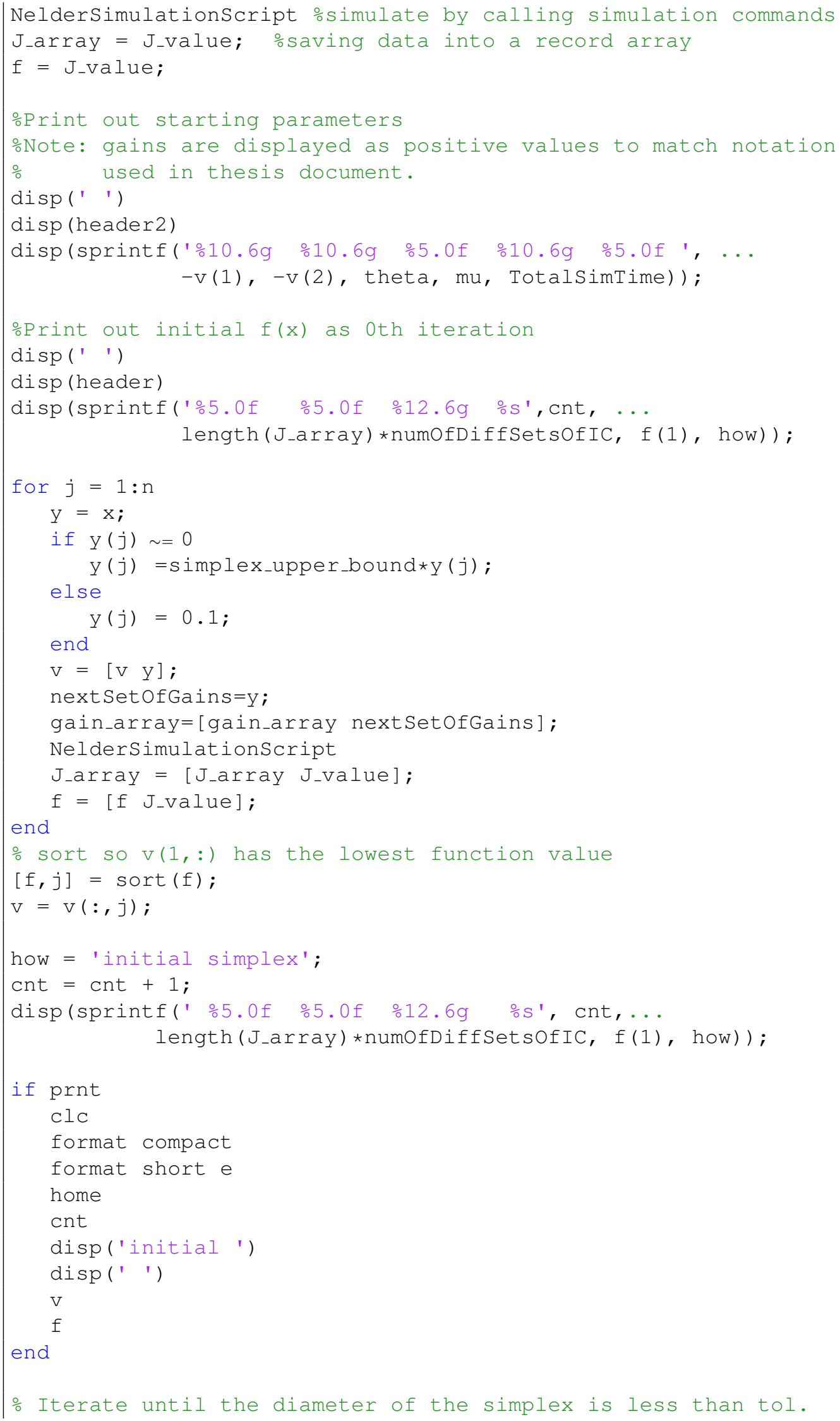




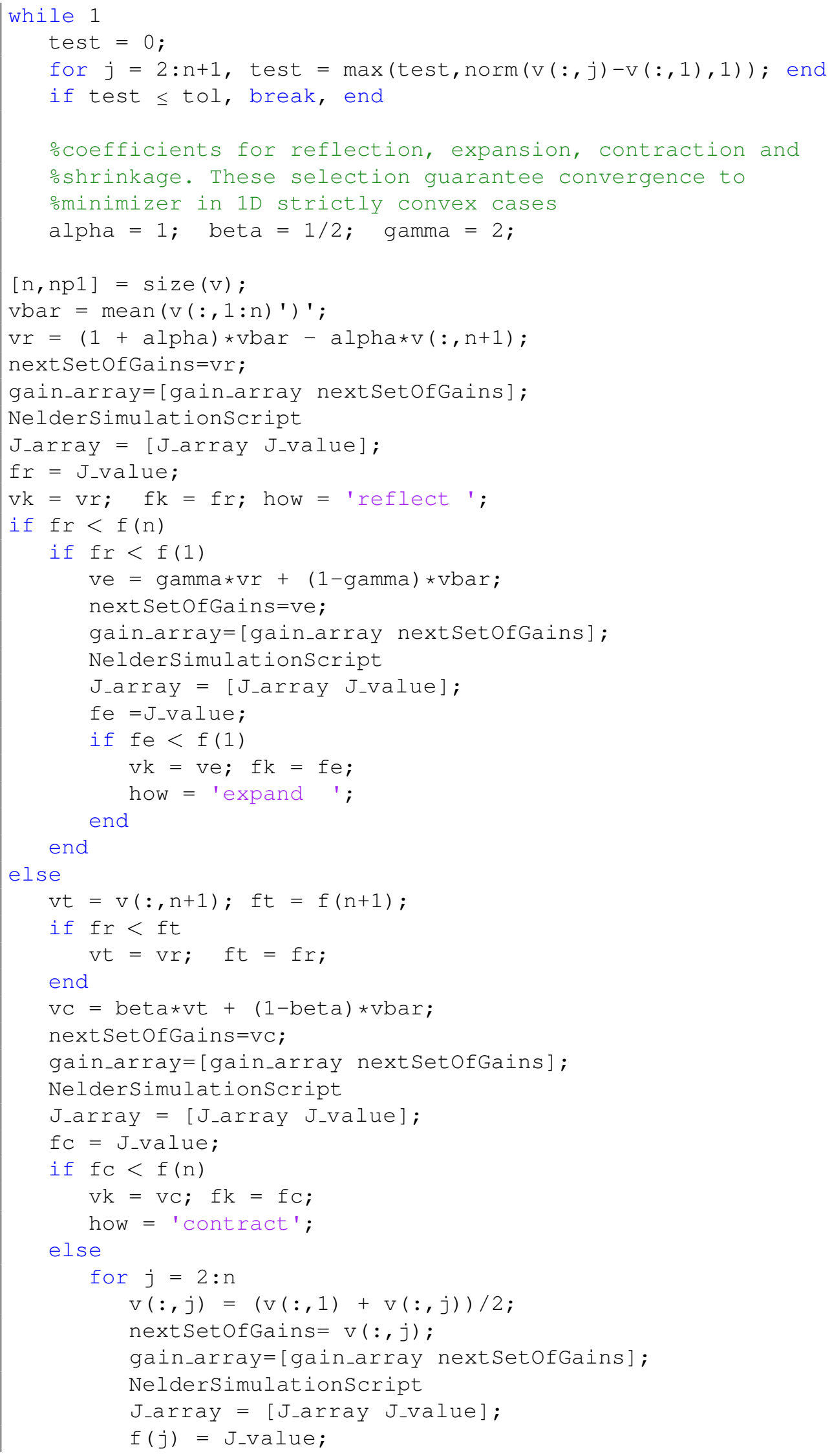




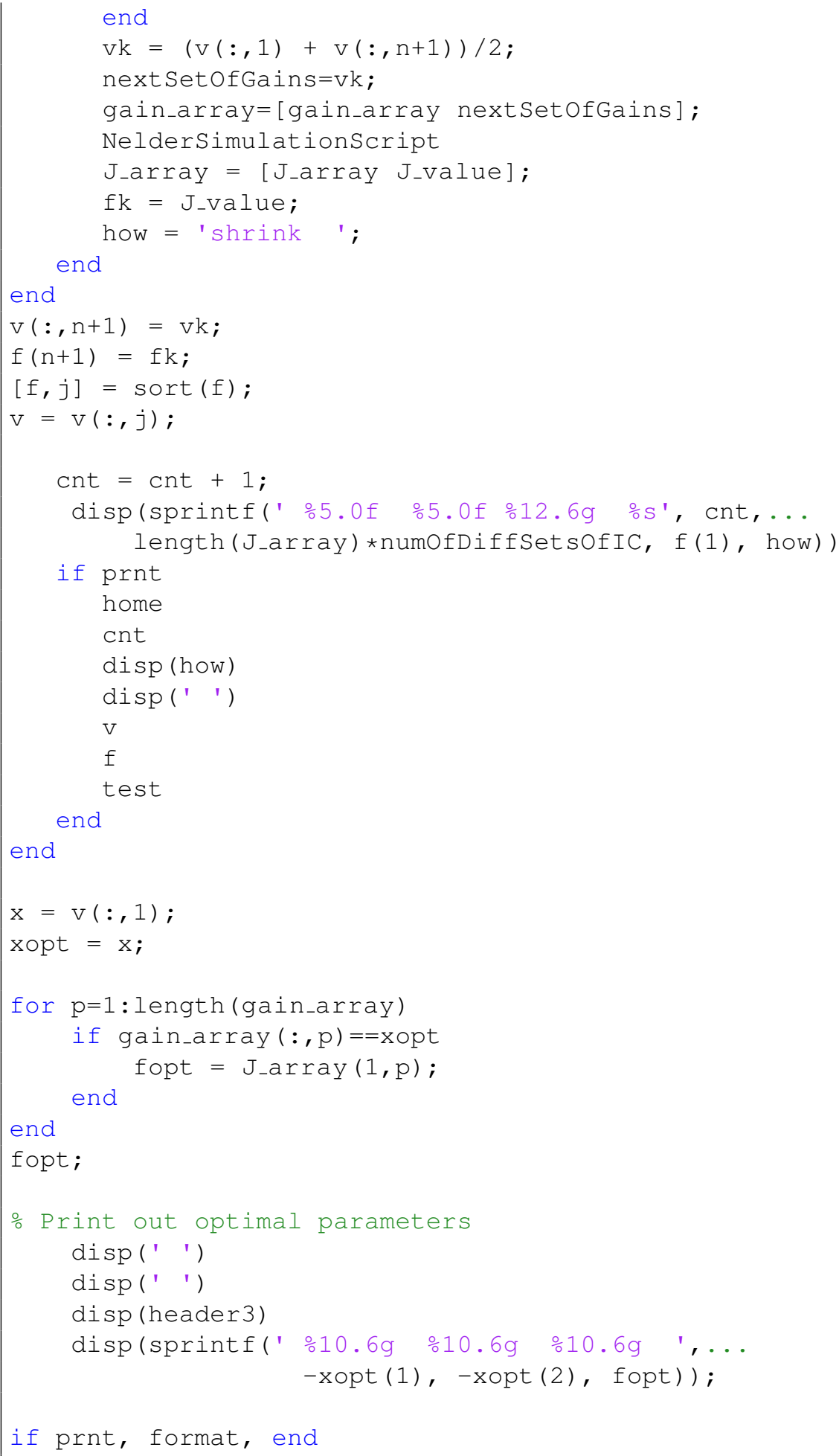




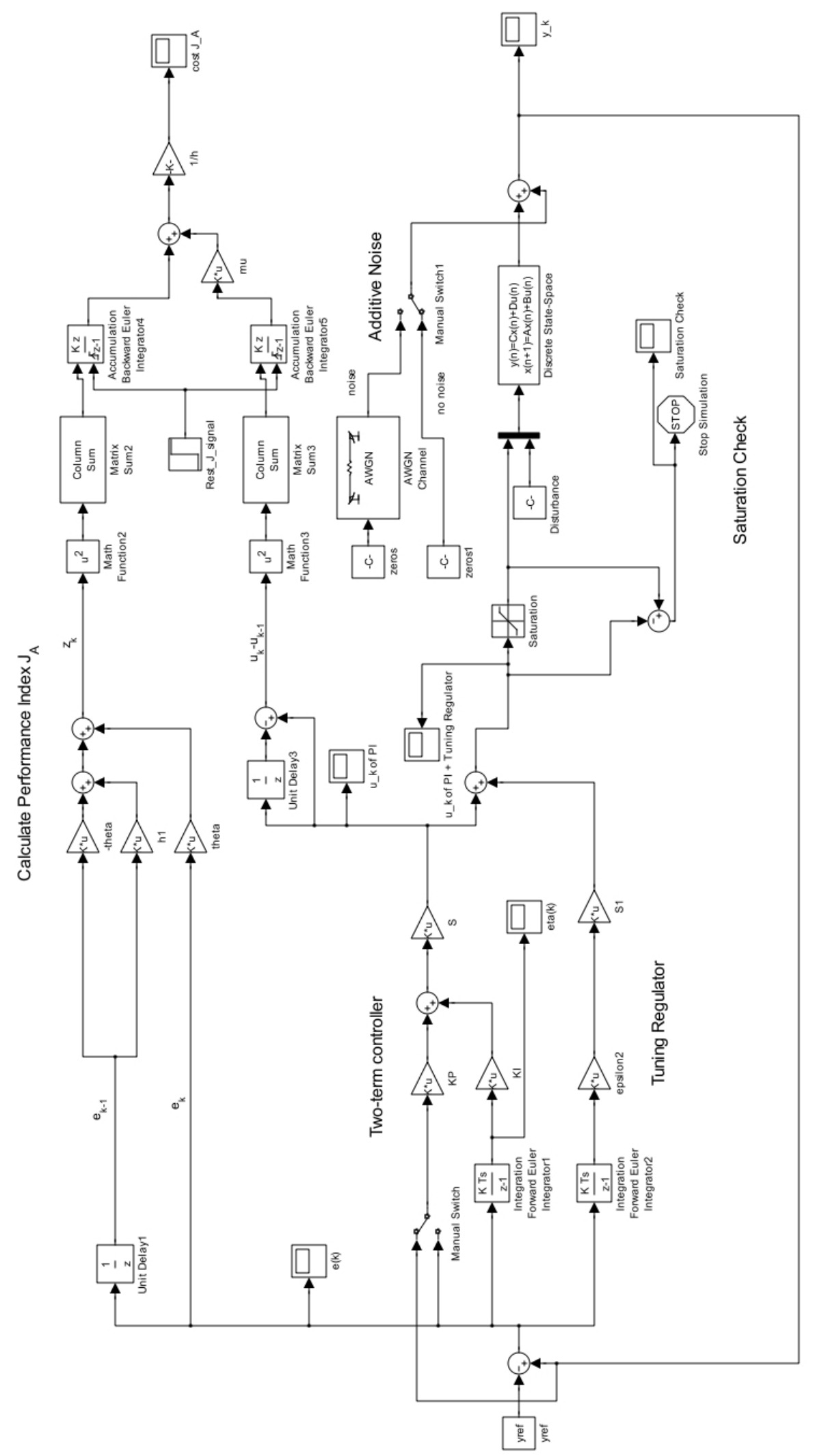

Figure D.2-7: Simulink model for optimization problems A and B. 


\section{D.5 MARTS Experiment}

The following MATLAB files and Simulink model were used to carry out optimization problem $\mathrm{B}$ on the experimental test bed MARTS.

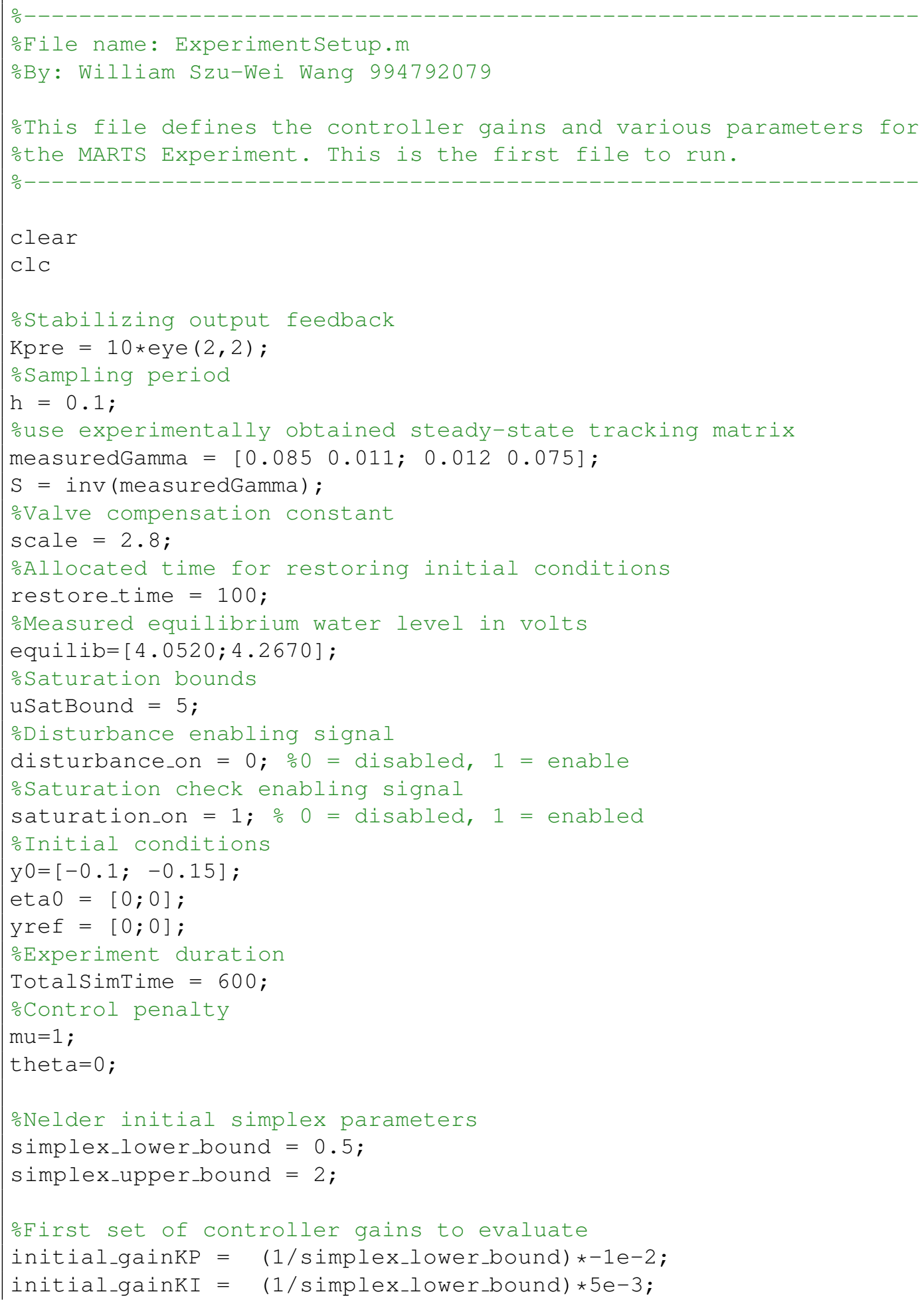




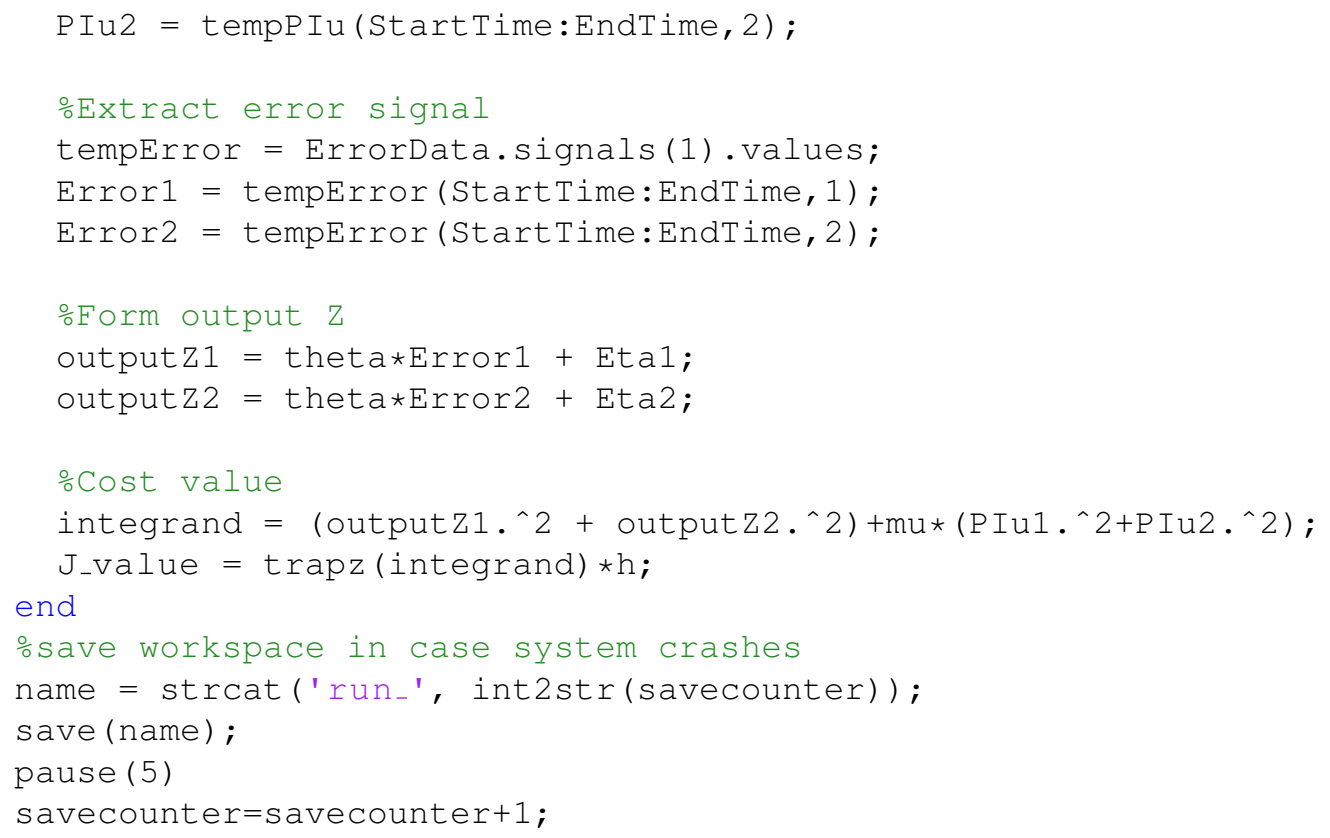




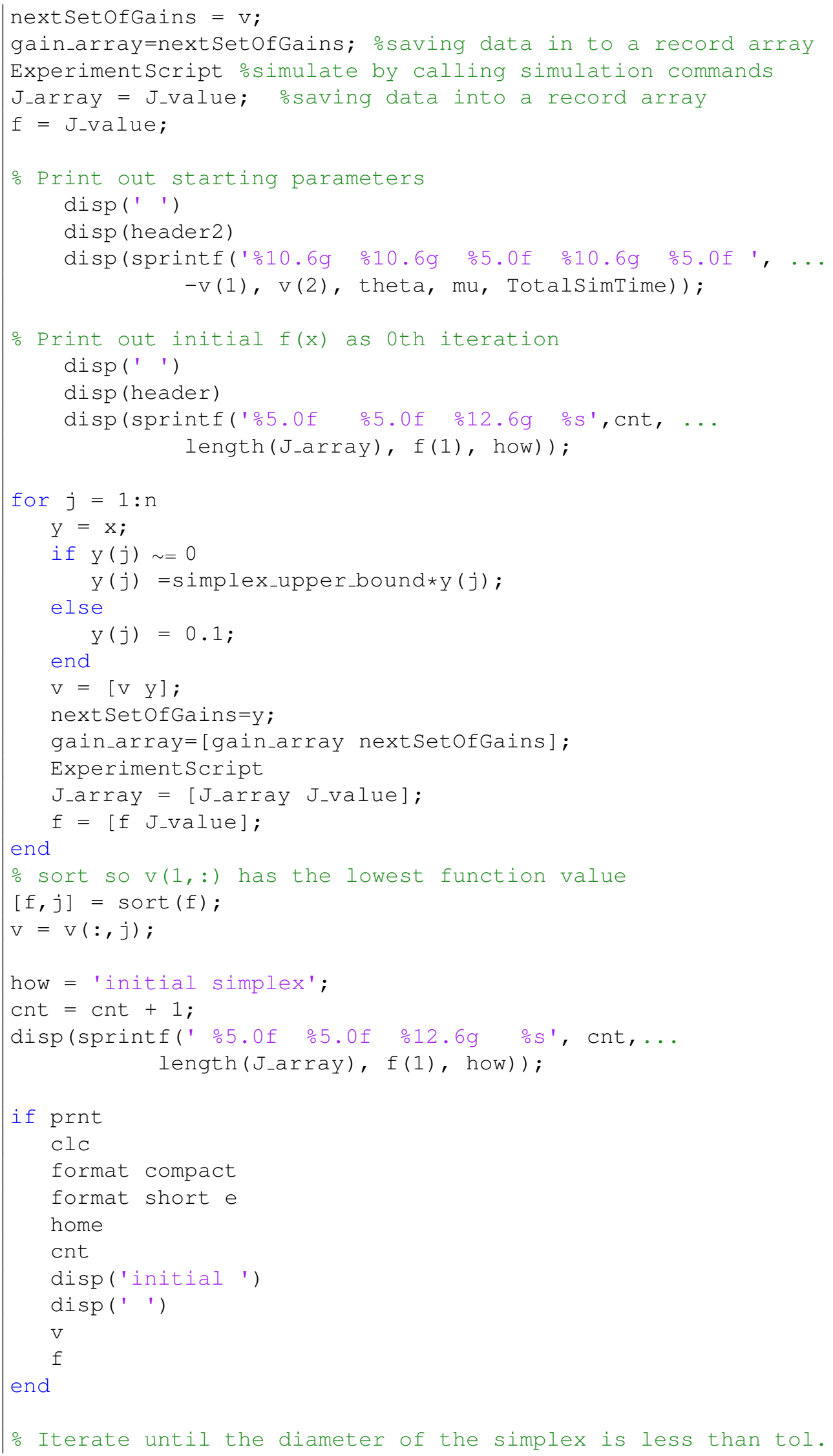




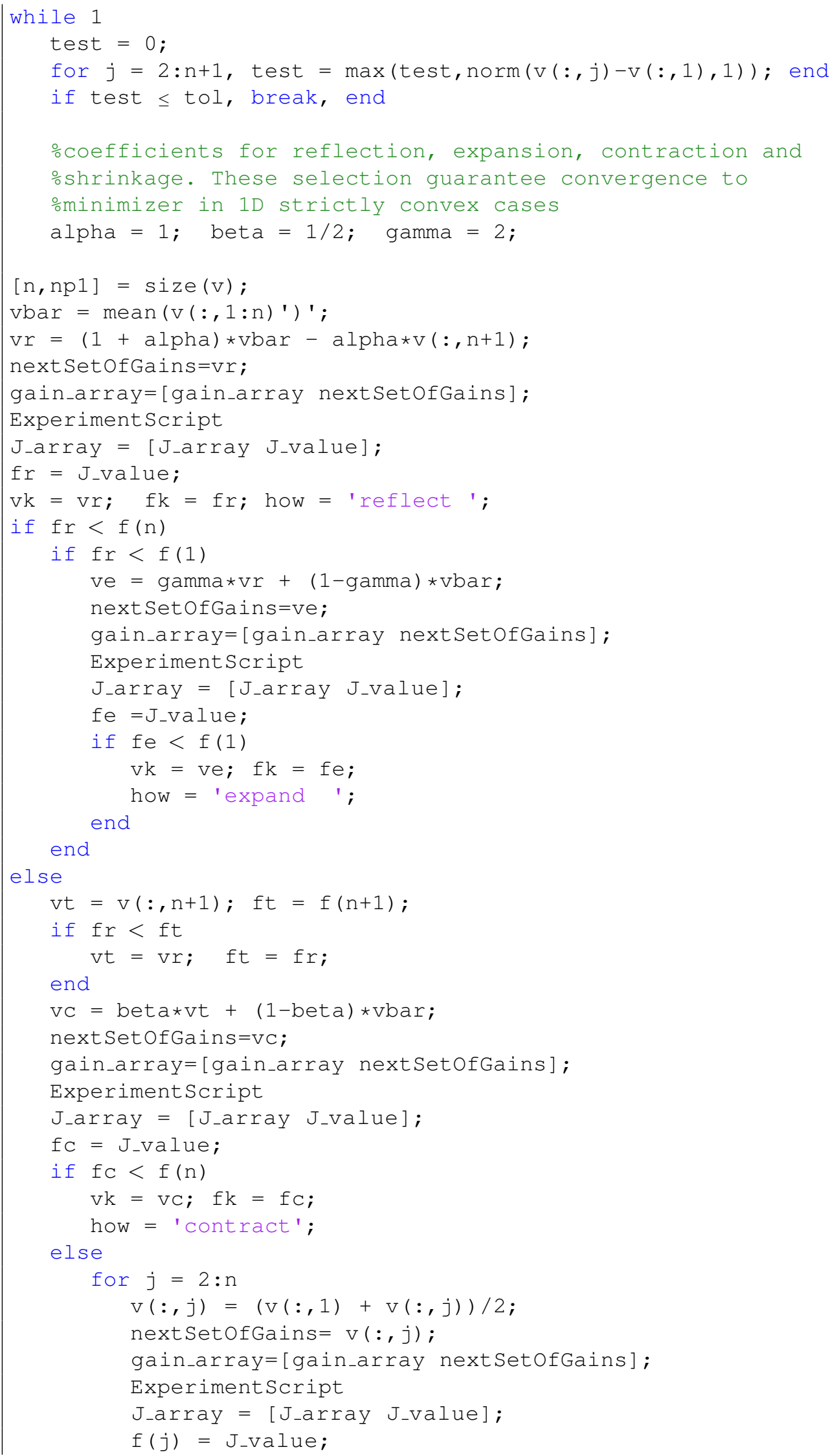




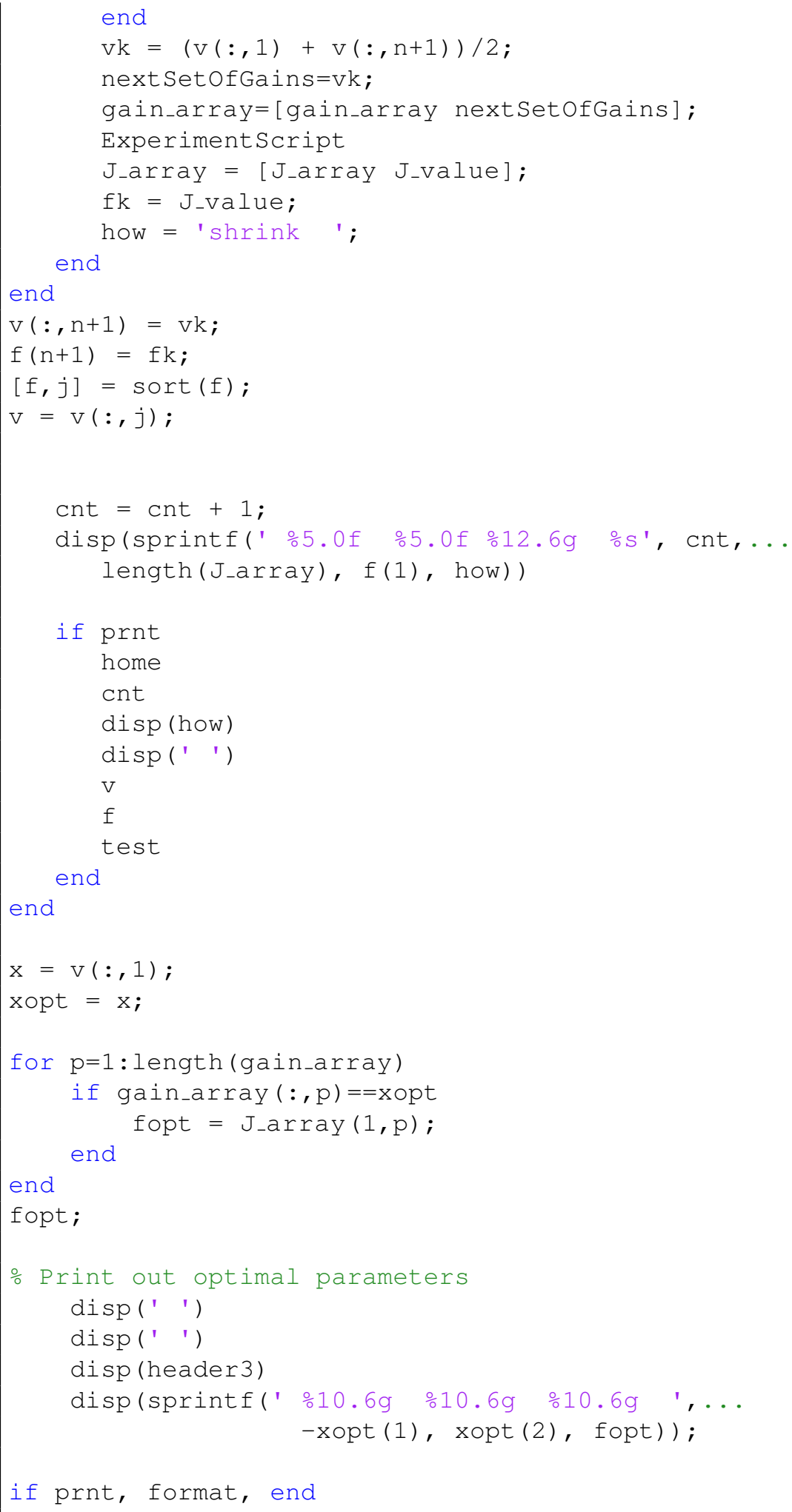




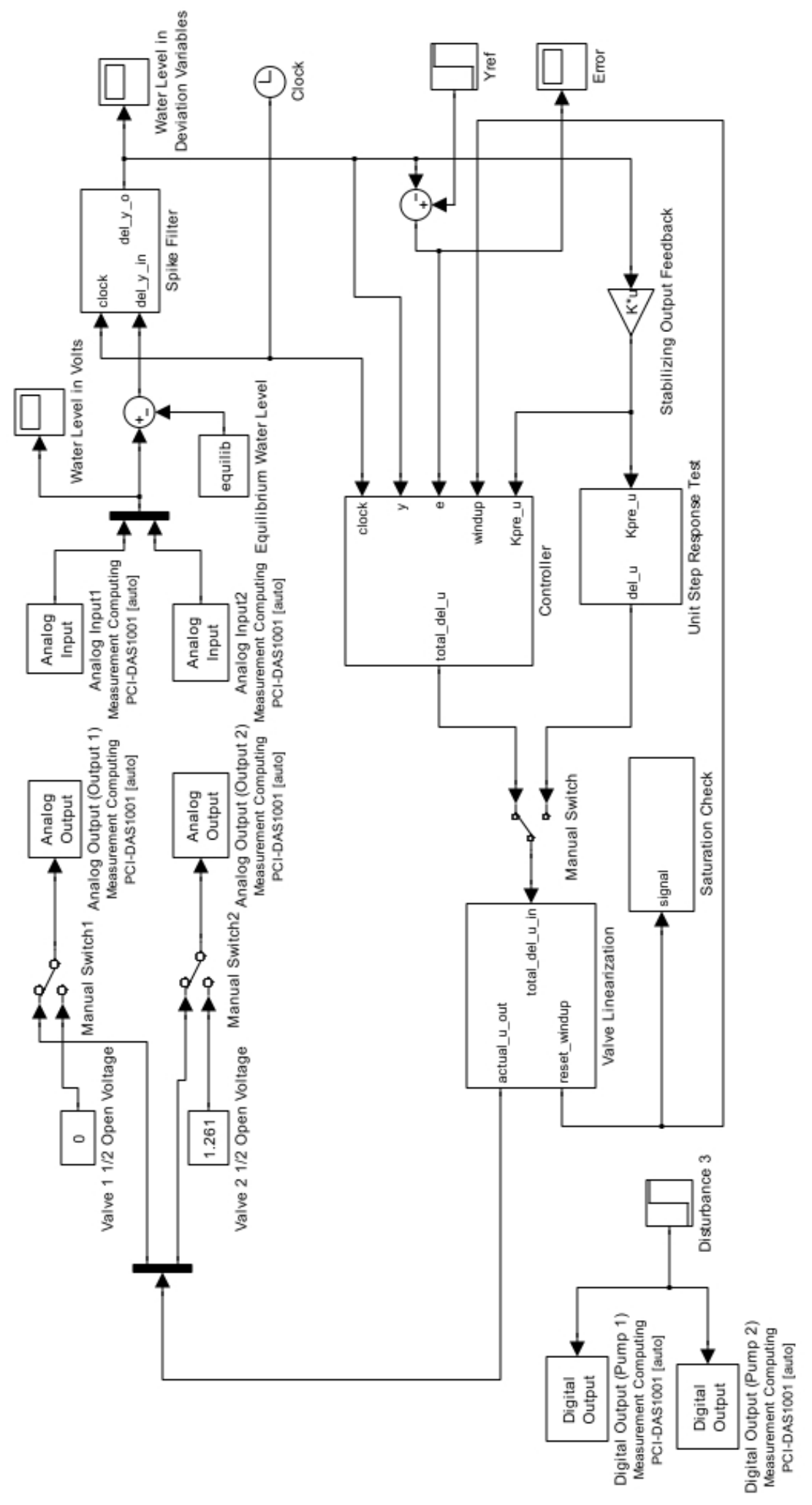

Figure D.3-1: Simulink model for MARTS experiment. 


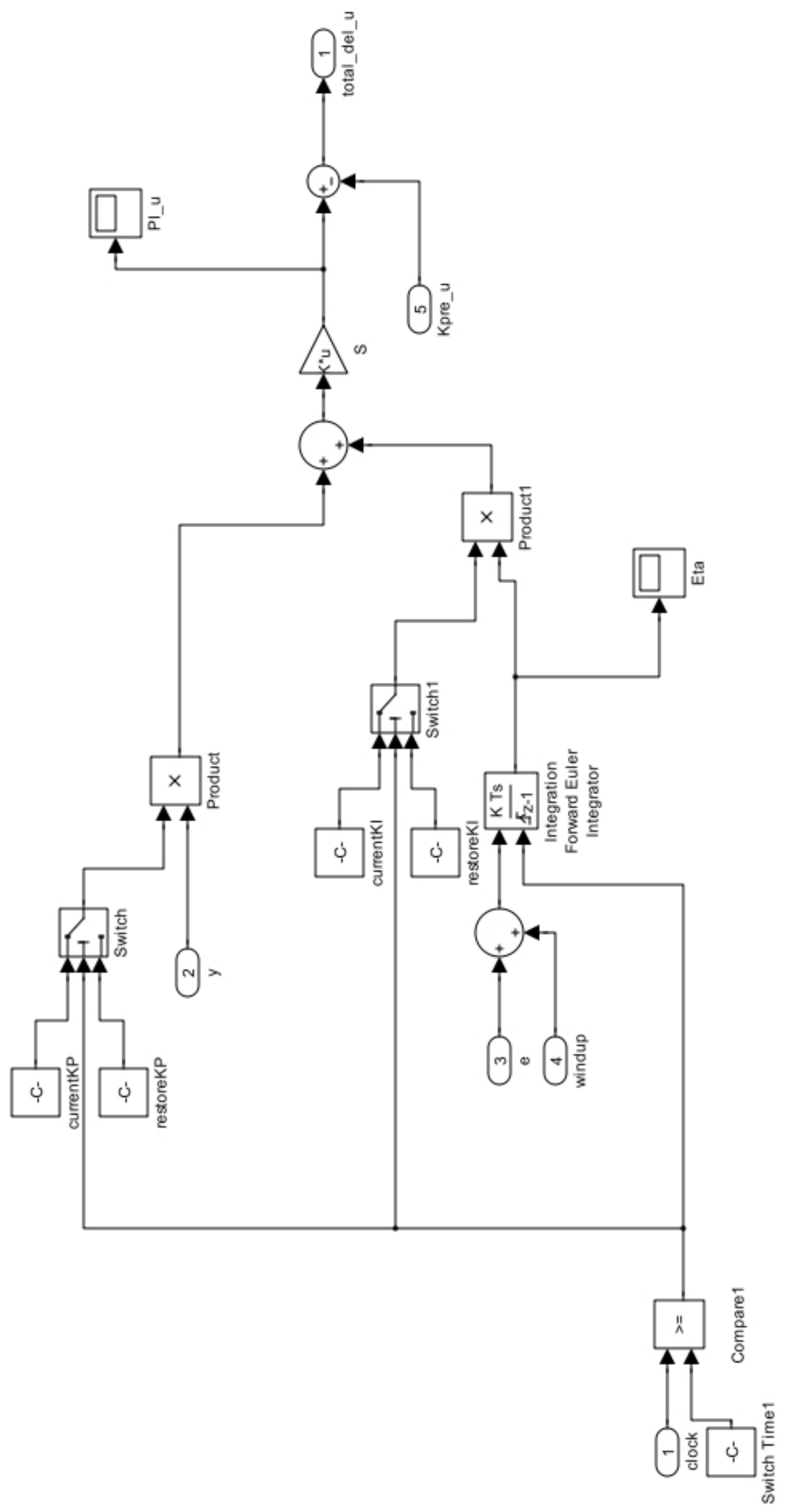

Figure D.3-2: "Controller" subsystem block. 


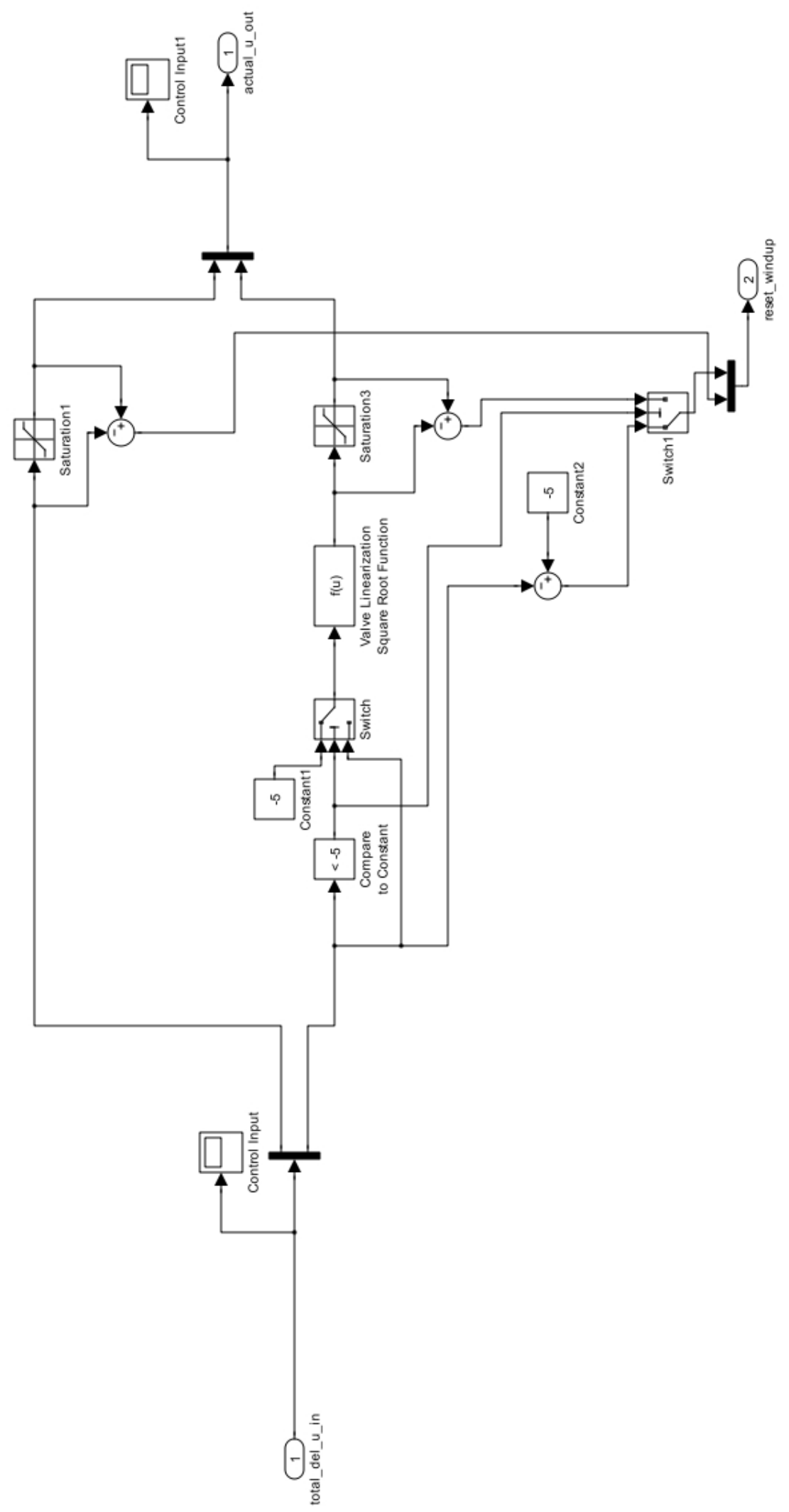

Figure D.3-3: "Valve Linearization" subsystem block. 


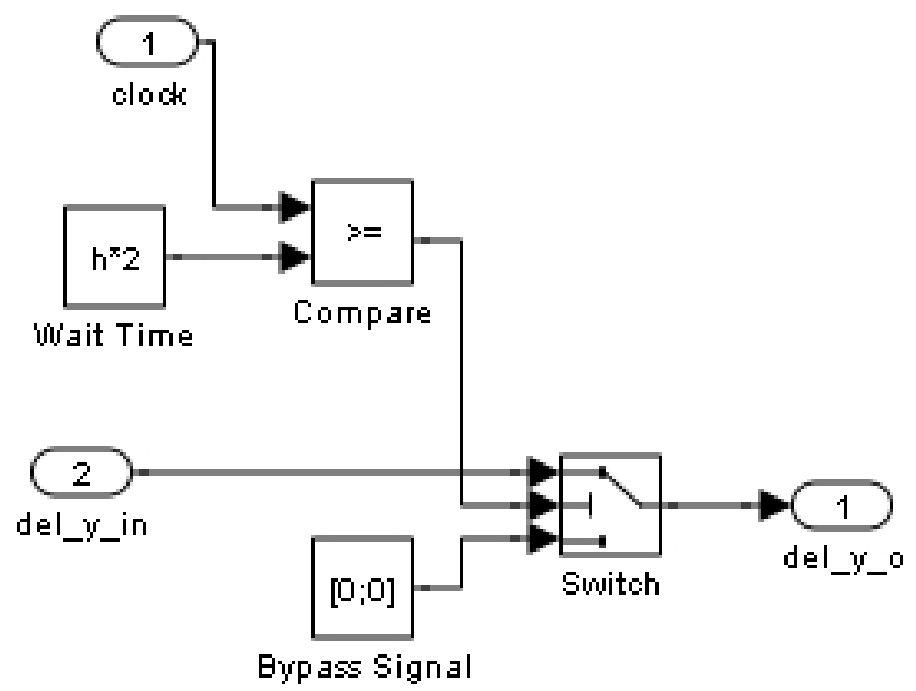

Figure D.3-4: "Spike Filter" subsystem block.

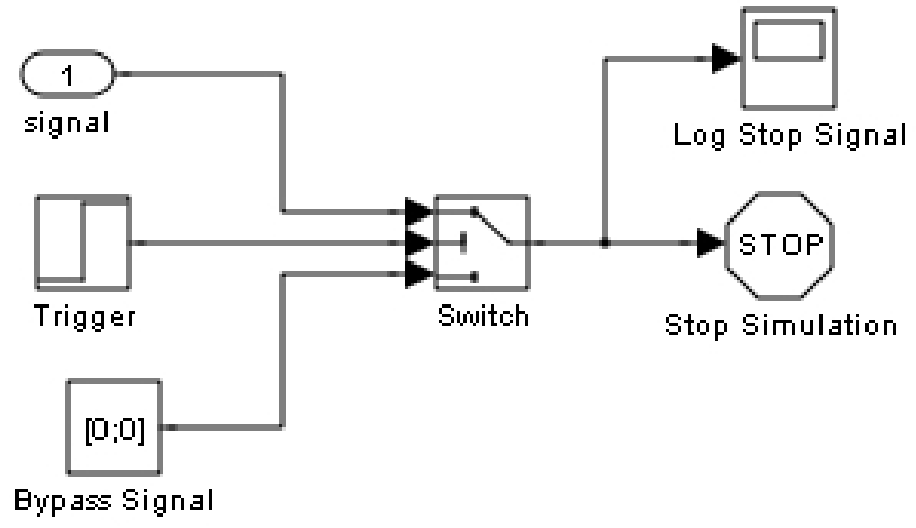

Figure D.3-5: "Saturation check" subsystem block.

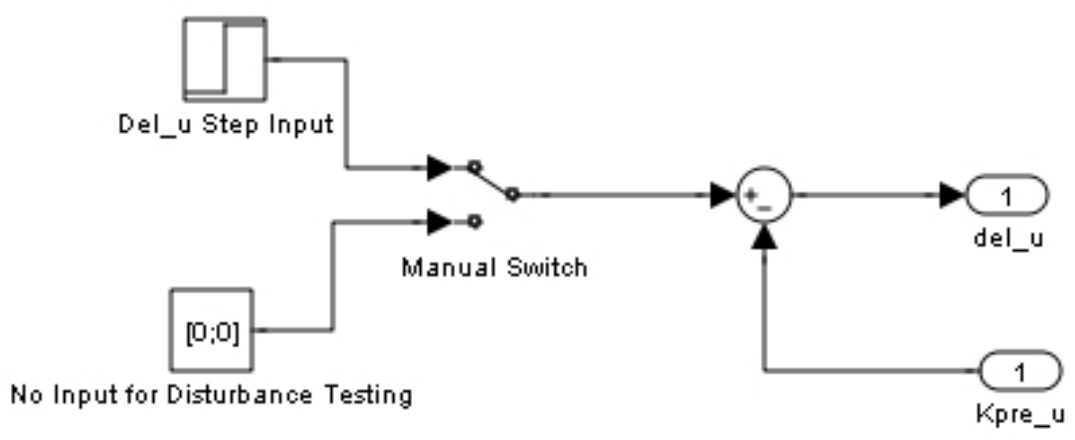

Figure D.3-6: "Unit Step Response Test" subsystem block. 